Prepared for the U.S. Department of Energy

under Contract DE-AC05-76RL01830

\title{
The Smart Grid: An Estimation of the Energy and $\mathrm{CO}_{2}$ Benefits
}

RG Pratt

PJ Balducci

C Gerkensmeyer

S Katipamula

January 2010
MCW Kintner-Meyer

TF Sanquist

KP Schneider

TJ Secrest

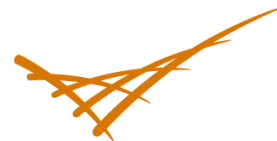

Pacific Northwest

NATIONAL LABORATORY

Proudly Operated by Battelle Since 1965 


\section{DISCLAIMER}

This report was prepared as an account of work sponsored by an agency of the United States Government. Neither the United States Government nor any agency thereof, nor Battelle Memorial Institute, nor any of their employees, makes any warranty, express or implied, or assumes any legal liability or responsibility for the accuracy, completeness, or usefulness of any information, apparatus, product, or process disclosed, or represents that its use would not infringe privately owned rights. Reference herein to any specific commercial product, process, or service by trade name, trademark, manufacturer, or otherwise does not necessarily constitute or imply its endorsement, recommendation, or favoring by the United States Government or any agency thereof, or Battelle Memorial Institute. The views and opinions of authors expressed herein do not necessarily state or reflect those of the United States Government or any agency thereof.

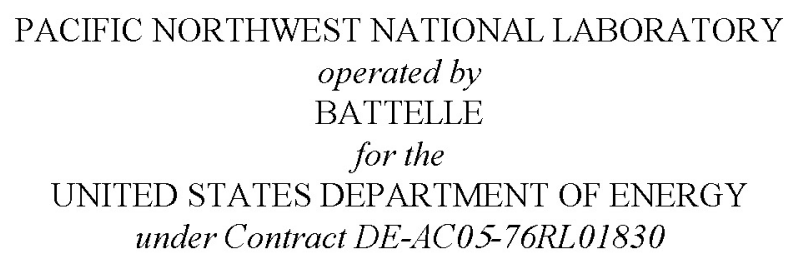

Printed in the United States of America

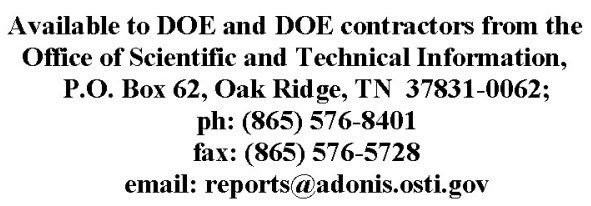

Available to the public from the National Technical Information Service, U.S. Department of Commerce, 5285 Port Royal Rd., Springfield, VA 22161 ph: $(800) 553-6847$ fax: $(703) 605-6900$ email: orders@ntis.fedworld.gov online ordering: http://www.ntis.gov/ordering.htm 


\section{The Smart Grid: An Estimation of the Energy and $\mathrm{CO}_{2}$ Benefits}

RG Pratt

PJ Balducci

C Gerkensmeyer ${ }^{(a)}$

S Katipamula
MCW Kintner-Meyer

TF Sanquist

KP Schneider

TJ Secrest

January 2010

Prepared for

the U.S. Department of Energy

under Contract DE-AC05-76RL01830

Pacific Northwest National Laboratory

Richland, Washington 99352

(a) Benton Rural Electric Association, West Richland, Washington. 



\begin{abstract}
This report articulates nine mechanisms by which the smart grid can reduce energy use and carbon impacts associated with electricity generation and delivery. The quantitative estimates of potential reductions in electricity sector energy and associated $\mathrm{CO}_{2}$ emissions presented are based on a survey of published results and simple analyses. This report does not attempt to justify the cost effectiveness of the smart grid, which to date has been based primarily upon the twin pillars of cost-effective operation and improved reliability. Rather, it attempts to quantify the additional energy and $\mathrm{CO}_{2}$ emission benefits inherent in the smart grid's potential contribution to the nation's goal of mitigating climate change by reducing the carbon footprint of the electric power system.
\end{abstract}





\section{Summary}

This report provides an assessment of nine mechanisms by which the smart grid can reduce energy use and carbon impacts associated with electricity generation and delivery. To the extent possible, the associated reductions in electricity and $\mathrm{CO}_{2}$ emissions were quantified to illustrate the benefits inherent in the smart grid's potential contribution to the nation's goal of mitigating climate change from reducing the carbon footprint of the electric power system. Environmental impacts to air and water quality and land use were not considered, nor were impacts on end users that rely upon natural gas as their energy source.

The reductions in electric utility electricity and $\mathrm{CO}_{2}$ emissions in 2030 attributable to the nine mechanisms by direct and indirect effect are shown in Table S.1. The direct reductions were calculated for the mechanisms that affected electricity and $\mathrm{CO}_{2}$ emissions directly through implementation of the smart grid technologies. Indirect reductions are derived by translating the estimated cost savings in energy and/or capacity into their energy and carbon equivalents through purchase of additional costeffective energy efficiency. This can represent a policy decision to reinvest the savings to purchase additional more cost effective energy efficiency and renewable resources.

Table S.1. Potential Reductions in Electricity and $\mathrm{CO}_{2}$ Emissions in 2030 Attributable to Smart Grid Technologies

\begin{tabular}{lcc}
\hline \multicolumn{1}{c}{ Mechanism } & \multicolumn{2}{c}{$\begin{array}{c}\text { Reductions in Electricity Sector } \\
\text { Energy and } \mathrm{CO}_{2} \text { Emissions }^{(\mathrm{a})}\end{array}$} \\
\cline { 2 - 3 } & Direct (\%) & Indirect (\%) \\
\hline Conservation Effect of Consumer Information and Feedback Systems & 3 & - \\
Joint Marketing of Energy Efficiency and Demand Response Programs & - & 0 \\
Deployment of Diagnostics in Residential and Small/Medium Commercial & 3 & - \\
Buildings & & 0.5 \\
Measurement \& Verification (M\&V) for Energy Efficiency Programs & 1 & - \\
Shifting Load to More Efficient Generation & $<0.1$ & - \\
Support Additional Electric Vehicles and Plug-In Hybrid Electric Vehicles & 3 & - \\
Conservation Voltage Reduction and Advanced Voltage Control & 2 & 5 \\
Support Penetration of Renewable Wind and Solar Generation & $<0.1$ & $\mathbf{6}$ \\
(25\% renewable portfolio standard [RPS]) & & $\mathbf{1 2}$ \\
Total Reduction & & \\
\hline
\end{tabular}

(a) Assumes $100 \%$ penetration of the smart grid technologies.

The estimates in Table S.1 are based on the annual electricity supplied to the U.S. grid and the associated $\mathrm{CO}_{2}$ emissions in 2030, as forecast by the U.S. Energy Information Agency. They represent the percentage reduction in the annual U.S. electrical energy production and resulting $\mathrm{CO}_{2}$ reductions, based on the emissions of average U.S. generating power plant. This allows the percentage reductions to be placed in context with RPSs for their electric system that have been already adopted by many states, typically $20 \%$ or more over a period of one or two decades. 
The uncertainties in these estimates are relatively high, based on the range of estimates provided by the studies drawn upon for this report, and the judgment of the authors. While the individual reduction estimates are typically judged to be uncertain in a range of $\pm 50 \%$, and in some cases larger, the variety inherent in the mechanisms suggests a higher level of confidence when their combined effect is considered.

The estimates assume full deployment (100\% penetration) of smart grid technologies. Since the reductions are expected to be linear with respect to penetration level, this assumption enables the estimates to be readily scaled to lower levels of assumed penetration.

The importance of these reduction estimates is in their combined effect. While several of the mechanisms are estimated to have small or negligible impacts, five of the mechanisms could potentially provide reductions of over $1 \%$. Moreover, the combined effect of the direct mechanisms is $12 \%$, and the indirect mechanisms total $6 \%$ of energy and emissions for the U.S. electricity sector. These correspond to $5 \%$ and $2 \%$ of the U.S. total energy consumption and energy-related $\mathrm{CO}_{2}$ emissions for all sectors (including electricity). The magnitude of these reductions suggests that, while a smart grid is not the primary mechanism for achieving aggressive national goals for energy and carbon savings, it is capable of providing a very substantial contribution to the goals for the electricity sector. Further, a smart grid may help overcome barriers to deployment of distributed solar renewables at penetrations higher than $20 \%$.

Recommendations and issues specific to each of the mechanisms are provided in the report to guide research and implementation efforts. Formulation of a common set of recommendations and issues is difficult due to the diversity and specificity of the mechanisms. Having said this, general

recommendations for further examination are:

- First and foremost, all technical mechanisms need to be considered in greater analytical depth to more rigorously address the quantification of and uncertainties for the estimated reductions in electricity and $\mathrm{CO}_{2}$ emissions to help set priorities for development of smart grid technologies.

- Customer feedback is necessary for the effective implementation and communication of energy efficiency and demand response management programs to maintain sustained levels of reduction. Central to effective feedback is to understand and reduce the uncertainty associated with consumer behavior and response in order to design effective feedback mechanisms.

- Coupled with feedback, the effectiveness of customer-side programs can be increased by leveraging smart grid assets to provide long-term $\mathrm{M} \& \mathrm{~V}$ and diagnostics at little additional cost for the required analysis capabilities. The focus of this need is for analytic methods and software technologies, with decisions to locate the capability centrally or on the customer's side of the meter.

- Also evidenced in Table S.1, key research needs are in the areas of how smart grid technologies can support the 1) integration of renewable resources above the $20 \%$ RPS through a combination of demand response, renewable resources, and storage technologies, 2) addition of increased levels of electric vehicles to best utilize generating assets, and 3) management of voltage control and losses within the transmission and distribution system to reduce losses and increase reliability.

A key issue that will impact the penetration of the smart grid technology, at least components of the technology that bear upon its functionality, is the acceptance by federal and state regulatory bodies. A major driver for this acceptance is the extent to which the smart grid technology proves to be a costeffective replacement for traditional grid infrastructure while providing equal or improved levels of power 
quality and reliability. This highlights the need for a quantitative method to define and monetize improvements in power reliability and quality that would be enabled by smart grid technologies. In conjunction, is the need to involve stakeholders in adapting the business and regulatory models (planning, monetary, risk, incentives, etc.) from a centralized power system to a more decentralized system.

A second issue is that to realize the estimated reductions the smart grid can deliver, offsetting increases in consumption are expected from servers located in every distribution substation and demand response/GFA devices installed in the stock of appliances. The combined effect of the two offsets may increase the electric utility sector energy and emission reductions by approximately $0.1 \%$ to $0.4 \%$. While the magnitude of this increase is small and may not be considered important, it does point to the need for technology developers to minimize the increased loads of smart grid technologies. 



\section{Acknowledgments}

The authors would like to acknowledge the input from DOE, EPA with particular thanks to Stacy Angel, James Critchfield, Zoltan Jung, and Priya Sreedharan (AAAS Fellow) of U.S. EPA for their detailed review and constructive suggestions. The authors assume responsibility for any errors and omissions. 



\section{Acronyms and Abbreviations}

\begin{tabular}{|c|c|}
\hline $\mathrm{AC}$ & alternating current \\
\hline AEO & Annual Energy Outlook \\
\hline AFDD & automated fault detection and diagnostics \\
\hline AMI & advanced metering infrastructure \\
\hline ANSI & American National Standards Institute \\
\hline BPA & Bonneville Power Administration \\
\hline Btu & British thermal unit(s) \\
\hline $\mathrm{CFC}$ & chlorofluorocarbon \\
\hline $\mathrm{CT}$ & combustion turbine \\
\hline CVR & conservation voltage reduction \\
\hline CVRf & conservation voltage reduction savings factor \\
\hline $\mathrm{DC}$ & direct current \\
\hline DEGI & dispatchable emergency generator initiatives \\
\hline DOE & U.S. Department of Energy \\
\hline EAC & Electricity Advisory Committee \\
\hline ECAR & East Central Area Reliability Coordinating Agreement \\
\hline EEI & Edison Electric Institute \\
\hline EIA & U.S. Energy Information Administration \\
\hline EISA & Energy Independence and Security Act \\
\hline EMCS & energy management and control systems \\
\hline EPA & U.S. Environmental Protection Agency \\
\hline EPRI & Electric Power Research Institute \\
\hline ERCOT & Electric Reliability Council of Texas \\
\hline ESPC & Energy Savings Performance Contract \\
\hline ESPP & Energy-Smart Pricing Plan \\
\hline EV & electric vehicle \\
\hline FEMP & Federal Energy Management Program \\
\hline FERC & Federal Energy Regulatory Commission \\
\hline GFA & grid friendly appliance \\
\hline GHG & greenhouse gas \\
\hline GREET & greenhouse gases, regulated emissions, and energy use in transportation \\
\hline GW & gigawatt, one billion watts of generating capacity \\
\hline $\mathrm{HCFC}$ & hydro-chlorofluorocarbon \\
\hline HEV & hybrid electric vehicle \\
\hline HVAC & heating, ventilating, and air conditioning \\
\hline
\end{tabular}


ICT

IM

IPMVP

ISO

$\mathrm{kW}$

$\mathrm{kWh}$

$\mathrm{LC} / \mathrm{S}$

LDV

LoanSTAR

$\mathrm{m}$

$\mathrm{m}^{2}$

MMT

$\mathrm{M} \& \mathrm{~V}$

MW

MWh

NERC

NHTS

NYSERDA

OE

ORNL

PDRE

PHEV

PLRP

PNNL

PRISM

PV

RECAP

RPS

RTO

SCADA

SUV

T\&D

TWh

VAR

VMT information and control technologies

interval meters

International Performance Measurement \& Verification Protocol

Independent System Operations

kilowatt(s)

kilowatt hour(s)

load curtailment/shifting

light-duty vehicle

Loans to Save Taxes and Resources

meter(s)

square meter(s)

million metric tonnes

measurement $\&$ verification

megawatt(s)

megawatt hour(s)

North American Electric Reliability Corporation

National Household Travel Survey

New York State Energy Research and Development Authority

Office of Electricity Delivery and Energy Reliability

Oak Ridge National Laboratory

permanent demand reduction efforts

plug-in hybrid electric vehicle

Peak Load Reduction Program

Pacific Northwest National Laboratory

Princeton Scorekeeping Method

photovoltaic

Regional Capacity Planning

renewable portfolio standard

Regional Transmission Organization

supervisory control and data acquisition

Sport Utility Vehicle

transmission and distribution

terawatt hour(s)

volt-ampere reactive

vehicle-mile(s) traveled 


\section{Contents}

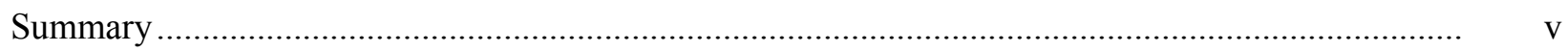

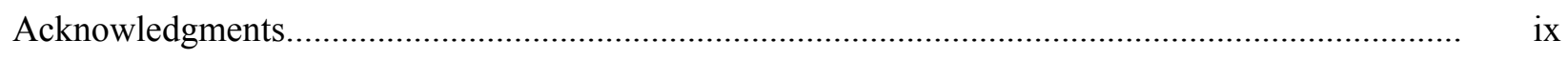

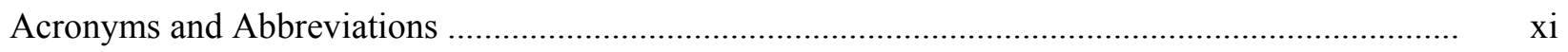

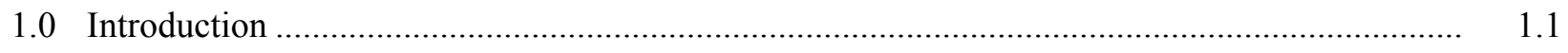

2.0 Smart Grid - What it Is, What it Does, and Who it Benefits............................................... 2.1

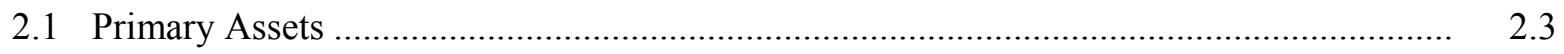

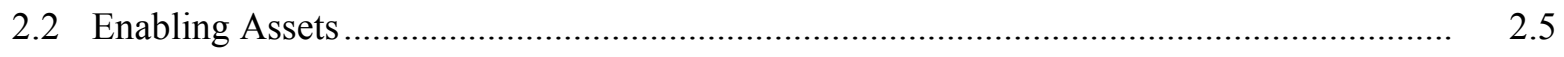

2.3 Functions: Operational Objectives .......................................................................... 2.5

2.4 The Business Case for a Smart Grid ........................................................................... 2.7

3.0 Mechanism Methodology and Summaries ..................................................................... 3.1

3.1 The Smart Grid and Energy Efficiency ....................................................................... 3.5

3.1.1 Conservation Effect of Consumer Information and Feedback Systems ................... 3.7

3.1.2 Joint Marketing of Energy Efficiency and Demand Response Programs ............... 3.10

3.1.3 Key Enabling Technology: Disaggregation of Total Loads into End Uses ............. 3.11

3.1.4 Deployment of Diagnostics in Residential and Small/Medium Commercial Buildings ................................................................................................ $\quad 3.17$

3.1.5 Measurement \& Verification for Energy Efficiency Programs ............................... 3.19

3.1.6 Shifting Load to More Efficient Generation ........................................................... 3.22

3.1.7 Support Additional Electric Vehicles and Plug-In Hybrid Electric Vehicles ........... 3.24

3.1.8 Conservation Voltage Reduction and Advanced Voltage Control ........................... 3.27

3.2 The Smart Grid and Renewables................................................................................. 3.29

3.2.1 Support Penetration of Renewable Solar Generation .......................................... 3.32

3.2.2 Support Penetration of Renewable Wind Generation ......................................... 3.34

4.0 Comparison with Related Studies..................................................................................

4.1 Review of Related Studies ..................................................................................... 4.1

4.1.1 Electric Power Research Institute Green Grid Study .......................................... 4.1

4.1.2 Climate Group/Information and Control Technologies Report............................... 4.2

4.1.3 Hledik Article: How Green is the Smart Grid? ................................................... 4.4

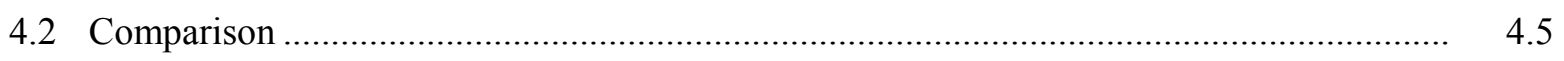

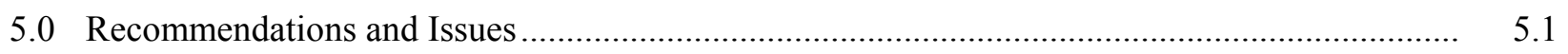

5.1 Mechanism Recommendations ......................................................................... 5.1

5.1.1 Conservation Effect of Consumer Information and Feedback Systems ................... 5.1

5.1.2 Deployment of Diagnostics in Residential and Small/Medium Commercial Buildings .................................................................................................... 5.1

5.1.3 Joint Marketing of Energy Efficiency and Demand Response Programs ................ 5.2

5.1.4 Measurement \& Verification for Energy Efficiency Programs ............................... 5.2

5.1.5 Shifting Load to More Efficient Generation .................................................... 5.2 
5.1.6 Support Additional Electric Vehicles and Plug-In Hybrid Electric Vehicles .......... 5.2

5.1.7 Conservation Voltage Reduction and Advanced Voltage Controls ........................ 5.3

5.1.8 Support Penetration of Solar Generation (Renewable Portfolio Standard > 20\%) ... 5.3

5.1.9 Support Penetration of Renewable Wind Generation (Renewable Portfolio

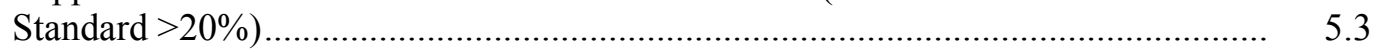

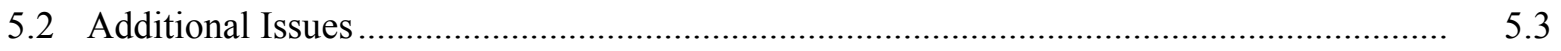

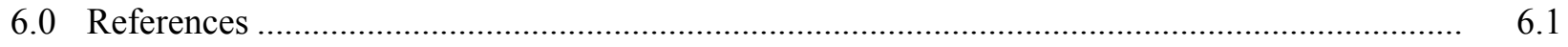

Attachment 1 Mechanism Review and Analysis .................................................................... A.1

Mechanism A: Conservation Effect of Consumer Information and Feedback Systems............. A.1

Mechanism B: Joint Marketing of Energy Efficiency and Demand Response Programs .......... B. B.1

Mechanism C: Deployment of Diagnostics in Residential and Small/Medium Commercial Buildings ............................................................................................ C. C.1

Mechanism D: Measurement \& Verification for Energy Efficiency Programs......................... D. 1

Mechanism E: Shifting Load to More Efficient Generation...................................................... E.1

Mechanism F: Support Additional Electric Vehicles and Plug-In Hybrid Electric Vehicles ..... F.1

Mechanism G: Conservation Voltage Reduction and Advanced Voltage Control.................... G.1

Mechanism H: Support Penetration of Solar Generation (RPS > 25\%) .................................. H.1

Mechanism I: Wind Energy Integration ....................................................................... I.1

Attachment 2 Electricity and $\mathrm{CO}_{2}$ Reduction Calculations …................................................. 2.1 


\section{Figures}

2.1 Today's Electricity Delivery System …......................................................................... 2.1

2.2 Defining the Smart Grid in Terms of Assets and Functions................................................... 2.3

2.3 The Business Case: Weighing the Capital Investments for Assets vs. the Value Streams from the Functions They Support........................................................................................ 2.8

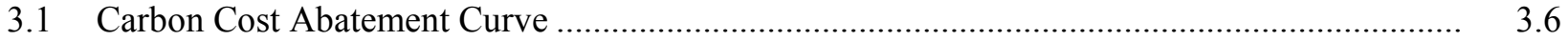

3.2 Monthly Energy Bills from Typical Home in a Hot Desert Climate ...................................... 3.12

3.3 Monthly Energy Bills from Typical Home vs. Monthly Average Temperature ....................... 3.13

3.4 Hourly Load Data vs. Temperature, One Plot for Each Hour ................................................. 3.14

3.5 Non-Linear Models of Three End-Use Load Subtotals for Hour 15 .......................................... 3.15

3.6 Load Duration Curve and Carbon Dispatch of a Typical Coal-Based Utility .......................... 3.23

3.7 Voltage Drop Along a Feeder at Peak and Minimum Loads ................................................. 3.27

3.8 Effect of Reactive Power Control on Voltage Drop................................................................ 3.28

3.9 Annual Fraction of Energy from Residential Solar PV at Which Reverse Power Flow

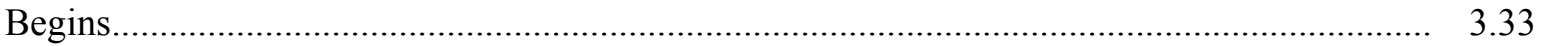

4.1 Climate Group Report: Policy Recommendations ...........................................................

4.2 How Green is the Smart Grid? Conservative and Expanded Scenarios ................................... 4.4 


\section{Tables}

3.1 Smart Grid Mechanisms and Impacts Analyzed ............................................................... 3.2

3.2 Potential Energy Consumption and Carbon Emissions Reductions from Smart Grid Deployment.

3.3 Estimated Direct Impacts of the Conservation Effect of Consumer Information and Feedback Systems.....

3.4 Estimated Indirect Impacts of Jointly Marketing Energy Efficiency and Demand Response Programs

3.5 Estimated Direct Impacts for Enabling Mass Deployment of Diagnostics in Residential and Small/Medium Commercial Buildings.

3.6 Estimated Indirect Impacts of Measurement \& Verification for Energy Efficiency Programs Leveraging a Smart Grid....

3.7 Estimated Direct Impacts of Measurement \& Verification for Energy Efficiency Programs Leveraging a Smart Grid.

3.8 Estimated Direct Utility Energy and Carbon Reductions for Shifting Load to More Efficient Generation.

3.9 Estimated Direct Utility Energy and Carbon Reductions from Supporting Additional Electric Vehicles and Plug-In Hybrid Electric Vehicles....

3.10 Estimated Direct Utility Energy and Carbon Reductions for Conservation Voltage Reduction and Advanced Voltage Control.

3.11 Estimated Direct Impacts of Reduced Energy Needed to Supply Regulation for Wind Energy Penetration at 25\% RPS.

3.12 Estimated Indirect Impacts of Reduced Needed Reserve Capacity for Wind Energy Penetration at $25 \%$ RPS

4.1 EPRI Report: Smart Grid Energy Savings and Avoided $\mathrm{CO}_{2}$ Emissions Summary.

4.2 Climate Group Report: Smart Grid Energy Savings and Avoided $\mathrm{CO}_{2}$ Emissions Summary ...

4.3 Smart Grid Energy Savings and Avoided $\mathrm{CO}_{2}$ Emissions Summary

4.4 Comparison of Estimated Reductions in Energy Consumption and $\mathrm{CO}_{2}$ Emissions 


\subsection{Introduction}

The U.S. Department of Energy (DOE), Office of Electricity Delivery and Energy Reliability's (OE's) mission is "...to lead national efforts to modernize the electric grid; enhance security and reliability of the energy infrastructure; and facilitate recovery from disruptions to energy supply." One key element of OE's strategy for modernizing the electric grid (http://www.oe.energy.gov/1165.htm) is to take advantage of the potential for information technology to change the operational and control strategies it uses to help keep electricity affordable by improving the cost-effectiveness of grid infrastructure investments and increasing the reliability of electricity supply and delivery to customers. OE has played a leading role in identifying this opportunity, which has come to be known generically as the "smart grid," by articulating its benefits to industry, policy makers, customers, and other stakeholders, by advancing key technologies and funding field demonstrations to prove its performance advantages (http://www.oe.energy.gov/smartgrid.htm).

As part of its efforts to quantify benefits from the smart grid, OE's Smart Grid Research and Development Program tasked Pacific Northwest National Laboratory (PNNL) to develop an estimate of the potential energy and carbon benefits that will result from deployment of the smart grid. The goals of this project are to:

- Define the mechanisms by which the smart grid can contribute to energy efficiency and the integration of renewable generation to provide carbon benefits to the United States.

- Quantify, to the extent possible, those benefits and contributions in terms of reductions in electricity consumption and $\mathrm{CO}_{2}$ emissions.

This report articulates nine mechanisms by which the smart grid can reduce energy use and carbon impacts associated with generating and delivering electricity. To the extent possible, it presents quantitative estimates of potential impacts for each of the mechanisms through a detailed search of published results and by conducting simple analyses of the potential effects. This report does not attempt to justify the cost effectiveness of the smart grid, which to date has been based primarily upon the twin pillars of cost-effective operation and improved reliability. Rather, it attempts to quantify the additional benefits inherent in the smart grid's potential contribution to the nation's goal of mitigating climate change by reducing the carbon footprint of the electric power system.

OE's smart grid effort, formally established by the Energy Independence and Security Act (EISA) of 2007, is characterized by the 10 points shown in the text box (EISA 2007). The electricity and $\mathrm{CO}_{2}$ reductions that may be obtained by implementing smart grid technologies estimated in this report will help identify the benefits associated with goals $3,4,7$, and 8 . In addition, the assessment provides a number of recommendations and issues to consider in the formulation and conduct of OE's research program that addresses: 1) technology development to modernize the delivery of electricity, 2) policy coordination and implementation to facilitate electricity system modernization, and 3) the ability for stakeholders to prepare for and respond to electricity supply disruptions. 


\section{Energy Independence and Security Act of 2007 Sec. 1301. Policy on Modernization of Electricity Grid}

... support the modernization of the Nation's electricity transmission and distribution system to maintain a reliable and secure electricity infrastructure that can meet future demand growth and to achieve each of the following, which together characterize a smart grid:

(1) Increased use of digital information and controls technology to improve reliability, security, and efficiency of the electric grid.

(2) Dynamic optimization of grid operations and resources, with full cyber-security.

(3) Deployment and integration of distributed resources and generation, including renewable resources.

(4) Development and incorporation of demand response, demand-side resources, and energy-efficiency resources.

(5) Deployment of "smart" technologies (real-time, automated, interactive technologies that optimize the physical operation of appliances and consumer devices) for metering, communications concerning grid operations and status, and distribution automation.

(6) Integration of "smart" appliances and consumer devices.

(7) Deployment and integration of advanced electricity storage and peak-shaving technologies, including plug-in electric and hybrid electric vehicles, and thermal-storage air conditioning.

(8) Provision to consumers of timely information and control options.

(9) Development of standards for communication and interoperability of appliances and equipment connected to the electric grid, including the infrastructure serving the grid.

(10) Identification and lowering of unreasonable or unnecessary barriers to adoption of smart grid technologies, practices, and services.
Related assessments by the Electric Power Research Institute (EPRI) and The Climate Group, and an article in The Electricity Journal, also examined the electricity and $\mathrm{CO}_{2}$ benefits that may result from implementation of the smart grid (EPRI 2008; GeSI 2008; Hledik 2009). These assessments also provide first-order estimates of the energy and carbon benefits for the emerging smart grid area and provide useful comparative benchmarks for this effort.

The report is organized into five sections. Section 2.0 provides an overview of the current electrical grid and a definition of the smart grid with its costs and benefits. Section 3.0 presents the assessment methodology, summarizes each mechanism and the results of its assessment, and Section 4.0 compares the results from EPRI and The Climate Group studies, and The Electricity Journal article. Further details of the assessments for the mechanism are provided in Attachments 1 and 2. The last section (Section 1.0) provides recommendations on mechanisms and benefits that deserve further exploration. 


\subsection{Smart Grid - What it Is, What it Does, and Who it Benefits}

A basic perspective of this analysis is that, over the next 20 years, smart grid technology will become pervasive in the United States because of the cost efficiencies it provides for the electric power system, and that it could be leveraged to provided additional benefits of reduced energy consumption and carbon emissions. Therefore, it is important to understand the kinds of assets involved in a smart grid and how they are functionally engaged to provide cost efficiencies. This sets the context for why a smart grid is likely to be deployed and what assets it is likely to contain that can be leveraged for these additional environmental benefits. The discussion in this section attempts to outline this perspective.

Electricity has historically been generated at central station power plants and distributed to customers, as shown in Figure 2.1. In 2007, an estimated $995 \mathrm{GW}$ of generating capacity delivered 4.2 GWh to 142 million customers (DOE/EIA 2009) over approximately 158,000 miles of transmission line $>230 \mathrm{kV}$ (DOE 2002). Estimates of distribution lines are in the range of 1 million miles. The voltage is steppedup from large central generating stations for transmission through 10,287 transmission stations, steppeddown for utility distribution in 2,178 distribution substations (DOE/OE 2006), may be further steppeddown at points along the utility distribution lines (feeders), and again at pad- and pole-mounted transformers to provide low-voltage service to one or a several customers.

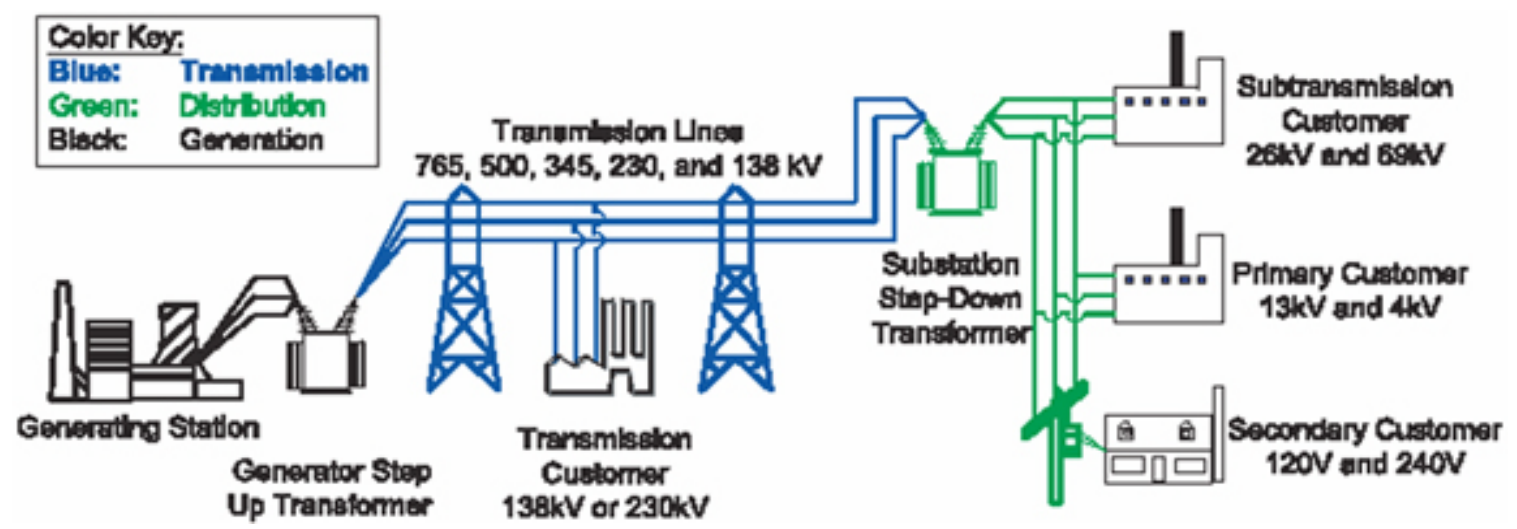

Figure 2.1. Today's Electricity Delivery System (Source: DOE/FEMP [2009], Electricity 101 at http://www.oe.energy.gov/information_center/electricity101.htm)

The delivery of electricity typically utilizes a supervisory control and data acquisition system (SCADA) that provides monitoring and control from generation through the step-down substation to detect the need for an increase/reduction in generating resources, and to respond to system instabilities. Key limitations of SCADA systems are the following:

- limited bandwidths and relatively slow data transmission rates that often require several seconds or more to respond to an alarm or system change

- limited or no visibility in the distribution network below the substation.

The coming evolution in the delivery of electricity is the smart grid, which is the application of information technology that enables more visibility and control of both the existing grid infrastructure and 
new grid assets, such as customer demand response and distributed energy resources consisting of small generators and electricity storage devices. The smart grid's much higher fidelity control is provided through high-speed, two-way communication, sensing, and real-time coordination of all assets down to the customer meter and the end-use devices. Thus the smart grid is not characterized by a single technology or a device, but instead is a vision for a distributed, internet-like system that will:

- provide better control of existing grid infrastructure assets

- provide additional functionality and benefits from existing assets

- integrate new (often small, widely distributed) assets into the existing operational paradigm

- engage these new assets to provide entirely new benefits to the grid.

The next immediate developments in SCADA technology for utilities are to increase bandwidth and begin to measure and control assets below the substation level, at which time the system will begin to become part of a distributed control system (Boyer 2007) — and a key part of the smart grid.

This vision is perhaps best described by a set of essential characteristics, or outcomes (see box).

"The smart grid isn't a thing but rather a vision... It must be more reliable...more secure...more economic...more efficient...more environmentally friendly...(and) It must be safer. A "smart grid" can be (characterized as) a "transactive" agent...(that) will:

- Enable active participation by consumers...

- Accommodate all generation and storage options...

- Enable new products, services, and markets...

- $\quad$ Provide power quality for the digital economy...

- Optimize asset utilization and operate efficiently...

- Anticipate and respond to system disturbances (selfheal).

- Operate resiliently against attack and natural disaster.

Achieving the vision is dependent upon participant circumstances and involves:

- Empowering consumers by giving them the information and education they need to effectively utilize the new options provided by the smart grid...

- Improved reliability and "self-healing" of the distribution system...

- Integration of the transmission and distribution systems to enable improved overall grid operations and reduced transmission congestion...

- Integration of the grid intelligence acquired to achieving with new and existing asset management applications...

Source: Smart Grid News, April 22, 2009. What is the smart grid?
Beyond describing the smart grid as a vision, it is helpful to describe what the smart grid consists of in terms of

- the assets that would be purchased

- the functions for which they would be used, and from which benefits are derived.

This is illustrated in the matrix in Figure 2.2, with a number of key assets on the horizontal axis and broadly defined categories of major functions on the vertical axis. This illustration of the current and emerging vision for the smart grid is not intended to be definitive or comprehensive, but rather will evolve over time.

Assets are divided into primary and enabling assets. Primary assets are the smart grid's “prime movers," i.e., non-traditional assets that are actively controlled to effect change in the grid's operating conditions. 


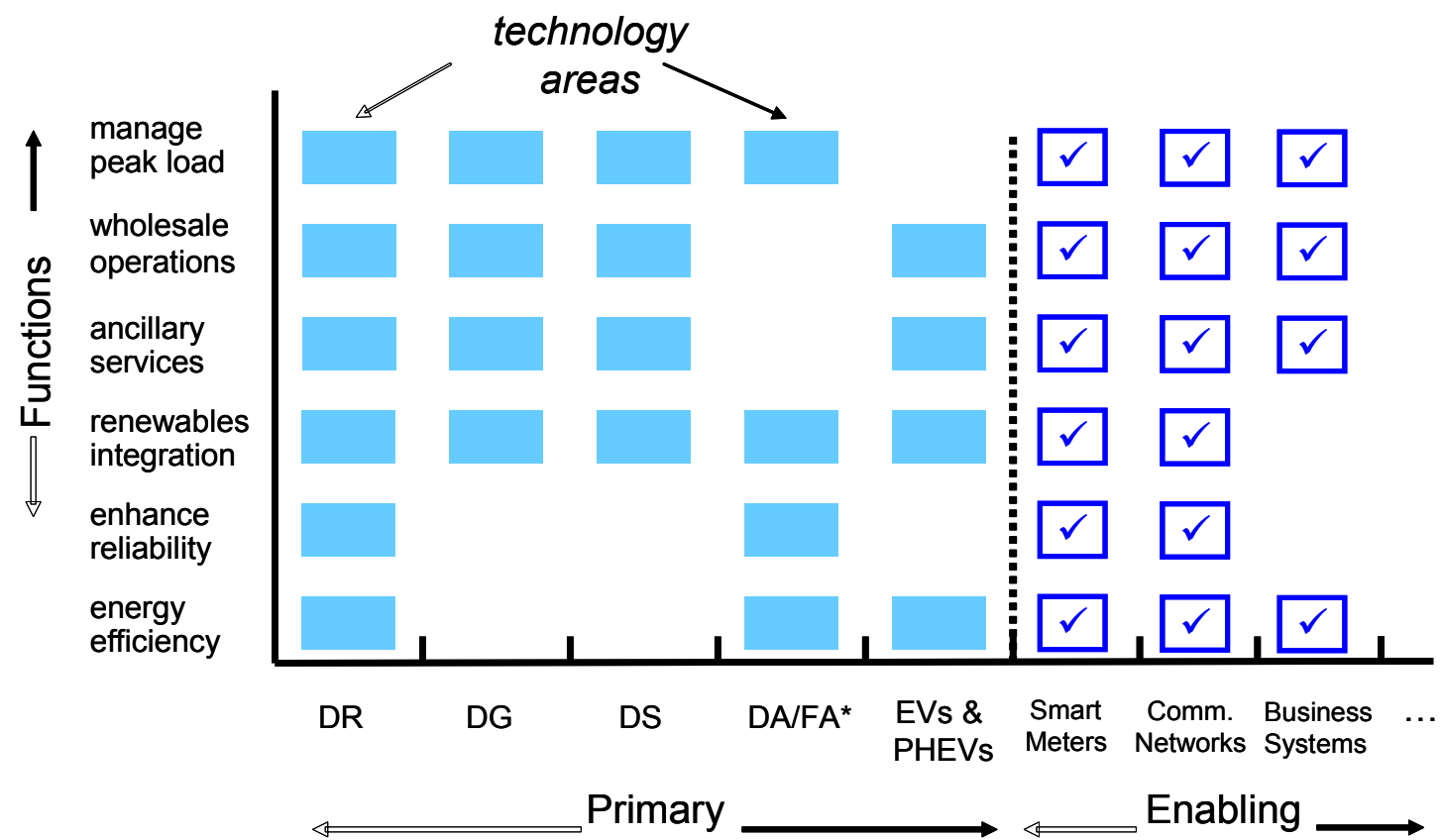

$\mathrm{DR}=$ demand response, $\mathrm{DG}=$ distributed generation, $\mathrm{DS}=$ distributed storage, $\mathrm{DA} / \mathrm{FA}=$ distribution automation/feeder automation, EVs \& PHEVs = electric vehicles/plug-in hybrid electric vehicles

Figure 2.2. Defining the Smart Grid in Terms of Assets and Functions

Enabling assets are the sensing, software, and information infrastructure required to coordinate the operation of the primary assets to respond to grid conditions. Although more accurately portrayed as a separate third dimension, enabling assets are shown here on the same axis for clarity.

Functions are grid operational strategies that use smart grid assets to derive cost, reliability, and efficiency or renewable energy benefits. The intersection of an asset and a function, denoted as a technology area, is the set of policies, engagement strategies, incentive mechanisms, control strategies, software applications, and capabilities of the primary and enabling assets required to accomplish a given function. The specific technology areas in Figure 2.2 illustrate the asset-function intersections and do not attempt to be definitive.

\subsection{Primary Assets}

The primary assets in Figure 2.2, broadly considered key to the smart grid, are:

- Demand response (DR) - communications and controls for end-use devices and systems to reduce (or, in special cases, increase) their demand for electricity at certain times.

- Distributed generation (DG) - small engine or turbine generator sets, wind turbines, and solar electric systems connected at the distribution level.

- Distributed storage (DS) - batteries, flywheels, super-conducting magnetic storage, and other electric and thermal storage technologies connected at the distribution level.

- Distribution/feeder automation (DA/FA) - distribution and feeder automation expand SCADA communications in substations and into the feeders with remotely actuated switches for reconfiguring 
the network, advanced protective relays with dynamic and zonal control capabilities, dynamic capacitor bank controllers, and condition-based transformer-management systems (to name a few).

- Transmission wide-area visualization and control - transmission control systems that rapidly sense and respond to disturbances.

- Electric and plug-in electric hybrid vehicles (EVs/PHEVs) - the batteries in EVs represent both a new type of load that must be managed and an opportunity for them to discharge as energy storage resources to support the grid.

Demand response is intentionally defined as an asset, to differentiate the investment required for installing its control and communications capabilities from its $\boldsymbol{u} \boldsymbol{e} \boldsymbol{e}$ to achieve one or more functions. Although we recognize that the term demand response is often used to represent both the asset and its use for the peak load management function, this is more precisely the technology area represented by the intersection of the demand response asset and the peak load-management function. This distinction between demand response as an asset and the functions it can provide is helpful because demand response, like many other smart grid assets, can provide a number of other functional benefits ranging from ancillary services to reliability. Along with distributed generation and storage, demand response can play a key role in providing the additional ancillary services and reliability required for effectively integrating renewables. Additionally, as discussed in Section 3.0, there is potential for the control signals that support demand response to be used for conducting end-use system diagnostics and improving feedback to consumers to obtain energy efficiency.

The notion of active control in response to grid conditions is foundational to the notion of a smart grid. Most energy efficiency investments are passive in that they require no control at all (better insulation or air conditioner efficiency, for example). Some forms of active energy efficiency are controls-based (e.g., thermostat setbacks, clothes dryer humidity controls) but are not designed to be responsive to grid conditions. Hence, energy efficiency investments, while critical to obtaining efficiency and carbon savings, are not smart grid assets in this framework. However, this report does consider obtaining efficiency benefits as a functional objective for the use of smart grid assets.

Similarly, renewables themselves are not generally envisioned as a controllable smart grid asset. ${ }^{1}$ The carbon-free energy they supply is critical to achieving the nation's carbon-management goals, however. One of the functions of a smart grid is the ability to manage the assets under its control to help integrate renewables, such as mitigating the need for additional costly ancillary services to manage their intermittency, and reducing costs for improved voltage control schemes and short-circuit protection.

\footnotetext{
${ }^{1}$ However, the power factor of the output from the inverters for renewable generators could be managed to meet the reactive power needs of the grid.
} 


\subsection{Enabling Assets}

Investments in a number of enabling assets are also necessary to support the use of the primary assets for smart grid applications, hence the function of a smart grid. Among these cross-cutting technologies are:

- wide-area communications networks, servers, gateways, etc.

- smart meters-beyond what many consider as basic advanced metering infrastructure (AMI) technology, a more fully smart meter could also

- support shorter metering intervals approaching 5 minutes or less to support provision of ancillary services and distribution capacity management (rather than the hourly interval generally considered adequate for peak load management at the bulk power systems level)

- full two-way communications including to a home-area network to communicate to smart thermostats and appliances

- instantaneously read voltage, current, and power factor to support distribution state estimation and optimized system volt-VAR control

- offer remote connect/disconnect functionality for reliability and customer service applications

- local-area home, commercial building, and industrial energy management and control systems (EMCS) and networks

- consumer information interfaces and decision support tools

- utility back-office systems, including billing systems.

Other key technical ingredients of the smart grid that are similarly cross-cutting, but are typically embedded in assets are:

- cyber-security technologies for secure communications for all levels of operation

- an interoperability framework, and associated standards and protocols that focus on communications between the various SCADA control domains inherent in the smart grid: including the Independent System Operator/Regional Transmission Organization utility, customer, and aggregator.

\subsection{Functions: Operational Objectives}

Functions are the benefits or applications to which smart grid assets are engaged to improve cost effectiveness, reliability, and energy efficiency of the power system. These can be summarized in broad categories corresponding to the benefits derived:

- managing peak load capacity for generation, transmission, and distribution

- reducing costs for wholesale operations

- providing enhanced reliability/adequate reliability at less cost

- providing ancillary services 
- reducing the operational costs of integrating renewables

- leveraging the network for energy efficiency and carbon savings.

The first four function categories have long been considered central elements of the smart grid and are briefly described here. The last two are the fundamental subjects of this report and are discussed in Section 3.0.

Managing peak load capacity includes displacing the need for new generation, localizing this function to displace the need for new transmission, further localizing it to manage capacity to offset the need for new and upgraded distribution substations and feeders, and managing transformer loading to extend their lifetimes. About $40 \%$ of grid infrastructure costs are for generation capacity, which must be adequate to serve peak load demand while maintaining adequate reserves for forced outages and contingencies. In light of growing demand for generation worldwide, environmental constraints on new coal generation, the imposition of renewable generation portfolios by states, and rising costs for steel, concrete, and other materials, and costs for new generation capacity to meet load growth are expected to grow substantially. Another $40 \%$ of infrastructure costs are for distribution systems, so the opportunity to manage peak load demand at the substation level is an important opportunity. Peak load management from demand response, distributed storage, and optimization of distribution delivery voltages and power factors can all serve to defer investment in generation, transmission, and distribution systems. The value stream from this is derived in terms of the avoided carrying costs for investment in new capacity.

Reducing costs for wholesale operations involves lowering the demand for generation when marginal production costs are greater than revenues from retail sales, similarly minimizing purchases or maximize production when wholesale prices are high, and reducing transmission loads when and where congestion costs are high. This can be accomplished by utilizing demand response, distributed storage, and distribution voltage controls to reduce net demand.

"Through proactive grid management and automated response, the frequency and duration of power outages can be reduced, which will result in fewer anxious calls to utility call centers and improved consumer satisfaction. Remote monitoring and control devices throughout the system can create a "self-healing" grid, which can restore and prevent outages and extend the life of substation equipment and distribution assets. Through such automation, rising consumer expectations for power quality and reliability can be met in the face of growing electricity demand and an aging infrastructure and workforce."

Source: EAC (2008).
Enhanced reliability. A smart grid can enhance reliability in two fundamental ways. It can prevent and limit blackouts with transmission wide-area control and visualization tools that enhance situational awareness and rapidly reconfigure the transmission grid to prevent or limit a blackout. At the distribution level, where the vast bulk of outages occur in terms of aggregate customerminutes without power, outages are typically caused by events such as vehicle accidents, wind and ice storms, and animals shorting out transformers, rather than systemic failures. To remedy these outages, distribution and feeder automation assets can be used to rapidly isolate faults and then reconfigure distribution feeders through remotely actuated switches. This shortens the recovery time for nearly all customers from an hour or more to a matter of seconds. In its ultimate form, this is a stand-alone microgrid fully capable of supply its own power and managing its local distribution. 
Ancillary services. Beyond power production, many services are provided by power plants to keep the grid in a stable and reliable condition. These include the following:

- Regulation is supplied on a minute-by-minute basis to control the supply/demand balance by continually throttling variable-output power plants.

- Ramping and load following are similarly required to manage the grid when the rate of load change is high, such as the morning and late evening.

- Spinning and non-spinning reserve capacity is required to manage the sudden, forced-outage loss of power plants scheduled to generate electricity on a given day.

- Reactive power needs to be supplied by power plants to correct phase shifts between current and voltage due to system load variance.

The highest cost resources in power markets that quantify such services are those for short-term regulation. Today, we turn power plants up and down continually to provide regulation, which wastes fuel and increases wear and tear on the plants. Ancillary services could be supplied by dispatching the smart grid's demand response, distributed generation, and storage assets to provide regulation and load following services, and using them in standby mode (when not otherwise engaged) to provide spinning reserves. While valuable in today's grid operations, the need for ancillary services is projected to increase as large amounts of renewable generation penetrates the grid, due to the intermittency of output from wind and solar generators.

\subsection{The Business Case for a Smart Grid}

The matrix of assets and functions forms a useful basis for describing the business case for the smart grid. In essence, the business case for a smart grid weighs the capital investments in an asset or set of assets against the multiple value streams that can be derived from the applications they support (Figure 2.3). The business case is successful when the sum of the value streams derived are greater than the capital investments required, less an incentive offered to engage customer or third-party assets.

It is important to note that any given asset can support a number of functions (as illustrated with demand response), and that any given function (such as managing peak load) can be supported by a variety of assets. Therefore, smart grid assets can literally work together or compete with each other (and traditional infrastructure) to provide the necessary functions. This suggests that not only must a primary asset and its enabling assets be cost effective, it must also be more cost effective that its competitors. It also suggests, as has been pointed out by many observers, that the smart grid's ultimate configuration is necessarily path-dependent, at least to a degree, with respect to the order in which assets are deployed.

The economics of the smart grid are difficult to analyze, but the business case is gradually becoming clearer and the smart grid vision is becoming a reality. Early evidence is the passage of EISA in 2007, demonstrations that showed the viability of the smart grid concept, and Xcel Energy's initiation of Boulder, Colorado's SmartGridCity (http://smartgridcity.xcelenergy.com) project in 2007. In some utilities and states, investments in AMI and demand response assets were justified to regulators and underway before the DOE infrastructure and demonstration grant programs were announced. 


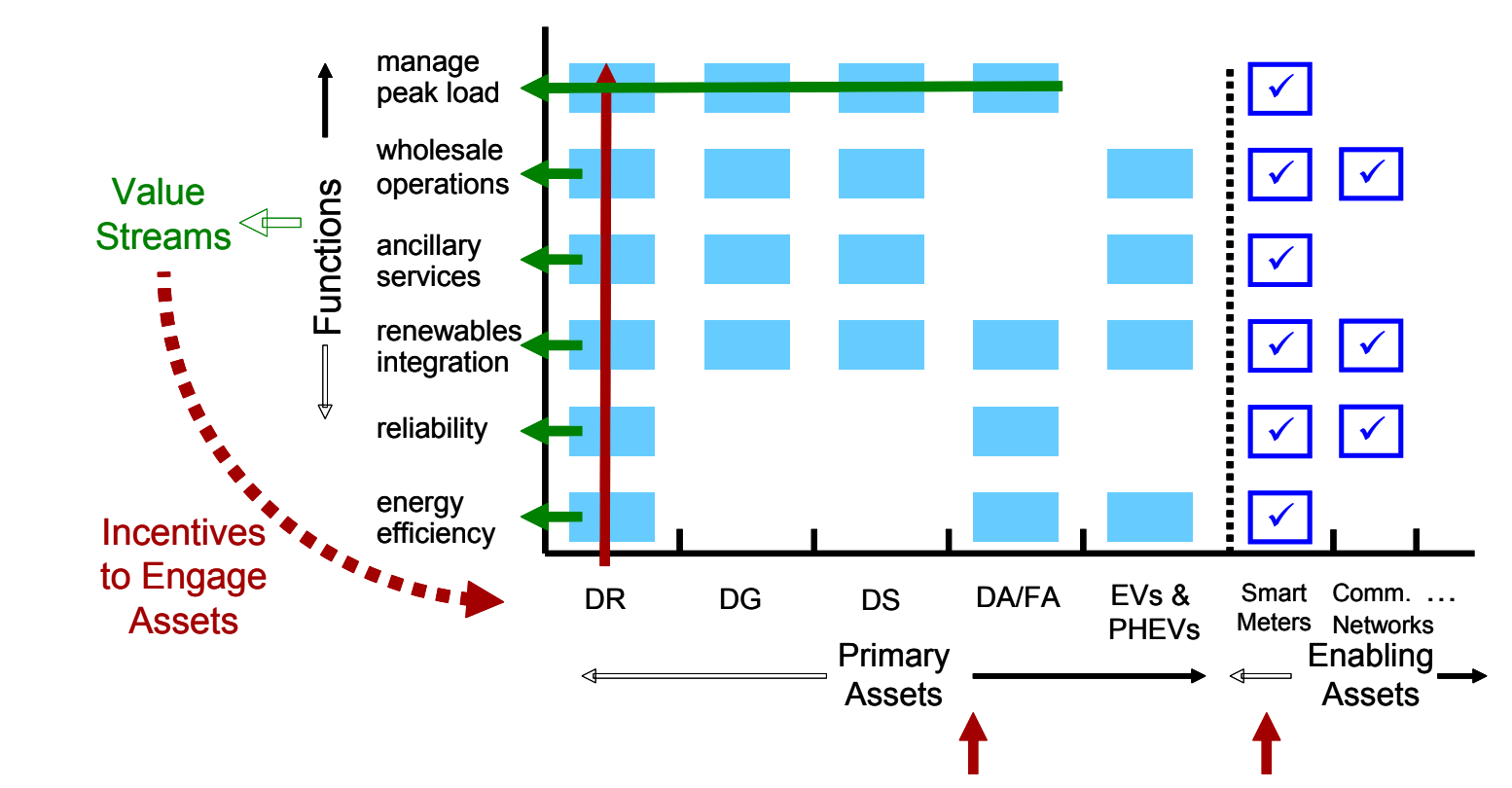

$\mathrm{DR}=$ demand response, $\mathrm{DG}=$ distributed generation, $\mathrm{DS}=$ distributed storage, $\mathrm{DA} / \mathrm{FA} \ddot{=}$ distribution automation/feeder automation, EVs \& PHEVs = electric vehicles/plug-in hybrid electric vehicles

Figure 2.3. The Business Case: Weighing the Capital Investments for Assets vs. the Value Streams from the Functions They Support

More recently, DOE released announcements to fund modernization of grid infrastructure and conduct demonstrations as part of EISA. One of the goals of the infrastructure grants is to spur mass production and deployment so that costs at scale can be determined. The goal of the demonstration grants is to build the business case by expanding the scope of smart grid functions into unproven areas, and quantifying their benefits. Commensurate with identification and quantification of the energy and environmental benefits, efforts are also underway to improve the monetization and allocation of the economic benefits to stakeholders.

The literature describing the smart grid concept, operation, and benefits is growing. A number of documents that provide the reader a more detailed discussion of the concept and the multi-faceted benefits are:

- DOE. 2008. The Smart Grid: An Introduction

- Electric Advisory Committee. 2008. Smart Grid: Enabler of the New Energy Economy

- Pacific Northwest National Laboratory. 2007. Pacific Northwest GridWise ${ }^{\mathrm{TM}}$ Testbed Demonstration Projects, Part I. Olympic Peninsula Project

- EPRI. 2008. The Green Grid: Energy Savings and Carbon Emissions Reductions Enabled by a Smart Grid. Electric Power Research Institute, Palo Alto, California: 2008. 1016905.

This report does not attempt to monetize and include the energy and carbon-management benefits into the business case to help justify a smart grid. Rather, smart grid deployment will be justified on operational merits in its early stages, and the additional benefits treated here provide an enhancement to this value at little or no additional cost. The associated marginal costs are expected to be low, because 
these enhancements are primarily in the form of software applications or control algorithms, while the primary costs of smart grid are for the purchase and deployment of the assets involved. If the business case for a smart grid is not made without including the additional energy and carbon benefits, then the additional value provided by the carbon benefits can be included as the uncertainties of these benefits becomes better understood.

The goal of this report is to translate these additional benefits into reductions in energy consumption and $\mathrm{CO}_{2}$ emissions that will accrue to customers, utilities, and society. This report and the three others reviewed provide a first-order assessment of these potential benefits from deployment of smart grid technologies. Improved understanding of these benefits will require a more significant effort to account for the displacement of generating resources by renewables, among other issues. 



\subsection{Mechanism Methodology and Summaries}

Nine mechanisms, as shown in Table 3.1, by which a smart grid can help reduce energy consumption and carbon emissions are described in this report. Two types of impacts are analyzed: 1) direct reductions, in which smart grid functions produce savings in energy and/or emissions consumed at the end-use or by reducing generation requirements, and 2) indirect reductions in which smart grid functions produce cost savings, which are subsequently reinvested in energy efficiency and/or renewable resources. As discussed earlier, no attempt has been made to quantify impacts on consumer electric bills, utility revenue requirements, or other economic considerations that are considered the fundamental benefits of a smart grid.

Indirect mechanisms do not result in energy and emission savings in and of themselves. Rather, they reduce capital and/or operational costs that can then be reinvested in the deployment of energy efficiency programs or of renewables to provide reductions. To estimate the potential value of indirect reductions and place them in context with the direct reduction estimates, we estimate the savings that would ensue from reinvesting the cost savings in the purchase of additional cost-effective energy efficiency at an average electricity cost of $8.8 \notin / \mathrm{kWh}$. In effect, this represents a policy decision to reinvest the savings in the purchase of additional efficiency and renewable resources.

An alternative policy decision is to "pocket" these capital and operational cost savings, in effect using them to reduce the societal cost of obtaining reductions from energy efficiency and renewables that would have been purchased anyway. One view of the consequences of such policy is that no indirect benefits would be realized. Another view is that additional deployment of such resources would naturally occur because they are effectively cheaper, and the potential indirect reductions are a way to estimate this effect. Hence, the potential value of indirect reductions is subjective and left to the reader.

Table 3.2 provides an overview of the estimated potential of a smart grid to reduce energy consumption and $\mathrm{CO}_{2}$ emissions from the nine mechanisms examined. Each mechanism was assigned to a subject matter expert, who conducted a review of the applicable literature. The potential impacts are primarily based on the results found in the literature and the judgment of the authors regarding key assumptions, as documented in Attachments 1 and 2. Table 3.2 first lists the potential direct reductions, and then the potential indirect reductions, for each mechanism. The second column is the estimated potential to reduce the annual electricity supply in 2030 for a specific subsector of the United States (columns five and six). The third and fourth columns provide low and high ranges for the estimate. The fuel consumption of light-duty vehicles (LDVs) (for Mechanism F) has been converted to its electricity equivalent so it can be viewed on an equivalent basis with the other mechanisms. No rigorous attempt has been made to analyze the uncertainty associated with each mechanism, because the methodology used is not tailored to provide such specific estimates. Instead, likely ranges of uncertainty are provided based on the judgment of the authors, in light of the range of results found in the literature and uncertainties in key assumptions. 
Table 3.1. Smart Grid Mechanisms and Impacts Analyzed

\begin{tabular}{|c|c|c|c|}
\hline \multirow{2}{*}{$\begin{array}{l}\text { Category of } \\
\text { Smart Grid } \\
\text { Function }\end{array}$} & \multirow[b]{2}{*}{ Mechanism } & \multicolumn{2}{|c|}{ Type of Impact Analyzed } \\
\hline & & $\begin{array}{c}\text { Direct Reduction of } \\
\text { Energy and } \mathrm{CO}_{2} \text { Emissions }\end{array}$ & $\begin{array}{c}\text { Indirect Reduction of } \\
\text { Energy and } \mathrm{CO}_{2} \text { Emissions }\end{array}$ \\
\hline $\begin{array}{l}\text { Energy } \\
\text { Efficiency }\end{array}$ & $\begin{array}{l}\text { A. Conservation Effect of } \\
\text { Consumer Information } \\
\text { and Feedback Systems }\end{array}$ & $\begin{array}{l}\text { Conservation Effect of Consumer } \\
\text { Feedback Based on AMI and } \\
\text { Demand Response Controls }\end{array}$ & -- \\
\hline $\begin{array}{l}\text { Energy } \\
\text { Efficiency }\end{array}$ & $\begin{array}{l}\text { B. Joint Marketing of } \\
\text { Energy Efficiency and } \\
\text { Demand Response } \\
\text { Programs }\end{array}$ & -- & $\begin{array}{l}\text { Energy efficiency program cost } \\
\text { savings from shared marketing } \\
\text { and outreach expenses }\end{array}$ \\
\hline $\begin{array}{l}\text { Energy } \\
\text { Efficiency }\end{array}$ & $\begin{array}{l}\text { C. Deployment of } \\
\text { Diagnostics in } \\
\text { Residential and Small/ } \\
\text { Medium Commercial } \\
\text { Buildings }\end{array}$ & $\begin{array}{l}\text { Efficiency savings from } \\
\text { equipment performance } \\
\text { diagnostics for heating, } \\
\text { ventilating, and air conditioning } \\
\text { (HVAC), and lighting }\end{array}$ & -- \\
\hline $\begin{array}{l}\text { Energy } \\
\text { Efficiency }\end{array}$ & $\begin{array}{l}\text { D. Measurement \& } \\
\text { verification }(\mathrm{M} \& \mathrm{~V}) \text { for } \\
\text { Energy Efficiency } \\
\text { Programs }\end{array}$ & $\begin{array}{l}\text { Efficiency from marginal energy } \\
\text { efficiency measures that are cost } \\
\text { effective based on more accurate } \\
\mathrm{M} \& \mathrm{~V}\end{array}$ & $\begin{array}{l}\text { Reduced costs for } \mathrm{M} \& \mathrm{~V} \text { of } \\
\text { savings from energy efficiency } \\
\text { programs }\end{array}$ \\
\hline $\begin{array}{l}\text { Energy } \\
\text { Efficiency }\end{array}$ & $\begin{array}{l}\text { E. Shifting Load to More } \\
\text { Efficient Generation }\end{array}$ & $\begin{array}{l}\text { Reduced fuel and emissions } \\
\text { resulting from load shifting to } \\
\text { more energy-efficient power } \\
\text { plants using demand response and } \\
\text { distributed storage }\end{array}$ & -- \\
\hline $\begin{array}{l}\text { Energy } \\
\text { Efficiency }\end{array}$ & $\begin{array}{l}\text { F. Support Additional EVs } \\
\text { and PHEVs }\end{array}$ & $\begin{array}{l}\text { Reduced fuel and emissions from } \\
\text { the additional electric-powered } \\
\text { LDVs enabled by smart charging }\end{array}$ & -- \\
\hline $\begin{array}{l}\text { Energy } \\
\text { Efficiency }\end{array}$ & $\begin{array}{l}\text { G. Conservation Voltage } \\
\text { Reduction and Advanced } \\
\text { Voltage Control }\end{array}$ & $\begin{array}{l}\text { Reduced distribution losses and } \\
\text { end-use energy consumption from } \\
\text { optimizing distribution voltage }\end{array}$ & -- \\
\hline $\begin{array}{l}\text { Renewables } \\
\text { Integration }\end{array}$ & $\begin{array}{l}\text { H. Support Penetration of } \\
\text { Solar Generation } \\
\text { (renewable portfolio } \\
\text { standard [RPS] }>20 \% \text { ) }\end{array}$ & $\begin{array}{l}\text { Distribution-level solar generation } \\
\text { enabled by using advanced voltage } \\
\text { controls and feeder automation to } \\
\text { manage reverse power flow }\end{array}$ & -- \\
\hline $\begin{array}{l}\text { Renewables } \\
\text { Integration }\end{array}$ & $\begin{array}{l}\text { I. Support Penetration of } \\
\text { Renewable Wind } \\
\text { Generation }(20 \% \text { RPS })\end{array}$ & $\begin{array}{l}\text { Reduced energy consumption } \\
\text { by using demand response and } \\
\text { distributed storage instead of } \\
\text { power plants to supply regulation } \\
\text { services }\end{array}$ & $\begin{array}{l}\text { Reduced costs for additional } \\
\text { generation capacity by using } \\
\text { demand response and distributed } \\
\text { storage instead of power plants } \\
\text { to meet reserve requirements }\end{array}$ \\
\hline
\end{tabular}


Table 3.2. Potential Energy Consumption and Carbon Emissions Reductions from Smart Grid Deployment

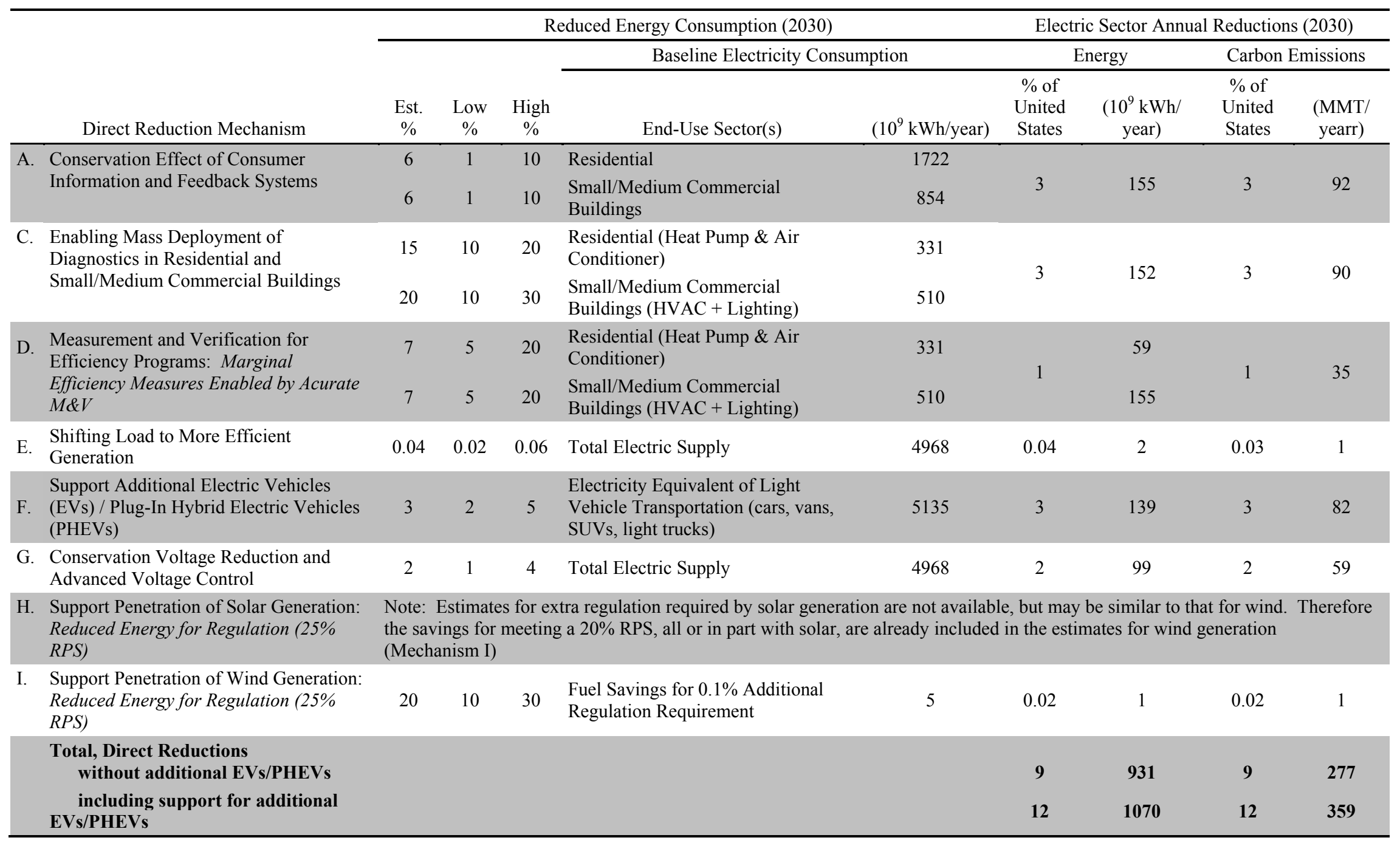


Table 3.2. (contd)

\begin{tabular}{|c|c|c|c|c|c|c|c|c|c|c|c|}
\hline & \multirow[b]{3}{*}{$\begin{array}{c}\text { Indirect Redcution Mechanism } \\
\text { (Reinvestment of Capital Savings in } \\
\text { Efficiency/Renewables) }\end{array}$} & \multicolumn{6}{|c|}{ Avoided Expenditure Reinvested to Save Carbon (2030) } & \multicolumn{4}{|c|}{ Electric Sector Annual Reductions (2030) } \\
\hline & & \multirow[b]{2}{*}{$\begin{array}{c}\text { Est. } \\
\%\end{array}$} & \multirow[b]{2}{*}{$\begin{array}{l}\text { Low } \\
\%\end{array}$} & \multirow[b]{2}{*}{$\begin{array}{c}\text { High } \\
\%\end{array}$} & \multicolumn{2}{|l|}{ Baseline Captial Expenditure } & \multirow{2}{*}{$\begin{array}{l}\text { Savings } \\
\left(10^{9} \$\right)\end{array}$} & \multicolumn{2}{|c|}{ Energy } & \multicolumn{2}{|c|}{ Carbon Emissions } \\
\hline & & & & & Investment & $\left(10^{9} \$\right)$ & & $\begin{array}{l}\% \text { of } \\
\text { United } \\
\text { States }\end{array}$ & $\begin{array}{l}\left(10^{9} \mathrm{kWh} /\right. \\
\text { year })\end{array}$ & $\begin{array}{l}\text { \% of } \\
\text { United } \\
\text { States }\end{array}$ & $\begin{array}{c}(\mathrm{MMT} / \\
\text { year) }\end{array}$ \\
\hline \multirow[t]{2}{*}{ B. } & $\begin{array}{l}\text { Joint Marketing of Efficiency and Demand } \\
\text { Response Programs }\end{array}$ & 0 & 0 & 1 & $\begin{array}{l}10 \% \text { Demand Response, Residential } \\
\text { @ } \$ 400 / \mathrm{kW} \& 8.8 \phi / \mathrm{kWh}\end{array}$ & 15 & 0.0 & & & & \\
\hline & & 0 & 0 & 1 & $\begin{array}{l}10 \% \text { Demand Response, } \\
\text { Small/Medium Commercial } \\
\text { Buildings @ } \$ 300 / \mathrm{kW} \& 8.8 \phi / \mathrm{kWh}\end{array}$ & 6 & 0.0 & 0 & 0 & 0 & 0 \\
\hline \multirow[t]{2}{*}{ D. } & \multirow{2}{*}{$\begin{array}{l}\text { Measurement and Verification for } \\
\text { Efficiency Programs: M\&V Cost Savings } \\
\text { for Energy Efficiency Programs }\end{array}$} & 1 & 0 & 2 & $\begin{array}{l}\text { 10\% Energy Efficiency, Residential } \\
\text { @ } 8.8 \notin / \mathrm{kWh}, 10 \text {-Year Life }\end{array}$ & 152 & 1.5 & \multirow[b]{2}{*}{0.5} & \multirow[b]{2}{*}{26} & \multirow[b]{2}{*}{0.5} & \multirow[b]{2}{*}{15} \\
\hline & & 1 & 0 & 2 & $\begin{array}{l}\text { 10\% Energy Efficiency, } \\
\text { Small/Medium Commercial } \\
\text { Buildings @ } 8.8 \notin / \mathrm{kWh}, 10 \text {-Year Life }\end{array}$ & 75 & 0.8 & & & & \\
\hline & $\begin{array}{l}\text { Support Penetration of Solar Generation: } \\
\text { Distribution Voltage Controls for RPS }> \\
\sim 20 \%\end{array}$ & \multicolumn{10}{|c|}{$\begin{array}{l}\text { Note: Voltage control for distribution systems with solar generation above } \sim 20 \% \text { RPS becomes problematic due to reverse flow } \\
\text { of power toward the substation. Comparative costs for voltage management alternatives are not available. }\end{array}$} \\
\hline \multirow[t]{4}{*}{ I. } & $\begin{array}{l}\text { Support Penetration of Wind Generation: } \\
\text { Reduced Reserve Capacity ( } 25 \% \text { RPS) }\end{array}$ & 2 & 1 & 3 & $\begin{array}{l}1111 \text { GW Total Generation Capacity } \\
\text { @ } \$ 1000 / \mathrm{kW}\end{array}$ & 1111 & 22 & 5 & 253 & 5 & 150 \\
\hline & Total, Indirect Reductions & & & & & & & 6 & 253 & 6 & 150 \\
\hline & $\begin{array}{l}\text { Total, Direct and Indrect Reductions } \\
\text { without additional EVs/PHEVs }\end{array}$ & & & & & & & 15 & 1184 & 15 & 427 \\
\hline & $\begin{array}{l}\text { including support for additional } \\
\text { EVs/PHEVs }\end{array}$ & & & & & & & 18 & 1322 & 18 & 509 \\
\hline
\end{tabular}


The final four columns show the corresponding potential to reduce the energy consumption and the $\mathrm{CO}_{2}$ emissions of the entire electricity sector. Columns eight and ten provide the absolute reductions and columns seven and nine provide the reductions as percentages for energy and $\mathrm{CO}_{2}$ emissions, respectively. That is, the absolute potential reduction divided by U.S. electricity sector total generation and $\mathrm{CO}_{2}$ emissions in 2030. This facilitates direct comparison of the magnitude of the reductions with the RPS that require certain fractions of electricity to be supplied by renewable generation (and, in some states, energy efficiency) in a decade or two.

The estimates assume deployment of smart grid technologies in $100 \%$ of utility service territories in the United States. To a first order, the estimates provided can be scaled downward linearly to reflect estimate impacts for less than $100 \%$ deployment, in proportion to the percentage of the U.S. population served by a smart grid.

Each mechanism is generally described with its estimated reduction in energy consumption and $\mathrm{CO}_{2}$ emissions in the discussion that follows. The full results of the literature review and analysis of each of the nine mechanisms is contained in Attachment 1, and the details of the calculation of the estimated reductions are contained in Attachment 2.

As might be expected, there is considerable variation in the potential of the mechanisms to reduce energy consumption and $\mathrm{CO}_{2}$ emissions. A primary purpose of this investigation is to provide some guidance as to which mechanisms are most important. Some mechanisms were estimated to have a negligible effect; in one case, no firm evidence could be identified to justify an estimate greater than zero. While none of the direct mechanisms is more than $3 \%$ individually, they combine to form a significant contribution toward the nation's carbon management goals for its electricity sector. The total direct reduction of $12 \%$ includes an estimated reduction of 3\% from eventually supplying an additional $9 \%$ of vehicle-miles traveled (VMTs) with electricity. The indirect reductions provide an additional $6 \%$, primarily from reinvesting savings from the operational costs of integrating a portfolio of $25 \%$ renewable generation. This is based on the cost of capacity to meet the intermittency characteristic of wind resources. The combined potential of the mechanisms (18\%) is substantial.

It must be noted that the range of uncertainties is high, often $50 \%$ or more of any given estimate. This is not unexpected given the exploratory nature of this analysis and the early stage of development of a smart grid. Narrowing the range of this uncertainty and refining the estimates is the focus of many of the recommendations for follow-on analysis, and is particularly important for the mechanisms that offer the most significant potential. While any given mechanism has some likelihood of providing little or no savings, the probability that this would be true for all the mechanisms is much less, in light of the wide variety of how the savings are achieved. A smart grid, although not the central means of providing the savings that energy efficiency and renewables represent, nevertheless appears to have a significant role in enhancing those savings and achieving them at less cost.

\subsection{The Smart Grid and Energy Efficiency}

Utilities increasingly consider energy efficiency as a "fifth fuel" because of concern about the implications of a carbon-constrained world for their business and their obligation to serve customer demand. One driver is that many utilities are having difficulty gaining approval for new base-load generation (generally coal-fired power plants) from state regulators because of projected carbon 
restrictions. Among many recent examples is Duke Power's commitment to purchase carbon offsets to cover the difference in carbon emissions from its proposed new coal-fired power plant as compared to a cleaner combined-cycle natural gas turbine and also retire two older dirty coal-fired power plants. This increases the attractiveness of energy efficiency, because natural gas tends to be more expensive than coal and increasingly relies on imports with consequent price volatility.

A second driver is that many states have passed or are considering passage of an RPS that sets a minimum requirement for renewable generation and, in many cases, includes energy efficiency as a means of meeting the RPS or as a separate requirement. A third driver is the probability that national capand-trade legislation for carbon emissions will be passed, which in effect raises electricity prices and hence makes efficiency more attractive. It may also be accompanied by a national RPS. A fourth driver is that state regulators are advancing policies to remove utility and customer disincentives to greater penetrations of energy efficiency. Related to these drivers is a growing recognition of the need to address key barriers to obtaining the large potential of the energy efficiency resource (EPA 2008; EPRI 2009; McKinsey \& Company 2009).

If the increased role of renewables and energy efficiency comes to pass, as seems increasingly likely, then the United States will need to make drastic changes in everything from its vehicles, buildings, and the way in which it generates electric power. One way to think about how this will affect utilities is introduced in Figure 3.1, which shows a well-known carbon supply curve (McKinsey \& Company 2007). It shows the technical potentials of various carbon reduction technologies in the United States (the width of each bar) against their equivalent cost per ton of avoided $\mathrm{CO}_{2}$ (the height of each bar), ordered from the lowest to the highest cost.

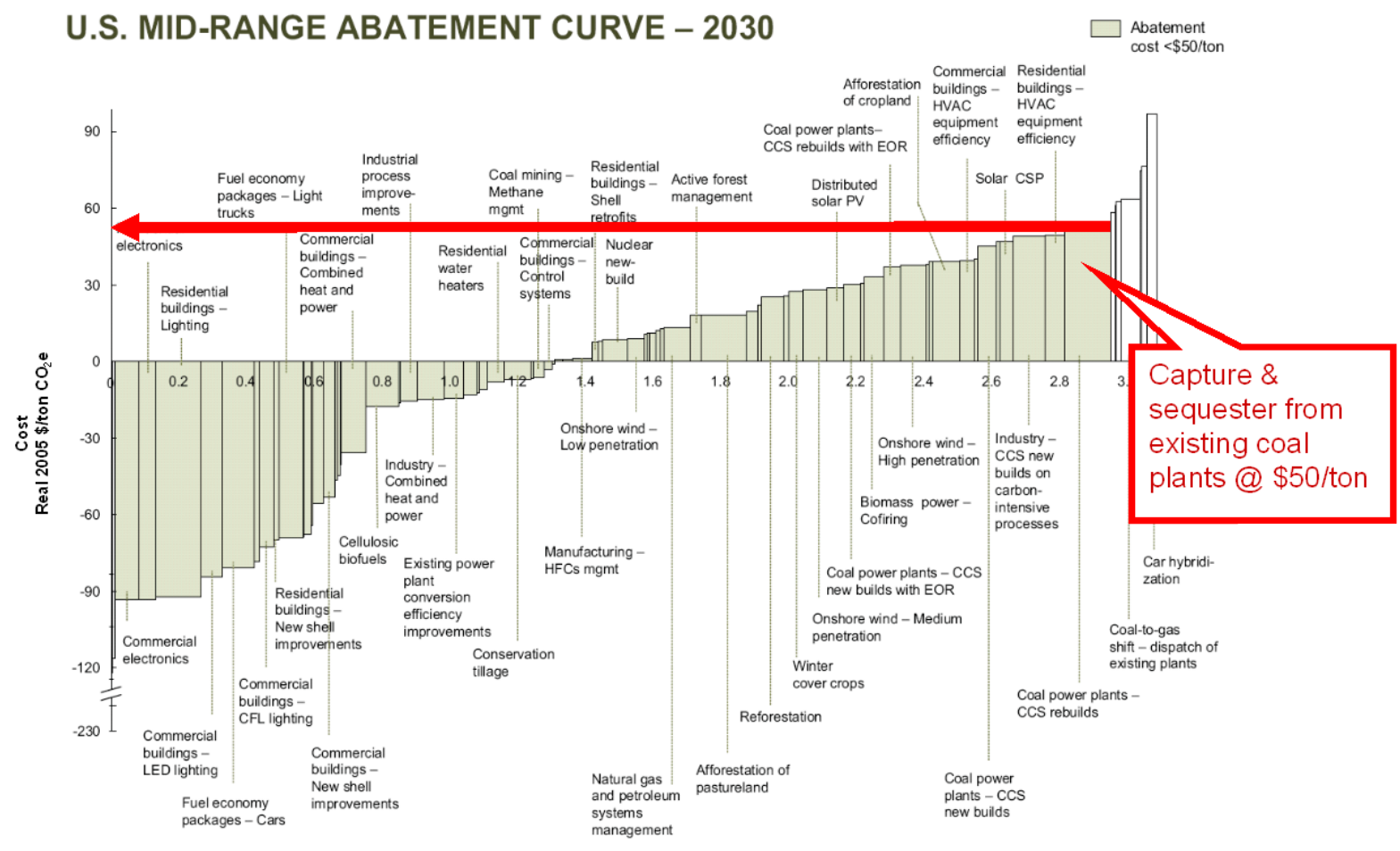

Source: McKinsey analysis

Figure 3.1. Carbon Cost Abatement Curve. Source: McKinsey \& Company (2007) 
It is striking to note that nearly one half the $\mathrm{CO}_{2}$ reductions, mostly from efficiency measures, could be achieved at negative cost. That is, the energy savings alone more than pay for the cost of the efficiency measure over its lifetime. This illustrates one of the key barriers: energy efficiency investments, largely left to the consumer, lags considerably below those that are cost effective. In economic terms, consumers apparently discount efficiency investments or, equivalently, have very short payback requirements.

If carbon capture and sequestration from coal-fired power plants becomes a viable option in the future, it has been suggested that it may cost approximately $\$ 50 /$ ton $\mathrm{CO}_{2}$. Assuming there is no shortage and ready access to sequestration sites, this may become the "limiting option" and effectively places a cap on carbon prices. This translates to a doubling of wholesale power costs from coal (currently approximately $5 \phi / \mathrm{kWh}$ ), which would render all the carbon reduction measures in Figure 3.1 to the left of carbon sequestration (the shaded "bars") as cost effective.

This suggests that massive energy efficiency and renewables programs are likely in the future, at a scale beyond what was generally imagined just a few years ago. Utilities have both the motive and the means to deploy energy efficiency on a massive scale, because they have good access to capital at more attractive rates than consumers, and are in the business of making large infrastructure investments that earn steady, but modest long-term rates of return. That they can gain carbon credits and meet RPS requirements through energy efficiency investments further increases their motivation. Properly incentivized, accelerated deployment of utility-funded energy efficiency programs could have a major role in reducing $\mathrm{CO}_{2}$ emissions.

It is against this backdrop that we examine the role of a smart grid. Although none of the costeffective carbon reductions from improved energy efficiency in Figure 3.1 explicitly require a smart grid, a smart grid may facilitate deployment of efficiency measures. This is particularly true for some of those in the middle and second half of the curve that are marginally cost effective today or will require a price on carbon to become cost effective. Several of these are the subject of subsequent discussions of the mechanisms in this report. The following subsections summarize the mechanisms that relate to energy efficiency.

\subsubsection{Conservation Effect of Consumer Information and Feedback Systems}

Many demand response projects have reported some customer energy savings, typically a few percentage points, in addition to their primary objective of reducing peak loads. While some energy savings can be attributed to physical effects of reducing load during peak load times, the primary basis for the savings is likely to be the effect of feedback provided to consumers on their usage patterns as part of these programs. This mechanism is summarized here, with detail on the literature review and conclusions provided in Attachment 1.

It is worth noting that demand response is fundamentally a curtailment behavior, and so has more in common with energy conservation than energy efficiency. Although sometimes used to indicate both, energy efficiency more properly describes obtaining the same service or amenity from a device for less energy input (i.e., a more efficient lighting source or air conditioner), whereas energy conservation means doing without the device's service or amenity. In this respect demand response and conservation are similar, although they occur over different time scales. Demand response for managing peak loads involves, at most, 100 or so hours a year, and seldom more than a few consecutive hours. Demand 
response used to provide ancillary services involves time periods of a few minutes. Any associated loss of amenity is occasional and short term, and in some cases negligible. When the objective is to save energy, conservation must affect a large fraction of the hours the device is used, and the loss of amenity is more or less continual.

Demand response itself can reduce energy consumption because controlling an end-use to lower peak load demand shifts the load to other times, or in some cases actually eliminates some consumption. A prime example of the latter is lighting - dimming lights on peak load also saves energy. Other mechanisms are more subtle, second-order effects. For example, deferring air-conditioning loads until later in the evening allows the air conditioner to run when it is cooler outside, hence, when it operates more efficiently. Counteracting this effect, control strategies that pre-heat or pre-cool in advance of peak load demand periods can result in slightly higher overall energy use. Controls that cycle water heaters off effectively reduce the water temperature somewhat, and can produce substantial savings if hot water is used during those times. ${ }^{1}$

Most of the large end uses, aside from lighting and electronics, are fundamentally controlled by a thermostat (heating, air conditioning, water heating, refrigeration, and drying). So, to a first order, deferring energy input into a device simply results in an equal amount of energy input later to heat the device back up or cool it back down to its original temperature. It is this eventual restoration of service that distinguishes demand response from conservation, and the reason the latter can produce large energy savings when the former typically does not.

Although there may be some physical explanation for the energy savings reported by demand response programs, we believe the primary contribution comes from heightened awareness of energy use on the part of the participants. This awareness can come simply from the decision to participate, but demand response programs usually offer formal feedback mechanisms to the consumer, based on the AMI interval consumption data that shows patterns of usage over the day and week. In some cases, these feedback mechanisms are supplemented by web-based portals or in-home displays that deliver the information and may include a breakdown of consumption by end use.

The focus of our analysis of this mechanism is to determine the potential benefits of leveraging these smart grid assets to provide detailed and timely energy feedback and a variety of usage information. Fundamentally, the objective of feedback is to overcome the issue of energy invisibility, which refers to the gradual de-coupling of overt human behavior from energy use, reflected by the historical transition from chopping wood for fuel, to shoveling coal for a furnace, to gas and electric power delivered seamlessly and automatically on demand.

To do this, we examined the results from a wide range of studies of feedback mechanisms on consumers (primarily residential). The studies reviewed provide convincing evidence that consumers will

\footnotetext{
${ }^{1}$ Peak load demand reductions can be obtained from either energy efficiency or conservation measures. The peak load reductions are larger for measures that reduce consumption for an end use that tends to be higher during peak load periods (hot summer days in most of the United States), like air conditioning and commercial lighting. Thus, energy efficiency and conservation can make a valuable contribution to the same objective (managing peak load demand) and, hence, compete with smart grid assets like demand response. Unlike peak load demand, however, they have a significant negative impact on utility revenues and may require regulatory action to motivate utilities to make increased use of them. They do not require the communications or the coordinated control that characterize smart grid assets, so the peak load effect of energy efficiency and conservation is not a subject of this analysis.
} 
change their energy consumption behavior in response to feedback, and that the conditions surrounding feedback, such as frequency and specificity, are influential variables. This implies that a smart grid/metering system may yield considerable savings in terms of end-use conservation, with a basic goal of time-of-use load shifting. Feedback tends to be most effective when it:

- is based on actual usage data

- is provided on a frequent basis (daily is better than weekly, etc.)

- involves goal setting and choice

- is provided over a year or more

- involves specific behavioral recommendations regarding appliances

- involves normative or historical comparisons.

Fischer (2008) contends that these items favor the smart grid capabilities offered by AMI and twoway communication networks, which provide an effective way of engaging the consumer and providing tailored feedback.

The energy-use reductions achieved from a range of projects examined by Fischer (2008) range from $5 \%$ to $20 \%$, with a median of approximately $6 \%$. Similar results have been observed in utility field studies reviewed by Faruqui et al. (2009). We have adopted this estimate of the potential. The key issue surrounding these results is whether they are sustainable over a time period of years and decades. Given that consumers generally volunteer for such studies, there may be some built-in bias up front. More importantly, does the consumer continue to seek out feedback, or internalize it and translate that into permanent changes in behavior?

To be conservative, we have shifted the range of uncertainty lower, to a low of $1 \%$ and a high of $10 \%$. We assume that, with respect to the contribution of a smart grid, a direct reduction of $6 \%$ in electricity consumption only for the residential and small/medium commercial building sectors. At a minimum, such a feedback mechanism requires an interval meter that is fundamental to a smart grid. Large commercial buildings and industrial customers generally already have such meters, and consume enough energy to install relatively sophisticated feedback systems and pay a staff member to monitor energy use, so it is difficult to assert that a smart grid is essential to achieving similar savings in this customer class.

Quite substantial potential reductions of 3\% in electricity consumption and associated $\mathrm{CO}_{2}$ emissions are estimated in Table 3.3, with the calculations documented in Attachment 2. No indirect reductions from capital or cost savings are expected.

Table 3.3. Estimated Direct Impacts of the Conservation Effect of Consumer Information and Feedback Systems

\begin{tabular}{|c|c|c|c|c|c|c|c|c|}
\hline \multirow[b]{3}{*}{$\begin{array}{c}\text { Est. } \\
\%\end{array}$} & \multicolumn{4}{|c|}{ Reduced Energy Consumption (2030) } & \multicolumn{4}{|c|}{ Electric Sector Annual Reductions (2030) } \\
\hline & \multirow[b]{2}{*}{$\begin{array}{c}\text { Low } \\
\%\end{array}$} & \multirow[b]{2}{*}{$\begin{array}{c}\text { High } \\
\%\end{array}$} & \multicolumn{2}{|c|}{ Baseline Electricity Consumption } & \multicolumn{2}{|c|}{ Energy } & \multicolumn{2}{|c|}{ Carbon Emissions } \\
\hline & & & End-Use Sector(s) & $\left(10^{9} \mathrm{kWh} /\right.$ year $)$ & $\begin{array}{c}\% \text { of } \\
\text { United } \\
\text { States }\end{array}$ & $\begin{array}{c}\left(10^{9} \mathrm{kWh} /\right. \\
\text { year })\end{array}$ & $\begin{array}{c}\% \text { of } \\
\text { United } \\
\text { States }\end{array}$ & $\begin{array}{c}\text { (MMT/ } \\
\text { yearr) }\end{array}$ \\
\hline 6 & 1 & 10 & Residential & 1722 & 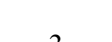 & 15 & 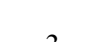 & 0 \\
\hline 6 & 1 & 10 & Small/Medium Commercial Buildings & 854 & 3 & 153 & 3 & 92 \\
\hline
\end{tabular}


Two-way communications like that provided by a smart grid AMI network is also required if the feedback information is centrally processed and delivered in near real time; billing inserts do not provide the timelines or frequency characteristic of effective feedback. Whether a web-based billing information portal is sufficiently engaging over the long run remains to be proven. The expense of a dedicated home energy display would not be required if the information can be effectively delivered using the internet. Google is already offering such a capability on their user-specific home page, when granted access to the data from the meter.

Currently existing software tools can estimate a customer's appliance and equipment usage based on population average values and offering generic guidance on saving energy. More specific and effective feedback and advice can be provided to the consumer if the demand response control (e.g., a thermostat) is used to provide on/off status information for the device it controls. Even in a fully deployed smart grid, we do not anticipate that virtually every customer will participate in demand response programs.

However, we do anticipate that, participation in smart grid deployments by 2030 will be high enough that smart thermostats and smart appliances will be widespread, and they can provide the basis for end-use breakdowns.

The breakdown process can be conducted either centrally at the utility, or within the home or business. The advantage of the latter is that it strictly maintains the privacy of the customer. The disadvantages are that it requires additional processing power and software installation in a home or building energy-management system and it cannot offer comparisons with the energy consumption of peer groups.

\subsubsection{Joint Marketing of Energy Efficiency and Demand Response Programs}

Joint marketing of energy efficiency and demand response programs would provide customers a unified vision that connects utility programs and education materials. This mechanism would capture the synergy between energy efficiency and demand response programs, saving costs in administering, advertising, educating, and recruiting, to make each more cost effective and impactful. The coordination would also enable the most cost- and/or $\mathrm{CO}_{2}$-effective solution to be implemented by consumers. This mechanism is summarized here, with detail on the literature review and conclusions provided in Attachment 1.

Electricity customers want to be presented with a unified vision of how they can change their electricity consumption to save money and help the environment, without the technical terminology that asks them to distinguish between energy efficiency and demand response programs. This strongly suggests that consumers should be provided "one-stop shopping" when connecting to utility programs and consumer education materials. The strong synergy between energy efficiency and demand response programs can also be exploited to save advertising, educating, recruiting, measurement, and evaluation costs. In addition, energy efficiency measures generally also produce peak load reductions and associated costs, which should be accounted and incentivized as peak load savings. 
This indirect mechanism would combine the administration of energy efficiency and demand response ${ }^{1}$ programs to achieve cost savings that could be reinvested in efficiency programs. However, the literature review and contact with experts in the energy efficiency and demand response fields did not reveal any program data or information that could be used to estimate administrative cost reductions or increases in program effectiveness. Based on this finding, indirect reductions in electricity and associated $\mathrm{CO}_{2}$ emissions shown in Table 3.4 and calculated in Attachment 2 are estimated to be zero, as no basis could be estimated for determining administrative cost reductions or increases in program effectiveness that might ensue. No direct reductions are expected.

Table 3.4. Estimated Indirect Impacts of Jointly Marketing Energy Efficiency and Demand Response Programs

\begin{tabular}{|c|c|c|c|c|c|c|c|c|c|}
\hline \multirow[b]{3}{*}{$\begin{array}{c}\text { Est. } \\
\%\end{array}$} & \multicolumn{5}{|c|}{ Avoided Expenditure Reinvested to Save Carbon (2030) } & \multicolumn{4}{|c|}{ Electric Sector Annual Reductions (2030) } \\
\hline & \multirow[b]{2}{*}{$\begin{array}{c}\text { Low } \\
\%\end{array}$} & \multirow[b]{2}{*}{$\begin{array}{c}\text { High } \\
\%\end{array}$} & \multicolumn{2}{|l|}{ Baseline Captial Expenditure } & \multirow[b]{2}{*}{$\begin{array}{c}\text { Savings } \\
\left(10^{9} \$\right)\end{array}$} & \multicolumn{2}{|c|}{ Energy } & \multicolumn{2}{|c|}{ Carbon Emissions } \\
\hline & & & Investment & $\left(10^{9} \$\right)$ & & $\begin{array}{l}\% \text { of } \\
\text { United } \\
\text { States }\end{array}$ & $\begin{array}{c}\left(10^{9} \mathrm{kWh} /\right. \\
\text { year })\end{array}$ & $\begin{array}{l}\% \text { of } \\
\text { United } \\
\text { States }\end{array}$ & $\begin{array}{c}\text { (MMT/ } \\
\text { year) }\end{array}$ \\
\hline 0 & 0 & 1 & $\begin{array}{l}10 \% \text { Demand Response, Residential } \\
\text { @ } \$ 400 / \mathrm{kW} \& 8.8 \phi / \mathrm{kWh}\end{array}$ & 15 & 0.0 & & & & \\
\hline 0 & 0 & 1 & $\begin{array}{l}\text { 10\% Demand Response, } \\
\text { Small/Medium Commercial } \\
\text { Buildings @ } \$ 300 / \mathrm{kW} \& 8.8 \phi / \mathrm{kWh}\end{array}$ & 6 & 0.0 & 0 & 0 & 0 & 0 \\
\hline
\end{tabular}

However, to place the potential of this mechanisms in perspective, if combining the marketing and outreach of energy efficiency and demand response programs resulted in a $1 \%$ savings in program operating costs and the savings were re-invested in energy efficiency, a reduction in electricity supply of $3 \mathrm{~B} \mathrm{kWh}(0.05 \%)$ and associated $\mathrm{CO}_{2}$ emissions of $1.4 \mathrm{MMT}(0.04 \%)$ might be expected. This is based on the combined potential for the residential and small/medium commercial building sectors. Because of the complexity of energy-using systems in large commercial and industrial customers, we anticipate that both energy efficiency and demand response programs will need to be delivered with a customer-specific focus in which a smart grid may play only a small role.

Further investigation of the potential for jointly marketing energy efficiency and demand response programs is suggested.

\subsubsection{Key Enabling Technology: Disaggregation of Total Loads into End Uses}

This section describes how the measurement and communication capabilities of a smart grid can be leveraged to provide unprecedented detail on customer end-use consumption. While the ability to disaggregate loads does not provide direct or indirect reductions, it forms the technical basis for providing remote diagnostics for HVAC loads via a smart grid, as discussed in Section 3.1.4 (Mechanism C), and for improved $M \& V$ of energy savings from efficiency measures, as described in Section 3.1.5

\footnotetext{
${ }^{1}$ Demand response is most closely associated with curtailment behavior, as distinct from energy efficiency measures and behavior. While often achieving similar goals, demand response is principally designed to reduce peak/critical loads through load shifting and may not provide direct reduction in energy use, whereas energy efficiency is designed to reduce overall energy consumption and provide long-term savings. Both are intended to provide monetary savings to consumers, but energy efficiency provides virtually no change in consumer comfort and usability, whereas demand response may have a short-term impact on consumer comfort and/or service.
} 
(Mechanism D). It can also be used to "mine" for customer-specific energy efficiency and demand response opportunities that have been overlooked, and as the basis for enhancing feedback to consumers on their individual appliances and end uses, as discussed in Section 3.1.1 (Mechanism A). Thus, the addition of relatively straightforward automated analytic processes coupled with smart grid measurement and communication capabilities forms a key enabler for obtaining energy and carbon benefits.

The following four figures help show the improved end-use resolution that could be provided by smart grid technology. Figure 3.2 shows the monthly energy consumption profile for a residence from data obtained by a standard utility meter. The home is located in a hot desert climate with high summer peak load demand due to dozens of days in which the outside temperature exceeds $100^{\circ} \mathrm{F}$. With a monthly resolution, the energy consumption is actually at its lowest in the summer, making it impossible to discern much about the end-use consumption of the home without the use of an engineering model and a lot of assumptions that introduce considerable uncertainty.

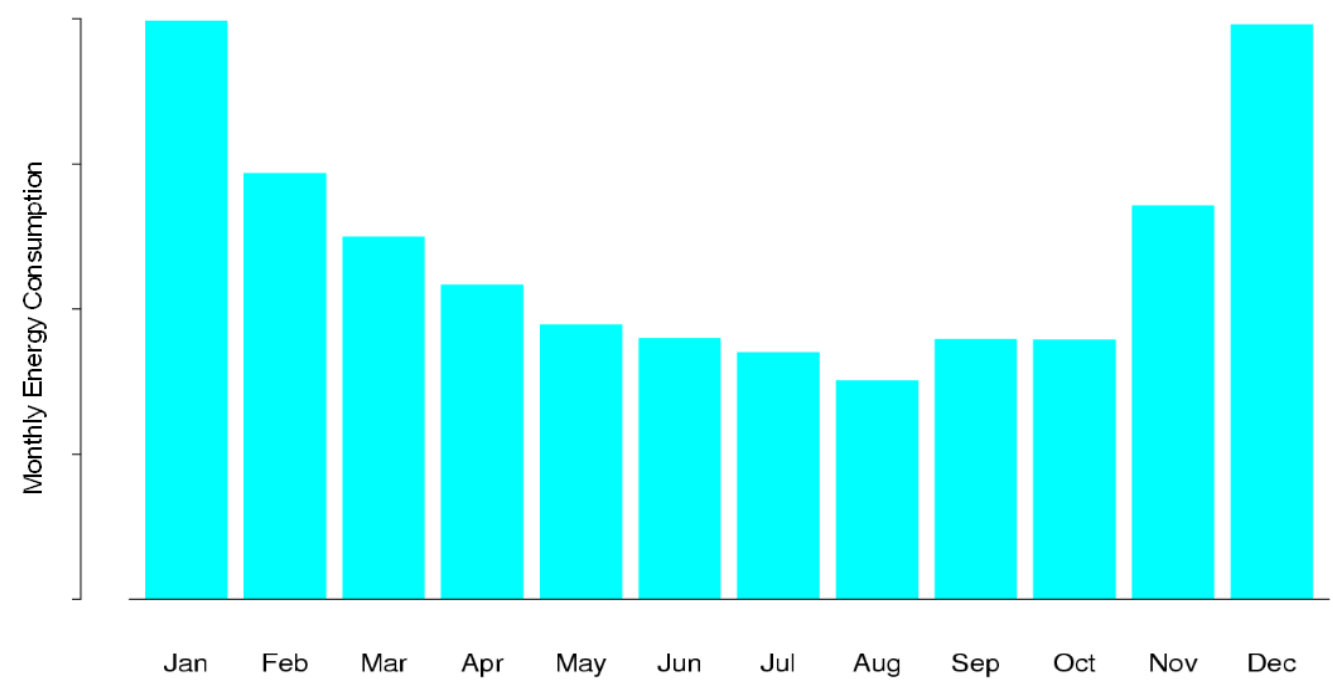

Figure 3.2. Monthly Energy Bills from Typical Home in a Hot Desert Climate

Figure 3.3 shows a profile of the same monthly energy bills plotted against the average monthly temperature. This is the technical basis for a well-known standard technique for analyzing monthly billing data, originally called the Princeton Scorekeeping Method (PRISM; Fels and Reynolds 1993). The PRISM method attempts to find the best statistical fit for three lines describing the heating, cooling, and base load (all other loads) for a building. The base load is estimated from the minimum monthly bills, assuming that it is relatively constant. Thus, the estimate of the base load is a horizontal line, as shown in Figure 3.3.

When the home is heated by electricity, the monthly load is expected to increase as the outdoor temperature decreases. The physics of heat flow suggests this relationship is linear with temperature, although when aggregated to the monthly level, there tends to be somewhat of an upward bend to this curve. The deviations in the monthly winter consumption data from the linear assumption in Figure 3.3 are illustrative of this effect. If the heating is provided by a heat pump, this curvature is greatly 
exaggerated, because it is much less efficient at supplying heat when it is colder. The scarcity of the monthly data and the effect of the curvature make it difficult to fit these lines with a high degree of confidence.

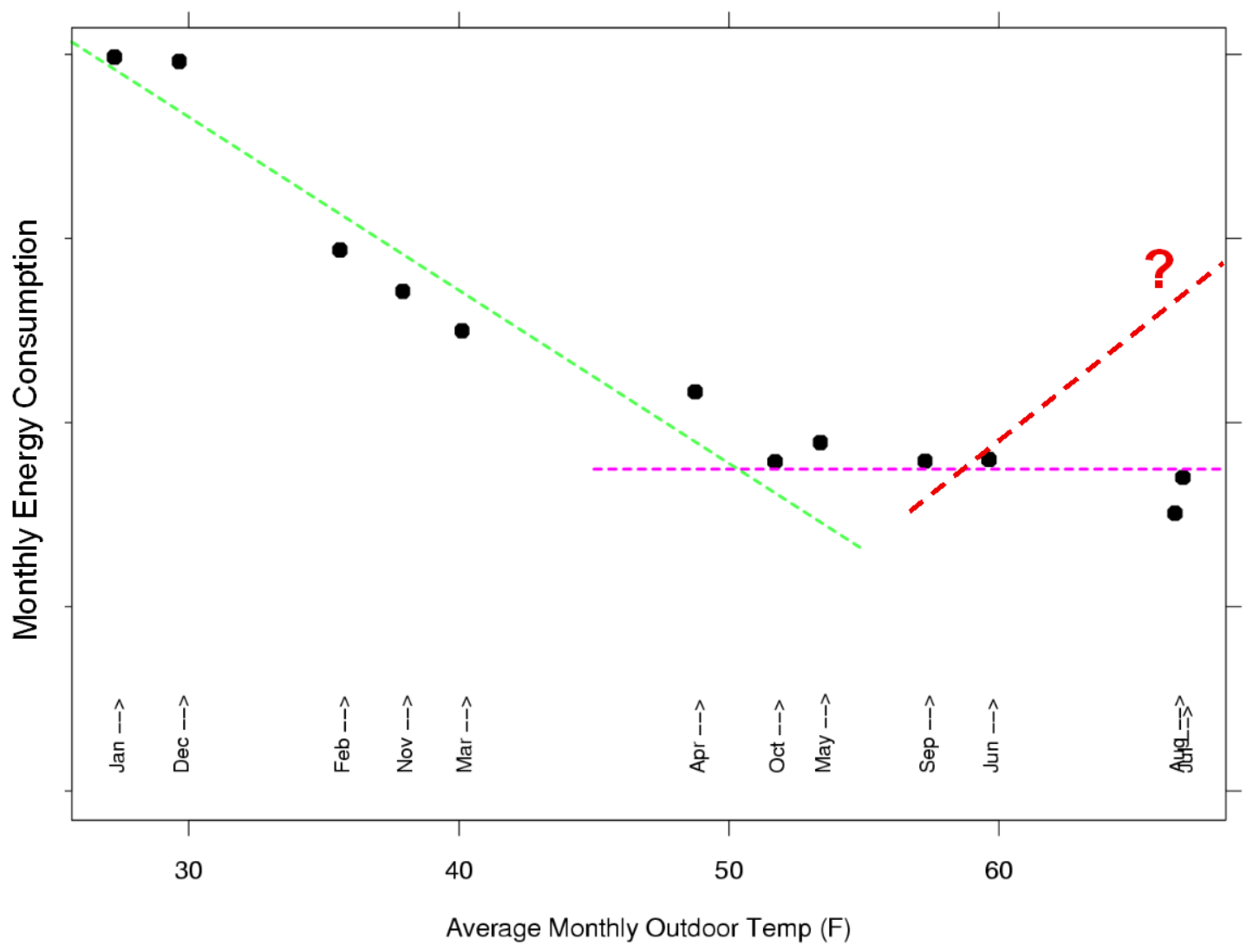

Figure 3.3. Monthly Energy Bills from Typical Home vs. Monthly Average Temperature

Trying to estimate the air-conditioning loads in this home using monthly data is impossible. The relationship between consumption and temperature in the summertime is non-existent and estimating the end-use consumption is highly uncertain, particularly the expected air-conditioning load. It is not unreasonable to make the interpretation that there is no air-conditioning load in this home-in fact, this is far from the case.

PRISM-type methods were developed for the purpose of energy efficiency program evaluations involving hundreds or thousands of buildings, rather than providing building-specific analyses. Despite their limitations, they are suitable for this purpose as long as any errors introduced by the limitations of the method are random from one building to the next. They are considerably less well suited to detailed analysis of individual buildings, as illustrated here.

Figure 3.4 shows the increased information content that can be provided by leveraging smart grid assets. First, thousands of hourly intervals of consumption from a smart meter provide a much stronger statistical basis for fitting a line or a curve to the data. Second, examining the results separately for each 
hour of the day provides much additional clarity. Third, with the additional use of the on/off signal that could be provided by a smart thermostat, the total consumption provided by a smart meter can be disaggregated into subtotals for heating, cooling, and "other" base loads with reasonable precision.

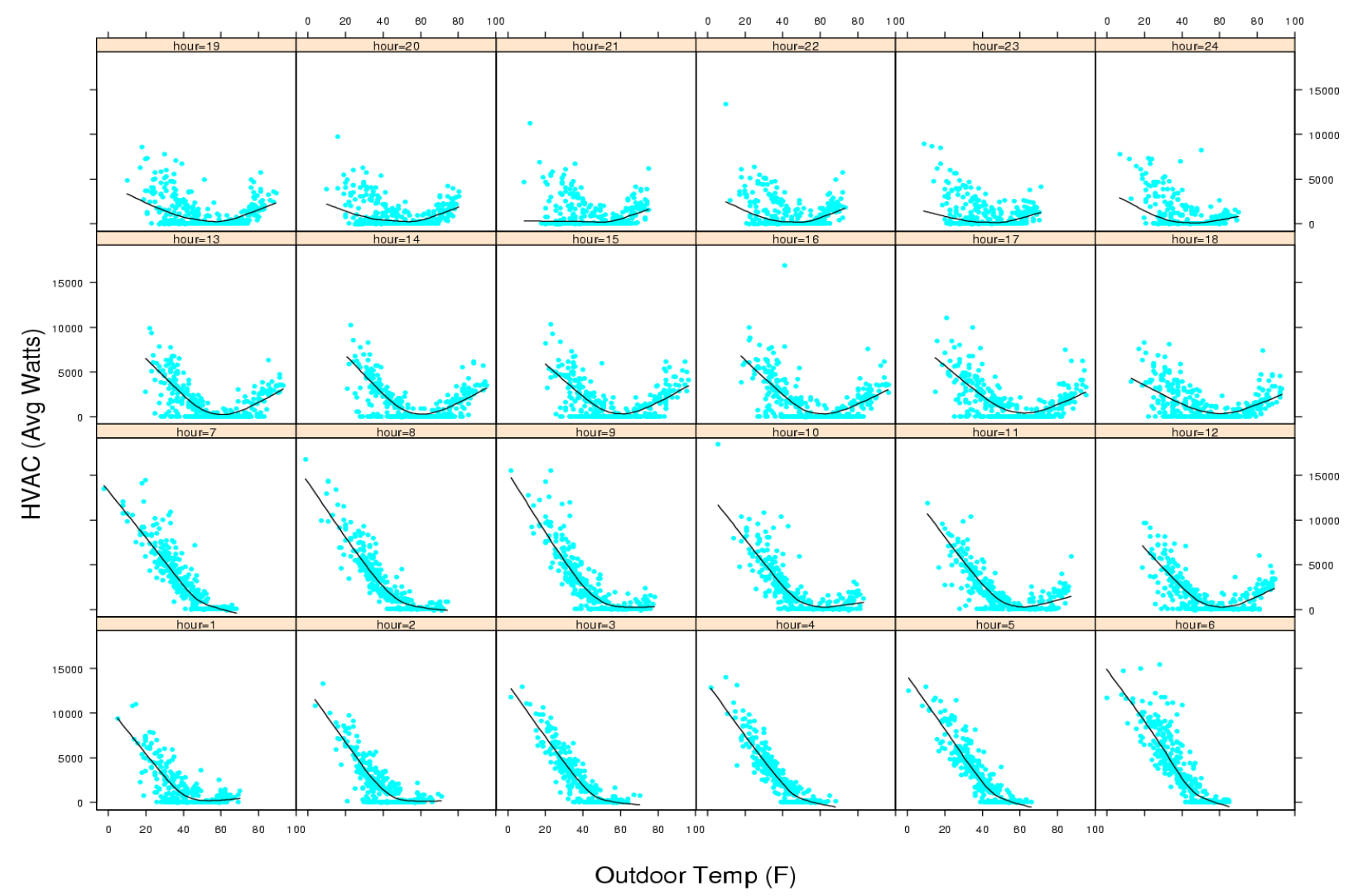

Figure 3.4. Hourly Load Data vs. Temperature, One Plot for Each Hour

This process is entirely analogous to what has been termed non-intrusive load monitoring (NILM; Drenker and Kader 1999). NILM uses short interval readings from a meter and uses a cluster analysis to look for common changes in the level of power consumption. These can then be mapped to a specific major appliance, e.g., a space heater, air conditioner, or water heater, either by 1) rules of thumb, if the consumption level is relatively standard, as for water heaters, or 2) manually activating major appliances and denoting the cluster into which the resulting load is mapped.

The same basic process can be applied to a smart-grid-based system by using the on/off status signal from the thermostat instead of the cluster analysis. This provides an unequivocal signal with which to flag on/off events. More importantly, for mass deployments in which the process must be completely automated, it inherently associates the changed consumption level to a specific appliance or load, eliminating the need for the manual tests. Integrating the resulting on/off events and power levels over time produces a good breakdown of the end-use composition of the total load collected by a smart meter.

Figure 3.4 provides separate plots of the HVAC energy consumption for each hour of the day as a function of the outside temperature for that hour. The air-conditioning load becomes readily apparent with this more granular data. In hours 1 a.m. through 10 a.m. (hours 1 through 10), there is no apparent 
cooling load. From 11 a.m. through 10 p.m. (hours 11 through 22), the right side of the distribution increases markedly, showing the air-conditioning load with increased clarity.

As a final step, models can be fit to each of the disaggregated end-use subtotals. Figure 3.5 shows non-linear, non-parametric fits (a lowess curve) for each of three end-use load subtotals for hour 15 (3 p.m.). These are stacked, i.e., the top curve represents the total load. The end-use load is the distance between the curves (or the x-axis).

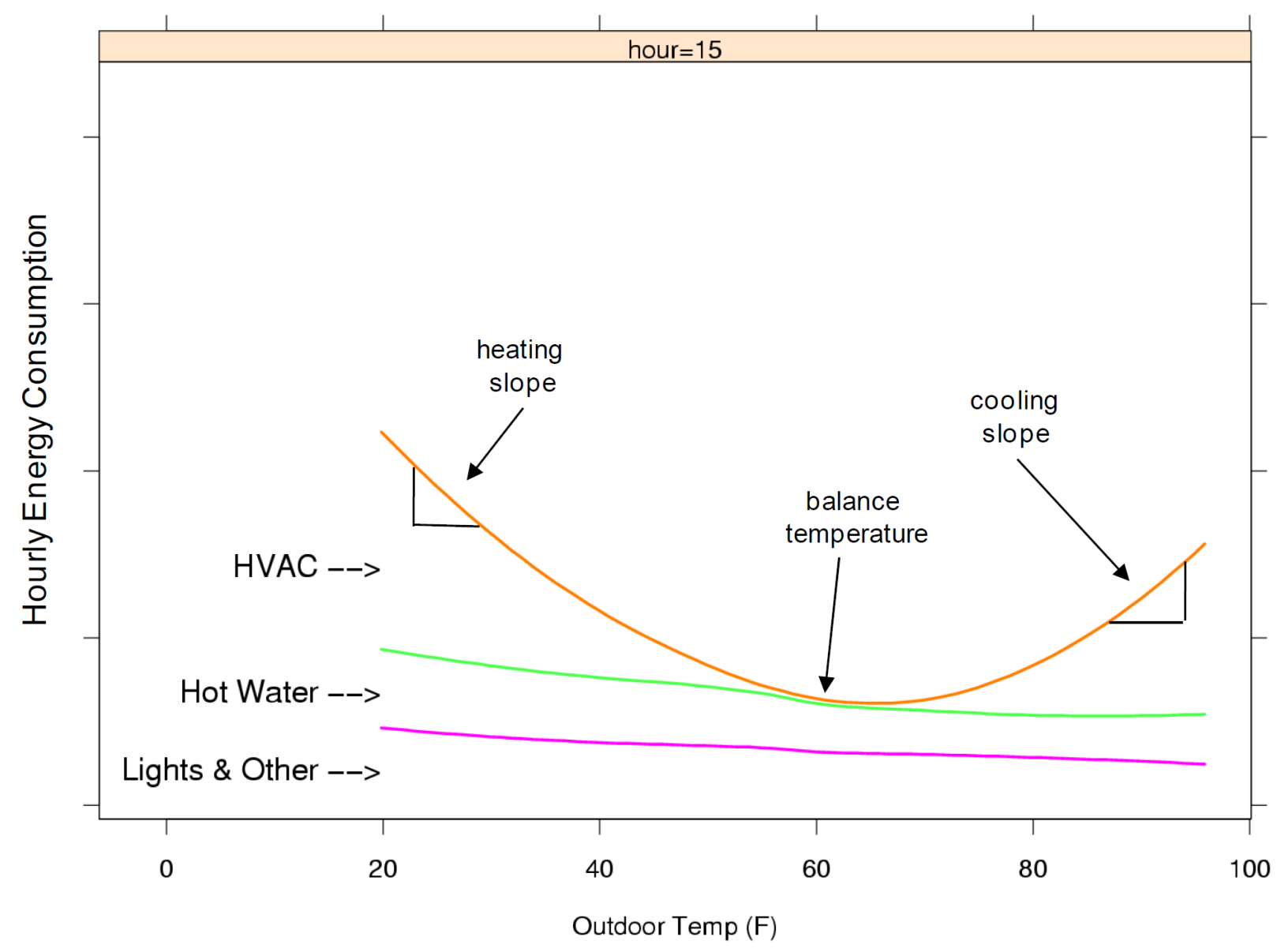

Figure 3.5. Non-Linear Models of Three End-Use Load Subtotals for Hour 15 (3 p.m.)

Such models provide several additional types of information. First, the subtotals themselves form a much finer end-use resolution with which to spot changes in consumption for smaller appliances and loads from lights and electronics. Second, it should be noted that the consumption for water heating and for lighting plus "other" loads (the rest of the appliances in the home) are not constant throughout the year, but instead have a pronounced linear trend increasing with colder outside temperatures and winter months. This is typical of U.S. residences that have been metered at the end-use level, in which virtually all non-HVAC end uses increase $10 \%$ to $20 \%$ or more from summer to winter, on average (Pratt et al. 1993). Thus, the assumption of PRISM-like methods-that the base load is a constant-tends to overstate heating and understate cooling loads. 
Finally, the shape of the HVAC model provides important clues about the home's thermal performance upon which to base further analysis. The balance temperature - the outside temperature at which the home needs neither heating or cooling because of heat gains from appliances and the sun-is noted as approximately $60^{\circ} \mathrm{F}$. Thermal physics suggest the balance temperature is the ratio of these heat gains to the envelope heat coefficient (the heat loss per degree $\mathrm{F}$ indoor-outside temperature difference). The slope of the heating and cooling parts of the curve indicate the ratio of the heat loss coefficient and the heating or cooling system efficiency, respectively.

The information that can be derived from a smart grid's infrastructure can provide deeper and much more valuable insights into the performance of individual buildings and populations of customers than simple analysis of monthly bills. With the advent of AMI, near-real-time communication systems, and the advanced data management and demand response control strategies, a smart grid may be capable of:

- providing simple diagnostics of energy systems to provide early detection of problems

- supporting high-fidelity M\&V of savings from energy efficiency programs

- ensuring the persistence of savings from energy efficiency over time

- data mining to identify customers with significant energy efficiency opportunities

- providing detailed feedback to customers on how to reduce their energy costs and carbon footprint

- analyzing the effects of behavior on energy consumption for populations of customers

- attributing carbon credits to utilities or customers, as appropriate.

The first two bullets are mechanisms analyzed in the next two sub-sections of this report.

It is important to understand what, if any, the marginal costs for deploying a smart grid capable of delivering these benefits are. The cost of AMI and associated communication systems are justified by other services preformed, and near-universal deployment of AMI in a smart grid is generally a given. The time resolution provided can bring about some of these potential benefits.

The additional insight provided by the disaggregation of the total load into end uses in homes and small commercial buildings is dependent upon the deployment of smart thermostats, at a minimum. These thermostats need to be capable of providing on/off status for heating and cooling back to a home or building's local area network, or the AMI meter itself. Such capabilities are available today and by 2030 we assume such thermostats will be nearly universal, since enough consumers will be participating in demand response programs to drive marginal hardware costs down to negligible levels. This assumes that direct load-control approaches, which do not require a thermostat but simply interrupt power to a device, are not the predominant form of demand response. This assumption is based on industry trends to more consumer-friendly approaches using thermostats, and the diminishing differential in cost between the two approaches.

Electric water heaters are likely to be similarly equipped (with load-control devices capable of reporting on/off status) in parts of the country, because they are important targets for demand response today, and involve little perceptible sacrifice in amenity. Smart appliances are the focus of an intense development effort on the part of manufacturers today. If utility programs provide incentives such as 
rebate programs, or if such features are required by standards, then smart appliances may similarly become nearly universal elements of a smart grid within the coming 20 years.

If the disaggregation process takes place "inside the meter," then a suitable processor to host the analysis of the signals is required, and the results could be delivered through an AMI communications network without extending its capabilities. This simple analysis can be a background process on a home computer, built into cable television systems, hosted by a home energy display platform, or conducted within the meter itself, for example.

If the disaggregation process takes place at the utility, then the meter must integrate and store the additional on/off signals. This type of modest improvement in AMI meter capabilities is already being contemplated in third-generation designs. An alternative is for the AMI communication network to have enough bandwidth to send the data in real time. This may come to pass for other reasons as smart grid assets are used for more sophisticated, real-time purposes, such as providing ancillary services (which will be significantly increased by renewable wind and solar generation, as discussed in Sections 3.2.1 and 3.2.2).

The cost of AMI and communication systems are justified by other services performed, which leaves the cost of measurement technology and central analysis functions to be covered. Of these two functions, it is likely in many cases that the cost of the measurement capability will be included in the technology as part of the demand response, and therefore justified on that basis.

Thus, it is certainly possible that all the capabilities needed to enable these benefits will be present in a smart grid in the future. It is doubtful, however, that the potential benefits for energy, alone (the bulleted list) above will be sufficient to pay for them. What is important is that these potential "sidebenefits" for enhanced capabilities be taken into consideration when designing a smart grid. This would leave the software that conducts the analysis and display functions as the primary cost. As it is for most software products, spreading this cost over large numbers of customers is the key to keeping costs down.

\subsubsection{Deployment of Diagnostics in Residential and Small/Medium Commercial Buildings}

This topic examines the potential reductions in energy consumption and carbon emissions that can be obtained from the provision of energy system diagnostics enabled by a smart grid to optimize energy use and reduce operating costs for energy and maintenance. A smart grid's real-time sensing and communication assets coupled with end-use information enable automated profiling of systems to detect malfunctions and alert the consumer immediately. In addition to detecting malfunctions, improvements in operation can be identified, such as verifying the operation of night setback of thermostats or identifying abnormal lighting and plug loads. This mechanism is summarized here, with detail on the literature review and conclusions provided in Attachment 1.

The technical basis for using smart grid assets to break down total energy use into end-use subtotals is discussed in Section 3.1.3. Here we focus on the use of this information to provide diagnostic services in residential and small/medium commercial buildings (commercial buildings less than $50,000 \mathrm{ft}^{2}$ in floor area), primarily for HVAC systems where the most significant energy-wasting failures occur. A smart grid's communications and sensing enable automated profiling of these systems to detect such 
malfunctions and alert consumers immediately. This is feasible because the HVAC systems in these classes of buildings are reasonably simple, served by unitary, single-zone equipment and thermostats, and they're uniform in design and operation.

A property of the refrigerant cycle in heat pumps and air conditioners is that their output and efficiency tend to drop together, while their input remains relatively constant. Thus, using the slope of the cooling curve (see Figure 3.5) and the run-times established by thermostat on/off status signals, declining efficiency could be detected long before complete failure of the equipment makes it obvious. Heat pumps that are providing inordinate amounts of heat with their auxiliary electric resistance backup are a similarly important target.

Another diagnostic check would be on the economizer function of commercial building ventilation systems. The economizer enables the building to supply $100 \%$ air from outdoors to obtain "free cooling" when air conditioning is required and the outside air is cool and dry enough. Economizers can save large amounts of energy in commercial buildings - when they work properly. Economizers are notorious for failing because the moveable air dampers tend to get stuck if not properly maintained. When not working properly they do not provide the savings and can even waste additional energy by remaining in the open $(100 \%)$ position all the time. The "hole" that proper economizer operation leaves in the heating/cooling curve of Figure 3.5 can be a simple basis for diagnosing these problems.

A second service that the smart grid can provide is scheduling routine maintenance and extending equipment life. Another service is automated fault detection and diagnostics to remotely identify and diagnose real and potential problems before equipment fails and requires costly repairs. Improved maintenance would also reduce emissions of chlorofluorocarbon (CFC) refrigerants for units not yet using hydro-chlorofluorocarbons (HCFCs) that minimize such impacts.

The diagnostic services can be delivered by a smart grid in two ways. The first is by sending the necessary data to the utility or a third party for analysis at a central location. The other is by downloading the required software applications onto a platform within the customer premise. The former requires an enhanced communications network capability. The latter requires a suitable platform in the home or building energy-management system with the processing power and storage to accomplish the analysis.

The large, complex HVAC systems prevalent in larger commercial buildings are custom designed and built-up from chillers, boilers, cooling towers, and multi-zone air-handling units connected by water and air distribution systems. The nature of HVAC system design and operation in large buildings makes them less amendable to the kinds of simple, uniform diagnostics that can be supported by a smart grid. Additionally, large commercial buildings often have a dedicated staff or contractor and onsite equipment to set up and perform diagnostics and operations functions that can help detect problems, which could lead to energy savings (Brambley et al. 2009). Thus, while the potential for diagnostics to save energy in these buildings is as large, or even larger, than in homes and small commercial buildings, we do not ascribe the potential savings for large buildings to a smart grid.

Of course, diagnosing a problem does not result in energy savings unless the problem actually gets fixed. Anecdotal evidence suggests that pointing out problems and providing an estimate of the amount of energy being wasted may be insufficient to spur repairs in commercial buildings. Thus, it may be necessary to couple detection of problems with utility programs that affect the needed repairs. Linkage 
with energy efficiency or demand response programs that involve the relevant end uses is one such possible mechanism, particularly where utility efficiency investments are involved.

An important consideration pertains to data access and consumer privacy. Because of the sensitive nature of some of the data involved, use or transmission of the data outside the customer's premises should require the full knowledge and consent of the consumer. When utility or third-party investment is involved this may be a programmatic requirement. A solution is conducting diagnostics with software applications within the customer's premises, but this requires timely access to data from the meter and demand response controls. These are important smart grid policy questions that must be addressed to guide development of this mechanism.

The direct reductions in electricity and associated $\mathrm{CO}_{2}$ emissions calculated in Attachment 1 , Appendix C, and shown in Table 3.5 are based on the literature review and the author's experience. These are based on our estimate that potential reductions of $15 \%$ in the residential sector for heating and cooling electricity (with a range of $10 \%$ to $20 \%$ ) and $20 \%$ in small/medium commercial building sectors for HVAC and lighting (with a range of $10 \%$ to $30 \%$ ) are achievable through implementation of smart grid technologies. Large commercial buildings are excluded for the reasons described above. No indirect reductions in operational or capital costs are expected.

A discussion of the basis for these estimates from the literature examined and more details on the building diagnostics are provided in Attachment 1, Mechanism C.

Table 3.5. Estimated Direct Impacts for Enabling Mass Deployment of Diagnostics in Residential and Small/Medium Commercial Buildings

\begin{tabular}{|c|c|c|c|c|c|c|c|c|}
\hline \multirow[b]{3}{*}{$\begin{array}{c}\text { Est. } \\
\%\end{array}$} & \multicolumn{4}{|c|}{ Reduced Energy Consumption (2030) } & \multicolumn{4}{|c|}{ Electric Sector Annual Reductions (2030) } \\
\hline & \multirow[b]{2}{*}{$\begin{array}{c}\text { Low } \\
\%\end{array}$} & \multirow[b]{2}{*}{$\begin{array}{c}\text { High } \\
\%\end{array}$} & \multicolumn{2}{|c|}{ Baseline Electricity Consumption } & \multicolumn{2}{|c|}{ Energy } & \multicolumn{2}{|c|}{ Carbon Emissions } \\
\hline & & & End-Use Sector(s) & $\left(10^{9} \mathrm{kWh} /\right.$ year $)$ & $\begin{array}{c}\% \text { of } \\
\text { United } \\
\text { States }\end{array}$ & $\begin{array}{c}\left(10^{9} \mathrm{kWh} /\right. \\
\text { year })\end{array}$ & $\begin{array}{c}\% \text { of } \\
\text { United } \\
\text { States }\end{array}$ & $\begin{array}{c}(\mathrm{MMT} / \\
\text { yearr) }\end{array}$ \\
\hline 15 & 10 & 20 & $\begin{array}{l}\text { Residential (Heat Pump \& Air } \\
\text { Conditioner) }\end{array}$ & 331 & 3 & 152 & 3 & 90 \\
\hline 20 & 10 & 30 & $\begin{array}{l}\text { Small/Medium Commercial } \\
\text { Buildings (HVAC + Lighting) }\end{array}$ & 510 & & 102 & & 0 \\
\hline
\end{tabular}

\subsubsection{Measurement \& Verification for Energy Efficiency Programs}

The precision measurements that could be obtained by leveraging a smart grid could be used to provide additional value by offering $M \& V$ of energy savings from end-use efficiency programs on a realtime basis, for all participants, with great transparency and accuracy in the calculation of $r$ energy and $\mathrm{CO}_{2}$-emission reductions. Most evaluations of utility energy efficiency programs today are based on three general approaches:

- Stipulation of energy savings, based on simple in-situ or bench-scale tests that compare consumption with and without the efficiency measure, combined with assumed average operating times for appliances and equipment (that may be obtained from metered patterns) 
- Analysis of monthly electricity bills, comparing before and after periods, normalized for outside temperature effects, to estimate savings

- Short-term metering, as recommended by the International Performance Measurement \& Verification Protocol (IPMVP) in increase transparency and reduce risk (EVO 2007).

Stipulation of savings is particularly applicable for process loads such as motors, where use schedules are well understood, and similarly for lighting systems and electronics (if heating and cooling impacts can be ignored).

The PRISM-type methods discussed in Section 3.1.3 were developed specifically for the purpose of energy efficiency program evaluations, particularly those involving heating and cooling efficiency. For this application, they are typically employed to fit two- or three-part linear models to monthly billing data from a period prior to an efficiency retrofit, and again for a period after a retrofit. The consumption for both pre- and post-retrofit periods can be adjusted using the models to reflect a normal weather year. The difference between the normalized consumption for the two time periods is the savings attributed to the retrofit. These methods are desirable for programs that involve large numbers of buildings because, even for program targets other than heating and cooling, changes in other end uses, such as lighting, reduce internal heat gains and reduce cooling but increase heating. This interaction is taken into account by the methodology. Aside from the methodological difficulties, the principal limitation of this approach is estimating small levels of savings with confidence, amidst large overall consumption totals.

More detailed short-term monitoring is recognized by the IPMVP as a superior approach that overcomes this shortcoming by eliminating the need to make assumptions about use schedules and avoiding issues surrounding the difference between bench-scale tests and actual energy use in the field. If the short-term monitoring includes heating and cooling end uses, then the effects of changed internal heat gains on net savings can be properly accounted for as long as the data collected captures a full seasonal swing of weather. This approach does require significant labor to install metering equipment and collect the data, thus it is typically applied to a relatively small, random sample of participants over a short time period.

An approach that leverages smart grid communications and controls, as described in Section 3.1.3, can provide many of the benefits of short-term monitoring, but without the costs for field labor to install monitoring equipment and collect data. If it can be standardized and automated, it can be integrated into utility billing systems as a routine procedure. Once the procedures are developed and programmed, the cost to deploy it should be relatively independent of the number of customers involved, or the duration over which it is used. Employing it for all program participants eliminates the labor costs for developing samples and for recruiting participants for field measurement.

A review of the literature on efficiency program $M \& V$ is described in Attachment 1, Mechanism $C$, and briefly summarized here. Currently, $3 \%$ of total program costs are typically allocated for traditional M\&V (DOE/FEMP 2009). The cost of conducting M\&V by leveraging smart grid assets is unknown. Certainly, all labor involved will not be eliminated, but if high-quality $\mathrm{M} \& \mathrm{~V}$ approaches are desired, it could displace a significant fraction of the cost of approaches, such as short-term metering. If these costs are assumed to represent a third of the overall costs of $\mathrm{M} \& \mathrm{~V}$, then a savings of $1 \%$ of program operational costs could be realized. 
Using this estimate, if the savings were reinvested to obtain further cost-effective energy efficiency at an average cost of $8.8 \notin / \mathrm{kWh}$, potentially $0.5 \%$ of the electric sector energy and associated $\mathrm{CO}_{2}$ emissions could be achieved as indirect reductions, as indicated in Table 3.6. This relatively modest savings assumes that these $M \& V$ approaches apply only to the residential and small/medium commercial building sectors (less than 50,000 $\mathrm{ft}^{2}$ in floor area). It also assumes that energy efficiency programs are operated to achieve a 10\% overall improvement in energy efficiency for these customer segments. Larger buildings and industrial customers are assumed to warrant more sophisticated and specially designed $\mathrm{M} \& \mathrm{~V}$ approaches, and no attribution of savings from the programs is included in this estimate.

Table 3.6. Estimated Indirect Impacts of Measurement \& Verification for Energy Efficiency Programs Leveraging a Smart Grid

\begin{tabular}{|c|c|c|c|c|c|c|c|c|c|}
\hline \multirow[b]{3}{*}{$\begin{array}{c}\text { Est. } \\
\%\end{array}$} & \multicolumn{4}{|c|}{ Avoided Expenditure Reinvested to Save Carbon (2030) } & \multirow[b]{3}{*}{$\begin{array}{c}\text { Savings } \\
\left(10^{9} \$\right)\end{array}$} & \multicolumn{4}{|c|}{ Electric Sector Annual Reductions (2030) } \\
\hline & \multirow[b]{2}{*}{$\begin{array}{c}\text { Low } \\
\%\end{array}$} & \multirow[b]{2}{*}{$\begin{array}{l}\text { High } \\
\%\end{array}$} & \multicolumn{2}{|l|}{ Baseline Captial Expenditure } & & \multicolumn{2}{|c|}{ Energy } & \multicolumn{2}{|c|}{ Carbon Emissions } \\
\hline & & & Investment & $\left(10^{9} \$\right)$ & & $\begin{array}{l}\% \text { of } \\
\text { United } \\
\text { States }\end{array}$ & $\begin{array}{c}\left(10^{9} \mathrm{kWh} /\right. \\
\text { year })\end{array}$ & $\begin{array}{l}\% \text { of } \\
\text { United } \\
\text { States }\end{array}$ & $\begin{array}{c}\text { (MMT/ } \\
\text { year) }\end{array}$ \\
\hline 1 & 0 & 2 & $\begin{array}{l}\text { 10\% Energy Efficiency, Residential } \\
\text { @ } 8.8 \notin / \mathrm{kWh}, 10 \text {-Year Life }\end{array}$ & 152 & 1.5 & & & & \\
\hline 1 & 0 & 2 & $\begin{array}{l}\text { 10\% Energy Efficiency, } \\
\text { Small/Medium Commercial } \\
\text { Buildings @ 8.8غ/kWh,10-Year Life }\end{array}$ & 75 & 0.8 & 0.5 & 26 & 0.5 & 15 \\
\hline
\end{tabular}

There is obviously considerable uncertainty about whether these cost savings are achievable. We acknowledge this by indicting a range of potential cost savings that includes zero. Pending further research to better quantify the savings potential, and the cost of developing automated methodologies, the reader is left to accept or reject the assertions made regarding this mechanism.

We also estimate the direct impact for the additional energy efficiency that can be deployed as a result of the improved quality of $\mathrm{M} \& \mathrm{~V}$. This stems from the methodological advantages that approaches based on smart grid have over stipulation methods and methods based on analysis of monthly bills, which are otherwise assumed to remain viable and common approaches to $\mathrm{M} \& \mathrm{~V}$. Among other advantages, this allows the separation of the effects of physical thermal performance of a building and equipment from the behavior-driven effects of appliance and equipment loads and thermostat settings. This separation provides deep insight into how and why savings occur for any given technology, and provides an engineering basis for estimating savings in new construction, whereas prior baseline performance does not.

Quality also stems from the ability to ensure persistence_of savings for new energy efficiency technologies with considerable potential, like heat pump water heaters, whose long-term performance is uncertain, which limits its penetration. These are usually actively controlled technologies that can fail in modes that reduce savings but shield the user from impacts of lost amenity, as opposed to passive technologies integral to building envelopes, for example. This benefit of ensuring persistence is beyond that which short-term metering can provide.

These effects are extraordinarily difficult to estimate. The additional reductions in electricity and $\mathrm{CO}_{2}$ emissions resulting from the deployment of additional, marginally cost-effective energy efficiency technologies calculated in Attachment 2 are based on EPRI's estimate of 7\% (EPRI 2009), other 
information in the literature review, and the author's experience. Potential direct reductions of $7 \%$ are estimated for cooling and electric heating in the residential sector (with a range of 5\% to 20\%), and 7\% in the small/medium commercial building sectors for HVAC and lighting (with a range of $5 \%$ to $20 \%$ ).

Using this estimate, potential reductions of $1 \%$ of the electric sector energy and associated $\mathrm{CO}_{2}$ emissions could be achieved, as indicated in Table 3.7. These relatively modest savings assume that these $M \& V$ approaches apply only to the relatively sophisticated types of HVAC equipment and systems in the residential and small/medium commercial building sectors (less than 50,000 $\mathrm{ft}^{2}$ in floor area) and lighting in those commercial buildings. Larger buildings and industrial customers are assumed to warrant more sophisticated and specially designed $\mathrm{M} \& \mathrm{~V}$ approaches, and no attribution of savings from the programs is included in this estimate.

Table 3.7. Estimated Direct Impacts of Measurement \& Verification for Energy Efficiency Programs Leveraging a Smart Grid

\begin{tabular}{|c|c|c|c|c|c|c|c|c|}
\hline \multirow[b]{3}{*}{$\begin{array}{c}\text { Est. } \\
\%\end{array}$} & \multicolumn{4}{|c|}{ Reduced Energy Consumption (2030) } & \multicolumn{4}{|c|}{ Electric Sector Annual Reductions (2030) } \\
\hline & \multirow[b]{2}{*}{$\begin{array}{c}\text { Low } \\
\%\end{array}$} & \multirow[b]{2}{*}{$\underset{\%}{\mathrm{High}}$} & \multicolumn{2}{|c|}{ Baseline Electricity Consumption } & \multicolumn{2}{|c|}{ Energy } & \multicolumn{2}{|c|}{ Carbon Emissions } \\
\hline & & & End-Use Sector(s) & $\left(10^{9} \mathrm{kWh} /\right.$ year $)$ & $\begin{array}{c}\% \text { of } \\
\text { United } \\
\text { States }\end{array}$ & $\begin{array}{l}\left(10^{9} \mathrm{kWh} /\right. \\
\text { year })\end{array}$ & $\begin{array}{c}\% \text { of } \\
\text { United } \\
\text { States }\end{array}$ & $\begin{array}{l}\text { (MMT/ } \\
\text { yearr) }\end{array}$ \\
\hline 7 & 5 & 20 & $\begin{array}{l}\text { Residential (Heat Pump \& Air } \\
\text { Conditioner) }\end{array}$ & 331 & \multirow[b]{2}{*}{1} & 59 & \multirow{2}{*}{1} & \multirow{2}{*}{35} \\
\hline 7 & 5 & 20 & $\begin{array}{l}\text { Small/Medium Commercial } \\
\text { Buildings (HVAC + Lighting) }\end{array}$ & 510 & & 155 & & \\
\hline
\end{tabular}

\subsubsection{Shifting Load to More Efficient Generation}

A smart grid facilitates shifting load from peak load to shoulder or off-peak-load periods using demand response and distributed generation and storage. Doing so with demand response or storage and can save energy and carbon emissions, depending upon the mix of base, intermediate, and peak load generating resources being used at any given time to serve customers for a given utility. This is illustrated in

Figure 3.6, which shows an actual coal-based Midwestern U.S. utility's load duration curve ${ }^{1}$ on the left axis and a colored block whose height on the right axis indicates the carbon footprint of the marginal power plant operating during any given hour of the year.

The carbon footprint for the natural-gas-fired peak load power plants is almost as high as that for the coal-fired base-load power plants. This is because the heat rate (energy conversion efficiency from fuel) for simple cycle combustion turbines (CTs) used to supply peak loads is generally low because their limited annual run-time is too low to justify the more expensive, efficient combined-cycle power plant that serves intermediate loads. Thus, every kilowatt-hour saved is not "born equal" in terms of its carbon impact, and demand response that shifts load from peak load times to intermediate load periods (or, less frequently, to base load) will have an important carbon impact that is worth taking into account. This simple example avoids the much greater complexity of accounting for carbon emissions as renewable generation becomes a significant contributor in the future.

${ }^{1}$ A load duration curve is used to provide the relationship between generating capacity utilization by hour of a year (8760 hours) in decreasing increments by hour. 


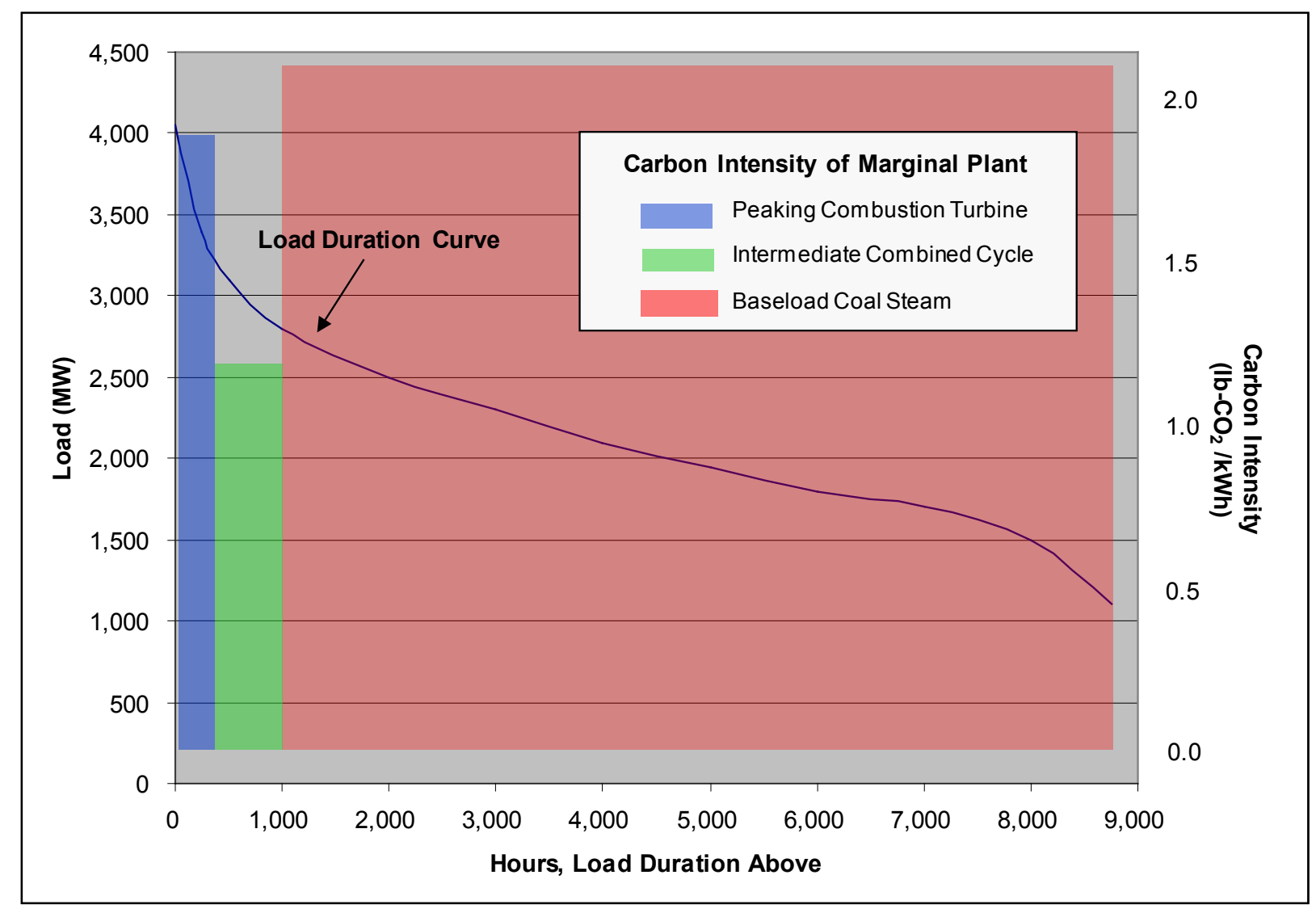

Figure 3.6. Load Duration Curve and Carbon Dispatch of a Typical Coal-Based Utility

The smart grid can provide reductions in primary energy and $\mathrm{CO}_{2}$ emissions by shifting peak load to more efficient lower emission base and intermediate generation resources. Load shifting enabled by a smart grid can shift electricity production from less efficient peak load generating resources (less than $30 \%$ ) to more efficient intermediate resources $(\sim 40 \%)$ that have lower carbon emissions per unit of energy supplied. In cases where the load is shifted to base-load power plants that are not coal-fired, even greater carbon savings can be realized. Utility programs have shown that shifting load from peak load generating power plants to more efficient off-peak-load power plants provides such reductions: the California "Shift \& Save" quantifies the reduced $\mathrm{CO}_{2}$ emissions at between $10 \%$ and $20 \%$. The carbon footprint of base-load generation is likely to be reduced in the future as more renewable and clean-coalfired power plants enter the system to join nuclear and natural-gas-fired power plants to displace current coal-fired power plants.

The estimation of energy and carbon benefits achievable by load shifting is challenging because of the highly dynamic nature of the power plant dispatch options that provide literally thousands of options for re-arranging the generation mix, and the corresponding generating efficiency and carbon intensity of the input fuel. To estimate the potential reductions, a simplified analysis was conducted using load duration curves for each of 12 North American Electric Reliability Corporation (NERC) sub-regions. Sufficient load was shifted from high demand hours to provide a $10 \%$ reduction in peak load in each subregion, or in the amount of capacity of natural-gas-fired CTs in each sub-region, whichever is less. On average, this involved shifting load for 168 hours per year. 
In general, the load was shifted to natural-gas-fired combined-cycle power plants, which reduces energy input and $\mathrm{CO}_{2}$ emissions because of their lower heat rates (higher fuel efficiency). For three of the regions in which there is a lower proportion of combined-cycle capacity and coal-fired power plants (East Central Area Reliability Coordinating Agreement, Mid-America Interconnected Network, and MidContinent Area Power Pool [MAPP]), a portion of the energy was shifted to coal-fired power plants $(50 \%, 40 \%$, and $80 \%$, respectively). This produces energy savings because of their lower heat rates, but causes higher $\mathrm{CO}_{2}$ emissions because of the higher carbon content of coal compared to natural gas.

The results of the analysis described in Attachment 1, Mechanism E, are summarized here. The reductions in electricity and $\mathrm{CO}_{2}$ emissions calculated in Attachment 2 for this analysis are shown in Table 3.8. The estimated potential from the load-shifting capabilities of smart grid technologies are small: a direct reduction of $0.04 \%$ in total electricity supplied to the grid (with a range of $0.02 \%$ to $0.06 \%$ ), and $0.03 \%$ reduction in associated $\mathrm{CO}_{2}$ emissions (approximately $75 \%$ of the electricity reduction). No indirect reductions are expected.

Table 3.8. Estimated Direct Utility Energy and Carbon Reductions for Shifting Load to More Efficient Generation

\begin{tabular}{|c|c|c|c|c|c|c|c|c|}
\hline \multicolumn{5}{|c|}{ Reduced Energy Consumption (2030) } & \multicolumn{4}{|c|}{ Electric Sector Annual Reductions (2030) } \\
\hline \multirow[b]{2}{*}{$\begin{array}{c}\text { Est. } \\
\%\end{array}$} & \multirow[b]{2}{*}{$\begin{array}{c}\text { Low } \\
\%\end{array}$} & \multirow[b]{2}{*}{$\begin{array}{c}\text { High } \\
\%\end{array}$} & \multicolumn{2}{|c|}{ Baseline Electricity Consumption } & \multicolumn{2}{|c|}{ Energy } & \multicolumn{2}{|c|}{ Carbon Emissions } \\
\hline & & & End-Use Sector(s) & $\left(10^{9} \mathrm{kWh} /\right.$ year $)$ & $\begin{array}{c}\% \text { of } \\
\text { United } \\
\text { States }\end{array}$ & $\begin{array}{c}\left(10^{9} \mathrm{kWh} /\right. \\
\text { year })\end{array}$ & $\begin{array}{c}\% \text { of } \\
\text { United } \\
\text { States }\end{array}$ & $\begin{array}{c}\text { (MMT/ } \\
\text { yearr) }\end{array}$ \\
\hline 0.04 & 0.02 & 0.06 & Total Electric Supply & 4968 & 0.04 & 2 & 0.03 & 1 \\
\hline
\end{tabular}

These reductions are quite small. This is fundamentally because of the relatively few hours per year the load needs to be shifted to produce a 10\% reduction in peak load (168 hours on average), and the average power shifted during those hours is only about 5\% (approximating the area of the "wedge" shifted as a triangle). So, the overall energy shifted is correspondingly small (about $0.1 \%$ of the total generation), and in the limit this could only produce $0.1 \%$ savings even if the generation used to meet it were entirely renewable.

A dispatch algorithm that shifts load more frequently could produce larger reductions. It would presumably have energy and $\mathrm{CO}_{2}$ reductions as its primary objective, rather than peak load management. Since demand response is limited by the willingness of participants to forgo some amenity or service value, it may not be possible to utilize demand response assets on a daily basis in such an algorithm. Energy storage could play a much more frequent role in such a dispatch algorithm, as long as such frequent use would not reduce its lifetime. Among the options for storage, compressed air or pumped hydro storage could play a much more significant role than battery-based storage for this purpose.

\subsubsection{Support Additional Electric Vehicles and Plug-In Hybrid Electric Vehicles}

This topic examines how advanced load management technologies for EV, "smart charging," can improve the overall national energy efficiency and reduce carbon emissions of LDV transportation. This mechanism is summarized here, with detail on the literature review and conclusions provided in Attachment 1. 
Replacing gasoline-fueled LDVs with vehicles that derive a significant fraction of their energy from electricity is one option for reducing our dependence on foreign oil and the carbon footprint of transportation at the same time. Compared to burning gasoline in an engine at relatively low operational efficiency, generating electricity with a power plant and putting that energy into an on-board battery to propel a vehicle it is significantly more fuel-efficient. It is estimated that with today's mix of power plants and vehicles, this would provide an approximate 30\% improvement in energy consumption per VMT and a $27 \%$ reduction in $\mathrm{CO}_{2}$ emissions, while reducing imports of foreign oil by $52 \%$ (KintnerMeyer et al. 2007).

PHEVS are often cited as a solution that bridges the need for better transportation efficiency and consumers desire for travel range. With the advent of better battery technology, PHEVs may be supplanted by EVs, but the reduced fuel and emissions are the same per VMT when powered with electricity.

Analysis has shown that with today's load shape and generation capacity, it should be possible to supply over $70 \%$ of the energy ${ }^{1}$ for the U.S. LDV fleet (cars, vans, SUVs, and light trucks) without building additional generation or transmission - if their charging times are carefully managed to strictly avoid charging during peak load hours. If this can be accomplished, there is downward pressure on electricity prices because the cost of the existing grid infrastructure is spread over more unit sales of energy (Scott et al. 2007). That will help keep electricity an affordable and viable alternative to gasoline.

The ability to manage the charging time period and shift the vehicle load off peak is the enabling characteristic of smart charging technologies that would be supplied by a smart grid. The analysis documented in Attachment 1, Mechanism F, focuses on determining the impact of a smart grid on achieving savings from PHEVs or EVs. First, it must be recognized that the primary investment from which any savings are derived is from the vehicle itself, not a smart grid. While a smart grid is designed to help keep electricity prices down, and that will help electric-powered vehicles penetrate, the dominant influence on their penetration is likely to be cost and performance in the eyes of the consumer. That is primarily driven by battery technology.

The impacts of a smart grid analyzed here focus on the question of how many additional PHEVs/EVs can be supported by using smart charging. Driving data from a large sample of vehicles was used to estimate when vehicles arrive at home in the evening, and it was assumed that charging of their batteries would immediately commence at 120 volts. On the average day in the peak load season, many of these vehicles begin charging while the grid is still in a peak load condition. The (base case) analysis first determined how many vehicles could be accommodated before they caused the total load to exceed the available excess generating capacity on that day was exceeded (less reserve margins). ${ }^{2}$ This was then compared to the number of vehicles that could be supported in each of 13 regions comprising the entire United States with smart charging, and the difference attributed to a smart grid.

The result is that smart charging raises the share of electric VMT by 9 percentage points-from $64 \%$ to $73 \%$ of the LDV fleet. This allows the grid to support 18 million more PHEVs and EVs beyond the 140 million supportable with unmanaged charging.

\footnotetext{
${ }^{1}$ Or, equivalently, $70 \%$ of the VMT.

${ }^{2}$ This analysis implicitly assumes that since both are driven by population growth, to a first order the number of vehicles grows at the same rate as the electric generating capacity.
} 
The reductions in electricity and $\mathrm{CO}_{2}$ emissions calculated in Attachment 2 are based on this estimate. The estimated potential direct impacts are a net (petroleum minus electricity) reduction of $3 \%$ (with a range from $2 \%$ to $5 \%$ ) in the energy consumption for LDV transportation at some point in the future, if EV/PHEV penetrations reach the $73 \%$ level discussed above. To place this in perspective with the potential impacts of the other mechanisms in this report, we translate this into equivalent reductions in U.S. electric sector energy and associated emissions of 3\%, as shown in Table 3.9. These percentage impacts are nearly the same because the energy for LDVs and electric power consumption are nearly the same. No indirect reductions in electricity or capacity are expected.

Table 3.9. Estimated Direct Utility Energy and Carbon Reductions from Supporting Additional Electric Vehicles and Plug-In Hybrid Electric Vehicles

\begin{tabular}{|c|c|c|c|c|c|c|c|c|}
\hline \multirow[b]{3}{*}{$\begin{array}{c}\text { Est. } \\
\%\end{array}$} & \multirow[b]{3}{*}{$\begin{array}{c}\text { Low } \\
\%\end{array}$} & \multicolumn{3}{|c|}{ Reduced Energy Consumption (2030) } & \multicolumn{4}{|c|}{ Electric Sector Annual Reductions (2030) } \\
\hline & & \multirow[b]{2}{*}{$\begin{array}{c}\text { High } \\
\%\end{array}$} & \multicolumn{2}{|c|}{ Baseline Electricity Consumption } & \multicolumn{2}{|c|}{ Energy } & \multicolumn{2}{|c|}{ Carbon Emissions } \\
\hline & & & End-Use Sector(s) & $\left(10^{9} \mathrm{kWh} /\right.$ year $)$ & $\begin{array}{l}\% \text { of } \\
\text { United } \\
\text { States }\end{array}$ & $\begin{array}{c}\left(10^{9} \mathrm{kWh} /\right. \\
\text { year })\end{array}$ & $\begin{array}{l}\% \text { of } \\
\text { United } \\
\text { States }\end{array}$ & $\begin{array}{c}\text { (MMT/ } \\
\text { yearr) }\end{array}$ \\
\hline 3 & 2 & 5 & $\begin{array}{l}\text { Electricity Equivalent of Light } \\
\text { Vehicle Transportation (cars, vans, } \\
\text { SUVs, light trucks) }\end{array}$ & 5135 & 3 & 139 & 3 & 82 \\
\hline
\end{tabular}

Of course, electric-powered vehicles are not the only potential solution to the energy and carbon footprint of transportation. If an alternative solution predominates, the reductions may not be fully attributable to a smart grid. Bio-fuels are another important option, particularly coupled with cellulosicconversion technology and clean diesel engines. Hydrogen is also an option, but if it is produced with renewable electricity, or coal-fired power plants with carbon-sequestration, conversion losses of 50\% hinder its potential. Nuclear power plants could be used to produce hydrogen if costs were low enough to compensate for the conversion losses. They could also be used to produce electricity, but then managing charging with a smart grid would become even more important because nuclear power plants are not suited to ramp up and down to follow load.

There is currently much discussion about whether 120 -volt charging will be the norm. In large vehicles like SUVs, charging at that voltage for a 30-mile range can take 12 hours or more. Shorter charging times may be desired by consumers, in which case 240 -volt charging may become the standard. 240 -volt outlets in garages of new homes are already required in California for this reason. To a first order, charging at 240 volts doubles the peak load impact of unmanaged charging, and therefore cuts the number of vehicles that can be supported with unmanaged charging in half. This reduces the fraction of electric VMTs that can be supported by the grid before smart charging is required to $32 \%$, raising the smart grid's impact from $9 \%$ to $41 \%$, more than quadrupling the estimated reductions to $13 \%$. This highlights an issue that unmanaged PHEV charging may set new system peaks in some regions, rather than be "perfect valley-filling" solution under a managed charging paradigm.

This analysis ignores the possible additional benefits of managing the charging of EVs and PHEVs, and potentially discharging them as well, to provide ancillary services. This could provide an indirect benefit by reducing the costs for ancillary services required to integrate high levels of renewable wind and solar generation. These benefits could be substantial if there are enough vehicles to drive down the 
market cost of regulation below that of power plants. The impacts on the lifetime of using vehicle batteries this way is not yet known. Analyzing these potential impacts is left to future analysis.

\subsubsection{Conservation Voltage Reduction and Advanced Voltage Control}

This subsection describes how the smart grid can increase the efficiency of electricity delivery by managing the electric service voltage seen by end-use customers to reduce the distribution system losses and reduce the energy consumption of customer loads. End-use energy consumption has been shown to drop when the electric service voltage is reduced. This strategy, termed conservation voltage reduction (CVR), occurs primarily because the energy consumption of certain end-use loads such as incandescent lights and certain electronics go down as the voltage is decreased.

Conversely, electric losses in distribution systems tend to increase as voltage drops, because motors and other constant power loads tend to draw more current to compensate, and losses are proportional to the square of the current. Electric distribution system losses average around 5\% and increase to $8 \%$ or more during peak load periods when voltage drops and current increases. A smart grid's measurement and communication capabilities provide an opportunity to continually optimize tradeoffs in service voltage and energy use by precisely controlling voltage within acceptable limits. This optimization process, which includes CVR, is advanced voltage control.

The drop in voltage along the length of a feeder is illustrated in Figure 3.7 under peak and minimum load conditions. The voltage drops because of the power losses in the conductors and equipment on the feeder. The higher the load, the more current flows through the feeder and the higher the resultant voltage drop. The voltage standard for the United States for a single phase at a residential customer meter ranges from 126 volts to 114 volts, per ANSI C84.1 (ANSI 1996). Voltages higher or lower than that have the potential to damage customer equipment.

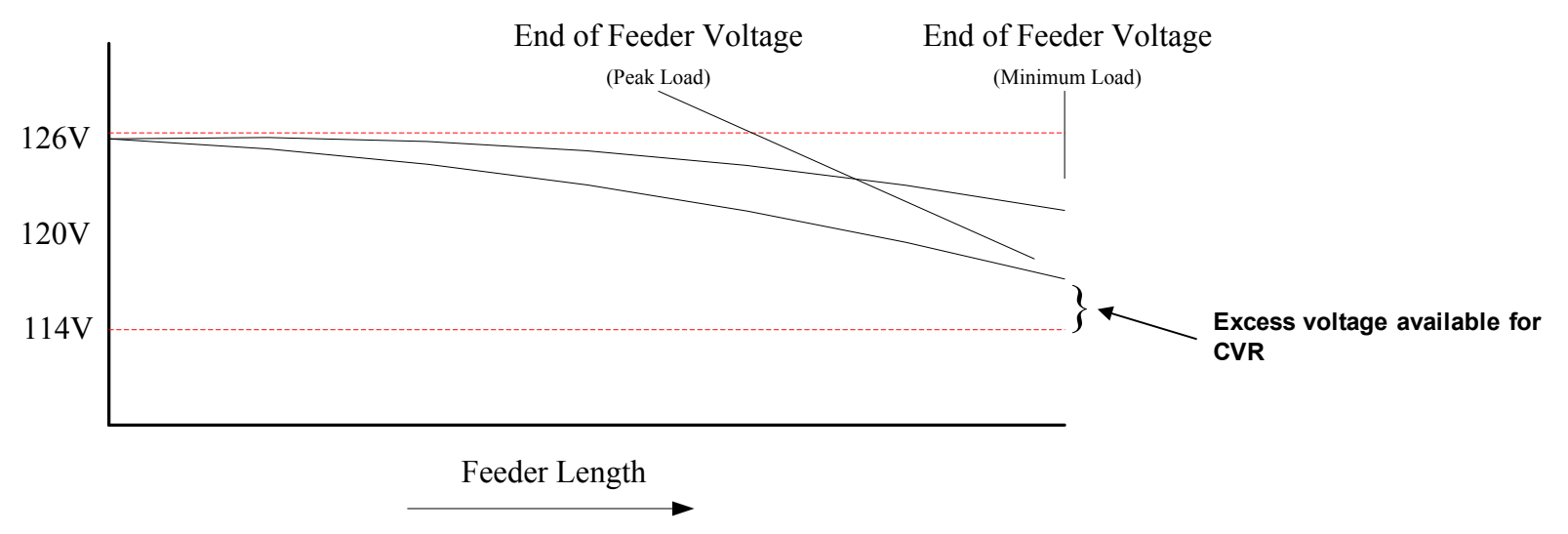

Figure 3.7. Voltage Drop Along a Feeder at Peak and Minimum Loads

Distribution operators maintain voltages for all customers within these limits by adjusting voltage at the substation transformers and voltage regulators at head of the feeder. In particularly heavily loaded feeders, additional voltage regulators are sometimes added along the length of the feeder to adjust the voltage. Typically, the voltage at the head of the feeder is set somewhat lower than the maximum 126volt level, but a safety factor in the form of some excess voltage is provided at the end of the feeder. 
CVR simply requires adding a measurement of voltage at the end of the feeder, or estimating it using load flow calculations. This low requirement for additional capital investment makes CVR an inexpensive efficiency measure. Then the voltage regulator at the head of the feeder is continually controlled to maintain the end-of-line voltage at the minimum level. This reduces the instantaneous power drawn by any load with constant impedance characteristics, and reduces overall customer energy consumption. Loads controlled by thermostats, like heating, cooling, and refrigeration, may not reduce consumption in proportion to the reduction in power because they operate with slightly longer cycles to satisfy the thermostat.

Winding losses in motors and transformers are also reduced, and motors may operate at a higher efficiency if their operation shifts to a more efficient operating point. On the other hand, motors in many applications also tend to maintain constant power output. This causes them to draw more current to compensate for the drop in voltage, which does nothing to reduce the electrical power drawn. This actually increases losses in the distribution system. So, while the technology behind CVR is relatively simple, understanding the impacts of CVR is somewhat complicated.

On heavily loaded or long rural feeders it is not always possible to maintain the proper voltage by adjustments of the substation voltage alone. In many cases, this is caused by heavy motor or airconditioning loads with poor power factors. The lag of the current behind the voltage (indicated by the power factor) requires additional current to deliver a given amount of power, with attendant decrease in voltage. Shunt capacitors can be added to compensate for the poor power factor.

Figure 3.8 shows an example of a radial distribution feeder that has a very large voltage drop. To accommodate the large voltage drop, a shunt capacitor is placed approximately $60 \%$ down the length of the line. This has the effect of reducing line losses and voltage drop along the feeder. Today, these capacitors are usually continually active or they may be manually switched on and off. The smart grid will involve an extension of distribution SCADA systems along the length of the feeder so that these capacitors can be automatically controlled to compensate for the variation in the voltage throughout the day based on local voltage measurements. As shown in Figure 3.8, capacitor control can make extra voltage available for CVR by providing truly advanced voltage control to enhance load and loss reductions.

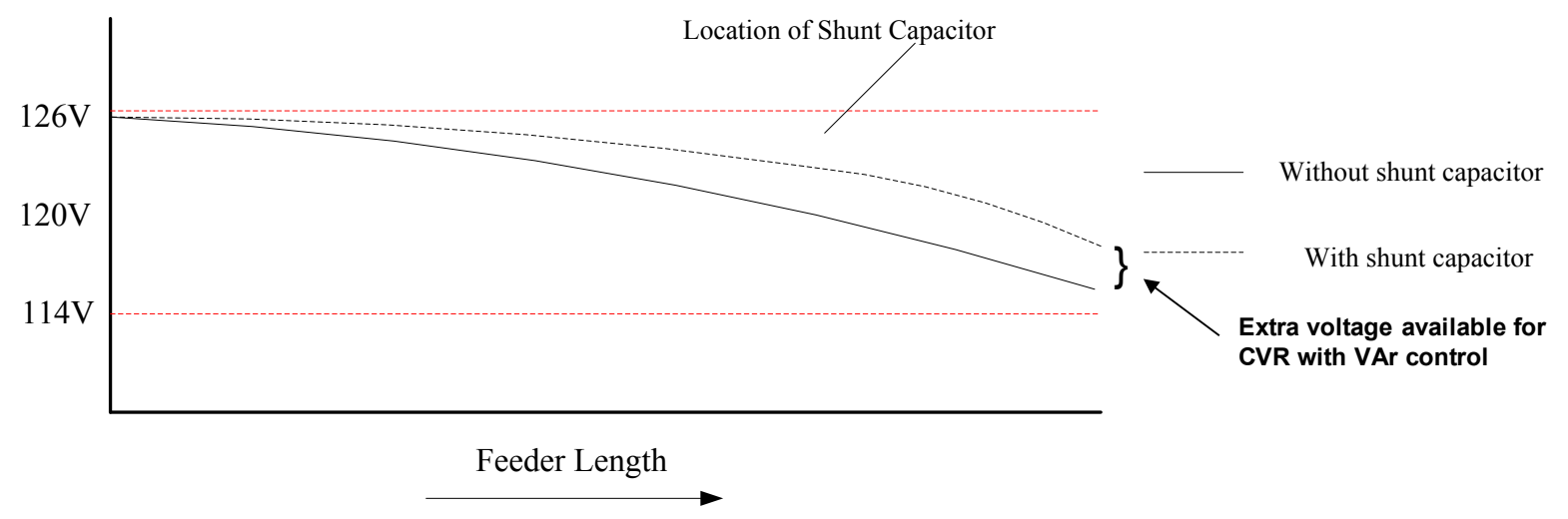

Figure 3.8. Effect of Reactive Power Control on Voltage Drop 
The literature review detailed in Attachment 1, Mechanism G, suggests that CVR alone has empirically proven itself to be a viable method to reduce the peak load on a distribution feeder as well as being an effective form of conservation. The most comprehensive field study involved 31 feeders at 10 different substations and 11 utilities in the Pacific Northwest; it showed that a 1\% change in distribution line voltage provided a $0.25 \%$ to $1.3 \%$ change in energy consumption, and that voltages could be reduced from $1 \%$ to $3.5 \%$ (Beck 2007).

Accurate determination of the CVR effects on any given feeder must include analysis of the electrical load as well as the design of the distribution system. The design of the distribution feeders includes everything from line and cable types, line and cable configurations, use of voltage correction capacitors, and use of tap-changing voltage regulators for transformers. Thus, extrapolating the CVR results to estimate the national potential is difficult.

Using advanced voltage control, we estimate that it is possible to reduce the existing consumption of electricity by approximately $1 \%$ with little investment. Such functionality is generally considered basic to a smart grid, so here we are simply trying to quantify its potential. Deploying full advanced voltagecontrol technologies could potentially increase this from $3 \%$ to $4 \%$, which translates directly into substantial savings. The reductions in electricity and associated $\mathrm{CO}_{2}$ emissions calculated in Attachment 2 and shown in Table 3.10 are based on information from the literature review and the author's experience. It is estimated that a direct reduction of $2 \%$ in total electricity supplied to the grid, with a range of $1 \%$ to $4 \%$, can be achieved through implementation of smart grid technologies. No indirect reductions in electricity or capacity are expected.

Table 3.10. Estimated Direct Utility Energy and Carbon Reductions for Conservation Voltage Reduction and Advanced Voltage Control

\begin{tabular}{ccccccccc}
\hline & \multicolumn{3}{c}{ Reduced Energy Consumption (2030) } & \multicolumn{3}{c}{ Electric Sector Annual Reductions (2030) } \\
\cline { 3 - 9 } & & & \multicolumn{3}{c}{ Baseline Electricity Consumption } & \multicolumn{2}{c}{ Energy } & \multicolumn{2}{c}{ Carbon Emissions } \\
\hline & & & & $\%$ of & & $\%$ of \\
Est. & Low & High & & & United & $\left(10^{9} \mathrm{kWh} /\right.$ & United & (MMT/ \\
$\%$ & $\%$ & $\%$ & End-Use Sector(s) & $\left(10^{9} \mathrm{kWh} /\right.$ year) & States & year) & States & yearr) \\
\hline 2 & 1 & 4 & Total Electric Supply & 4968 & 2 & 99 & 2 & 59 \\
\hline
\end{tabular}

\subsection{The Smart Grid and Renewables}

A smart grid can help integrate renewable resources into the grid by designing price or incentive signals to engage demand response and distributed storage, including that from PHEVs, to manage and absorb the short-term fluctuations ("noise") in the total load in a service territory, instead of using power plants to manage/absorb these fluctuations. Currently, power plants are continually turned up and down to provide this load following service (termed regulation), which wastes fuel and increases wear and tear on the power plants. The increased penetration of renewable generation resources increases the need for regulation services, as projected for California (CAISO 2007).

Regulation is one form of ancillary services needed to stabilize the grid during normal operations, and the need for regulation is expected to increase in order to manage high penetrations of renewables. An illustrative example of this occurred in February 2008, when the Electric Reliability Council of Texas (ERCOT) had to curtail power to many interruptible customers because wind production suddenly fell 
1700 megawatts. The drop in output had been forecast, but occurred several hours earlier than expected, so power plants had not been scheduled for dispatch to provide the replacement energy and the ramping services to manage the transition. Some generation capacity is always held back but kept "hot" to handle a sudden contingency (spinning reserves), but this event exceeded the capacity of the spinning reserves and fast-acting non-spinning reserves to pick up the deficit in output. In addition, February is in the offpeak-load season in Texas when many power plants were down for scheduled maintenance. As a result of this deficit, grid frequency dropped quickly, and emergency curtailment contracts, mostly with large industrial customers, were called upon to drop load to prevent a potential blackout until additional power plants could be brought online.

These kinds of events are driven largely by errors in forecasts of renewable energy. They are a combination of probabilistic events, well described by the analogy to a "perfect storm," that power grid operators must plan to handle with little warning. In today's power grid with little renewable generation, contingency events are not infrequent, and the rules for good practice regarding how much capacity to have for regulation, spinning and non-spinning reserves, and emergency replacement energy (re-dispatch) have been well defined after decades of experience. Because these events are probabilistic, the quantity of services needed, which may be negligible initially, tends to accelerate as renewable resource penetration increases.

How much additional capacity is needed for these services, and how this quantity changes with the percentage of generation supplied by renewables, is the subject of considerable research. Most research conducted to date is focused on wind power, because it is penetrating much faster than solar power systems at present. A smart grid's demand response and distributed generation and storage assets can provide these services, easing operational stresses, and manage the increasing penetration of intermittent renewable resources.

To the extent that these assets (demand response, distributed generation, and distributed storage) can replace power plants in providing these services, extra plant capacity will not need to be constructed, and less fuel will be consumed. In Section 3.2.2, we estimate the direct impacts from the potential savings in fuel for the additional regulation services required by a 20\% RPS requirement met by wind power, by providing the extra regulation with a smart grid's demand response and/or distributed storage resources. We also estimate the indirect impacts of saving capital investment in power plant capacity for providing the extra spinning reserves needed.

We do not provide a separate estimate for meeting a 20\% RPS requirement with solar power generation for two reasons: wind power is expected to provide most of the needed additional RPS requirement, and research on the ancillary services required to meet an RPS requirement entirely or partially is relatively immature. To a first order, we assume the requirements are similar, and therefore the estimates in Section 3.2.2 for wind power also apply to a system with a mixture of wind and solar. ${ }^{1}$

Other smart-grid-enabled mechanisms for assisting the penetration of renewable generation, such as wide-area control and dynamic thermal rating schemes for transmission systems, are not analyzed in this report. Both of these could potentially increase the throughput capacity of existing transmission lines, and thereby reduce needs to construct transmission capacity in order to move renewable power long

\footnotetext{
${ }^{1}$ This neglects the generally beneficial effects of resource diversity, which is what a combination of wind and solar sources would provide.
} 
distances to urban load centers. Wide-area control involves using high-precision data from phasor measurement units and high-performance computing techniques to analyze the transmission grid and reconfigure it as needed in real time. In principle, this could allow some relaxation of restrictions on key transmission corridors due to stability limitations, because the grid could be reconfigured instantly to relieve a stability contingency. Wide-area control technology is a long-term technology development focus for smart grids at the transmission level. When it may become practical, and how much additional new transmission capacity to serve renewable generation could be avoided, is not yet clear.

Dynamic thermal rating schemes are available today. They use sensors to account for the actual local weather conditions when computing the thermal capacity limits on transmission line segments, instead of assuming worst-case conditions, as is the current practice. When and where the wind is blowing can lower conductor temperatures and thereby reduce line sag enough so that additional power can be delivered. How much avoided transmission capacity this promising technology can deliver in practice is uncertain. While it can increase throughput on specific lines under certain conditions, many transmission systems are constrained by stability limits rather than thermal limits. Even when wind power output is high, it may not be blowing sufficiently at a key constrained transmission segment to sufficiently increase the throughput sufficiently to accommodate the increased generation. Further research is required on this subject before such estimates can be made.

Another way a smart grid can assist renewable generation is to remove barriers that may limit its penetration. Aside from the cost hurdles associated with providing extra ancillary services, more absolute barriers are not generally unforeseen by experts, at least until the renewable portfolio reaches levels above $20 \%$. One example of such a barrier is explicitly addressed in Section 3.2.1 of this report: the limits to the amount of solar generation in neighborhoods, presumably from solar photovoltaic (PV) installations, before reverse power flow toward the substation occurs and distribution voltage control is lost (we do not attempt to ascribe savings associated with overcoming this barrier).

Perhaps the ultimate barrier that can be foreseen is the limit to the share of energy needs provided by renewable generation. Beyond ancillary services, other power plants will need to provide replacement energy for days and occasional weeks when renewable resources do not produce their average output. The first barrier is simply one of cost for the replacement reserve capacity. Although demand response is unlikely to produce significant energy for days at a time, a smart grid's storage resources may be able to provide a day or more, and backup distributed generation could provide supply over an even longer period.

An absolute limit on the share of energy that can be produced from renewable generation is eventually reached when the fuel consumed by power plants to supply replacement energy becomes the only non-renewable production by the grid. At that point, the addition of further renewable capacity does not result in a corresponding increase in production of renewable energy. ${ }^{1}$ A smart grid aggressively managing storage resources, potentially including batteries in PHEVs and EVs, becomes essential in overcoming this limit. Estimating when this limit is reached and the corresponding share of renewable electricity production becomes significant is extremely complex and beyond the scope of this report.

\footnotetext{
${ }^{1}$ Unless it is from a new form of renewable generation that increases diversity, or that is not variable. Geothermal, tidal, and wave energy are some examples.
} 
The following subsections summarize the two mechanisms that relate to the renewable energy application (the full text discussing the two mechanisms is contained in Attachment 1).

\subsubsection{Support Penetration of Renewable Solar Generation}

This section provides a simple estimate of how much solar generation can exist in a typical residential neighborhood, downstream from the substation, before reverse power flow can be expected.

The integration of solar PV generation at high penetration in distribution systems will eventually require two-way flows of electric power toward the substation when the energy from solar PVs exceeds the downstream customer loads. The voltage control and short-circuit protection schemes used by distribution systems today were not designed to operate with reverse/upstream power flow. A smart grid's assets can reduce these limitations and improve system stability and safety through dynamic protection schemes, voltage regulation and control, energy storage, and the provision of dynamic reactive power. DOE has a very active program that focuses on the integration of solar PV (http://www1.eere.energy.gov/solar/rsi.html).

Solar PV is an attractive method of achieving zero-emissions energy production because it readily scales to the needed level. This makes it ideal for applications ranging from relatively small residential rooftop applications to larger commercial and industrial rooftop applications. The existing electricity infrastructure can support a limited penetration of solar PV with the current operating schemes, although the limit varies from utility to utility and feeder to feeder, depending upon the size and diversity of the load. A smart grid holds the promise of allowing much greater penetrations of solar PV and thus much greater reductions in emissions.

To estimate how much solar generation can exist in a typical residential neighborhood before reverse power flow can be expected, we examine a worst-case condition. This consists of maximum solar output at noon on a perfectly clear day, in the spring or fall when neither heating or air conditioning is needed in homes, and in a neighborhood that uses natural gas for water heating (like most U.S. homes). The electrical load at noon for a home without heating, cooling, or water heating is about $1 \mathrm{~kW}$ (Pratt et al. 1993). In this case, reverse power flow will occur when the average home in the neighborhood has a solar PV array whose output exceeds $1 \mathrm{~kW}$ on a perfectly clear day.

The daily solar energy produced by a solar PV array varies by location due to latitude and cloud cover. To estimate the annual energy production of a 1-kW solar PV array, we begin with the annual average annual average incident solar radiation on a south-facing surface (with an optimal tilt, equal to the latitude), indicated for various locations in the United States by the color coding on the map in Figure 3.9. The annual energy produced by a $1-\mathrm{kW}$ array is directly proportional to the average incident solar radiation, as described in Attachment 1, Mechanism $\mathrm{H}$. This production is expressed as the solar fraction of the annual energy requirement of such a home that is supplied by the solar PV array. (i.e., the annual solar energy produced divided by the annual electricity consumed by the home $(8600 \mathrm{kWh} / \mathrm{year}$, including air conditioning; see Attachment 1, Mechanism $\mathrm{H}$ ). The solar fraction is equivalent to the local's RPS from solar resources. 


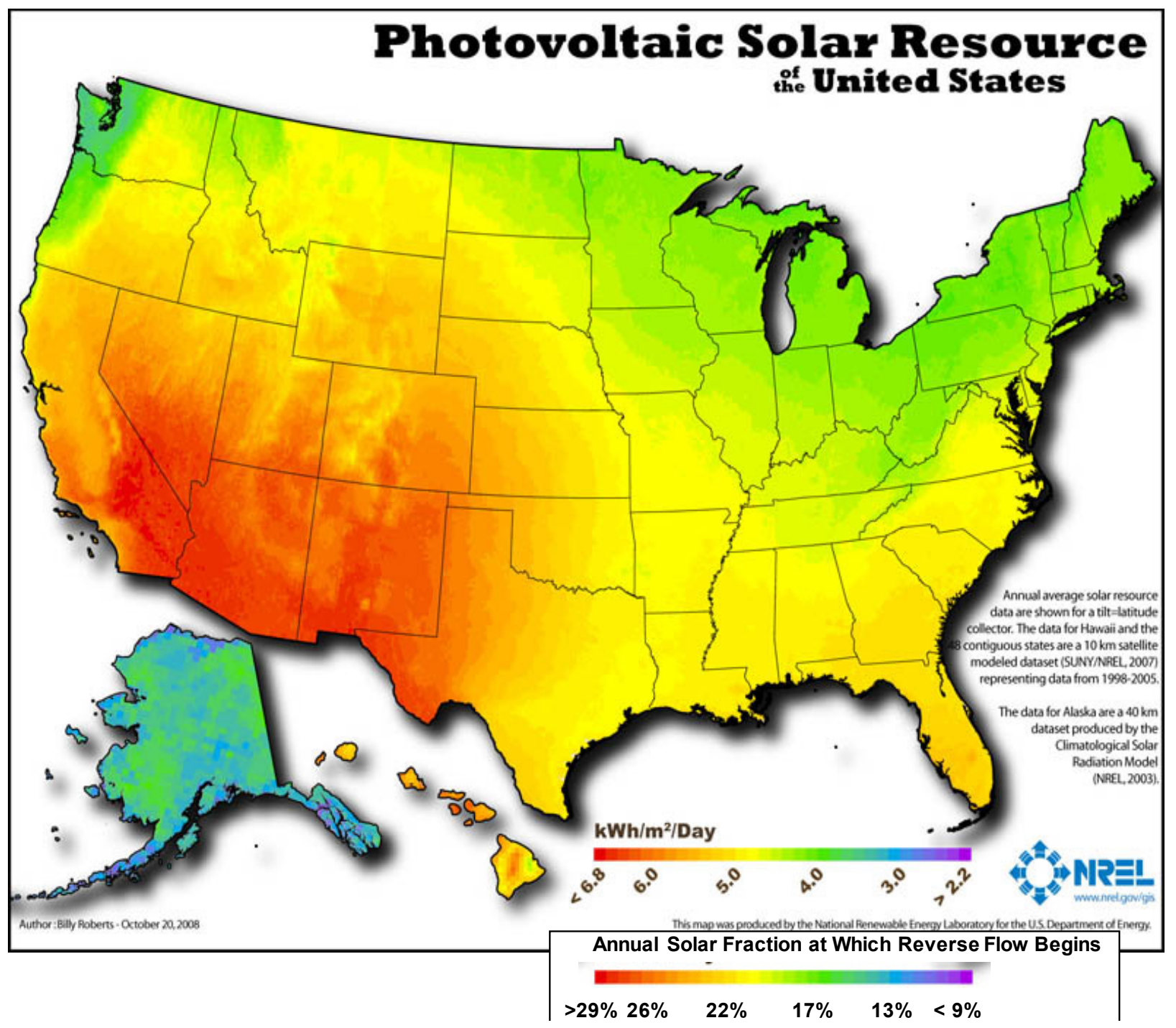

Figure 3.9. Annual Fraction of Energy from Residential Solar PV at Which Reverse Power Flow Begins

To indicate the maximum solar fraction before reverse power flow begins to occur, we have added a second scaling key to the color coding in Figure 3.9. The annual fraction of energy generated from such a $1-\mathrm{kW}$ solar array is seen to range from a low of about $17 \%$ to a high of about $28 \%$ over most of the United States (excluding Alaska and parts of the Pacific Northwest). The median for the continental United States appears to be around 21\%. If additional solar PV capacity is installed beyond the amount to supply this solar fraction, reverse power flow occurs because the output of the solar arrays on a clear day exceeds the 1-kW average load of each home.

Thus, the onset of reverse power flow appears to be a serious barrier to penetrations of solar PV systems in residential neighborhoods to achieve local RPS levels above about $20 \%$. A smart grid could help circumvent this barrier by deploying and controlling additional voltage regulators and batteries, and by providing short-circuit protection schemes that adapt to on-the-fly reverse power flow. Further details are provided in Attachment 1, Mechanism $\mathrm{H}$. Estimates of potential reductions in electricity and $\mathrm{CO}_{2}$ emissions were not made for this mechanism, and there is not an obvious basis for estimating the indirect 
benefits of removing a barrier such as this. Further refinement of this crude estimate and creating a way to value it is a recommendation for further analysis.

\subsubsection{Support Penetration of Renewable Wind Generation}

This mechanism estimates the impacts of a smart grid in helping to mitigate challenges for integrating wind energy into the electric system. The contribution of electricity generated by wind turbines is increasing due to a combination of the improved economic competitiveness of wind power, state and federal tax credits, state renewable energy portfolio requirements, and consumer desire to purchase "green" electricity. However, the integration of wind energy poses challenges because of the unpredictability and steep ramp rates of wind resources, which must be compensated by the use of more traditional power plants (termed load following or regulation) that increase costs because of redundancy and maintenance to correct increased wear and tear. Smart grid technologies, primarily communication and control over demand response, and distributed generation and storage resources, can help replace fossil fuel capacity used to overcome the unpredictability and ramping issues, and thereby increase the level of wind generation into the electric system (Todd et al. 2009).

Wind energy has benefitted greatly from RPSs and tax credits, but it is characterized by intermittency and ramping that requires additional capacity to provide ancillary services in the form of regulation, load following, and scheduling. As discussed in Attachment 1, Mechanism H, a review of efforts to estimate this impact indicates that the electrical system can accommodate penetrations of wind energy on the order of $20 \%$ to $25 \%$ with only modest increases in the requirements for ancillary services (NWPPC 2007; CAISO 2007). These studies also indicate that wind integration is facilitated in cases where the service area is geographically large and has a diversity of loads. The contribution of the smart grid technology can replace the additional capacity used to provide ancillary services with smart grid assets by using advanced communication and control technologies.

The estimated potential reductions in electricity and $\mathrm{CO}_{2}$ emissions attributable to implementation of smart grid technologies are based on the literature review and the author's experience. The direct impact of saving the fuel used for power plants offering regulation service is estimated based on the analysis in Parsons et al. (2006). Extra fuel is used for power plants because of the inefficiencies of continually changing their output. Parsons et al. (2006) estimated that the regulation requirement increases from $0.65 \%$ in the base case to $0.75 \%$ at the RPS level of $25 \%$, for a four-utility combined balancing area with a peak load of about 21,000 MW.

We estimate that the fuel consumption for a power plant supplying regulation is $20 \%$ higher than it is in steady-state operation (with a range of $10 \%$ to $30 \%$ ). Saving this fraction of the energy for the additional regulation required to achieve an RPS of $20 \%, 0.1 \%$ of total U.S. consumption, would result in saving the energy equivalent of $0.02 \%$ of U.S. electricity energy consumption and associated carbon emissions, as shown in Table 3.11. Note that $100 \%$ of the fuel used by such power plants is not saved, because they are supplying energy in addition to regulation. Rather, they waste fuel by moving their output up and down around their average operating points. 
Table 3.11. Estimated Direct Impacts of Reduced Energy Needed to Supply Regulation for Wind Energy Penetration at 25\% RPS

\begin{tabular}{|c|c|c|c|c|c|c|c|c|}
\hline \multirow[b]{3}{*}{$\begin{array}{c}\text { Est. } \\
\%\end{array}$} & \multicolumn{4}{|c|}{ Reduced Energy Consumption (2030) } & \multicolumn{4}{|c|}{ Electric Sector Annual Reductions (2030) } \\
\hline & \multirow[b]{2}{*}{$\begin{array}{c}\text { Low } \\
\%\end{array}$} & \multirow[b]{2}{*}{$\begin{array}{l}\text { High } \\
\%\end{array}$} & \multicolumn{2}{|c|}{ Baseline Electricity Consumption } & \multicolumn{2}{|c|}{ Energy } & \multicolumn{2}{|c|}{ Carbon Emissions } \\
\hline & & & End-Use Sector(s) & $\left(10^{9} \mathrm{kWh} /\right.$ year $)$ & $\begin{array}{c}\% \text { of } \\
\text { United } \\
\text { States }\end{array}$ & $\begin{array}{c}\left(10^{9} \mathrm{kWh} /\right. \\
\text { year })\end{array}$ & $\begin{array}{c}\% \text { of } \\
\text { United } \\
\text { States }\end{array}$ & $\begin{array}{l}(\mathrm{MMT} / \\
\text { yearr })\end{array}$ \\
\hline 20 & 10 & 30 & $\begin{array}{l}\text { Fuel Savings for } 0.1 \% \text { Additional } \\
\text { Regulation Requirement }\end{array}$ & 5 & 0.02 & 1 & 0.02 & 1 \\
\hline
\end{tabular}

This mechanism appears to have a negligible effect on energy and emissions because of the small amount of energy involved, although in certain areas of the country where regulation is monetized in wholesale markets, it is apparent that it is quite expensive to provide. It should be investigated as an indirect savings mechanism in future work, to reflect the potential of reduction in regulation costs.

It is worth noting that only a very small amount of load (approximately $0.7 \%$ ) would need to participate in providing all the regulation needed by the grid, as would similarly small amounts of energy from storage or batteries.

We also estimate the potential indirect reductions from reinvesting the capital cost savings from avoiding the construction of extra generation capacity required for the total operating reserves (regulation, spinning, non-spinning, load following, and reserve margin) to support wind generation to meet. This has been estimated to increase from 5\% of peak load capacity to $7 \%$ for an RPS of $25 \%$ (Smith et al. 2007).

We use the $2 \%$ difference to estimate the reduced reserve capacity, with a range from $1 \%$ to $3 \%$. We assume the resulting cost savings, from avoiding over 1000 gigawatts of power plant capacity at $\$ 1000 / \mathrm{kW}$, are reinvested in additional cost-effective energy efficiency or renewable generation at a levelized cost of $8.8 \notin / \mathrm{kWh}$. This results in very substantial estimated reductions of $5 \%$ of U.S. electricity consumption and associated $\mathrm{CO}_{2}$ emissions, as shown in Table 3.12.

Table 3.12. Estimated Indirect Impacts of Reduced Needed Reserve Capacity for Wind Energy Penetration at $25 \%$ RPS

\begin{tabular}{|c|c|c|c|c|c|c|c|c|c|}
\hline \multicolumn{6}{|c|}{ Avoided Expenditure Reinvested to Save Carbon (2030) } & \multicolumn{4}{|c|}{ Electric Sector Annual Reductions (2030) } \\
\hline \multirow[b]{2}{*}{$\begin{array}{c}\text { Est. } \\
\%\end{array}$} & \multirow[b]{2}{*}{$\begin{array}{c}\text { Low } \\
\%\end{array}$} & \multirow[b]{2}{*}{$\underset{\%}{\mathrm{High}}$} & \multicolumn{2}{|l|}{ Baseline Captial Expenditure } & \multirow[b]{2}{*}{$\begin{array}{l}\text { Savings } \\
\left(10^{9} \$\right)\end{array}$} & \multicolumn{2}{|c|}{ Energy } & \multicolumn{2}{|c|}{ Carbon Emissions } \\
\hline & & & Investment & $\left(10^{9} \$\right)$ & & $\begin{array}{c}\% \text { of } \\
\text { United } \\
\text { States }\end{array}$ & $\begin{array}{c}\left(10^{9} \mathrm{kWh} /\right. \\
\text { year })\end{array}$ & $\begin{array}{c}\% \text { of } \\
\text { United } \\
\text { States }\end{array}$ & $\begin{array}{c}\text { (MMT/ } \\
\text { year) }\end{array}$ \\
\hline 2 & 1 & 3 & $\begin{array}{l}1111 \mathrm{GW} \text { Total Generation Capacity } \\
\text { @ } \$ 1000 / \mathrm{kW}\end{array}$ & 1111 & 22 & 5 & 253 & 5 & 150 \\
\hline
\end{tabular}





\subsection{Comparison with Related Studies}

Section 4.1 of this chapter presents a summary/review of the EPRI, The Climate Group, and Hledik assessments. Section 4.2 provides a comparison of these three and PNNL assessments.

\subsection{Review of Related Studies}

\subsubsection{Electric Power Research Institute Green Grid Study}

The EPRI report examined seven topic areas for which the smart grid can provide reductions in energy consumption and/or $\mathrm{CO}_{2}$ emissions. Five of the topics were categorized as directly contributing to utility goals and provided reductions in both energy consumption and $\mathrm{CO}_{2}$ emissions. The remaining two were not categorized as directly contributing to electric utility goals, and provided a reduction in $\mathrm{CO}_{2}$ emissions. Estimates of the reductions in electricity and $\mathrm{CO}_{2}$ emissions for the seven topic areas for the year 2030 are summarized in Table 4.1 .

Table 4.1. EPRI Report: Smart Grid Energy Savings and Avoided $\mathrm{CO}_{2}$ Emissions Summary (2030).

\begin{tabular}{|c|c|c|c|c|}
\hline \multirow{2}{*}{$\begin{array}{l}\text { Emissions-Reduction Mechanism } \\
\text { Enabled by Smart Grid }\end{array}$} & \multicolumn{2}{|c|}{$\begin{array}{l}\text { Energy Savings, } 2030 \\
\text { (billion kWh) }\end{array}$} & \multicolumn{2}{|c|}{$\begin{array}{l}\text { Avoided } \mathrm{CO}_{2} \text { Emissions, } 2030 \\
\left.\text { ( } \mathrm{Tg} \mathrm{CO}_{2}\right)\end{array}$} \\
\hline & Low & High & Low & High \\
\hline $\begin{array}{l}\text { Continuous Commissioning of } \\
\text { Large Commercial Buildings }\end{array}$ & 2 & 9 & 1 & 5 \\
\hline $\begin{array}{l}\text { Reduced Line Losses (Voltage } \\
\text { Control) }\end{array}$ & 4 & 28 & 2 & 16 \\
\hline $\begin{array}{l}\text { Energy Savings Corresponding } \\
\text { to Peak Load Management }\end{array}$ & 0 & 4 & 0 & 2 \\
\hline $\begin{array}{l}\text { Direct Feedback on Energy } \\
\text { Usage }\end{array}$ & 40 & 121 & 22 & 68 \\
\hline 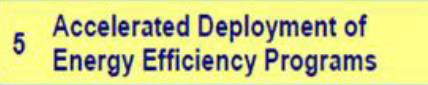 & 10 & 41 & 6 & 23 \\
\hline $\begin{array}{l}\text { Greater Integration of } \\
\text { Renewables }\end{array}$ & -. & - & 19 & 37 \\
\hline $\begin{array}{l}7 \text { Facilitation of Plug-in Hybrid } \\
\text { Electric Vehicles (PHEVs) }\end{array}$ & -. & -. & 10 & 60 \\
\hline Total & 56 & 203 & 60 & 211 \\
\hline
\end{tabular}

Source: EPRI 2008

Note: Tg equals million metric tonnes (MMT)

The estimated reductions were drawn from information contained in the literature combined with expert insight to approximate the quantity of “... the energy savings and carbon reduction impact of selected discrete mechanisms to provide insight into the magnitude of smart grid environmental benefits (EPRI 2008)." A brief description of the mechanisms addressed in the study is provided here:

- Continuous Commissioning of Large Commercial Buildings provides monitoring of equipment "health" and energy consumption performance of large commercial buildings, with notification sent 
to the energy manager in cases of performance issues. The estimate is based on its application to large commercial buildings ( $>100,000 \mathrm{ft}^{2}$ in floor area) with the provision of electricity savings of $9 \%$ and market penetration ranging from $5 \%$ to $20 \%$.

- Reducing Line Losses through voltage control and compensation for reactive power and line drop. The estimate is based on application of voltage control to the residential sector with voltage reduction of $1 \%$ to $4 \%$ and market penetration of $25 \%$ to $50 \%$.

- Energy Savings Corresponding to Peak Load Management achieved through demand response and load control programs that utilize dynamic prices and automated demand response technologies. The estimate is based on other studies, results of field work and expert judgment.

- Direct Feedback on Energy Usage to consumers through advanced meters and display devices. The estimate is based on energy savings of $2.5 \%$ in the commercial and industrial sector, and $5 \%$ in the residential sectors with market penetration of $25 \%$ to $75 \%$ in all three sectors.

- Accelerated Deployment of Energy Efficiency Programs through M\&V to reduce uncertainties regarding the performance and cost-effectiveness of energy efficiency measures, thereby increasing their deployment. The estimate is based on estimates of the maximum and realistic achievable levels of savings and expert judgment.

- Greater Integration of Renewables through communication and control strategies to compensate for temporal and intermittency factors. The estimate is based on the estimated fraction intermittency that the smart grid will resolve $(12.5 \%$ to $25 \%)$ for the integration of electricity generated by an additional $50 \mathrm{MW}$ of wind capacity in 2030. The reductions in $\mathrm{CO}_{2}$ emissions are based upon EPRI's estimated generation profile for 2030, which provides $\mathrm{CO}_{2}$ emissions of about $12 \%$, slightly lower than the emissions based on the 2006 generation profile.

- Facilitation of PHEVS through price signals and behavior modification to encourage overnight charging, thereby improving the system load factor and utilization of base-load generation resources. The estimate is based on other studies and expert judgment.

\subsubsection{Climate Group/Information and Control Technologies Report}

One focus of the Climate Group report (often referred to as the ICT report) examined reductions in $\mathrm{CO}_{2}$ emissions in four sectors (smart grid, road transportation, buildings, and travel substitution) that could be enabled by information and control technologies (ICT). This section provides the reduction in $\mathrm{CO}_{2}$ emissions for the three topic areas in the smart grid sector. The buildings sector topic is not included in this discussion because the reduction is achievable without connection to the smart grid, although the ICT enable interaction with the smart grid, which may help ensure or further increase the estimated buildings' sector reductions.

Estimates of the reductions in energy consumption and $\mathrm{CO}_{2}$ savings for the three topic areas for the year 2020 are summarized in Table 4.2. The estimates are based upon literature review and expert judgment, although the assumptions and analytical methodology underlying the estimates are not clearly stated. 
Table 4.2. Climate Group Report: Smart Grid Energy Savings and Avoided $\mathrm{CO}_{2}$ Emissions Summary (2020)

\begin{tabular}{lcc}
\hline \multicolumn{1}{c}{ Topic } & $\begin{array}{c}\text { Energy Savings, 2020 } \\
(\text { TWh })\end{array}$ & $\begin{array}{c}\mathrm{CO}_{2} \text { Emission Reductions, }{ }^{(\mathrm{a})} 2020 \\
\text { (MMT) }\end{array}$ \\
\hline Smart Grid & $162-365$ & $230-480$ \\
Integrate Renewable Resources & -- & $130-260$ \\
$\begin{array}{l}\text { Reduce Transmission \& Distribution (T\&D) } \\
\text { Losses }\end{array}$ & $104-195$ & $60-120$ \\
Real-Time Pricing & $58-170$ & $40-100$ \\
\hline
\end{tabular}

(a) Based on power sector $\mathrm{CO}_{2}$ emissions of $2630 \mathrm{MMT}$.

A brief description of the topics:

- Integrate Renewable Resources through monitoring, communication, and control strategies to compensate for temporal and intermittency factors. The estimate is based on expert recommendations and President Obama's energy plan that renewables account for $10 \%$ to $25 \%$ of generating capacity.

- Reduce T\&D Losses through voltage control and performance monitoring of grid components.

- Real-Time Pricing through communication of real-time prices to raise consumer awareness and integration of price signals with thermostats and appliances.

A second focus of the report was on policy mechanisms that could be used to facilitate and overcome the technical, economic, and behavioral barriers to implementing smart grid technologies. Policy thrusts need to address the business case for smart grid investments and to conduct demonstrations in federal utilities to stimulate private-sector smart grid efforts (Figure 4.1).

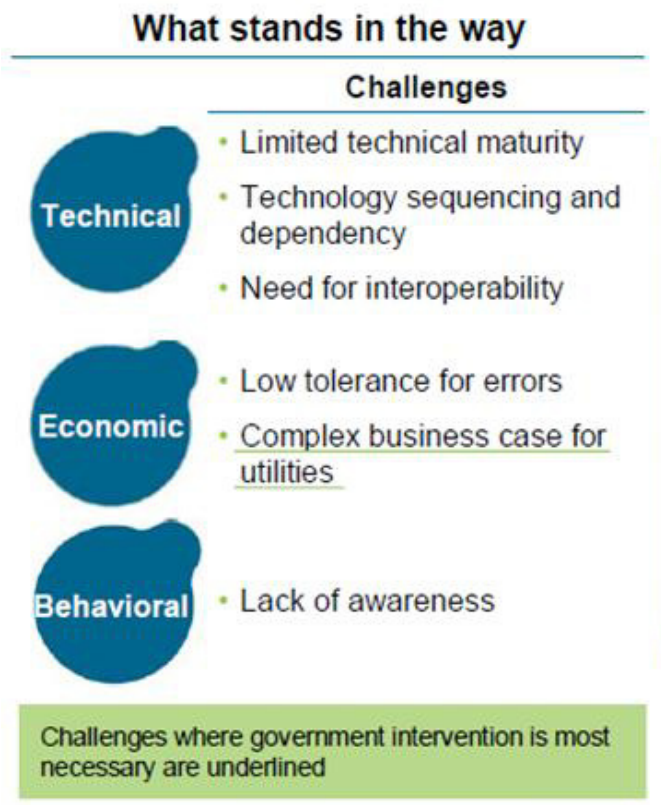

\begin{tabular}{|c|c|}
\hline \multicolumn{2}{|c|}{ What should happen } \\
\hline Policy & Key considerations \\
\hline $\begin{array}{l}\text { Provide } \\
\text { incentives for } \\
\text { utilities to } \\
\text { invest in energy } \\
\text { efficiency }\end{array}$ & $\begin{array}{l}\text { - Incentives for demand } \\
\text { reduction are most critical } \\
\text { - Adoption of smart meters } \\
\text { can help address technology } \\
\text { sequencing issues } \\
\text { - Promote open competition } \\
\text { and encourage partnerships }\end{array}$ \\
\hline $\begin{array}{l}\text { Lead by } \\
\text { example with } \\
\text { federally owned } \\
\text { utilities }\end{array}$ & $\begin{array}{l}\text { - Capitalize on strong } \\
\text { signaling effect } \\
\text { - Strive for scale to drive } \\
\text { down costs } \\
\text { - Publicize benefits and the } \\
\text { lessons from experience to } \\
\text { encourage other adopters }\end{array}$ \\
\hline
\end{tabular}

Figure 4.1. Climate Group Report: Policy Recommendations. Source: Climate Group Report 


\subsubsection{Hledik Article: How Green is the Smart Grid?}

The paper "How Green Is the Smart Grid?" examined the energy and $\mathrm{CO}_{2}$ impacts for two illustrative scenarios depicted in Figure 4.2 for the implementation of smart grid technologies in 2030, which are not intended to bracket the range of achievable reductions. The first is a "Conservative" scenario that uses cost-effective commercially available technologies (dynamic pricing, automating technologies, and information displays) in conjunction with the AMI. The second is an "Expanded" scenario that adds longer-term smart grid impacts obtained from distribution systems through increased penetration of renewable and distributed storage technologies. The scenarios were intended to examine two possibilities and were not intended as predictions of the future state.

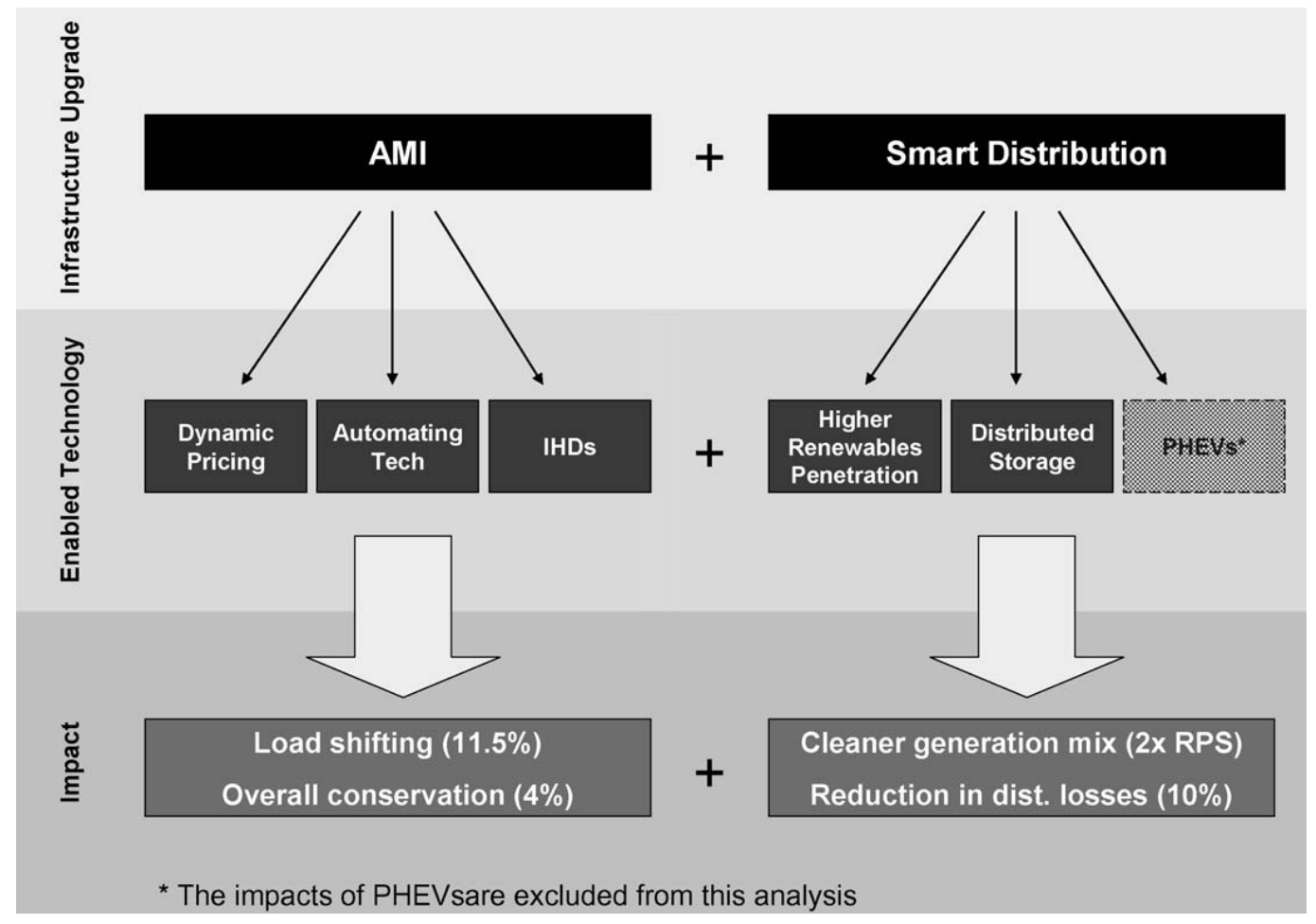

Figure 4.2. How Green is the Smart Grid? Conservative (Left Side) and Expanded Scenarios (Source: Hledik 2009)

The conservative scenario is based on an earlier analysis and the expanded scenario was based on the Regional Capacity Planning (RECAP) model that provides the least-cost mix of system generating resources for a given demand forecast. The use of the RECAP model enables differences in the regional the mix of generating resources and emission rates to be accounted for more accurately. The assumptions for the RECAP portion of the analysis are keyed to AEO 2008, and the magnitude of the energy and $\mathrm{CO}_{2}$ outputs are comparable.

Estimates of the reductions in $\mathrm{CO}_{2}$ for the topics in the conservative and expanded scenarios for the year 2030 are summarized in Table 4.3. The analysis assumes that the smart grid allows the penetration of renewable resources to double over the approximate 20\% RPS level, but it is not apparent if the reduction in $\mathrm{CO}_{2}$ emissions is for the entire penetration of $\sim 40 \%$ or only the additional $\sim 20 \%$. This is mentioned, because the reduction in $\mathrm{CO}_{2}$ emissions from increased renewable penetration is over $60 \%$ of 
the total at the $40 \%$ penetration level, and they are still the largest category at nearly $50 \%$ of the total at the $20 \%$ penetration level.

Table 4.3. Smart Grid Energy Savings and Avoided $\mathrm{CO}_{2}$ Emissions Summary (2030)

\begin{tabular}{lcc}
\hline \multicolumn{1}{c}{ Topic } & $\begin{array}{c}\text { Energy Savings, } 2030 \\
(\mathrm{TWh})\end{array}$ & $\begin{array}{c}\mathrm{CO}_{2} \text { Emission Reductions, } \\
\text { (a) } 2030\end{array}$ \\
\hline $\begin{array}{l}\text { Dynamic Pricing with Automation } \\
\text { (Load Shifting) }\end{array}$ & Conservative Scenario -- Based on AMI \\
$\begin{array}{l}\text { Dynamic Pricing with Automation } \\
\text { (Energy Efficiency) }\end{array}$ & NA & 3 \\
\begin{tabular}{l} 
In-Home Displays \\
\multicolumn{1}{c}{ Expanded Scenario - Conservative Scenario plus Increased Renewables and Storage }
\end{tabular} \\
$\begin{array}{l}\text { Integrated Renewable and Storage } \\
\text { (Cleaner Generation) }\end{array}$ \\
$\begin{array}{l}\text { Integrated Renewable and Storage } \\
\text { (Reduced Losses) }\end{array}$ & NA & 297 \\
\hline
\end{tabular}

(a) Based on power sector emissions of $3000 \mathrm{MMT}$

A brief description of the topics:

- Dynamic Pricing with Automation (Load Shifting) provides the reduction in peak load capacity through dynamic pricing with automating technologies.

- Dynamic Pricing with Automation (Energy Efficiency) provides the reduction in energy consumption through dynamic pricing with automating technologies.

- In-Home Displays provide the reduction in energy consumption through physical displays of the dynamic pricing.

- Integrated Renewable and Storage (Cleaner Generation) provides the effect of using lower-emission renewable generating and associated storage technologies.

- Integrated Renewable and Storage (Reduced Losses) provides the reduced losses due to the use of distributed renewable generating and associated storage technologies.

\subsection{Comparison}

A comparison of the three studies is difficult at best due to differences in number and definition/scope of the topics, analysis assumptions and methods, baseline data, and the time periods used to convey the reductions. The percentage reductions shown in Table 4.4 help remove the temporal component, but the above differences among the individual mechanisms examined in the four studies still makes a strict comparison tenuous. 
Table 4.4. Comparison of Estimated Reductions in Energy Consumption and $\mathrm{CO}_{2}$ Emissions

\begin{tabular}{|c|c|c|c|}
\hline \multirow[b]{2}{*}{ Mechanism } & \multicolumn{2}{|c|}{$\begin{array}{c}\text { Reduced Energy Consumption } \\
\text { and } \mathrm{CO}_{2} \text { Emissions, } \%\end{array}$} & \multirow[t]{2}{*}{ Comment } \\
\hline & Direct & Indirect & \\
\hline \multicolumn{4}{|c|}{ PNNL } \\
\hline $\begin{array}{l}\text { Conservation Effect of Consumer Information and } \\
\text { Feedback Systems }\end{array}$ & 3 & -- & \multirow{9}{*}{$\begin{array}{l}\text { Relative to } \\
\text { DOE/EIA } 2030 \\
\text { Reference Case }\end{array}$} \\
\hline $\begin{array}{l}\text { Joint Marketing of Energy Efficiency and Demand } \\
\text { Response Programs }\end{array}$ & -- & 0 & \\
\hline $\begin{array}{l}\text { Deployment of Diagnostics in Residential and } \\
\text { Small/Medium Commercial Buildings }\end{array}$ & 3 & -- & \\
\hline M\&V for Energy Efficiency Programs & 1 & 0.5 & \\
\hline Shifting Load to More Efficient Generation & $<0.1 \%$ & -- & \\
\hline Support Additional EVs and PHEV & 3 & -- & \\
\hline $\begin{array}{l}\text { Conservation Voltage Reduction and Advanced } \\
\text { Voltage Control }\end{array}$ & 2 & -- & \\
\hline $\begin{array}{l}\text { Support Penetration of Renewable Wind Generation, } \\
25 \% \text { RPS (assumed similar for solar PV) }\end{array}$ & $<0.1$ & 5 & \\
\hline Total & 12 & 6 & \\
\hline \multicolumn{4}{|c|}{ EPRI } \\
\hline $\begin{array}{l}\text { Continuous Commissioning of Large Commercial } \\
\text { Buildings }\end{array}$ & 0.04 to 0.2 & 0.03 to 0.2 & \multirow{8}{*}{$\begin{array}{l}\text { Relative to } \\
\text { DOE/EIA } 2030 \\
\text { Reference Case }\end{array}$} \\
\hline Reducing Line Losses & 0.1 to 0.6 & 0.07 to 0.5 & \\
\hline $\begin{array}{l}\text { Energy Savings Corresponding to Peak Load } \\
\text { Management }\end{array}$ & 0 to 0.1 & 0 to 0.07 & \\
\hline Direct Feedback on Energy Usage & 0.8 to 2.4 & 0.8 to 2.3 & \\
\hline $\begin{array}{l}\text { Accelerated Deployment of Energy Efficiency } \\
\text { Programs }\end{array}$ & 0.2 to 0.8 & 0.2 to 0.8 & \\
\hline Greater Integration of Renewables & -- & 0.6 to 1.3 & \\
\hline Facilitation of PHEVs & -- & 0.3 to 2.0 & \\
\hline Total & 1.1 to 4.1 & 2.0 to 7.2 & \\
\hline \multicolumn{4}{|c|}{ Climate Group } \\
\hline Integrate Renewable Resources & -- & -4.9 to $-9.9 \%$ & \multirow{4}{*}{$\begin{array}{l}\text { Relative to } 2020 \\
\text { DOE/EIA } 2020 \\
\text { Reference Case }\end{array}$} \\
\hline Reduce T\&D Losses & 2.3 to -4.3 & -2.3 to -4.6 & \\
\hline Real-Time Pricing & -1.3 to -3.8 & -1.5 to -3.8 & \\
\hline Total & 3.6 to 8.1 & 8.7 to 18.3 & \\
\hline
\end{tabular}


Table 4.4. (contd)

\begin{tabular}{|c|c|c|c|}
\hline Mechanism & $\begin{array}{l}\text { Reduced Energy } \\
\text { Consumption }\end{array}$ & $\begin{array}{l}\text { Reduced } \mathrm{CO}_{2} \\
\text { Emissions, \% }\end{array}$ & Comment \\
\hline \multicolumn{4}{|c|}{ Hledik } \\
\hline Dynamic Pricing with Automation (Load Shifting) & \multirow{2}{*}{$\begin{array}{l}11.5 \% \\
\text { reduction in } \\
\text { capacity }\end{array}$} & 0.1 & \multirow{6}{*}{$\begin{array}{l}\mathrm{CO}_{2} \text { Emissions } \\
\text { based on } 2030 \\
\text { power sector } \\
\text { emissions of } \\
3000 \text { MMT, } \\
\text { which is } \\
\text { approximately } \\
\text { the DOE/EIA } \\
2030 \text { reference } \\
\text { case }\end{array}$} \\
\hline Dynamic Pricing with Automation (Energy Efficiency) & & 3.3 & \\
\hline In-Home Displays & $\begin{array}{l}4 \% \text { reduction in } \\
\text { energy }\end{array}$ & 1.7 & \\
\hline $\begin{array}{l}\text { Integrated Renewable and Storage (Cleaner } \\
\text { Generation) }\end{array}$ & \multirow{2}{*}{$\begin{array}{l}10 \% \text { reduction } \\
\text { in distribution } \\
\text { losses }\end{array}$} & 9.7 & \\
\hline Integrated Renewable and Storage (Reduced Losses) & & 0.7 & \\
\hline Total & & 5.1 to 15.7 & \\
\hline
\end{tabular}

Note: The base from the percentages is forecasted net generation and total energy-related $\mathrm{CO}_{2}$ emissions from the AEO 2008 (Tables A8 total sector electricity sales, and A18 total emissions, the estimates (i.e., numerators) in Tables 4.1, 4.2, and 4.3 were also based on the AEO 2008 forecasts, with slight modifications in some cases. 



\subsection{Recommendations and Issues}

This section provides a summary of issues identified for each of the nine mechanisms and recommendations for addressing these issues. A more detailed discussion of each of the mechanisms, with recommendations, is contained in Attachment 1. In addition, a number of additional issues that may impact the penetration of smart grid technologies are presented.

\subsection{Mechanism Recommendations}

\subsubsection{Conservation Effect of Consumer Information and Feedback Systems}

This discussion provides recommendations to improve the understanding and effectiveness of feedback that makes consumers aware of their energy consumption and modifications they can make to behaviors in response to information and price signals. This feedback process is necessary to obtain the mutual benefits to customers and utilities from energy efficiency, demand response, diagnostics, and other programs that require interaction with and response from customers. A review of these mechanisms, with a complete list of recommendations for improving the design and implementation of feedback, is presented in Attachment 1.

Four methodological issues were identified in the review. The primary issue is the extent to which results are influenced by self-selection of sample respondents, because they may be more motivated with higher levels of environmental concerns and self-efficacy. A second methodological issue is that very few studies evaluated the persistence of the conservation and efficiency effects observed to ensure that behavior change is maintained and rebound effects are prevented. A third methodological issue concerns the sample size and variability of the studies, as in the studies reviewed; the samples were limited to no more than several hundred households that were as homogeneous as possible. The final methodological issue relates to the behavioral granularity of the conservation and efficiency effects reported to understand how the savings obtained from a program were actually achieved.

Separate recommendations were formulated to address each of these issues, with a common theme that called a large and diverse sample tracked over a long period of time (24 months), with a study tailored to examine the respective issue. An alternative would be to segment the large sample to examine the behavioral issues in parallel to develop a more robust feedback system. In either case, the examination should include additional issues pertinent to consumer behavior to better understand savings effects and behavioral "entry points" for various groups of consumers, as outlined in the review in Attachment 1.

\subsubsection{Deployment of Diagnostics in Residential and Small/Medium Commercial Buildings}

The issue is that many technologies are required to enable diagnostics in buildings to be performed through the smart grid. Some of these are being developed and marketed today; others are missing from the marketplace. The recommendation is to pursue development of analytic software-based technologies that either are needed for, or could contribute to, cost-effective automated energy management. They include those that: enhance and system operation in residential and small commercial buildings, automate 
fault detection and diagnostics, automate commissioning, enable price-based controls, and enable coordination and integration with other systems.

\subsubsection{Joint Marketing of Energy Efficiency and Demand Response Programs}

The issue is that customers are often confused by the differentiation between energy efficiency and demand response programs; combining the administration of these two types of programs would improve their effectiveness by eliminating the confusion and provide cost savings that could be reinvested in energy efficiency programs. This issue is not addressed in the literature and program data is not in the form that permits estimation of administrative cost reductions or increases in program effectiveness. The recommendation is to assess the impact of co-administering energy efficiency and demand response programs to determine the magnitude of reductions in administrative costs, energy efficiency, and demand response when the programs are administered together. This would enable extrapolation of how the implementation of smart grid technologies may influence the effectiveness (e.g., energy, capacity, and utility cost) of merging energy efficiency and demand response administrative/delivery structures.

\subsubsection{Measurement \& Verification for Energy Efficiency Programs}

The issue is that $\mathrm{M} \& \mathrm{~V}$ program evaluations of program effectiveness and technology performance are often limited by budget constraints, which lead to reductions in the scope and duration of the effort, as well as the methods used, and which decreases accuracy and transparency. The recommendation is to develop software-based analytic methods that leverage the smart grid's metering and communication abilities to expand the sample size, improve data granularity, and increase the duration of M\&V efforts. This will provide for increased accuracy and transparency, lower cost, and assessment of persistence.

\subsubsection{Shifting Load to More Efficient Generation}

The issue is that estimation of reductions in energy and $\mathrm{CO}_{2}$ emissions is subject to significant uncertainty due to the types of power plants that provide base, intermediate, peak load power, and the order for dispatch. It is recommended that the estimates of energy and $\mathrm{CO}_{2}$ reductions that result from load shift be the subject of more methodical efforts to determine differences that may result by minimizing economic impacts, maximizing energy reductions, and maximizing $\mathrm{CO}_{2}$ reductions.

\subsubsection{Support Additional Electric Vehicles and Plug-In Hybrid Electric Vehicles}

The issue is that analyses to date provide considerable uncertainty for the reduction in $\mathrm{CO}_{2}$ emission due to the high dependency on the reference vehicle to which a PHEV or EV is compared, and the timing and duration of the charging influence on the carbon intensity. In addition, analyses do not provide a uniform estimate of the economic benefits of off-peak-load charging by utilizing higher efficient intermediate or base-load power plants. It is recommended to employ a more comprehensive analysis that analyzes the incremental energy, economic, and environmental benefits of load-management strategies of a growing EV fleet. 


\subsubsection{Conservation Voltage Reduction and Advanced Voltage Controls}

The issue is that the majority of current knowledge regarding the effectiveness of voltage reduction and controls is empirical, and cannot be validated analytically. Consequently, we do not fully understand how new technologies will interact with CVR and thus be fully exploited. It is recommended to conduct additional research to improve understanding about the fundamental nature of CVR and how it can be exploited to interact with demand response and distributed energy resources to provide improved system operation with energy, capacity, and emission benefits.

\subsubsection{Support Penetration of Solar Generation (Renewable Portfolio Standard $>20 \%$ )}

The issue is that the tipping point where solar PV "helps" the system to become a limiting factor that is detrimental to the system cannot be simply stated, because of the variability of the solar resource and the size and diversity of the system. Additional research is needed to determine the feasible limit with existing technologies and to determine new operating strategies based on smart grid monitoring and control capabilities that allow for greater penetration of solar PV.

\subsubsection{Support Penetration of Renewable Wind Generation (Renewable Portfolio Standard $>20 \%$ )}

The issue and recommendation is virtually identical to that for the integration of solar PV to determine the limit to integration of wind resources and the smart-grid-based operating and control strategies that consider load size and diversity, storage, and demand response to provide guidelines for implementation efforts.

\subsection{Additional Issues}

A number of issues not connected to the nine mechanisms examined in this report may impact the penetration of smart grid technologies:

- Proof of the cost effectiveness of smart grid technologies on the demand side may cause difficulty at state regulatory hearings for demand response resources (SmartGridNews.com 2009b). Related issues may be 1) the integration of smart grid elements into the IRP methodology commonly used by utilities and regulatory commissions, and 2) the inclusion of the monetized value of carbon, and 3) the inclusion of the monetized value of other emissions and impacts.

- An account of the energy and carbon savings that will accrue with the integration of solar PV and wind due to the reduction in unaccounted for parasitic loads associated with the operation of fossilfuel powered plants (these loads increase with carbon capture) and transmission of electricity.

- An account of the energy and carbon savings that will result from a decrease in reactive power and a decrease in the load on the T\&D networks .

- Development of a quantitative method to monetize improvements in power reliability and quality, and reductions in T\&D congestion that will be realized with smart grid operations.

- Even though energy and $\mathrm{CO}_{2}$ emissions typically go hand-in-hand, the smart grid may produce a greater reduction in $\mathrm{CO}_{2}$ emissions than in energy use in cases in which the load is reduced from $\mathrm{CO}_{2}$ - 
intensive peak load generating sources to less $\mathrm{CO}_{2}$-intensive intermediate or base-load power plants. This presents an interesting future where demand response and distributed generation resources can be managed on strictly economic-based criteria, strictly $\mathrm{CO}_{2}$-based criteria, or a mixture of both.

- To realize the estimated reductions the smart grid can deliver, two offsetting increases in consumption need to be accounted for. The first assumes that a server is needed in every distribution substation to monitor end-use loads, provide two-way communications with customers and, where user permitted, provide automated demand response. The number of distribution substations is unknown, so an assumption of 100,000 substations is made based upon an estimated 300 to 400 thousand feeders and 3 to 5 feeders per substation. Each server is expected to draw $1 \mathrm{kw}$ for every hour of the day throughout the year, thus increasing expected energy consumption by nearly $1 \mathrm{~B} \mathrm{kWh} /$ year. The second assumes that demand response/GFA devices are installed in the entire stock of $466 \mathrm{M}$ appliances (heat pumps, air conditioners, dryers, refrigerators, and freezers) (EIA-AEO 2008), individually draw a load of 1 to $5 \mathrm{w}$ every hour of the day throughout the year, to additionally increase expected energy consumption by 4 to $20 \mathrm{~B} \mathrm{kWh}$. The combined effect of the two offsets may increase the 2030 electric utility sector energy and $\mathrm{CO}_{2}$ emissions by $0.1 \%$ to $0.4 \%$. While the increase is small and may not be considered important, it does point to the need for technology developers to minimize the increased loads of smart grid technologies. 


\subsection{References}

Beck RW. 2007. Distribution Efficiency Initiative. Technical Report prepared for the Northwest Energy Efficiency Alliance, Portland, Oregon.

Berst J. April 13, 2009. "Four Keys to Managing Smart Grid Evolution." SmartGridNews.com. Accessed on April 14, 2009 at http://www.smartgridnews.com/artman/publish/commentary/Four_Keys to_Managing_Smart_Grid Evolution-564.html Brambley MR, S Katipamula, and P O'Neill. 2009. "Diagnostics for Monitoring-Based Commissioning." In: Proceedings of the 2009 National Conference on Building Commissioning. PNWD-SA-8612, Battelle-Pacific Northwest Division, Richland, Washington. Accessed December 29, 2009, at http://www.peci.org/ncbc/2009/docs/Brambley NCBC09P.pdf.

Boyer SA. 2007. "Collecting Data from Distant Facilities." InTech. Accessed December 29, 2009, at http://www.isa.org/InTechTemplate.cfm?Section=Control_Fundamentals1\&template=/ContentManag ement/ContentDisplay.cfm\&ContentID $=64710$.

California Independent System Operator Corporation (CAISO). 2007. Integration of Renewable Resources; Transmission and operating issues and recommendations for integrating renewable resources on the California ISO-controlled Grid. CAISO, Folsom, California.

DOE - U.S. Department of Energy. 2002. National Transmission Grid Study. U.S. Department of Energy, Washington, D.C. Accessed December 29, 2009, at www.ferc.gov/industries/electric/geninfo/transmission-grid.pdf.

DOE - U.S. Department of Energy. 2008. The Smart Grid: an Introduction. Prepared for DOE by Litos Strategic Communication. Accessed December 29, 2009, at http://www.oe.energy.gov/1165.htm.

DOE - U.S. Department of Energy. 2009. Electricity 101. U.S. Department of Energy, Washington, D.C. Accessed December 29, 2009, at http://www.oe.energy.gov/information_center/electricity101.htm.

DOE/EIA - U.S. Department of Energy/U.S. Energy Information Administration. 2008. Annual Energy Outlook 2008. DOE/EIA-0383(2008). U.S. Department of Energy, Washington, D.C. Accessed December 29, 2009, at http://www.eia.doe.gov/oiaf/archive/aeo08/index.html.

DOE/EIA - U.S. Department of Energy/U.S. Energy Information Administration. 2009. Electric Power Annual. DOE/EIA-0348(2007). U.S. Department of Energy, Washington, D.C. Accessed December 29, 2009, at http://www.eia.doe.gov/cneaf/electricity/epa/epa sum.html.

DOE/FEMP 2009. U.S. Department of Energy, Federal Energy Management Program. U.S. Department of Energy, Washington, D.C. Financing and Pricing Evaluation Workshop for Federal ESPCs.

DOE/OE - U.S. Department of Energy, Office of Electricity Delivery and Energy Reliability. 2006. Fact Sheet: The Electricity Delivery System. U.S. Department of Energy, Washington, D.C. Accessed December 29, 2009, http://sites.energetics.com/gridworks/pdfs/factsheet.pdf. 
Drenker S and A Kader. 1999. "Nonintrusive Monitoring of Electrical Loads." IEEE Computer Applications in Power 12(4):47-51.

Electric Advisory Committee (EAC). 2008. Smart Grid: Enabler of the New Energy Economy. U.S. Department of Energy, Washington, D.C. Accessed December 29, 2009, at www.oe.energy.gov/DocumentsandMedia/final-smart-grid-report.pdf.

Efficiency Valuation Organization (EVO). 2007. International Performance Measurement and Verification Protocol: Concepts and Options for Determining Energy and Water Savings, Volume 1. EVO 1000-1.2007, EVO, San Francisco, California.

EPA - U.S. Environmental Protection Agency. 2008. National Action Plan for Energy Efficiency Vision for 2025: A Framework for Change. Accessed on December 29, 2009, at http:/www.epa.gov/RDEE/energy-programs/napee/resources/vision2025.html.Electric Power Research Institute (EPRI). 2008. The Green Grid: Energy Savings and Carbon Emissions Reductions Enabled by a Smart Grid. 1016905, EPRI, Palo Alto, California. Accessed December 29, 2009 , at http://my.epri.com/portal/server.pt?space $=$ CommunityPage \&cached $=$ true \&parentname $=$ ObjMgr\&par entid $=2 \&$ control $=$ SetCommunity $\&$ Community $I D=404 \&$ RaiseDocID $=000000000001016905 \&$ Raise DocType $=$ Abstract idn.

Electric Power Research Institute (EPRI). 2009. Assessment of Achievable Potential from Energy Efficiency and Demand Response Programs in the U.S.: (2010-2030). 1016987, EPRI, Palo Alto, California. Accessed December 29, 2009, at http://mydocs.epri.com/docs/public/000000000001018363.pdf.

Faruqui A, S Sergici, and A Sharif. 2009. "The Impact of Informational Feedback on Energy Consumption - A Survey of the Experimental Evidence.” Energy (in press).

Fels M and C Reynolds. 1993. Heating-and-Cooling (HC) PRISM: Background, Sample Applications, and Validation Studies. Report for Advanced PRISM Project, Center for Energy and Environmental Studies. Princeton University, Princeton, New Jersey.

Fischer C. 2008. "Feedback on Household Electricity Consumption: A Tool for Saving Energy?" Energy Efficiency 1(1):79-104.

Global e-Sustainably Initiative (GeSI). 2008. SMART 2020: Enabling the Low Carbon Economy in the Information Age, United States Report Addendum. Prepared by Boston Consulting Group for GeSI. Accessed December 29, 2009, at http://www.smart2020.org.

Hledik R. 2009. "How Green is the Smart Grid?” The Electricity Journal 22(3):29-41.

Kintner-Meyer M, K Schneider, and R Pratt. 2007. "Impact Assessment of Plug-In Hybrid Vehicles on Electric Utilities and Regional U.S. Power Grids. Part 1: Technical Analysis." Journal of EUEC, 1:Paper \#04. http://www.euec.com/documents/pdf/Paper 4.pdf

McKinsey \& Company. 2007. Pathways to a Low-Carbon Economy. McKinsey \& Company, Washington, D.C. 
McKinsey \& Company. 2009. Unlocking Energy Efficiency in the U.S. Economy. McKinsey \& Company, Washington, D.C.

Northwest Power and Conservation Council (NWPPC). 2007. The Northwest Wind Integration Action Plan. WIF Document 2007-1, Portland, Oregon.

Pacific Northwest National Laboratory (PNNL). 2007. Pacific Northwest GridWise ${ }^{\text {TM }}$ Testbed Demonstration Projects, Part I. Olympic Peninsula Project. PNNL-17167. PacificNorthwest National Laboratory, Richland, Washington.

Parsons B, M Milligan, JC Smith, K Porter, E Demeo, B Oakleaf, K Wolf, R Zavadil, H Shiu, and DY Nakafuji. 2006. "Grid Impacts of Wind Power Variability: Recent Assessments from a Variety of Utilities in the United States.” In: European Wind Energy Conference, February 27-March 2, Athens, Greece. NREL/CP-500-39955.

Pratt, RG, CC Conner, BA Cooke, and EE Richman. 1993. "Metered End-Yse Consumption and Load Shapes from the ELCAP Residential Sample of Existing Homes in the Pacific Northwest." Energy and Buildings, Vol. 19, No. 3. Elsevier Science Publishing, New York.

Scott MJ, M Kintner-Meyer, DB Elliot, and WM Warwick. 2007. "Impact Assessment of Plug-In Hybrid Vehicles on Electric Utilities and Regional U.S. Power Grids. Part 2: Economic Assessment." Journal of EUEC 1:Paper No. 05. Accessed December 29, 2009, at http://www.euec.com/documents/pdf/Paper_5.pdf.

Smith JC, B Parsons, T Acker, M Milligan, R Zavadil, M Schuerger, and E DeMeo. 2007. "Best Practices in Grid Integration of Variable Wind Power: Summary of Recent U.S. Case Study Results and Mitigation Measures.” In: European Wind Energy Conference 2007, May, Milan, Italy. Accessed August 5, 2009, at http://www.uwig.org/opimpactsdocs.html.

Todd D, M Caufield, B Helms, M Starke, B Kirby, and J Kueck. 2009. Providing Reliability Services through Demand Response: A Preliminary Evaluation of the Demand Response Capabilities of Alcoa Inc. ORNL/TM-2008/233, Oak Ridge National Laboratory, Oak Ridge, Tennessee. 



\section{Attachment 1}

Mechanism Review and Analysis 



\section{Mechanism A: Conservation Effect of Consumer Information and Feedback Systems}

\section{A.1 Introduction}

This topic examines the behavior of residential sector consumers related to in-home energy consumption. Approximately $11 \%$ of energy use is related to direct in-home consumption for space conditioning, illumination, and appliances (Shui and Dowlatabi 2005). Many analyses suggest that reduced home energy usage by consumers can make a substantial contribution to overall energy efficiency and greenhouse gas (GHG) emission reduction (Sanquist 2008; Gardner and Stern 2008; Shui and Dowlatabi 2005). A variety of approaches to influence consumer behavior are possible, including smart grid technology and feedback systems. This topic reviews the research concerning feedback on energy use (as distinct from capacity) and its effects on residential sector consumer behavior, identifies potential quantitative benefits in terms of reduced energy use and GHG emissions, and proposes research and development directions warranting further exploration.

While the main focus of this section concerns the potential benefits of smart grid technologies, which include detailed and timely energy feedback and a variety of usage information analyses at the utility level, there are certain overriding psychological and behavioral considerations that set the context for potential benefits. Primary among these is the issue of energy invisibility, which refers to the gradual decoupling of overt human behavior from energy usage. With the transition from wood fuel to coal through gas and electric power for space conditioning, the overt actions required to obtain energy have all but been eliminated (Stern and Aronson 1984). Further, while a variety of studies have shown that consumers are influenced by incentives to reduce their energy use, this does not suggest that people routinely behave as rational decision makers. Indeed, there is ample evidence to show that people often express a belief about energy use but behave in an opposite manner (for example, weatherization decisions). This general finding applies more broadly to the value of information, i.e., simply providing information to consumers does not necessarily alter their behavior. Instead, a variety of other factors combine to influence the final outcome. It is important to realize that the enhanced information, analysis, and delivery prospects offered by smart grid technologies will be embedded in an overriding socio-economic context. governed more by behavioral momentum (inertia) than attitudes and information (Ehrhardt-Martinez 2008; Stern and Aronson 1984).

\section{A.2 Review of Information Intervention and Feedback Studies}

This section draws from several recent comprehensive reviews of behavioral studies, including Abrahamse et al. (2005); Darby (2006); Fischer (2008); and Erhardt-Martinez (2008). Abrahamse et al. (2005) evaluated studies in terms of the type of manipulation made, i.e., providing information or requesting commitments and goal setting on the part of the consumer, as well as the effects of providing feedback by various mechanisms. Darby (2006) and Fischer (2008) focus specifically on feedback, while Erhardt-Martinez (2008) provides a comprehensive socio-economic framework for behavioral approaches. The studies presented below are limited to those in which percentage savings have been reported during the intervention period or longer-term follow-up, and for which the research is based on field studies in residential settings. We focus here on positive results, i.e., studies that actually show energy savings. However, the reader should keep in mind that a substantial percentage $(22 \%)$ of the 
reviewed studies showed no energy savings, and that fewer than $10 \%$ investigated long-term effects (1 year or greater).

\section{A.2.1 Information Interventions}

Becker (1978) reported a study involving goal setting and information feedback to reduce electricity use. Households that were challenged with a difficult goal and receiving feedback three times weekly concerning energy usage saved $15.1 \%$ compared to households given an easier savings goal. The information provided related to which appliances used the most electricity. In contrast, Geller (1981) provided workshop-based information to consumers about energy saving measures and found that while intentions to save energy increased, there were no corresponding changes in energy use based on home visits. There is some evidence to suggest that self-report of energy conservation portrays a more socially desirable result than is actually the case (Luyben 1982).

Tailored information based on energy audits and interviews has been shown to reduce energy consumption. Winnet et al. (1982) found that households receiving energy audit information concerning heating and air conditioning used $21 \%$ less electricity than a control group. McMakin et al. (2002) provided targeted information related to heating in the state of Washington and air conditioning in the state of Arizona. It found that households in Washington saved 10\% during the study period compared to a baseline measure, whereas the Arizona residents used 2\% more energy. A study involving a cable TV program illustrating specific energy-saving measures obtained a $10 \%$ energy reduction compared to a control group (Winnett et al. 1985).

\section{A.2.2 Feedback on Current Energy Usage}

An early study of continuous feedback (McClelland and Cook 1979) provided feedback for a period of 11 months, with a meter showing electricity use in cents per hour. The group receiving this feedback used $12 \%$ less electricity than a control group over the 11-month test period. A similar continuous cost indicator study by Hutton et al. (1986) found a $4 \%$ to $5 \%$ savings in a Canadian city, but no savings in an American city. The Americans showed an increase in knowledge, but no behavioral effect. The frequency of feedback has been shown to influence savings, such that continuous feedback yields a $12 \%$ savings and monthly feedback yields $8 \%$, whereas an "information only" condition yields $4 \%$. All savings were calculated relative to a control group. More recently, Chassin and Kiesling (2008) showed a $20 \%$ savings in a time-of-use pricing group with continuous feedback, compared to real-time pricing and control groups. This contrasts with an earlier finding by Sexton et al. (1987), who showed that feedback resulted in shifting use from peak to non-peak-load times, but no change in overall usage.

Feedback frequency appears to influence the level of energy savings, such that daily feedback is better than weekly or longer intervening periods. For example, Winnet et al. (1979) compared daily feedback with self-monitoring based on households reading their own meters and found that daily feedback yielded a $13 \%$ savings compared to $7 \%$ in the self-monitoring group.

Comparative feedback involves presenting information to consumers about their energy consumption relative to others. Midden et al. (1983) evaluated individual versus comparative feedback and found little difference, although there were savings in the range of $6 \%$ to $19 \%$ for electricity usage. Brandon and Lewis (1999) showed that computer-based feedback is relatively more effective than leaflets, and that 
high and medium consumers save energy with feedback while low consumers increase energy use (a rebound effect). Staats et al. (2004) provided comparative feedback through EcoTeams, i.e., small groups of neighbors, friends, and family who gather monthly to exchange information and to receive information on their own energy savings and comparisons to other teams. Savings were observed during the study period, and during a two-year follow-up indicating electricity savings of $8 \%$ and gas savings of $17 \%$.

\section{A.2.3 Home Automation/Smart Metering and Advisory Systems}

Recently there have been several studies reported in which the use of the two-way communication capabilities of smart grid technology have been used to provide feedback to residential households with web-based or energy information system displays (Ueno et al. 2006). The Ueno et al. study used a load survey meter that measured electricity consumption for the entire house, and an end-use meter that provided power consumption at intervals of 30 minutes. The sample was limited to nine households because of the complexity and expense of the technology. The information display provided a variety of outputs, including daily load curves for each appliance, percentage of overall consumption, patterns of consumption over 10-day periods, and various recommendations for saving energy. The results indicated an initial high level of interaction with the display, which leveled off after 10 days, followed by a relatively constant number of interactions and responses to tips provided by the system. Overall, the households reduced consumption by $9 \%$ during the test period.

Abrahamse et al. (2007) showed that similar targeted information based on self-reported behaviors, and using a web-based tool for feedback, can result in 5\% savings (study was done on the basis of questionnaires and overall meter load). The results of both studies indicated that the process of making consumers aware of their specific energy-consuming actions in the context of conservation goals results in savings. A similar personalized advisory system reported by Benders et al. (2006) demonstrated an $8.5 \%$ reduction in consumption. Wood and Newborough (2007) suggest that applying principles of information design based on various categories of energy usage will be an effective method to portray usage and savings. With sufficient sample granularity, specific use patterns emerge that can be used as guides to conservation behavior (Firth et al. 2008).

\section{A.3 Summary of Feedback Effects}

The results described in this review are consistent with the conclusion that feedback from metered measurement of energy consumption in the home can lead to energy savings when provided under the proper circumstances. The magnitude of the energy savings impact produced by this feedback effect in the residential sector would depend on how effectively two-way communication systems could penetrate the residential market and how residents respond to the information provided by these meters. Feedback tends to be most effective when it is:

- based on actual usage data

- provided on a frequent basis (daily is better than weekly, etc.)

- involves goal setting and choice

- is provided over a long period

- involves specific behavioral recommendations regarding appliances

- involves normative or historical comparisons. 
Fischer (2008) contends that these results favor the capabilities offered by smart metering and two-way communication, which provides an effective way of engaging the consumer and providing tailored feedback. The specific energy-use reductions achieved range from $5 \%$ to $20 \%$, with a median of approximately $6 \%$ (Fischer 2008). Similar results have been observed in utility field studies reviewed by Faruqui et al. (2009). The values range widely due to substantial differences in research methodology.

The reductions in electricity and $\mathrm{CO}_{2}$ emissions calculated in Attachment 2 are based on the literature review and the author's experience. It is estimated that a direct reduction of $6 \%$ in electricity consumption, with a range of $1 \%$ to $10 \%$, can be achieved in the residential and small/medium commercial building sectors through implementation of smart grid technologies. No indirect reductions in electricity or capacity are expected.

\section{A.4 Recommendations for Additional Work and/or Consideration}

The studies reviewed above provide convincing evidence that consumers will change their energy consumption behavior in response to feedback, and that the conditions surrounding feedback, such as frequency and specificity, are influential variables. This implies that a smart grid/metering system may yield considerable savings, both in terms of end-use conservation as well as its basic goal-time-of-use load shifting. This section discusses some of the behavior-oriented research questions that need to be addressed in relation to larger-scale implementation of smart grid/metering systems to ensure that the potential savings from behavioral changes are realized, and discusses research approaches to address these questions.

There are several methodological issues raised by the research reviewed above. The primary issue is the extent to which results are influenced by self-selection of sample respondents; they would generally appear to be a very motivated group of subjects with higher levels of environmental concerns and most likely higher levels of self-efficacy. Would the conservation and efficiency effects observed in the feedback studies be observed with a broader range of participants?

A general research approach to this issue would be to implement a large-scale (several thousand households) smart-metering program in selected geographic areas, and to provide only explanatory material concerning the new equipment-which would include the potential for self-generated savings, but otherwise involve no change in rates or incentive structure. Instead, any behavioral changes would be based on "discovery" by the end users interacting with the technology, and receiving commensurate feedback on savings.

A second methodological issue pertains to the sustainability of the conservation and efficiency effects observed. Very few studies evaluated the durability of effects, and those that did found mixed results. The most encouraging result came from a Danish study using EcoTeams that provided a social component to the feedback (Stats et al. 2004), and found that conservation effects were maintained 24 months after the intervention. It is important that large-scale implementations take steps to ensure that behavior change is maintained and rebound effects are prevented. In the proposed large-scale sample described above, the first step in studying this issue would be to analyze the savings effects observed from metering on a longitudinal basis-perhaps 24 months. This could be followed by a recruitment process based on consistently observed savings (or lack thereof) over the longitudinal period to conduct structured debriefings of households showing various effects. 
A third methodological issue concerns the sample size and variability of the studies. In general, the samples were limited to several hundred households, which were as homogeneous as possible within the research design. It is an open question as to what the conservation and efficiency potential is for a much wider range of domestic arrangements. Household composition has been shown to be a significant predictor of energy use, but much less is known about conservation and efficiency potential. It has been shown that larger effects are obtained for high versus low consumers, with some potential for rebound at the low end of usage. Large-scale smart grid/metering implementation plans will cover a much greater range of domestic arrangements, and it will be important to evaluate conservation and efficiency effects in terms of a domestic segmentation analysis.

A final methodological issue relates to the behavioral granularity of the conservation and efficiency effects reported. While it is known that savings occur, how the savings were actually achieved is not clear because structured debriefings or household task analyses were not a routine part of many of the studies. There is considerable room in new research to address the question of: "what is happening behaviorally behind the meter?" A variety of surveys provides suggestions for implementing and gathering information on those actions that are easiest for consumers to execute and would yield consistent savings (Woods 2008; Secrest 2005). Addressing behavioral specificity would be a natural part of any large-scale implementation and would be a fundamental part of evaluating long-term savings, or lack thereof.

A number of additional issues pertinent to consumer behavior are worthy of consideration in future field research. They might be part of a large-scale study, follow-up analyses to work already performed, or potentially surveys that are aimed at better understanding likely savings effects and behavioral "entry points" for various groups of consumers. Among the issues to be addressed are:

- The role of habit, behavior change, and cognitive control. Cognitive psychology offers concepts and theories suggesting that conservation behavior is a new habit requiring skill learning. Incorporating approaches from cognitive behavior modification and human factors engineering into smart meter implementations could entail such manipulations as a pay-as-you-go system, which has been shown to be successful in Northern Ireland (Darby 2006). The meter commands attention, and ultimately financial resources, and would assist the end user in developing alternative energy-use habits.

- Conduct research into consumer mental models of energy-how do people think about energy? Do they think about it (other than gasoline?). Stern and Aronson (1984) proposed that over a 100-year period, people have become "energy unaware" to the point that much household usage is "invisible." System transparency is a fundamental concept in human factors engineering for complex systems, and may have some application in this area.

- In field research on interventions, it is possible to increase the time granularity of data to better understand energy usage patterns across the 24-hour period, weekly and weekend differences, and variations within time periods of the seasons. More granular data can help to identify recurring behaviors that are targets for modification.

- Develop more specific guidelines for information and outreach programs that are directly linked to social psychological findings, but are abstracted out of the numerous review papers and presented in a format that makes "best practices" more accessible to energy utilities that may want to implement a program. 
- Develop design guidelines for feedback systems (bills, meters, etc.) directly linked to social/cognitive/human factors findings (presented above)-a compendium of "best practices."

- Conduct research to better define the basis for behavior change in energy consuming activities-what is the reward system? What is the role of negative reinforcement? Can the concept of household or personal carbon allowances find a realistic implementation?

- Conduct preference and rating studies using survey methods to understand consumer perceptions of risks and benefits associated with various change scenarios (this can be used to quickly evaluate potential tradeoffs, such as time-based shut down of "always on" devices versus centrally-driven price increases for power usage at unnecessary times-e.g., all those home computers trickle-charging all night long.

- How can social or behavioral constraints be built into the energy consumption system that will "nudge" people to make desirable energy choices? There are a many possibilities here, some of which may be device-specific human factors design questions such as grid-friendly appliances, and others that may be longer-term life choice and land-use planning questions (where to live).

- Conduct longitudinal assessments of persistence across different types of feedback, including automation that would enable consumers to pre-set price response behavior (with an opt-out feature) that would enable in-home uses respond to price signals from the utility. Such an assessment would track the various dimensions of persistence, such as the physical, behavioral, and economic.

To summarize, there is clearly a potential for reducing GHG emissions through end-use energy conservation and efficiency by means of providing smart grid/meter feedback to households. Although a variety of questions remain, as described in this section, the time seems right to take the next step in smart grid/metering demonstration programs by extending them to wider geographic regions and much larger sample sizes. Given the implementation plans that various utilities have for deploying these technologies, detailed plans for behavioral studies need to be started now.

\section{A.5 References}

Abrahamse W, L Steg, C Vlek, and T Rothengatter. 2005. "A Review of Intervention Studies Aimed at Household Energy Conservation.” Journal of Environmental Psychology 25(3):273-291.

Abrahamse W, L Steg, C Vlek, and T Rothengatter. 2007. "The Effect of Tailored Information, Goal Setting, and Tailored Feedback on Household Energy Use, Energy-Related Behaviors, and Behavioral Antecedents." Journal of Environmental Psychology 27:265-276.

Becker LJ. 1978. "Joint Effect of Feedback and Goal Setting on Performance: A Field Study of Residential Energy Conservation.” Journal of Applied Psychology 63(4):428-433.

Benders RMJ, R Kok, HC Moll, G Wiersma, and KJ Noorman. 2006. "New Approaches for Household Energy Conservation - In Search of Personal Household Energy Budgets and Energy Reduction Options.” Energy Policy 34(18):3612-3622.

Brandon G and A Lewis. 1999. "Reducing Household Energy Consumption: A Qualitative and Quantitative Field Study.” Journal of Environmental Psychology 19:75-85. 
Chassin DP and L Kiesling. 2008. "Decentralized Coordination through Digital Technology, Dynamic Pricing and Customer-Driven Control: The GridWise Testbed Demonstration Project." The Electricity Journal 21(8):51-59.

Darby S. 2006. The Effectiveness of Feedback on Energy Consumption: A Review for DEFRA of the Literature on Metering, Billing and Direct Displays. Environmental Change Institute, University of Oxford, Oxford, United Kingdom.

Ehrhardt-Martinez K. 2008. Behavior, Energy, and Climate Change: Policy Directions, Program Innovations, and Research Paths. Council for an Energy-Efficient Economy, Washington, D.C.

Elliott DB, DM Anderson, DB Belzer, KA Cort, JA Dirks, and DJ Hostick. 2004. Baseline Inputs for BEAMS. PNNL-14696, Pacific Northwest National Laboratory, Richland, Washington. Accessed December 29, 2009, at http://www.pnl.gov/main/publications/external/technical_reports/PNNL14696.pdf.

Faruqui A, S Sergici, and A Sharif. (2009). "The Impact of Informational Feedback on Energy Consumption - A Survey of the Experimental Evidence." Energy (in press). Abstract (Working Paper Series) available online at http://papers.ssrn.com/sol3/papers.cfm?abstract_id=1407701.

Firth S, K Lomas, A Wright, and R Wall. 2008. "Identifying Trends in the Use of Domestic Appliances From Household Electricity Consumption Measurements.” Energy and Buildings 40(5):926-936.

Fischer C. 2008. "Feedback on Household Electricity Consumption: A Tool for Saving Energy?" Energy Efficiency 1(1):79-104.

Gardner GT and PC Stern. 2008. "The Short List: The Most Effective Actions U.S. Households Can Take to Curb Climate Change." Environment Magazine 50(5):12-21. Accessed December 23, 2009, at http://www.sustainablenapacounty.org/site/lifestyle/home?article=55.

Geller ES. 1981. “Evaluating Energy Conservation Programs: Is Verbal Report Enough?” Journal of Consumer Research 8(3):331-335.

Hutton RB, GA Mauser, P Filiatrault, and OT Ahtola. 1986. "Effects of Cost-Related Feedback on Consumer Knowledge and Consumption Behavior: A Field Experimental Approach.” Journal of Consumer Research 13(3):327-336.

Luyben PD. 1982. "Prompting Thermostat Setting Behavior: Public Response to a Presidential Appeal for Conservation." Environment and Behavior 14(1):113-128.

McClelland L and SW Cook. 1979. Energy Conservation Effects of Continuous In-Home Feedback in All-Electric Homes.” Journal of Environmental Systems 9(2):169-173.

McMakin AH, EL Malone, and RE Lundgren. 2002. "Motivating Residents to Conserve Energy Without Financial Incentives." Environment and Behavior 34(6):848-863. 
Midden CJH, JE Meter, MH Weenig, and HJA Zieverink. 1983. "Using Feedback, Reinforcement and Information to Reduce Energy Consumption in Households: A Field-Experiment." Journal of Economic Psychology 3(1):65-86.

Sanquist TF. 2008. "Human Factors and Energy Use." Bulletin of the Human Factors and Ergonomics Society 51(11):1-3.

Secrest T. 2005. Building Technologies Residential Survey Report. PNNL-SA-70119. Pacific Northwest National Laboratory, Richland, Washington.

Sexton RJ, NB Johnson, and A Konakayama. 1987. "Consumer Response to Continuous-Display Electricity-Use Monitors in a Time-of-Use Pricing Experiment." Journal of Consumer Research 14(1):55-62.

Shui B and H Dowlatabi. 2005. "Consumer Lifestyle Approach to U.S. Energy Use and the Related $\mathrm{CO}_{2}$ Emissions.” Energy Policy 33:197-208.

Staats H, P Harland, and HAM Wilke. 2004. "Effecting Durable Change. A Team Approach to Improve Environmental Behavior in the Household." Environment and Behavior 36(3):341-367.

Stern PC and A Aronson, eds. 1984. Energy Use: The Human Dimension. W.H. Freeman and Company, New York.

Ueno T, F Sano, O Saeki, and K Tsuji. 2006. "Effectiveness of an Energy-Consumption Information System on Energy Savings in Residential Houses Based on Monitored Data." Applied Energy 83(2):166-183.

Winett RA, MS Neale, and HC Grier. 1979. Effects of Self-Monitoring and Feedback on Residential Electricity Consumption." Journal of Applied Behavior Analysis 12(2):173-184.

Winett RA, SQ Love, and C Kidd. 1982. "The Effectiveness of an Energy Specialist and Extension Agents in Promoting Summer Energy Conservation by Home Visits." Journal of Environmental Systems 12(1):61-70.

Winett RA, IN Leckliter, DEChinn, B Stahl, and SQ Love. 1985. "Effects of Television Modeling on Residential Energy Conservation." Journal of Applied Behavior Analysis 18(1):33-44.

Wood G and M Newborough. 2007. "Energy-Use Information Transfer for Intelligent Homes: Enabling Energy Conservation with Central and Local Displays." Energy and Buildings 39(4):495-503.

Woods J. 2008. "What People Do When They Say They are Conserving Electricity." Energy Policy 36(6):1945-1956. 


\section{Mechanism B: Joint Marketing of Energy Efficiency and Demand Response Programs}

\section{B.1 Introduction}

This topic examines the synergy that may exist in the cost of administering energy efficiency and demand response programs together, rather than separately, to address the curtailment and efficiency behaviors of residential consumers that result in energy savings.

Curtailment involves overt repeated behaviors, such as turning off lights or keeping thermostats at a lower level, while efficiency behaviors involve one-time actions such as insulating or changing light bulbs to compact fluorescent light bulbs (Gardner and Stern 2008). Consumers generally associate energy savings with curtailment actions, although analysis indicates that efficiency actions can yield the largest potential savings.

Utilities and other organizations administer demand-side management programs to influence these behaviors, generally through incentive programs and price signals in the case of curtailment, and information programs in the case of efficiency. The program offerings are often administered separately, which provides the opportunity to combine the program offerings to reduce the administration cost and/or increase the savings from curtailment and efficiency programs, which is the topic of this paper.

\section{B.2 Review}

Demand response is most closely associated with curtailment behavior, as distinct from energy efficiency measures and behavior. ${ }^{1}$ While often achieving similar goals, Table B.1 outlines how energy efficiency and demand response can be differentiated based on the primary focus of their application and the expected result of implementation. As noted in Table B.1, demand response is principally designed to reduce peak/critical loads while energy efficiency is designed to reduce overall energy consumption. Thus, demand response concepts may result in load shifting and, in turn, may or may not provide realizable energy savings. Energy efficiency is designed to generate long-lived savings while demand response is designed to respond in a shorter time period to specific surges in peak power load (Nemtzow 2006). Demand response, it should be noted, can also result in energy efficiency gains, as documented later in this topic.

\footnotetext{
${ }^{1}$ Among the benefits provided by the smart grid technology is the ability to automate demand response, whereby the customer agrees to allow the utility to remotely control certain loads during certain times and/or price events.
} 
Table B.1. Key Distinctions Between Energy Efficiency and Demand Response

\begin{tabular}{ccc}
\hline & Energy Efficiency & Demand Response \\
\hline Primary Focus & Overall Energy Consumption & Peak Power load \\
Advance Notice & $\begin{array}{c}\text { Permanent and usually “always } \\
\text { on" }\end{array}$ & $\begin{array}{c}\text { Dispatchable at peak load (or as } \\
\text { needed) for hours at a time }\end{array}$ \\
Key Societal Benefits & $\begin{array}{c}\text { Months to indefinite } \\
\text { Avoid supply-side costs; avoid } \\
\text { environmental impacts; and lower } \\
\text { customer costs }\end{array}$ & $\begin{array}{c}\text { Avoid on-peak-load supply-side } \\
\text { costs; reliability; and lower price } \\
\text { volatility }\end{array}$ \\
Measured in Avoided Units & MWh, money, and power plants & $\begin{array}{c}\text { MW, money, and reliability } \\
\text { concerns }\end{array}$ \\
\hline
\end{tabular}

Source: Nemtzow (2006).

In 2008, the Federal Energy Regulatory Commission (FERC) estimated that $8 \%$ of U.S. customers were enrolled in some form of demand response program and that the contribution of all demand response programs totaled $41,000 \mathrm{MW}$ or $5.8 \%$ of U.S. peak load demand. This level of penetration represented a 9\% increase over 2006 levels. Demand response programs, as defined by FERC, included incentivebased programs (e.g., direct load control, demand bidding/buyback programs, interruptible/curtailable rates) and time-based rates (e.g., time-of-use, critical-peak-load pricing, real-time pricing) (FERC 2008).

To the extent that peak load demand reduction is the target, energy efficiency and demand response programs are complementary. In 2003, energy efficiency and demand response programs were estimated to have made peak load demand reductions of 13,581 MW and 4,000 MW, respectively (Nadel et al. 2005). The contribution of energy efficiency and demand response has grown in recent years and is expected to expand significantly in the future.

The Electric Power Research Institute (EPRI) recently completed a study that estimates energy efficiency programs in the United States have the potential to reduce electricity consumption by 398 to $544 \mathrm{~B} \mathrm{kWh}$ in 2030 (EPRI 2009). The realistic achievable potential case estimates energy efficiency gains realized by energy efficiency programs at 20.6 B kWh in 2010, $206 \mathrm{~B} \mathrm{kWh}$ in 2020, and $398 \mathrm{~B}$ $\mathrm{kWh}$ in 2030.

The EPRI study also found that the combination of energy efficiency and demand response programs could reduce summer peak load demand by $157 \mathrm{GW}$ to $218 \mathrm{GW}$, or $14 \%$ to $20 \%$ by 2030 (EPRI 2009). Table B.2 summarizes the potential reductions in summer peak load demand, as estimated in EPRI 2009, for 2010, 2020, and 2030. Estimates are presented for a realistic achievable and a maximum achievable scenario. 
Table B.2. Estimated Realistic and Maximum Potential Reductions in Summer Peak Load Demand due to Energy Efficiency and Demand Response Measures, GW

\begin{tabular}{|c|c|c|c|c|c|}
\hline \multirow[b]{2}{*}{ Year } & \multicolumn{2}{|c|}{ Energy Efficiency } & \multicolumn{2}{|c|}{ Demand Response } & \multirow[b]{2}{*}{ Total } \\
\hline & $\begin{array}{c}\text { Realistic } \\
\text { Achievable } \\
\text { Potential }\end{array}$ & $\begin{array}{c}\text { Maximum } \\
\text { Achievable } \\
\text { Potential }\end{array}$ & $\begin{array}{c}\text { Realistic } \\
\text { Achievable } \\
\text { Potential }\end{array}$ & $\begin{array}{c}\text { Maximum } \\
\text { Achievable } \\
\text { Potential }\end{array}$ & \\
\hline 2010 & $\begin{array}{c}2 \mathrm{GW} \\
(0.2 \%)\end{array}$ & $\begin{array}{l}56 \mathrm{GW} \\
(5.0 \%)\end{array}$ & $\begin{array}{l}22 \mathrm{GW} \\
(2.0 \%)\end{array}$ & $\begin{array}{l}56 \mathrm{GW} \\
(5.0 \%)\end{array}$ & $\begin{array}{l}24-112 \mathrm{GW} \\
(2.2-10.0 \%)\end{array}$ \\
\hline 2020 & $\begin{array}{l}40 \mathrm{GW} \\
(3.6 \%)\end{array}$ & $\begin{array}{l}95 \mathrm{GW} \\
(8.5 \%)\end{array}$ & $\begin{array}{l}52 \mathrm{GW} \\
(4.6 \%)\end{array}$ & $\begin{array}{l}76 \mathrm{GW} \\
(6.8 \%)\end{array}$ & $\begin{array}{l}92-171 \mathrm{GW} \\
(8.2-14.3 \%)\end{array}$ \\
\hline 2030 & $\begin{array}{l}79 \mathrm{GW} \\
(7.0 \%)\end{array}$ & $\begin{array}{l}118 \mathrm{GW} \\
(10.5 \%)\end{array}$ & $\begin{array}{l}79 \mathrm{GW} \\
(7.0 \%)\end{array}$ & $\begin{array}{c}100 \mathrm{GW} \\
(8.9 \%)\end{array}$ & $\begin{array}{c}157-218 \mathrm{GW} \\
(14-20 \%)\end{array}$ \\
\hline
\end{tabular}

Source: EPRI 2009

EPRI estimates the costs to implement the energy efficiency and demand response programs generating the energy efficiency gains outlined previously at $\$ 1 \mathrm{~B}$ to $\$ 2 \mathrm{~B}$ in $2010, \$ 8$ B to $\$ 20 \mathrm{~B}$ in 2020 , and $\$ 19$ B to $\$ 47$ B in 2030 (EPRI 2009). EPRI also estimated the levelized cost for energy efficiency $(\$ / \mathrm{kWh})$ and demand response $(\$ / \mathrm{kW}$-year) measures. The levelized costs for energy efficiency measures were estimated at $\$ .0217 / \mathrm{kWh}$ in $2010, \$ .0264$ in 2020 , and $\$ .0322$ in 2030 . The levelized costs for demand response measures were estimated at $\$ 50.70, \$ 61.81$, and $\$ 75.34 / \mathrm{kW}$-year for 2010,2020 , and 2030, respectively.

EPRI compared these results to those found in other recent studies. One study conducted by the Midwest Energy Efficiency Alliance) calculated an average levelized cost for energy efficiency measures at $\$ .10 / \mathrm{kWh}$. A second study conducted by American Council for an Energy Efficient Economy focused on energy efficiency potential in Florida estimated the levelized cost of energy efficiency measures for residential consumers to be $\$ .035 / \mathrm{kWh}$. The final examination was conducted by the authors of the EPRI report on planned expenditures by California investor-owned utilities during the 2009-2011 program cycle. This analysis yielded levelized costs of $\$ .07 / \mathrm{kWh}$ (EPRI 2009).

In preparing this paper, the author made several contacts with experts in energy efficiency and demand response fields but was unable to identify program data or literature, which could be used to estimate the administrative cost reductions potential through the integration of energy efficiency and demand response programs. Further, the contacted experts knew of few utilities employing integrated approaches. This conclusion is supported by the findings of York and Kushler (2005):

"Overall, the experts we interviewed were virtually unanimous in responding that they knew of few or no examples of programs that had explicitly attempted to integrate energy efficiency and demand response objectives and measures into a single program. At the same time, most of our interviewees felt that such an integrated approach had some conceptual merit and was worth testing."

Though energy efficiency and demand response integration is limited, there are a number of possible synergies between energy efficiency and demand response programs and measures, including:

- Energy efficiency can be used to reduce demand permanently, including during peak load hours. 
- Programs that focus on reducing peak load demand can identify inefficient and non-essential energy uses.

- Technologies that respond to demand surges also promote energy efficiency.

- Customer experience gained through demand response programs promotes more efficient use of energy.

- Customers who participate in demand response programs are more likely to participate in future energy efficiency programs once an understanding of program benefits is reached.

- Co-marketing of energy efficiency and demand response programs and measures is possible.

From the standpoint of the customer interface, there are drivers for combining energy efficiency and demand response programs. These include: a) customers are more focused on saving money as opposed to saving energy or capacity, and b) the demand response automation made possible by smart grid technology makes the curtailment activity "invisible" to the customer, thus tying the decision to cost savings and pulling it away from direct curtailment behavior.

While similar, demand response and energy efficiency programs have certain conflicts and sources of tension between them as well, including:

- The difference in focus between energy efficiency and demand response programs (one being focused on specific time/demand periods with the other focused on general energy efficiency gains) can lead to competing choices regarding technologies and plans of action.

- The marketing message may differ as energy efficiency is designed to reduce use while receiving the same service and the demand response message is tied to shifting load or getting by with less energy for short periods.

- Energy efficiency can reduce the baseline against which demand response savings are calculated, thus reducing the financial benefits of demand response programs.

- There are institution barriers to energy efficiency/demand response integration, including how to allocate costs/benefits among customers, utilities, and service providers (York and Kushler 2005).

Despite these conflicts and tensions, there are a small number of agencies and service providers that have used an integrated approach when offering energy efficiency and demand response programs and measures. One such example is the New York State Energy Research and Development Authority (NYSERDA), which offered a menu of energy efficiency and demand response options to commercial and industrial customers through its Peak Load Reduction Program (PLRP). The PLRP was originally designed to reduce summertime peak load, but was modified to include permanent demand reduction through energy efficiency. The PLRP has four main program components:

- permanent demand reduction efforts (PDRE)

- load curtailment/shifting (LC/S)

- dispatchable emergency generator initiatives (DEGI)

- interval meters (IM). 
The program includes a reimbursement incentive, aggregation incentive, and a controllable appliance aggregation incentive. PLRP incentives differ by program component with PDRE, receiving the largest incentive at $\$ 225-\$ 475 / \mathrm{kW}$, while LC/S incentives are $\$ 45-\$ 175 / \mathrm{kW}$ and DEGI incentives are $\$ 125 / \mathrm{kW}$. Interval meter incentives are $\$ 1200$ to $\$ 2500 /$ meter.

The evaluation results from 2001 to 2003 are presented in Table B.3. The program resulted in a $355,302 \mathrm{~kW}$ reduction in demand, with $49 \%$ of the savings realized through IM and $27 \%$ realized through LC/S. PDRE generated the level of savings among the four programs, accounting for $4 \%$ of total savings. The realization rates shown in Table B.3 represent the ratio of M\&V adjusted program savings to initial estimates of project savings (York and Kushler 2005).

Table B.3. NYSERDA PLRP Demand Reductions and Realization Rate, 2001-2003

\begin{tabular}{ccc}
\hline Program Path & $\begin{array}{c}\text { M\&V Evaluation-Adjusted } \\
\text { Demand Reduction }(\mathrm{kW})\end{array}$ & $\begin{array}{c}\text { Realization Rate } \\
\text { (actual as \% of estimated) }\end{array}$ \\
\hline PDRE & 14,993 & 102 \\
LC/S & 95,912 & 104 \\
DEGI & 69,729 & 100 \\
IM & 174,668 & 88 \\
\hline PLRP Total & 355,302 & \\
\hline Source: York and Kushler (2005) & & \\
\hline
\end{tabular}

Integrated energy efficiency/demand response approaches have also targeted residential customers. The Chicago-based Community Energy Cooperatives launched a residential integrated energy efficiency/demand response program in 2002 called the Energy-Smart Pricing Plan (ESPP). The program was designed to provide customers with tools and information to better manage their energy use based on price signals given by the market. In the ESPP, customers received day-ahead price information and special alerts regarding price spikes. In the first year of the program (2004), customers saved approximately $\$ 12 /$ month (equivalent to a $20 \%$ reduction in price). Program activity suggests the program was immediately effective. Of those customers participating in the ESPP, $76 \%$ indicated that they now turn off lights more, $28 \%$ were more likely to turn up their air conditioner settings, $50 \%$ were more likely to do laundry at night, and 13\% installed insulation or weather stripping (York and Kushler 2005).

There is also a technological overlap between energy efficiency and demand response. For example, energy management and control systems (EMCS) enable customers to monitor, analyze, and control building systems and equipment to run in an energy efficient manner. EMCS technology can both reduce peak load energy demand and promote energy efficiency. EMCS applications continue to expand in the United States. In 2003, 7\% of commercial buildings covering $31 \%$ of total square footage nationwide used an EMCS. Energy savings due to EMCS deployment is estimated at $10 \%$ to $20 \%$ of total energy demand (Kiliccote and Piette 2005). 
Lighting controls also characterize the technology overlap between energy efficiency and demand response. While light controls have exclusively demand response (e.g., demand limiting, overrides) and energy efficiency (e.g., on/off controls, clocks/timers, manual dimming) components, a small number of capabilities address both energy efficiency and demand response objectives (e.g., central dimming, bilevel/zonal switching). HVAC control technologies hold similar overlapping capabilities, including global zone setup/feedback and duct static pressure reduction (Kiliccote and Piette 2005).

Due to these efficiency benefits, energy efficiency and demand response programs and measures hold great potential for reducing the environmental impacts of energy generation systems on the environment. Energy efficiency and demand response programs result in the conservation effect already touched on in this paper but also benefit the environment through load flattening, which defers or eliminates the need for additional power lines and power plants. Further, demand response alters the generation mix by fuel and by unit and also fits in well with intermittent renewables (Nemtzow 2006).

The environmental benefits of integrated energy efficiency/demand response approaches could include reductions in emissions. Figure B.1 presents a modeled estimate of the annual air impacts demand response with energy-targeted efficiency (always-on) and energy efficiency targeting peak load periods. The two types of energy efficiency approaches provide for a greater absolute reduction in emissions primarily because they are always on (Synergy Energy Economics 2003).

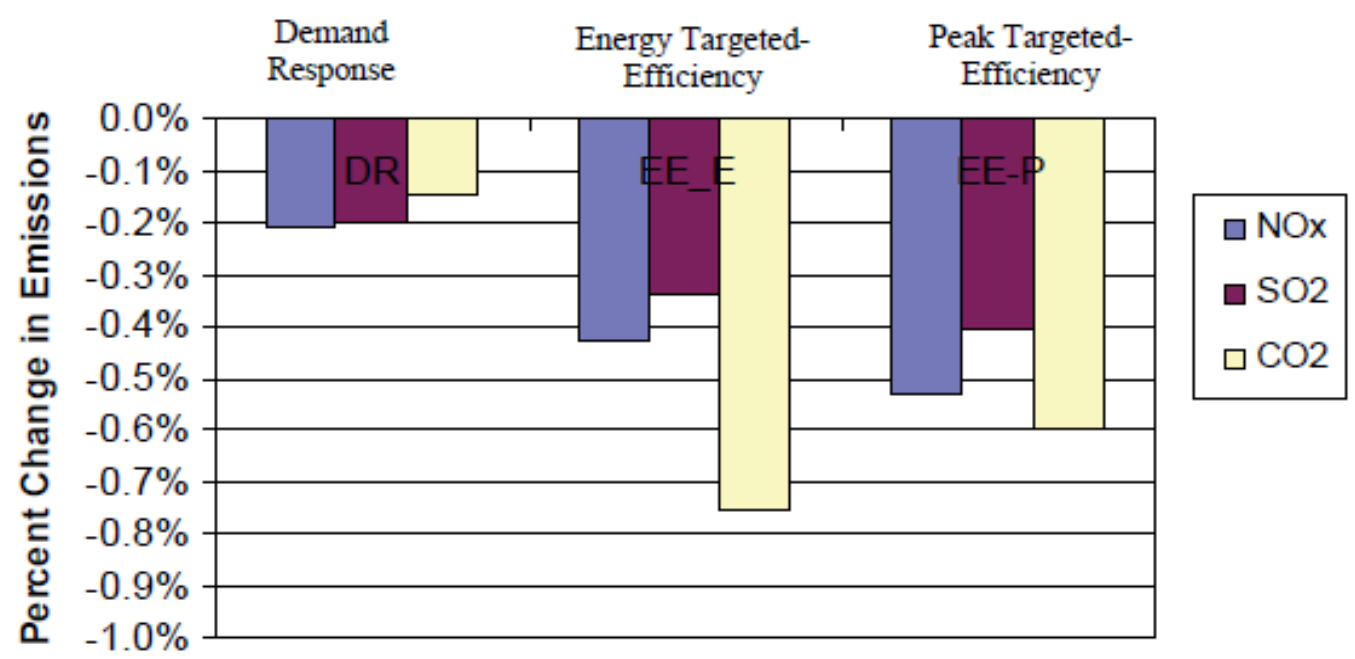

Figure B.1. Comparison of Annual Emissions for Demand Response, Energy-Targeted Efficiency, and Peak-Load-Targeted Efficiency Programs. (Source: Synapse Energy Economics, Inc.)

The question remains, however, how can a utility best integrate energy efficiency and demand response programs and measures to reduce administrative costs? Gardner and Stern (2008) provide a short list of effective actions that residential energy consumers can take based on potential savings. Some of the largest savings would result from efficiency actions such as properly insulating older homes. However, there are a variety of complicating factors that impede taking these actions, including the expenditures required, the lack of understanding regarding payback period, and the general complexity of finding and evaluating contractors and overseeing the work. 
Basic human factor engineering principles suggest that integrating and tailoring energy savings information is a desirable goal. By reducing the number of channels of data, consumer attention can be focused (Sanquist 2008). Similarly, if the smart meter in the household is viewed as an energy saving tool, the effect is likely to extend to the smart grid/meter as an information source for further savings. This latter effect will depend largely on the extent to which the diverse information regarding energy efficiency behaviors can be presented in a meaningful way in the home. A starting point might be to provide ranked lists of potential actions (such as the Gardner and Stern's short list) tailored to data regarding usage patterns available via smart metering.

\section{B.3 Summary}

Combining the administration of energy efficiency and demand response programs is expected to result in cost savings that could be reinvested in energy efficiency programs and/or increase program effectiveness. However, the literature review and contact with experts in the energy efficiency and demand response fields did not reveal any program data or information that could be used to estimate administrative cost reductions or increases in program effectiveness. Based on this, indirect reductions in electricity and $\mathrm{CO}_{2}$ emissions calculated in Attachment 2 are estimated to be zero, as there is not a basis

for determining administrative cost reductions or increases in program effectiveness. No direct reductions in electricity or capacity are expected.

\section{B.4 Recommendations for Additional Work and/or Consideration}

- An analysis of the impact of co-administering energy efficiency and demand response programs. That is, if energy efficiency and demand response programs are administered together, by what percent could the total technology/administrative overhead costs be reduced? A quote from Jesse Berst (2009) indicates thought on this recommendation-"Rising costs? We'll just have the electric power industry integrate energy efficiency and demand response."

- An analysis of how utilities organizationally typically deliver energy efficiency and demand response programs (together or separate).

- Examine how the implementation of smart grid technologies influences the effectiveness (e.g., energy, capacity, and utility cost) of merging energy efficiency and demand response administrative / delivery structures.

\section{B.5 References}

Berst J. 2009. "Four Keys to Managing Smart Grid Evolution." SmartGridNews.com. Accessed December 29, 2009, at http://www.smartgridnews.com/artman/publish/commentary/Four_Keys_to_Managing_Smart_Grid Evolution-564.html.

Electric Power Research Institute (EPRI). 2009. Assessment of Achievable Potential from Energy Efficiency and Demand Response Programs in the U.S.: (2010-2030). 1016987, EPRI, Palo Alto, California. Accessed December 20, 2009, at http://mydocs.epri.com/docs/public/000000000001018363.pdf. 
Federal Energy Regulatory Commission (FERC). 2008. Assessment of Demand Response and Advanced Metering: Staff Report. Federal Energy Regulatory Commission, Washington, D.C.

Gardner GT and PC Stern. 2008. "The Short List: The Most Effective Actions U.S. Households Can Take to Curb Climate Change.” Environment Magazine 50(5):12- 21.

Keith G, B Biewald, D White, and M Drunsic. 2003. Modeling Demand Response and Air Emissions in New England. Prepared by Synapse Energy Economics, Inc. for the U.S. Environmental Protection Agency. Cambridge, Massachusetts.

Kiliccote S and M Piette. 2005. "Advanced Control Technologies and Strategies Linking Demand Response and Energy Efficiency." LBNL-58179. Proceedings of the Fifth Annual International Conference on Enhanced Building Operations. Texas A\&M University, College Station, Texas.

Nadel S, D York, and M Kushler. 2005. "Introduction and Overview: Demand Response and Energy Efficiency Today and Tomorrow: What are the Opportunities?" Presented at the American Council for an Energy-Efficient Economy, National Association of Regulatory Commissioners, Washington, D.C. Accessed December 29, 2009, at http://www.narucmeetings.org/Presentations/nadelyorkkushler_w05.pdf.

Nemtzow D. 2006. "Demand Response, Energy Efficiency and the Environment: What's the Relationship." U.S. Demand Response Coordination Committee, Washington, D.C.

Sanquist TF. 2008. "Human Factors and Energy Use." Bulletin of the Human Factors and Ergonomics Society 51(11):1-3.

York D and M Kushler. 2005. Exploring the Relationship between Demand Response and Energy Efficiency: A Review of Experience and Discussion of Key Issues. U052, American Council for an Energy-Efficient Economy, Washington, D.C. Accessed December 29, 2009, at http://www.aceee.org/pubs/u052.pdf. 


\section{Mechanism C: Deployment of Diagnostics in Residential and Small/Medium Commercial Buildings}

\section{C.1 Introduction}

This topic examines the application of smart-grid-enabled diagnostics to heating, ventilating, and air conditioning (HVAC) systems in residential and small/medium commercial buildings. This is feasible because the HVAC systems are reasonably similar in design and function, and the smart grid's real-time sensing and communication technology enables automated profiling of these systems to detect malfunctions at an early stage. The application of diagnostics to HVAC systems in large commercial buildings is becoming routine practice and can be administered through building automation systems; this reduces the need for smart-grid-enabled diagnostic tools, at least in the foreseeable future.

For more than a decade, researchers have heralded the imminent rise of home automation and smart appliances as a way to increase home comfort and functionality while reducing energy consumption and emissions. Although this has yet to become a reality, several trends appear to make this vision more feasible today, including:

- widespread internet connectivity, including broadband deployment

- continuing decreases in the cost of computing power, communications, power electronics, and sensors

- greater homeowner acceptance and comfort with home electronics

- rapidly growing use of microprocessors and programmable controls in home appliances and HVAC equipment

- widespread adoption of automated meters with two-way communication capabilities.

In addition to the above trends, the smart grid provides access to information that was not previously available: whole-house or building electricity consumption, thermostat status, run-times and duty cycles of major equipment, current and future weather information, calendar information (time of day/year), and current and future price of electricity. All of this information can be used not only to optimize the energy use and reduce the operating cost, but also as potential help in increasing the operating efficiency of major appliance and energy-consuming equipment in homes and buildings.

\section{C.2 Review}

HVAC system operation degrades due to inoperable dampers, dirty/clogged filters and coils, incorrect refrigerant charges, failing compressors, failed sensors, failed fans, missing enclosure panels, incorrectly implemented controls, excessive run-time, and other conditions. These lead to increased energy consumption, higher operating cost, shortened equipment life, and unhealthy conditions for building occupants. Customers are often not aware of these conditions, nor do they have the motivation or expertise to detect and resolve many of the conditions. Resolution typically requires a service call to have a trained individual conduct an inspection, often after a condition has progressed to a detectable level (insufficient heating/cooling capacity, which leads to discomfort). 
The smart grid provides automated real-time sensing and communication. This, coupled with a centralized diagnostician, enables the operating signature to be developed for individual pieces of HVAC equipment, which in turn enables automated fault detection and diagnostics (AFDD) technologies to diagnose deviations from this profile. Detection and diagnosis can be conducted automatically and comprehensively without the ongoing cost of expensive human expertise by embedding the expertise required to detect and diagnose operational problems in software tools. Furthermore, when used in commissioning, these tools can remain as a legacy in buildings after they are constructed, to protect building systems against slow mechanical degradation and/or faults inadvertently introduced by operators seeking to resolve complaints without finding root causes.

\section{Smart HVAC Systems}

Smart systems can automate activities to include simple housekeeping measures, such as setting back thermostats (which can reduce heating energy consumption by $\sim 10 \%$ by turning the thermostat back $10 \%$ to $15 \%$ for 8 hours (DOE/EERE 2009). Cooling setup can yield similar or greater savings per degree of setup) and scheduling routine maintenance of HVAC systems. Often, however, occupants do not carry out these basic measures, e.g., thermostat night setback rates of greater than $5^{\circ} \mathrm{F}$ appear to range between 20\% (manual) and 35\% (programmable) (Nevius and Pigg 2000). In fact, one study suggests that programmable thermostats may achieve negligible energy savings relative to manual thermostats due to human factors. "Smart" energy systems can "learn" how occupants use energy and provide control options that reduce energy use, energy waste, and energy cost.

The customer can be notified that the equipment performance is degraded and informed of recommended actions that may be taken, the likely cost to resolve the condition, and the savings that would be realized. The customer can, on her/his own volition, make the choice to correct the condition or defer that action to the utility (potentially as an integral part of energy efficiency and demand response programs). The benefits to the customer are lower energy bills, longer equipment life, and a healthier environment. Social benefits are reduced $\mathrm{CO}_{2}$ emissions and, possibly, increased energy security in cases that the energy savings are from imported fossil fuels.

In addition to energy and emission reductions, many other benefits can be directly attributed to this technology including: 1) better peak load management, which allows for utility distribution and transmission and distributions companies to defer updates, and 2) better management of onsite generation. McKinsey \& Company ${ }^{1}$ estimates a cost savings to the customer of between $\$ 5 \mathrm{~B}$ to $\$ 8$ B dollars annually if 5\% to $8 \%$ of the residential peak load is shifted (from all appliances) to off-peak load and curtail an additional $4 \%$ to $7 \%$ of resulting peak load. The savings are estimated using the actual timevarying prices for the PJM (Delaware, Illinois, Indiana, Kentucky, Maryland, Michigan, New Jersey, North Carolina, Ohio, Pennsylvania, Tennessee, Virginia, West Virginia, and the District of Columbia) power pool. Other studies have reported possible peak load reductions of $10 \%$ to $15 \% .^{2}$ In addition, improved maintenance would also reduce emissions of chlorofluorocarbon (CFC) refrigerants for those units not yet using hydro-chlorofluorocarbon (HCFC) refrigerants. Furthermore, better maintenance leads to longer equipment lives, reduced waste, and extended landfill lives.

In the case of small/medium commercial buildings, rooftop packaged cooling and packaged heat pump equipment is most amenable to AFDD. Packaged cooling equipment is used in $42 \%$ of all commercial buildings $(19.7 \mathrm{M})$, serving over $54 \%$ of the commercial building floor space in the United States (EIA 2002) (36.5 $\mathrm{B} \mathrm{ft}{ }^{2}$ ). The primary cooling energy consumption of rooftop packaged and unitary

\footnotetext{
${ }^{1}$ http://energycommerce.house.gov/107/hearings/06222001Hearing265/swofford.pdf

${ }^{2}$ http://gridwise.pnl.gov/docs/pnnl14396.pdf and http://www.rand.org/pubs/technical_reports/TR160/index.html.
} 
cooling equipment is about 1.03 quads annually, and packaged heat pumps use an additional 0.1 quad of source energy. As with residential, there are no reliable estimates for equipment operation, but it is estimated that savings of $10 \%$ to $30 \%$ of the energy consumed by these units is achievable by correcting operation problems (Ardehali and Smith 2002; Claridge et al. 1996; EIA 2002). Conservatively, 10\% to $20 \%$ is attributed to soft failures that go unnoticed and are compensated by equipment overwork, and the additional $10 \%$ is due to hard failures that require a service call. In the case of residential systems, there are no reliable estimates available on the operation of HVAC appliances nationwide, but many regional studies have noted that HVAC systems are not properly maintained in many homes, leading to $15 \%$ to $30 \%$ in HVAC energy waste (Neme et al. 1999).

\section{C.3 Summary}

Buildings connected to the smart grid can save energy and money through enhanced operation from actions as simple as night setback to responding to time-of-use and/or peak load energy prices. A second service that the smart grid can provide is scheduling routine maintenance. Improved maintenance would also reduce emissions of CFC refrigerants for those units not yet using HCFC refrigerants. Furthermore, better maintenance leads to longer equipment lives, reduced waste, and extended landfill lives. The third service is AFDD to remotely identify and diagnose real and potential problems. There is considerable value to customers in detecting and repairing heating and air-conditioning equipment before it fails to provide comfort and requires service. The reductions in electricity and $\mathrm{CO}_{2}$ emissions calculated in Attachment 2 are based on the literature review and the author's experience. It is estimated that direct reductions are $15 \%$ in residential sectors for heating and cooling electricity, with a range of $10 \%$ to $20 \%$, and $20 \%$ in small/medium commercial building sectors for HVAC and lighting, and a range of $10 \%$ to $30 \%$, can be achieved through implementation of smart grid technologies. No indirect reductions in electricity or capacity are expected.

\section{C.4 Recommendations}

Many technologies are required to enable diagnostics in buildings through the smart grid. Some of these are being developed and marketed today; others are missing from the marketplace. Generic technologies that are needed or could contribute to cost-effective automated energy management include:

- technologies that enhance system operation in residential and small commercial buildings

- automated fault detection and diagnostics

- automated commissioning

- condition-based maintenance

- ventilation system management

- technologies that facilitate peak load management

- smart thermostat with local/remote communication capabilities for residential and small commercial buildings

- price-based controls for large commercial buildings that have building automation systems

- technologies that allow for coordination and integration with other systems in residential and small commercial buildings. 


\section{C.5 References}

Ardehali MM and TF Smith. 2002. Literature Review to Identify Existing Case Studies of ControlsRelated Energy-Inefficiencies in Buildings. ME-TFS-01-007. Department of Mechanical and Industrial Engineering, University of Iowa, Iowa City, Iowa.

Claridge DE, M Liu, Y Zhu, M Abbas, A Athar, and JS Haberl. 1996. "Implementation of Continuous Commissioning in the Texas LoanSTAR Program: Can You Achieve 150\% Estimated Retrofit Savings Revisited?" In Proceedings of the 1996 ACEEE Summer Study on Energy Efficiency in Buildings. American Council for an Energy Efficient Economy, Washington, D.C.

DOE/EERE - U.S. Department of Energy/Office of Energy Efficiency \& Renewable Energy. 2009. "Programmable Thermostats." In: Energy Efficiency \& Renewable Energy. U.S. Department of Energy, Washington, D.C. Accessed December 29, 2009, at: http://www1.eere.energy.gov/consumer/tips/thermostats.html.

EIA - U.S. Energy Information Administration. 2002. 1999 Commercial Building Energy Consumption and Expenditures (CBECS) Tables. U.S. Energy Information Administration, Washington, D.C. Accessed December 29, 2009, at http://www.eia.doe.gov/emeu/cbecs/detailed tables 1999.html.

Neme C, J Proctor, and S Nadel. 1999. National Energy Savings Potential From Addressing Residential HVAC Installation Problems. American Council for an Energy Efficiency Economy, Washington, D.C.

Nevius M and S Pigg. 2000. "Programmable Thermostats that Go Berserk? Taking a Social Perspective on Space Heating in Wisconsin." Proceedings of the American Council for an Energy Efficient Economy (ACEEE) Summer Study on Energy Efficiency in Buildings. ACEEE, Washington, D.C. 


\section{Mechanism D: Measurement \& Verification for Energy Efficiency Programs}

\section{D.1 Introduction}

Utilities must be able to measure and verify the savings efficacy of their programs and projects in order to gain credits for their energy efficiency efforts and to prove the prudency of their programs to state regulators. The smart grid's demand response controls/sensors and communication networks can be leveraged to measure end-use efficiency savings for every customer with greater certainty at lower cost, thereby making energy efficiency programs more valuable to utilities. This paper examines the role of measurement $\&$ verification $(\mathrm{M} \& \mathrm{~V})$ for energy efficiency programs, the benefits derived through $\mathrm{M} \& \mathrm{~V}$ technology (including cost savings), and the costs of deploying $\mathrm{M} \& \mathrm{~V}$ technology.

The purpose of $\mathrm{M} \& \mathrm{~V}$ is to improve the certainty of quantifying the energy and/or cost savings, allocating risk, and supporting any guaranteed savings. M\&V technology results in numerous benefits to projects, including:

- accurate determination of energy savings for a project

- risk allocation

- reduction in uncertainties

- persistence of savings

- monitoring of equipment performance

- detection of areas where additional savings are feasible

- enhancement of operations and maintenance

- verification that cost guarantees are met

- allowance for energy efficiency program adjustments and further monitoring (DOE/FEMP 2008).

\section{D.2 Review}

In 2005, M\&V of end-use energy efficiency costs were estimated at between $3 \%$ and $15 \%$ of total utility capital costs by DOE/FEMP (2005). Within the past few years, however, more detailed assessments of M\&V costs associated with projects undertaken through the Federal Energy Management Program's (FEMP's) Super Energy Savings Performance Contracts (ESPCs) program have resulted in more refined estimates that average $3 \%$ of total utility capital costs, with a range between $1 \%$ and $4 \%$ (DOE/FEMP 2009).

Private-sector investment associated with energy efficiency projects undertaken through ESPCs program totaled \$2.3 B from 1998 to 2007 (\$230 M annually). That level of investment was estimated to be $50 \%$ of the nation's investment in energy-efficiency-related M\&V (Shonder 2009). The 3\% estimated national investment in energy efficiency-related projects ( $\$ 460 \mathrm{M}$ annually) that require $\mathrm{M} \& \mathrm{~V}$ indicates the annual investment in $\mathrm{M} \& \mathrm{~V}$ to be approximately $\$ 13.8 \mathrm{M}$ nationally.

$\mathrm{M} \& \mathrm{~V}$ is required of utilities by state regulators to show the prudency of expending ratepayer revenues to run energy efficiency programs. It is very difficult, if not impossible, to measure savings of most energy efficiency programs by comparing before-and-after data from 12 monthly billing periods due to 
the inability of traditional meters to identify and measure energy consumption at specific times or, more directly, for specific appliances that have been installed with equipment designed to conserve energy. A modern demand response network with automated metering provides data at short intervals (hourly or less), thereby providing greater temporal resolution with which to conduct accurate $\mathrm{M} \& \mathrm{~V}$ in real time.

Further, metering equipment used in demand response programs can provide direct or proxy measurement of end-use consumption for space conditioning and water heating, the two largest residential end uses, and those with the most significant seasonal swings that confound the analysis of monthly bills. In commercial buildings, this equipment can provide direct or proxy measurement of the breakdown of HVAC refrigeration, and in some cases lighting, the primary end uses in commercial buildings. Thus, in addition to the increased temporal resolution, demand response networks can also provide highly valuable end-use resolution to improve M\&V accuracy at lower cost.

Annual M\&V costs were once estimated at 3\% to $15 \%$ of the annual savings associated with the aforementioned benefits (DOE/FEMP 2005). For example, the Texas LoanSTAR (Loans to Save Taxes and Resources) program, which is a statewide capital retrofit program for enhancing energy efficiency in buildings, had (as of November 2007) funded 191 loans valued at more than $\$ 240 \mathrm{M}$. Energy savings resulting from the projects tied to these loans had reached more than \$212 M (TSEC 2009). Through October 1999, the M\&V costs associated with the Texas LoanSTAR program were computed at $11.5 \%$ of total loan value for a five-year program. That amount was computed by adding metering costs of $3.5 \%$ of the loan and annual M\&V costs of $1.6 \%$ over five years (Turner et al. 2000). In recent years, however, more detailed assessments of $\mathrm{M} \& \mathrm{~V}$ costs have reduced and narrowed the cost range, and are currently estimated to average 3\% of the value of annual savings (Shonder 2009).

$\mathrm{M} \& \mathrm{~V}$ technologies enhance the political and regulatory landscape for utilities interested in expanding energy efficiency programs tied to the smart grid. To quantify the potential energy savings associated with enhanced M\&V capabilities, the Electric Power Research Institute (EPRI) used the findings of a study on the potential of energy efficiency measures in the United States conducted by EPRI and the Edison Electric Institute (EEI). The study, which examined stock turnover to estimate potential energy efficiency gains through 2030, used the EIA Annual Energy Outlook 2007 as the baseline forecast. The analysis yielded a $7 \%$ to $11 \%$ estimate of potential energy efficiency gains through 2030 , equivalent to 398 to $544 \mathrm{~B} \mathrm{kWh}$ of reduced energy consumption (EPRI 2009).

The EPRI/EEI study bounds its estimated energy efficiency savings based on two sets of assumptions, one deemed the realistic achievable potential $(7 \%$ reduction in baseline electricity consumption) and one identified as the maximum achievable potential (11\% reduction in baseline electricity consumption). The maximum achievable potential scenario is based on the results of the most successful energy efficiency programs in the United States while the realistic achievable scenario discounts these results based on factors that could undermine program effectiveness, such as budgetary constraints and energy efficiency learning curves.

Using the findings of the EPRI/EEI study, EPRI estimated the potential energy efficiency impact of M\&V capabilities enabled by the smart grid. First, EPRI used the range between the realistic achievable potential and maximum achievable potential $(146 \mathrm{~B} \mathrm{kWh})$ to establish the baseline against which to estimate the impact of M\&V capabilities. Second, EPRI assumed that the difference between the two scenarios (realistic achievable potential and maximum achievable potential) would be based on a number 
of factors required to produce an ideal program, such as $M \& V$ capabilities enabled by the smart grid, consumer education, externalities, regulatory regimes, and utility business models.

The 2009 Annual Energy Outlook (AEO) forecasts retail electricity prices at $10.4 \phi^{1} / \mathrm{kWh}$ in 2030 (DOE/EIA 2009). When applied to the energy savings estimated by EPRI, the total cost savings associated with the advancement of smart-grid-enabled enhanced M\&V capabilities total $\$ 1.0 \mathrm{~B}$ to $\$ 4.3 \mathrm{~B}$ in the 2030 base case.

In addition to monitoring and verifying energy savings, $M \& V$ devices also yield operational benefits for utilities through real-time observation of system performance, early detection of system problems, and analysis of system behavior. The advanced metering infrastructure (AMI), for example, has numerous capabilities that hold potential operational benefits (FERC 2007):

- remote disconnect/connect

- remote firmware upgrades

- ability to support demand response through the sending of messages to equipment at a customer's home

- notification of outage and restoration

- remote meter reading

- voltage flagging if the voltage level is not within the range that is configurable by the utility

- ability to use memory to store a number of days' readings on the meters

- prepaid metering

- capacity to include data warehousing systems

- integration of data-management systems into operations-management systems (e.g., outage management, billing, accounting)

- ability to extend smart grid and AMI to appliances located within the customer's home connected together as part of a home area network.

Full nationwide AMI deployment has been estimated at \$27 B with 50\% to $80 \%$ of the cost recovered through operational savings. Long-run generation, transmission, and distribution savings are estimated at $\$ 35 \mathrm{~B}$ while an additional $\$ 5 \mathrm{~B}$ to $\$ 10 \mathrm{~B} / \mathrm{year}$ are estimated in the form of reduced electricity prices.

Other benefits cited in the study include reduced environmental externalities, enhanced power quality and reliability, and expanded consumer choice. When all are considered, benefits exceed costs by at least a 4to-1 ratio (NETL 2008).

While the benefit potential appears significant, AMI currently comprises only approximately $4.7 \%$ of total U.S. electric meters (6.7 M meters), with states in the Mid-Atlantic, Florida, and Midwest regions (Electric Reliability Council of Texas, RFC, and Southwest Power Pool) having the highest penetration rates (approximately 5\% to 10\%) and the remaining regions with lower-than-average reported rates. The number of installed advanced meters has been projected to expand rapidly by another 52 million by 2012,

\footnotetext{
${ }^{1}$ The AEO presents price data in real terms, meaning that prices do not reflect the effects of inflation.
} 
suggesting that utilities and regulators are beginning to focus more on building a network of the future that includes AMI technology and expanded M\&V capabilities (FERC 2007).

\section{D.3 Summary}

With the advent of AMI, real-time communication systems, and advanced data-management and control strategies that make up the smart grid, $M \& V$ can provide more than verification of the energy savings achieved by energy efficiency programs. The smart grid system of technologies can also provide operational benefits for utilities through real-time observation of system performance, early detection of system problems, analysis of system behavior, and the persistence of energy efficiency measures over time.

There is no question that the smart grid system will be capable of replacing short-term $M \& V$ efforts with long-term monitoring of energy efficiency programs and measure performance, but there is currently no basis for disaggregating the costs of the smart grid system to make a comparison with traditional M\&V costs. However, a smart grid system will not be implemented only for its M\&V value. The $3 \%$ of an energy efficiency program cost typically allocated for traditional $\mathrm{M} \& \mathrm{~V}$ can be used to establish a central analysis function that will operate over the long term, rather than the traditional short-term M\&V program that expends significant resources on field measurement and one-time analyses. The savings from $\mathrm{M} \& \mathrm{~V}$ is achieved by reducing the risk of certain energy-efficient technologies, programs, and enabling utilities in combination with end users to go beyond the realistically achievable estimate of savings closer to the higher-end potential savings. Although there are no reliable estimates on how much more savings could be achieved with M\&V beyond what is realistically achievable, EPRI estimates the range to be 5\% to $20 \%$ (EPRI 2008).

The reductions in electricity and $\mathrm{CO}_{2}$ emissions calculated in Attachment 2 are based on the EPRI estimate and other information in the literature review, and the author's experience. Direct reductions achievable through implementation of smart grid technologies are estimated to be $7 \%$ in residential sectors for heating and cooling electricity, with a range of $5 \%$ to $20 \%$, and $7 \%$ in small/medium commercial building sectors for HVAC and lighting, with a range of $5 \%$ to $20 \%$. In addition, indirect reductions in electricity consumption of $1 \%$, with a range of $0 \%$ to $2 \%$, are expected for both the residential and small/medium commercial building sectors.

\section{D.4 Recommendations for Additional Work and/or Consideration}

This report summarizes the findings of recently published reports that document the benefits and costs associated with $M \& V$ equipment in a general and unsystematic manner. We recommend that the benefits and costs associated with $M \& V$ equipment be explored in a more formal cost-benefit analysis. In completing this analysis, $\mathrm{M} \& \mathrm{~V}$ benefits and costs can be explored in a systematic manner over a long time period. Such an analysis would determine the extent to which investment in M\&V is cost-beneficial.

The costs associated with M\&V equipment are estimated by DOE at 3\% to $15 \%$. Such a broad range is instructive but not very precise. This lack of precision makes it difficult for utilities to estimate the $\mathrm{M} \& \mathrm{~V}$ costs likely to be incurred when embarking on new energy efficiency programs. Thus, we recommend a more advanced process of documenting these costs in a database in a manner that will enable project sponsors to estimate potential costs and/or benefits tied to specific projects and programs. 
The proposed database could be designed to resemble the intelligent transportation systems costs (http://www.itscosts.its.dot.gov/) and benefits (http://www.itsbenefits.its.dot.gov/) database housed by the U.S. Department of Transportation.

One postulation is that the smart grid will lower M\&V costs, because the metering/sensing components and communication networks will be in place, so the $\mathrm{M} \& \mathrm{~V}$ cost will be limited to an analysis function, which could also be automated. This would enable long-term $\mathrm{M} \& \mathrm{~V}$, as well as long-term monitoring of persistence and automated diagnostics. An improved understanding of this component of the cost structure is needed to answer the question of the smart grid on the costs associated with $\mathrm{M} \& \mathrm{~V}$, persistence monitoring, and diagnostics.

\section{D.5 References}

DOE/EIA - U.S. Department of Energy/U.S. Energy Information Administration. 2009. Annual Energy Outlook. DOE/EIA-0383 (2009), U.S. Department of Energy, Washington, D.C. Accessed December 29, 2009, at: http:/www.eia.doe.gov/oiaf/aeo/.

DOE/FEMP - U.S. Department of Energy/Federal Energy Management Program. 2005. FEMP 1/2 Day $M \& V$ Training Course, Part 2: Risk and Responsibility. U.S. Department of Energy, Federal Energy Management Program, Washington, D.C. Accessed December 29, 2009, at: http://ateam.lbl.gov/mv/.

DOE/FEMP - U.S. Department of Energy/Federal Energy Management Program. 2008. $M \& V$ Guidelines: Measurement and Verification for Federal Energy Projects, Version 3.0. Prepared by Nexant for U.S. Department of Energy, Federal Energy Management Program, Boulder, Colorado. Accessed December 29, 2009, at http://ateam.lbl.gov/mv/.

DOE/FEMP - 2009. U.S. Department of Energy, Federal Energy Management Program. 2009. Financing and Pricing Evaluation Workshop for Federal ESPCs. U.S. Department of Energy, Washington, D.C.

Electric Power Research Institute (EPRI). 2008. The Green Grid: Energy Savings and Carbon Emissions Reductions Enabled by a Smart Grid. EPRI-1016905, EPRI, Palo Alto, California.

Electric Power Research Institute (EPRI). 2009. Assessment of Achievable Potential from Energy Efficiency and Demand Response Programs in the U.S.: (2010-2030). 1016987, EPRI, Palo Alto, California. Accessed December 29, 2009, at http://mydocs.epri.com/docs/public/000000000001018363.pdf.

Federal Energy Regulatory Commission (FERC). 2007. Assessment of Demand Response \& Advanced Metering: Staff Report. Federal Energy Regulatory Commission, Washington, D,C. Accessed October 13, 2008, at http://www.ferc.gov/legal/staff-reports/09-07-demand-response.pdf.

National Energy Technology Laboratory (NETL). 2008. Advanced Metering Infrastructure. Prepared by National Energy Technology Laboratory for the U.S. Department of Energy, Office of Energy Delivery and Energy Reliability, Pittsburg, Pennsylvania. Accessed October 10, 2008, at http:/www.netl.doe.gov/moderngrid/docs/AMI\%20White\%20paper\%20final\%20021108.pdf. 
Shonder J. 2009. Personnel Communication. Oak Ridge National Laboratory, Oak Ridge, Tennessee.

Texas State Energy Conservation Office (TSEC). 2009. State Energy Conservation Office. Austin, Texas. Accessed June 10, 2009, at http://www.seco.cpa.state.tx.us/ls.htm

Turner D, D Claridge, D O'Neal, J Haberl, W Heffington, D Taylor, and T Sifuentes. 2000. Program Overview: The Texas LoanSTAR Program; 1989-October 199, A 10-Year Experience. Energy Systems Laboratory, Texas A\&M University, College Station, Texas. 


\section{Mechanism E: Shifting Load to More Efficient Generation}

\section{E.1 Introduction}

Demand response and distributed storage can shift load to shoulder and/or base-load off-peak-load periods and result in reductions in primary energy consumed and $\mathrm{CO}_{2}$ emissions. Demand response is most often used to shift loads from inefficient ( $\leq 30 \%$ for simple cycle combustion turbines [CTs]) to shoulder periods to more efficient $(\sim 40 \%)$ natural-gas-fired combined-cycle power plants, and have lower carbon emissions per unit of input energy. In cases where demand response shifts loads to base-load power plants that are not coal-fired, carbon savings also ensue due to the less carbon-intensive input fuels. The current mix of U.S. base-load power is expected to get cleaner as more renewable, nuclear, and clean-coal-fired power plants enter the system, which will further increase emissions reductions. This section estimates the potential for reducing energy and $\mathrm{CO}_{2}$ emissions from load-shifting strategies.

\section{E.2 Review}

California established the "Shift \& Save" program targeted to load-shifting applications using thermal energy storage systems in 2007. The stated reduction in the carbon footprint was estimated to be between $10 \%$ and $20 \%$ for each participant enrolled in the program (Cypress 2007). The underlying mechanism for the reduction in the carbon emissions is not energy efficiency of the storage technology but rather a shift in the generation mix from less efficient load-peaking units to more efficient intermediate and baseload generator units. The reduced $\mathrm{CO}_{2}$ emissions of $10 \%$ to $20 \%$ are reflective of California's generation mix, and will vary throughout the country as the generation mix changes. More fundamentally, the potential reduction in carbon emissions is directly correlated to the net of carbon intensity of the input fuels for the avoided generation (shifted from) and the new generation (shifted to). Other programs have shown that shifting load from peak load generating power plants to off-peak-load shoulder and base-load power plants that are more efficient and consume less carbon-intensive input fuels results in energy and $\mathrm{CO}_{2}$ benefits.

\section{E.3 Summary}

\section{E.3.1 Estimation of Energy and Carbon Reductions}

The estimation of energy and carbon benefits achievable by load shifting is challenging because of the highly dynamic nature of the power plant dispatch options that provide literally thousands of options of re-arranging the generation mix, hence generating energy efficiency and carbon intensity of the input fuel, as the load shifts. Models that simulate optimal power plant dispatch are required to provide the necessary insights into these dynamics. But because these models are very complex to set up, particularly when simulating a grid scenario that is operated significantly differently from the current "business as usual" case, we applied a simplified approach. This approach was based on the key assumption that demand response will shift the generation mix from inefficient CTs during peak load periods to higher efficient fossil-fuel powered plants during off-peak-load periods. The higher efficient fossil-fuel powered plants are a combination of natural gas combined-cycle, natural gas steam turbines, and coal steam turbines, based on the availability of existing capacity in the region. Figure E.1 shows the load duration 
curves of 12 North American Electric Reliability Corporation (NERC) sub-regions. Data are based on 2002 system load information for 8760 hours. ${ }^{1}$

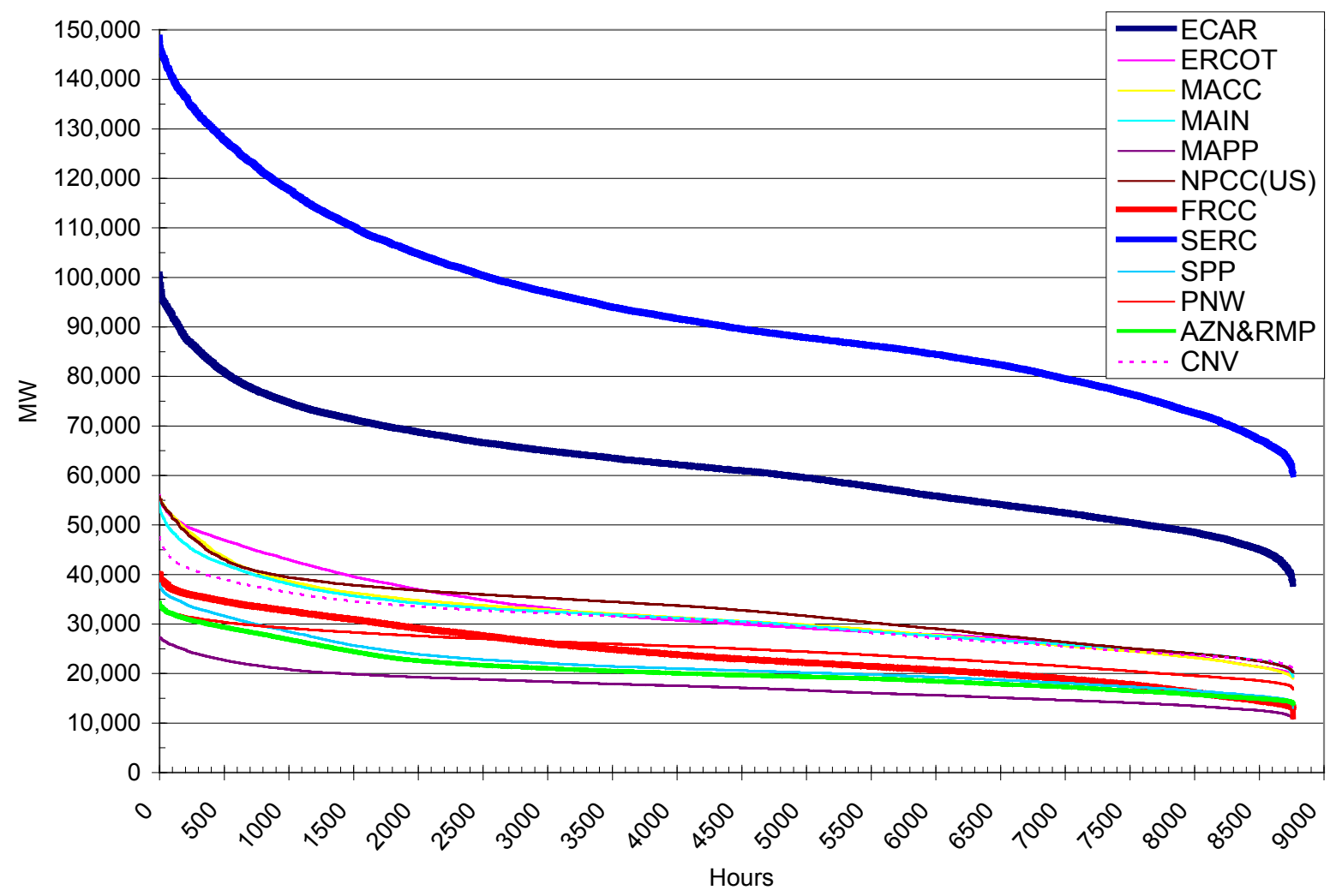

Figure E.1. Load Duration Curves by 12 NERC Sub-Regions (2002)

Following dispatch merit order principles based on cost, CTs will meet the peak loads dispatched as the very last power plant category. This is the generation at the top-left corner of the load duration curve (as seen in Figure E.2). The size of the CT generation "wedge" to be shifted to off-peak-load periods is limited by the demand response or distributed storage capability in each region, assumed to be $10 \%$ of the peak load. The size of the CT generation wedge can then be estimated as the area under the load duration curve, defined by the height corresponding to the installed capacity of CTs or $10 \%$ of peak load, whichever is less (as seen in Figure E.2).

In general, the load is shifted to natural-gas-fired combined-cycle power plants, which reduces energy input and $\mathrm{CO}_{2}$ emissions because of their lower heat rates (higher fuel efficiency). For regions in which there is relatively less combined-cycle capacity and coal-fired power plants predominate (East Central Area Reliability Coordinating Agreement [ECAR], Mid-America Interconnected Network, and MidContinent Area Power Pool), a portion of the energy was shifted to coal-fired power plants based on the judgment of the author. This produces energy savings because of their lower heat rates, but causes higher $\mathrm{CO}_{2}$ emissions because of the higher carbon content of coal compared to natural gas.

The net results of the displacement size of CT generation and its substitution by off-peak-load power are shown in Table E.1.

\footnotetext{
${ }^{1}$ Data were obtained from NERC 2/24/2006.
} 


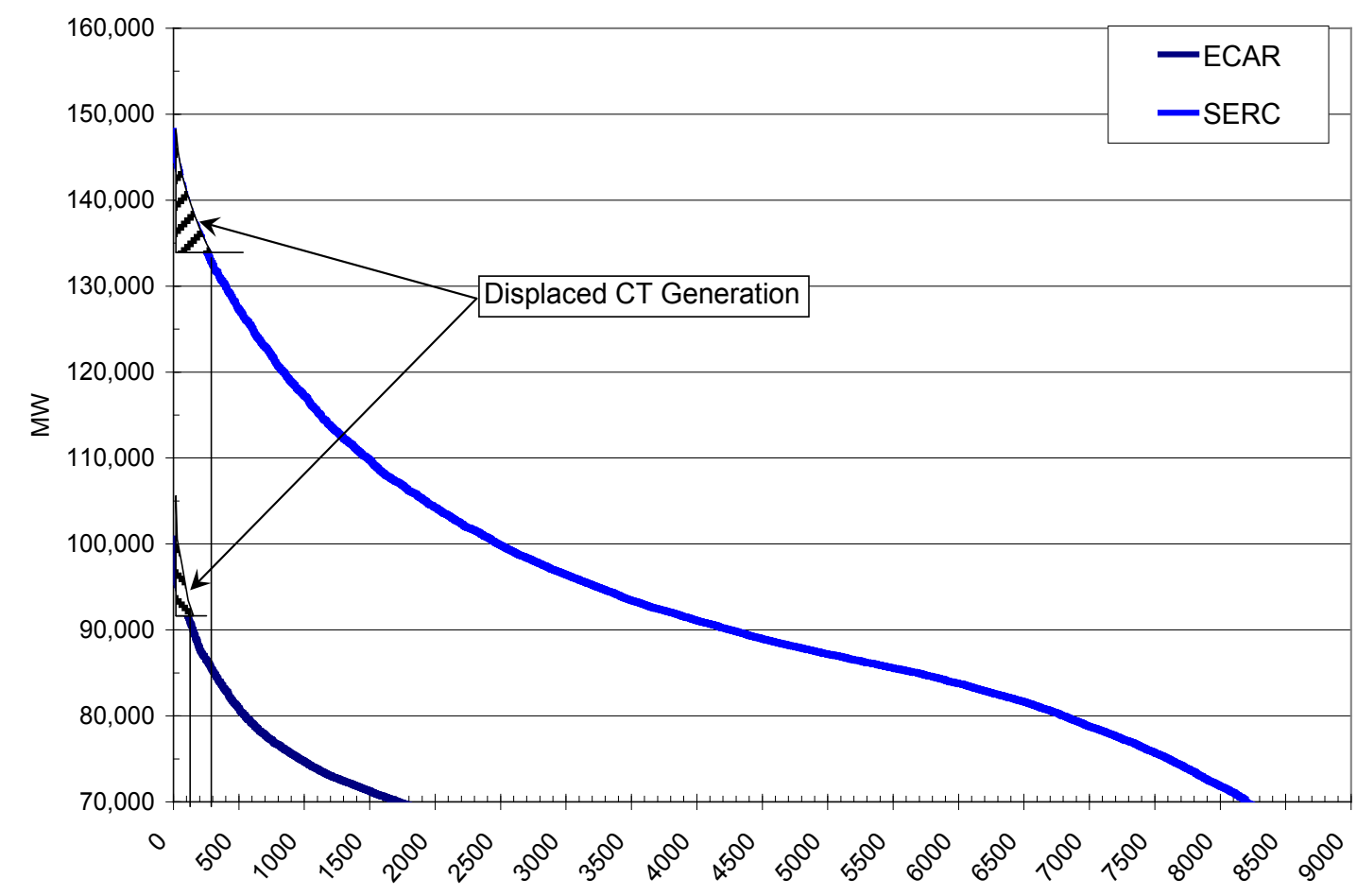

Figure E.2. Approach of Determining the Displaced CT Energy: Load reduction (MW) limited by total installed capacity of CTs or assumed load-shifting capability of $10 \%$ of peak load, whichever is less.

Table E.1. Results of Substituting CT Generation with Off-Peak-Load Generation as a Result of Load Shifting

\begin{tabular}{|c|c|c|c|c|c|c|c|c|c|}
\hline & $\begin{array}{l}\text { Installed } \\
\text { CT } \\
\text { capacity } \\
\text { [GW] }\end{array}$ & $\begin{array}{l}\text { Reduced } \\
\text { CT } \\
\text { capacity } \\
\text { [GW] }\end{array}$ & $\begin{array}{c}\text { Displaced } \\
\text { CT } \\
\text { Generation } \\
\text { [GWh] }\end{array}$ & $\begin{array}{c}\text { Duration of CT } \\
\text { Generation } \\
\text { Displacement } \\
\text { [h] }\end{array}$ & $\begin{array}{c}\% \text { of displaced } \\
\text { energy } \\
\text { provided by } \\
\text { comb. cycle } \\
\text { (nat. gas) }\end{array}$ & $\begin{array}{c}\% \text { of } \\
\text { displaced } \\
\text { energy } \\
\text { provided by } \\
\text { steam turbine } \\
\text { (nat. gas) }\end{array}$ & $\begin{array}{c}\% \text { of } \\
\text { displaced } \\
\text { energy } \\
\text { provided by } \\
\text { steam turbine } \\
\text { (coal) }\end{array}$ & $\begin{array}{c}\text { Energy } \\
\text { Savings } \\
\text { relative to } \\
\text { Displaced } \\
\text { CT } \\
\text { Generation } \\
{[\%]}\end{array}$ & $\begin{array}{c}\text { CO2 savings } \\
\text { relative to CT } \\
\text { displaced } \\
\text { generation } \\
{[\%]}\end{array}$ \\
\hline ECAR & 19 & 10 & 433 & 135 & $50 \%$ & & $50 \%$ & $23 \%$ & $-10 \%$ \\
\hline ERCOT & 5 & 5 & 246 & 142 & $100 \%$ & & & $35 \%$ & $35 \%$ \\
\hline MACC & 10 & 6 & 395 & 167 & $100 \%$ & & & $35 \%$ & $35 \%$ \\
\hline MAIN & 16 & 5 & 156 & 81 & $60 \%$ & $40 \%$ & & $24 \%$ & $24 \%$ \\
\hline MAPP & 6 & 3 & 242 & 209 & $20 \%$ & & $80 \%$ & $16 \%$ & $-37 \%$ \\
\hline NPCC(US) & 7 & 6 & 334 & 145 & $100 \%$ & & & $35 \%$ & $35 \%$ \\
\hline FRCC & 10 & 4 & 243 & 206 & $100 \%$ & & & $35 \%$ & $35 \%$ \\
\hline SERC & 29 & 15 & 1,510 & 276 & $100 \%$ & & & $35 \%$ & $35 \%$ \\
\hline SPP & 7 & 4 & 214 & 173 & $100 \%$ & & & $35 \%$ & $35 \%$ \\
\hline PNW & 2 & 2 & 86 & 149 & $100 \%$ & & & $35 \%$ & $35 \%$ \\
\hline AZN\&RMP & 6 & 3 & 252 & 230 & $100 \%$ & & & $35 \%$ & $35 \%$ \\
\hline CNV & 6 & 5 & 174 & 101 & $100 \%$ & & & $35 \%$ & $35 \%$ \\
\hline US & 124 & 68 & 4,284 & & & & & $32 \%$ & $26 \%$ \\
\hline
\end{tabular}

The energy and carbon savings estimates are based on the improved utilization of off-peak-load generation capacity and reduced utilization of on-peak-load CT resources. The reductions in electricity and $\mathrm{CO}_{2}$ emissions calculated in Attachment 2 are based on this analysis, additional information from the literature review, and the author's experience. It is estimated that a direct reduction of $0.04 \%$ in total 
electricity supplied to the grid, with a range of $0.02 \%$ to $0.06 \%$, and $0.03 \%$ reduction in $\mathrm{CO}_{2}$ emissions (approximately $75 \%$ of the electricity reduction) can be can be achieved through implementation of smart grid technologies. These reductions need to be seen in the context that the overall generation displacement was relatively small (about $0.1 \%$ of the total generation) during short period of time (168 hours on average). No indirect reductions in electricity or capacity are expected.

\section{E.4 Recommendations}

The estimation of energy and $\mathrm{CO}_{2}$ reductions varies widely due to the types of power plants that provide base, intermediate, and peak load power, and the order for dispatch. It is recommended that the estimates of energy and $\mathrm{CO}_{2}$ reductions that result from load shift be subjected to a more methodical effort to determine differences that may result by minimizing economic impacts, maximizing energy reductions, and maximizing $\mathrm{CO}_{2}$ reductions.

\section{E.5 Reference}

Cypress Ltd. (Cypress). 2007. New Program Announcement: Shift \& Save. Accessed December 29, 2009, at http://www.shiftnsave.com/pge/pdf/cyp_shiftsave_program102207.pdf. 


\section{Mechanism F: Support Additional Electric Vehicles and Plug-In Hybrid Electric Vehicles}

\section{F.1 Introduction}

There is a synergetic relationship between electrifying the vehicular transportation sector and the ability of the grid to generate and deliver the "electric" fuel to millions of electric vehicles (EVs) and plug-in hybrid electric vehicles (PHEVs) (Kintner-Meyer et al. 2007). The new load resulting from the PHEV is acyclic to the existing load served, meaning that with load-management strategies (discussed below) the new vehicle load can be served during the off-peak-load night-hour period. The resulting benefit would be improved utilization of the electric infrastructure (generation, transmission, and distribution assets) from the increased sale of electricity with no additional investment in infrastructure (Scott et al. 2007).

An $E V$ has only an electric motor that receives its energy from a battery that must be plugged into an outlet to recharge. A PHEV has both an electric motor that receives its energy from a battery and an internal combustion engine, and the battery can be charged by either plugging it into an outlet or internal combustion engine and/or braking. In each, the battery is sized such that the electric energy is sufficient to cover a typical daily commute of about 40 miles.

The relevant question within the context of smart grid technology is how advanced load-management technologies improve the overall grid efficiency and reduction of carbon emissions compared to unmanaged charging of the emerging EV fleet. These improvements would be in addition to the fuelswitching benefits and inherent efficiency improvements as the vehicle fleet replaces gasoline-fueled internal combustion engines with electric motors.

If EV load-management strategies can be effectively implemented, additional benefits could be realized as we are building out the future power plant fleet. The emerging EV load fundamentally changes the type of future-generation capacity built to serve future load growth by filling in the nocturnal valley and counteracting the growing daytime peak load caused by the growth in air-conditioning load (GE 2008). This flattening of the daily load profiles favors base-load capacity, thereby having significant implications for investment in electric infrastructure, fuels needed for electricity generation, and the emissions from the generating resources that will be in place for the next 40-plus years.

In addition, advanced smart charging strategies of EVs could meet some of the growing ancillary services requirements as more intermittent renewable resources are integrated into the U.S. power grid.

\section{F.2 Review}

Publications in the open literature related to impacts of PHEVs on the grid and the environment have significantly increased since the State of the Union Address by President Bush in January 2006. Universities, National Laboratories, think-tanks, and interest groups have been publishing articles and position papers focusing on the oil displacement opportunities that articulate and quantify the energy security benefits of electrifying vehicles, as well as climate change, and air-quality impacts. All of these assessments use a model-based approach to capture the impacts on the electric infrastructure and the performance characteristics of the new PHEVs and EVs. 
Assessments found in the literature vary in scope. The scope can be as narrowly defined as only addressing the impacts of PHEVs for a single state or utility service territory. Letendre investigated the impacts of PHEVs for the state of Vermont (Letendre and Watts 2008). Parks studied the impacts of PHEVs on XCEL Energy's service territory in Colorado (Parks et al. 2007). Significant work has been done for California (Cheng et al. 2008; Papavasiliou et al. 2008; Farrell et al. 2007), primarily driven by the Alternative Fuels Plan legislated by Assembly Bill 1007 (Olson et al. 2007; California State Assembly Bill 1007 2005). Most analyses discuss the impacts from a U.S. national perspective (EPRI/NDRC 2007; Hadley and Tsvetkova 2008; Voelcker 2009; Lilenthal and Brown 2007; Markel et al. 2006; Farrell et al. 2007; Kintner-Meyer et al. 2007; Shiau et al. 2009; Wise et al. 2009; EPA 2008; Draper et al. 2008).

The literature on international studies is much sparser. Canada and Japan have performed impact assessments particularly focusing on oil-displacement opportunities (Brandon et al. 2008; Hori 2007). The more comprehensive study was performed for Germany. The impacts of 1 and $20 \mathrm{M} \mathrm{EVs}$ on the German grid for the 2020 and 2030 timeframe were investigated. The study concluded that electrification of transportation has a clear petroleum displacement potential; however, the $\mathrm{CO}_{2}$ emission reduction potential, given the strong contribution by coal to the German generation mix, remains marginal. Only if additional "green" generation technologies are brought online could the transportation electrification achieve major $\mathrm{CO}_{2}$ reduction (Horst, 2009).

The majority of the U.S. studies used performance characteristics of PHEVs were compiled from a set of workshops conducted by EPRI in the early 2000s (Duvall 2002, 2003, 2004). Data from real-world performance of PHEVs in operation on the road are currently being collected and compiled by the Idaho National Laboratory.

The key national studies are the Pacific Northwest National Laboratory (PNNL) technical potential assessment of PHEVs based on today's grid (Kintner-Meyer et al. 2007), the Oak Ridge National Laboratory (ORNL) study assessing the impacts on a 2030 projection (Hadley and Tsvetkova 2008), and ERPI/NDRC's (2007) comprehensive study projecting vehicles' penetrations out to 2050:

- The PNNL study indicated carbon reduction potential of $27 \%$ for the nation as a whole with every conventional gasoline vehicle with average fleet fuel economy of $20.7 \mathrm{mpg}$ replaced by a PHEV operating in electricity mode only. For the worst-case condition, a coal-rich region, such as the MidContinent Area Power Pool, in which the marginal generation for charging PHEVs comes almost entirely from coal resources, the well-to-wheel ${ }^{1} \mathrm{CO}_{2}$ emissions are approximately equal to that of conventional vehicles.

- The ORNL study compared PHEVs against a modern hybrid electric vehicle (HEV) for two future grid scenarios (2020 and 2030). ORNL indicated that the $\mathrm{CO}_{2}$ emission benefits were mixed and dependent upon the power level $(120 \mathrm{~V}$ versus $240 \mathrm{~V})$ and the timing and duration that the PHEVs are charged. Based on assumptions, including the time of charging, there could be a net benefit or a loss. The study indicated that from a $\mathrm{CO}_{2}$ emissions perspective charging in the evening could result in a slight net benefit while charging at night would result in a net increase in $\mathrm{CO}_{2}$ emission from PHEVs compared to HEVs.

\footnotetext{
${ }^{1}$ Well-to-wheel emissions are the total emissions associated with the extraction of the primary energy (coal, oil, natural gas, etc.) resource, its transportation, as well as all conversions to usable energy products, including the final conversion into useful end-use energy. The final end-use product in this case is vehicle miles traveled (VMTs).
} 
- The EPRI/NDRC study performed a scenario analysis that defined three possible $\mathrm{CO}_{2}$-intensity paths (high, medium, low). For these scenarios, the study determined emission projections for 2010 and 2050. For the 2010 results, the study concluded that PHEVs will reduce the greenhouse gas (GHG) emissions by $28 \%$ to $34 \%$ compared to conventional vehicles, even in the case when all electricity is supplied by coal-fired power plants currently in operation. The GHG emissions of a PHEV would be $1 \%$ to $11 \%$ higher dependent upon battery size when compared to HEVs. These results are consistent with the ORNL study and, by extension, consistent with the PNNL study as well. For the 2050 cases, however, the study estimated the PHEV GHG emissions to be lower compared to even a HEV for all $\mathrm{CO}_{2}$ intensity scenarios.

These national studies all suggest that the $\mathrm{CO}_{2}$ benefits highly depend on the reference vehicle to which a PHEV or EV will be compared (more on this subject below). Furthermore, the results indicate that the timing and the duration of the charging influence the carbon intensity. Absent any new clean base-load capacity, the marginal generation at night will, to a great degree, remain coal-based with high carbon emissions.

On an energy-per-VMTs basis, EVs, in general, have a higher total efficiency by about $30 \%$ above that for conventional vehicles (See Table F.1) Argonne National Laboratories' GHGs, regulated emissions, and energy use in transportation (GREET). The energy conversion in the vehicle itself is clearly more efficient with electric motors (efficiencies are in the range of $70 \%$ to $80 \%$. Full-trip efficiencies of batteries (full-charge/full-discharge cycle) is in the $80 \%$ range, yielding EV efficiencies in the mid to upper $60 \%$ range. This is about twice the efficiency of internal combustion energy, which is in the mid to upper $20 \%$ range.

Table F.1. Energy and $\mathrm{CO}_{2}$ Emissions Benefits Using the GREET Model, 2001

\begin{tabular}{|c|c|c|c|c|c|c|c|c|c|c|c|c|c|}
\hline & ECAR & ERCOT & MACC & MAIN & MAPP & NPCC & FRCC & SERC & SPP & PNW & N\&RI & CNV & \begin{tabular}{|c|} 
US \\
total \\
\end{tabular} \\
\hline & \multicolumn{12}{|c|}{ Power Generation Composition } & \\
\hline Natural Gas & $32 \%$ & $94 \%$ & $74 \%$ & $42 \%$ & $1 \%$ & $91 \%$ & $69 \%$ & $57 \%$ & $78 \%$ & $43 \%$ & $63 \%$ & $93 \%$ & \\
\hline Coal & $68 \%$ & $6 \%$ & $26 \%$ & $58 \%$ & $99 \%$ & $9 \%$ & $31 \%$ & $43 \%$ & $22 \%$ & $57 \%$ & $37 \%$ & $7 \%$ & \\
\hline Energy & \multicolumn{13}{|c|}{ Energy Ratio (Electric Vehicle/Gasoline Vehicle) } \\
\hline Total Energy & 0.71 & 0.68 & 0.70 & 0.71 & 0.73 & 0.69 & 0.70 & 0.70 & 0.69 & 0.71 & 0.70 & 0.69 & 0.70 \\
\hline Emissions & \multicolumn{12}{|c|}{ Emissions Ratio (Electric Vehicle/Gasoline Vehicle) } & \\
\hline $\mathrm{CO} 2$ & 0.87 & 0.60 & 0.69 & 0.83 & 1.01 & 0.61 & 0.71 & 0.76 & 0.66 & 0.84 & 0.73 & 0.61 & \\
\hline
\end{tabular}

\section{F.2.1 Implementation Requirements and Process for Smart Grid Application}

The smart grid implementation to support EV technology involves one strong and one weak or optional requirement:

Strong requirement: Load-management technology and strategies, sometimes referred to as "smart charging" technologies, are a key requirement to guard against the emerging EV load to be coincident with the system peak load, and with local and regional transmission and distribution bottlenecks. Loadmanagement technologies and strategies will be needed to schedule the vehicle load, based on pricing signals coupled with customer preferences that may influence the load schedule, and by allowing overrides and setting constraints as to when the vehicle will need to be fully charged. The availability of 
price signals sent to customers is a necessary requirement for smart charging strategies; however, they not sufficient. Vehicles or charging stations need to be equipped with communication technologies to receive price signals or other signals to determine or influence the charging schedule. In addition, there needs to be consumer acceptance of this technology to fully utilize its effectiveness.

Furthermore, a wide dissemination of the communication technology requires standardization of communication protocols to provide uniformity across the nation and even the North American continent. The infrastructure requirement for smart charging does not require any technology specific to transportation. It could leverage all the smart grid investments made for residential and commercial customer demand response applications.

Optional requirement: The new emerging load could provide significant benefit as a spinning reserve resource, freeing up generator capacity for energy production. This spinning reserve would be delivered as load resource. It will not require that electricity be fed from the battery back into the grid. The spinning reserve resource could be implemented either with communication utilizing the infrastructure (mentioned above) as a strong requirement. An alternative approach that does not require communication to the load, is a frequency-based approach in which load shedding occurs automatically when the voltage is under-frequency. Implementation of the under-frequency load shedding requires a utility-grade frequency sensor and a controller device1. The new load lends itself to be utilized as a spinning-reserve resource because the events are generally short in duration (15 min or less) and will not noticeably impact the battery-charging process.

\section{F.3 Summary}

This topic examines how advanced load-management technologies through smart charging can improve the overall grid efficiency and reduce carbon emissions compared to unmanaged charging of the emerging EV fleet. The marginal benefits due to the managed charging of EVs and PHEVs enabled by smart grid technology will provide reductions in ancillary services, load management, and reductions in energy consumption and $\mathrm{CO}_{2}$ emissions. These improvements would be in addition to the fuel-switching benefits and inherent efficiency improvements as the vehicle fleet replaces gasoline-fueled internal combustion engines with electric motors. National studies suggest that the $\mathrm{CO}_{2}$ benefits depend highly on the reference vehicle to which a PHEV or EV will be compared (more on this subject below). The results indicate that the timing and duration of the charging influence the carbon intensity, and that emission reductions may be as high as a $\sim 30 \%$ net increase.

\section{F.3.1 Energy and Carbon Reductions}

EV transportation fundamentally requires more energy from the grid, not less, as electricity displaces fossil transportation fuel (primarily gasoline). There are inherent efficiency benefits when electric motors replace gasoline engines. To capture these efficiency benefits, one has to consider the inter-sectoral shift in the energy requirements from transportation fuel to electricity and then estimate the energy benefits resulting from this fuel switch. The energy benefits are estimated from a well-to-wheel perspective that

\footnotetext{
${ }^{1}$ PNNL developed a control technology for this application. More information can be obtained at: http://gridwise.pnl.gov/docs/pnnlsa36565.pdf
} 
considers the entire energy conversion path from the extraction of primary energy to the final conversion into useful end-use energy (VMTs in this case).

The energy and carbon benefits of smart grid technology applied to EVs reflect, then, the degree to which we can utilize the efficiency improvements associated with the fuel and motor substitution process. In an unmanaged EV charging scenario, the size of the EV fleet that can be supported by the existing grid will be limited or smaller when compared to the EV fleet applying smart grid load control technologies. The smart grid energy and carbon benefits are then estimated as the difference in the improvements between a larger EV fleet (requiring smart grid technology) and a smaller fleet (left unmanaged). It should be clearly noted that this simplified approach will only credit smart grid benefits until the EV fleet size reaches its limit set by unmanaged charging. As will be discussed below, that limit is fairly high, meaning that the grid can accommodate many millions of EVs and PHEVs at the national level without load-management strategies. This simplified approach neglects the economic benefits of off-peak-load charging, even for a small number of EVs and PHEVs, by utilizing higher efficient intermediate or baseload power plants. For reasons discussed in the Caveats section below, the simplified approach was used because of the complexity of an optimal power plant dispatch analysis that provides sufficient detailed data on the energy requirements and emissions between different charging scenarios.

\section{F.3.2 Estimation of Benefits for Fuel Substitution in Vehicular Transportation}

The challenge for the net benefit estimation strongly depends on the definition of the conventional gasoline technology against which the EV is compared. The literature has discussed this quite extensively, with several suggestions for defining the base-case vehicle:

- the fuel economy of today's on-the-road fleet of light-duty vehicles (LDVs), which currently is about $20 \mathrm{mpg}$ (EIA 2008a), reasoning that the new EV purchased in the near term would, on average, replace an existing vehicle represented by the average fuel economy

- the corporate average fuel economy standard vehicle, which per EISA 2007 was increased to $35 \mathrm{mpg}$ in 2020 (Public Law 110-140). The argument was put forth that this figure would best represent the competition of a comparable new vehicle to be purchased by 2020 or perhaps before that time.

- a vehicle that represents a highly fuel-efficient configuration, such as a gasoline-hybrid electric midsized vehicle with a fuel economy of $43 \mathrm{mpg}$ or a turbo-charged direct-injection diesel mid-size car with an fuel efficiency of about $40 \mathrm{mpg}$ (Wang 2001). These figures were argued to represent most realistically the competition of EVs and, thus, should be taken as the conventional vehicle against which to compare the impacts of EVs.

At this point, it will not be suggested which of the three base cases is the most appropriate to use for this analysis, as it may depend on the particular policy questions to be considered. However, it should be noted that the higher the fuel economy of the conventional vehicle the higher the bar for EVs to achieve energy and emissions benefits.

\section{F.3.3 Benefit Assessment Approach}

The approach used for this effort is analogous to the methodology described in Kintner-Meyer et al. (2007). It is based on a valley-filling approach that estimates the idle generation capability of the existing grid to support an emerging PHEV customer segment that could be utilized for charging the PHEV/EV batteries. It is the generation that is available after all the existing non-transportation load has been 
satisfied, which is considered the marginal generation. Applying this approach and adopting assumptions stated in Kintner-Meyer et al. (2007), the marginal generation is provided by two power plant types: 1) coal steam power plants and 2) natural gas combined-cycle power plants.

The marginal generation by power plant type is input into the GREET model (Wang 2001). The model output provides the improvement in energy and emissions from a well-to-wheel perspective. The

analysis is performed for the 12 modified North American Reliability Corporation (NERC) sub-regions to reflect the varying electric generation mix for charging the batteries. The results in Table F.1 reflect the improvements expressed as a ratio relating to energy requirements and emissions of electric to conventional gasoline vehicles. The reference (conventional gasoline) vehicle represents the fuel efficiency of today's average mid-size car (Wang 2001). The results indicate that if you replace a conventional vehicle with an EV, then the energy improvement per VMT improves by $30 \%$ (the energy ratio is 0.70 ) as a weighted national average. Similarly, the $\mathrm{CO}_{2}$ emission improves by $27 \%$.

\section{F.3.4 Unmanaged Versus "Smart" Charging}

The estimates of vehicles that can be supported by the regional grid are a technical potential that requires "perfect valley-filling" load-management strategies such that the nocturnal valley is filled out without setting a new system peak load. To achieve "perfect valley-filling" requires smart grid technologies that carefully sequence the charging of PHEVs and EVs. The question is what the maximum percentage of the LDV fleet in the absence of load management would be if, for instance, owners of PHEVs would plug in their vehicles at home or at work at the time they arrive. We analyzed the 2001 National Household Travel Survey data to determine the arrival and departure times for travel to and from home and work, and the miles traveled between those destinations (NHTS 2001). We assumed that the PHEV will be plugged into a power supply and charge until either the PHEV is used for the next trip or when the battery is fully charged. Assuming a 120V/12A charger, with battery sizes varying corresponding to the vehicle class composition with an all-electric range of 33 miles, we estimated the charging profiles and the new resulting system load for the technical potential of the regional PHEV fleet.

Figure F.1 below shows the results of both the "perfect valley-filling" of the entire regional fleet, as well as the new system load for the regional PHEV fleet if plugged in at home immediately after arriving, based on the NHTS statistics when people arrive home. Unlike in the "perfect valley-filling" solution, which avoided a new system peak load, permitting unmanaged PHEV charging may set some new system peak loads in several regions. Constraining the system load such that it never exceeds the peak load demand on peak load days reduced the technical potential of the number of vehicles that could be supported by the grid from $73 \%$ to $64 \%$ of the entire U.S. LDV fleet (Kintner-Meyer et al. 2008).

The reductions in electricity and $\mathrm{CO}_{2}$ emissions calculated in Attachment 2 are based on this and additional information from the literature review, and the author's experience. It is estimated that managed charging provides for a direct reduction of 3\% in energy consumption by enabling $9 \%(18 \mathrm{M})$ more EVs to be entered into the fleet with a $30 \%$ reduction in energy consumed per VMT. No indirect reductions in electricity or capacity are expected. 


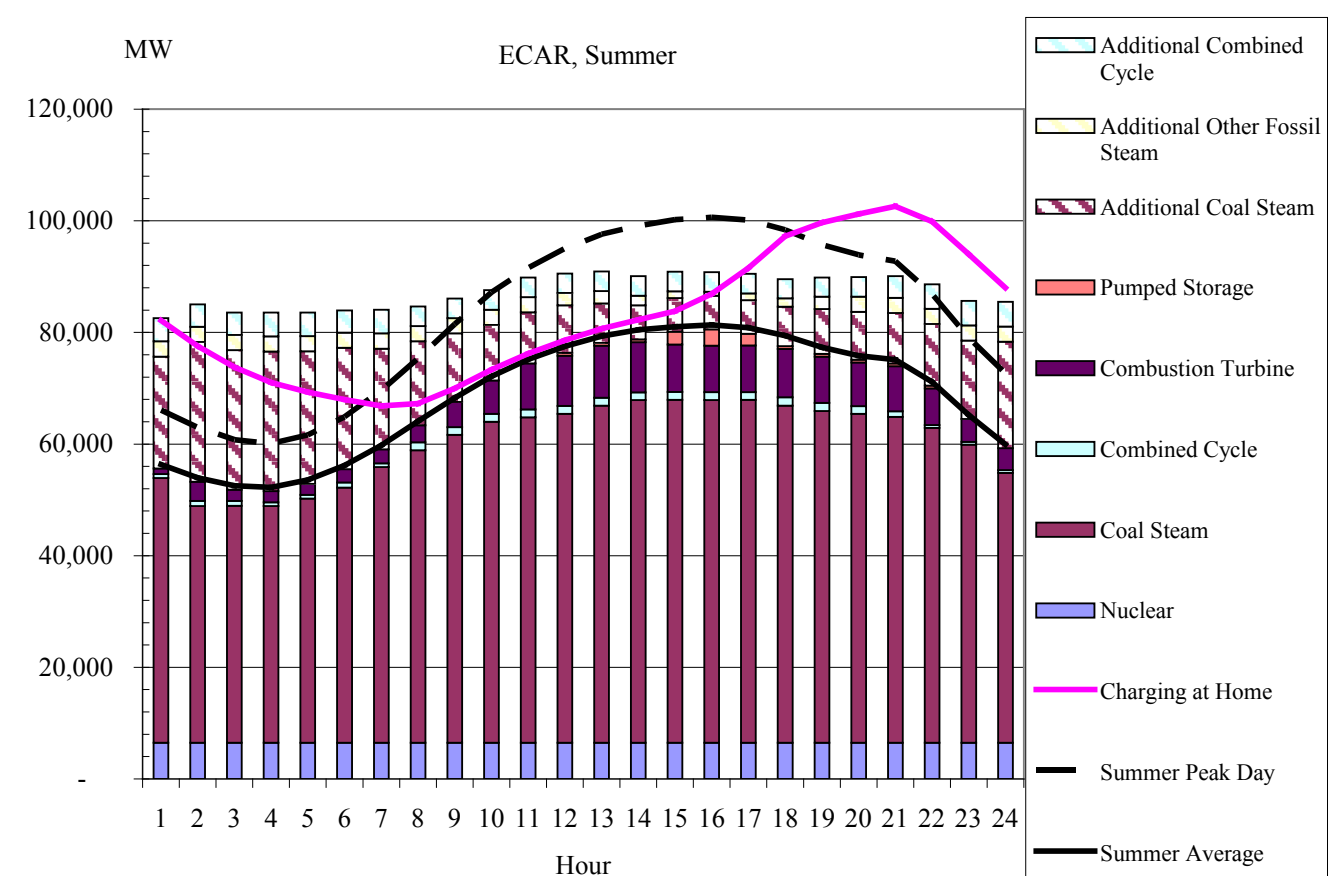

Figure F.1. ECAR Dispatch for Summer Average Load Profile, Valley-Filling Potential, Peak Load Day, and Estimated System Load When Charging a) at Home, b) Home and Work

\section{F.3.5 Caveats of the Simplified Approach}

The benefit estimation is predicated on the assumption that the electric vehicle fleet (EVs and PHEVs) will grow to a size above $64 \%$ of our LDV stock. In absolute numbers of vehicles, it would amount to about 140 million cars, SUVs, pickup trucks, and vans (DOT 2002). It is not clear if and when EVs will have reached such a market penetration. In other words, there is no timeline associated with the realization of the smart grid benefits. This makes it very difficult to define the reference scenario for a conventional car. In other words, is the reference car a conventional car in 2020 or 2050? Beyond the 2020 time horizon, it becomes increasingly difficult to determine the performance characteristics of a conventional car.

Furthermore, the simplified benefit estimation quantifies the enabling characteristics of smart grid technologies by relaxing the bound from $64 \%$ to $73 \%$ of the LDV stock in the United States, allowing an additional 18 million EVs in the national fleet. The benefits of 18 million vehicles should only be seen as a bounding estimate or technical potential.

There is currently much discussion about whether 120 -volt charging will be the norm. In large vehicles like SUVs, charging at that voltage for a 30-mile range can take 12 hours or more. Shorter charging times may be highly desired by consumers, and 240-volt charging may become the standard. 240 -volt outlets in garages of new homes are already required in California for this reason. To a first order, charging at 240 volts doubles the peak load impact of unmanaged charging, and therefore cuts the number of vehicles that can be supported with unmanaged charging in half, to about 70 million. This reduces the fraction of electric VMT that can be supported by the grid before smart charging is required to $32 \%$, raising the smart grid's impact from $9 \%$ to $41 \%$. 
The simplified analysis neglects the economic benefits of off-peak-load charging, even for a small number of EVs and PHEVs, by utilizing higher efficient intermediate or base-load power plants. To capture the benefits of smart versus unmanaged charging requires a comprehensive modeling effort that determines the economic dispatch of power plants with security-constraint unit commitment. With such a tool, the difference in the power plant dispatch between dumb and smart charging can be assessed. However, the results will be strongly dependent on the assumption of the fleet size and assumptions of future capacity expansions. Because of significant uncertainties as to how EVs and PHEVs will penetrate the market-place in the future, in addition to uncertainties associated with future state and federal renewable portfolio standards directives, a comprehensive economic power plant dispatch analysis is beyond the scope of this effort.

\section{F.4 Recommendations}

Because of the significant limitation of the simplified analysis it is recommended to replace the simplified approach with a more comprehensive analysis that analyzes the incremental benefits of loadmanagement strategies in even small but growing EV fleets. This analysis should be done for several years in the future to capture the changing characteristics of our power plant fleet imposed by RPS mandates as well as the growing load imposed by a growing EV fleet. The fundamental tool for performing such analyses has been developed by PNNL in a recent analysis that estimates the impacts of PHEV on wholesale power markets. A report is forthcoming. The results will not only be of significance to this section, but also to other smart grid technologies that impact load shapes.

\section{F.5 References}

Brandon R, E Entchev; T Takeda, H Ribberink, and M Charal. 2008. Part I: Analysis of Plug-in Hybrid Vehicle Performance. Part II: Methodology for Investigating the Impacts of PHEV Charging Scenarios on Cost and Environmental Impact. Final Report. Natural Resources Canada. CETC-O Project IES MG 012. Ottawa, Ontario, Canada.

California State Assembly Bill 1007. 2005. California State Assembly, Sacramento, California.

Cheng I, D Desai, K Koudigkelis, P de Vasconcellos, P Kaminsky, I Sidhu, and B Tenderich. 2008. Electric Vehicle Charging Infrastructure Rollout Strategy. Technical Brief No. 2008.1.v.1. University of California-Berkeley, Global Venture Laboratory, Berkeley, California. Accessed December 29, 2009, at http://cet.berkeley.edu/dl/EV1Rollout Final.pdf

DOT - U.S. Department of Transportation. 2002. Highway Statistics 2001. Table 5-1: Motor-Vehicle Registrations: 2001. U.S. Department of Transportation, Federal Highway Administration, Washington, D.C.

Draper M, E Rodriquez, P Kaminsky, I Sidhu, and B Tenderich. 2008. Economic Impact of Electric Vehicle Adoption in the United States. Technical Brief No. 2008.3.v.1. University of CaliforniaBerkeley, Global Venture Laboratory, Berkeley, California. 
Duvall M. 2002. Comparing the Benefits and Impacts of Hybrid Electric Vehicle Options for Compact Sedan and Sport Utility Vehicles. EPRI -1006892, Electric Power Research Institute, Palo Alto, California.

Duvall M. 2003. "Electricity as an Alternative Fuel: Rethinking Off-Peak Charging." In: Plug-in HEV Workshop. Electric Power Research Institute, Palo Alto, California.

Duvall M. 2004. Advanced Batteries for Electric Drive Vehicles: A Technology and Cost-Effectiveness Assessment for Battery Electric Vehicles, Power Assist Hybrid Electric Vehicles, and Plug-in Hybrid Electric Vehicles. EPRI-1009299, Electric Power Research Institute, Palo Alto, California. Accessed December 29, 2009, at http://mydocs.epri.com/docs/public/000000000001009299.pdf.

EIA - U.S. Energy Information Administration. 2008a. "Reference Case, Table 35: High Technology Matrix for Cars." In: Assumptions to the Annual Energy Outlook 2008 with Projections to 2030. DOE/EIA-0383(2008), U.S. Government Printing Office, Washington, D.C.

EPA - U.S. Environmental Protection Agency. 2008. "The Potential Energy and GHG Implications of Plug-in Hybrid Vehicles: A Scenario Analysis." Presented at the Government/Industry Meeting, May 14, Washington D,C.

EPRI/NRDC - Electric Power Research Institute/National Resources Defense Council. 2007. Environmental Assessment of Plug-In Hybrid Electric Vehicles. Volume 1: Nationwide Greenhouse Gas Emissions. EPRI, Palo Alto, California. Accessed December 29, 2009, at http://my.epri.com/portal/server.pt?open=512\&objID=243\&PageID $=223132 \&$ cached $=$ true $\&$ mode $=2$

Farrell AE, D Lemoine, and DM Kammen. 2007. "Effect of Plug-in Hybrid Electric Vehicles in California Energy Markets." In: The $86^{\text {th }}$ Annual Meeting of the Transportation Research Board, Paper No. 07-3497. January 21-25, National Academy of Sciences, Washington, D.C.

General Electric Company (GE). 2008. "Power of Knowledge: Enabling the Intelligent Grid." GE Energy Seminar, July 15, 2008. GE, Schenectady, New York. Accessed December 29, 2009, at http://www.ist.co.za/data/Presentations_Day2/The\%20Smart\%20Home_IST\%20Energy.pdf.

Hadley ST and AA Tsvetkova. 2008. Potential Impacts of Plug-In Hybrid Electric Vehicles on Regional Power Generation. ORNL/TM-2007/150, Oak Ridge National Laboratory, Oak Ridge, Tennessee.

Hori M. 2007. Plug-in Hybrid Electric Vehicles for Japan: Opportunities, Effects, Efficiencies, and Barriers. Universal Energy Research Institute, Tokyo, Japan.

Horst Juri, Günther Frey,and Uwe Leprich. 2009. Auswirkungen von Electroautos auf den Kraftwerkspark und die CO2-Emissions in Deutschland. World Wildlife Fund, Deutschland. Frankfurt, Germany. Accessed December 29, 2009, at http://www.wwf.de/downloads/publikationsdatenbank/ddds//1//5/.

Kintner-Meyer M, K Schneider, and R Pratt. 2007. "Impact Assessment of Plug-In Hybrid Vehicles on Electric Utilities and Regional U.S. Power Grids. Part 1: Technical Analysis." Journal of EUEC, 1: Paper No. 04. http://www.euec.com/documents/pdf/Paper_4.pdf. 
Kintner-Meyer M., R Pratt, and K Schneider. 2008. "Comprehensive Well-to-Wheel Analysis for Plugin-Hybrid-Electric-Vehicles in the U.S.” In: Proceedings of the International Federation of Automotive Engineering Societies (FISTA) World Automotive Congress, September 14-19, Paper Number F2008-09-011, Munich, Germany.

Letendre S and RA Watts. 2008. Effects of Plug-In Hybrid Electric Vehicles on the Vermont Electric Transmission System. University of Vermont, Transportation Research Board, Burlington, Vermont. Accessed December 29, 2009, at http://www.uvm.edu/ transctr/trbpapers/Effects_of_PHEVs_on the Vermont_Electric_Transmission System.pdf.

Lilienthal P and H Brown. 2007. "Potential Carbon Emissions Reductions from Plug-In Hybrid Electric Vehicles by 2030.” In: Tackling Climate Change in the U.S., CF Kutscher, Editor, pp. 69-77. American Solar Energy Society, Boulder, Colorado.

Markel T, M O'Keefe, A Simpson, J Gonder, and A Brooker. 2006. Plug-In HEVs: A Near-Term Option to Reduce Petroleum Consumption. NREL/540-MP-39415, National Renewable Energy Laboratory, Golden, Colorado. Accessed December 29, 2009, at http://www.nrel.gov/docs/fy06osti/39415.pdf

National Household Travel Survey (NHTS). 2001. User's Guide. U.S. Department of Transportation, Washington, D.C.

Olson T, M Addy, and D Schwyzer. 2007. State Alternative Fuels Plan: Commission Report. CEC-6002007-011-CMF. California Air Resources Board; California Energy Commission, Sacramento, California. http://www.energy.ca.gov/2007publications/CEC-600-2007-011/CEC-600-2007-011CMF.PDF.

Papavasiliou A, A Lee, P Kaminsky, I Sidhu, B Tenderich, and S Oren. 2008. Electric Power Supply and Distribution for Electric Vehicle Operations. Technical Brief No. 2008.2.v.1. University of California-Berkeley, Global Venture Laboratory, Berkeley, California. Accessed December 29, 2009, at http://cet.berkeley.edu/d1/EV2Grid Final.pdf.

Parks K, P Denholm, and T Markel. 2007. Costs and Emissions Associated with Plug-In Hybrid Electric Vehicle Charging in the Xcel Energy Colorado Service Territory. NREL/TP-640-41410, National Renewable Energy Laboratory, Golden, Colorado. Accessed December 29, 2009, at http://www.nrel.gov/docs/fy07osti/41410.pdf.

Public Law 110-140, as amended. 2007. Energy Independence and Security Act of 2007, $110^{\text {th }}$ Congress, Washington, D.C.

Scott MJ, M Kintner-Meyer, DB Elliot, and WM Warwick. 2007. "Impact Assessment of Plug-In Hybrid Vehicles on Electric Utilities and Regional U.S. Power Grids. Part 2: Economic Assessment." Journal of EUEC 1: Paper No. 05. Accessed December 29, 2009, at http://www.euec.com/documents/pdf/Paper 5.pdf. 
Shiau CN, C Samaras, R Hauffe, and JJ Michalek. 2009. "Impact of Battery Weight and Charging Patterns on the Economic and Environmental Benefits of Plug-In Hybrid Vehicles." Energy Policy 37(7):2653-2663.

Voelcker J. 2009. “How Green is My Plug-In?” IEEE Spectrum 46(3):42-58.

Wang MQ. 2001. Development and Use of GREET 1.6 Fuel-Cycle Model for Transportation Fuels and Vehicle Technologies. ANL/ESD/TM-163, Argonne National Laboratory, Argonne, Illinois.

Wise MA, GP Kyle, JJ Dooley, and SH Kim. 2009. "The Impact of Electric Passenger Transportation Technology on the Demand for Coal-fired Power with CCS under a Climate Policy." Energy Procedia 1(1):4355-4362. 



\section{Mechanism G: Conservation Voltage Reduction and Advanced Voltage Control}

\section{G.1 Introduction}

Traditionally, distribution utilities have operated under the premise that they have no control over the amount of power consumed by the end users. This has forced the design of a power generation and delivery system that is focused on being able to meet the peak load system demand-a goal that is achieved at substantial cost. Additionally, in an economically competitive environment older, less efficient generating units are more likely to be run when there is a high demand for power, increasing the system's overall emissions. Since the 1980 s, it has been postulated that it is possible for a utility to lower the voltage on a distribution feeder to reduce energy consumption (Lauria 1987). The basic premise is that electrical loads draw less power at lower voltages, consuming less power and reducing line losses because of the reduced load. Another benefit is that if this reduction is performed during peak load, then it is less likely that older, less efficient generating units would need to be run, due to the load reduction. The process of operating a distribution system at a lower voltage to reduce energy consumption is referred to as conservation voltage reduction (CVR).

While there are examples of distribution utilities that have implemented CVR systems, they have done so without many of the technologies that constitute the smart grid. In the absence of a strong communications infrastructure and controllable devices on the feeder, these utilities have controlled the voltage reduction only at the substation. While this operational strategy has yielded measureable reductions in energy consumption, both on- and off-peak load, smart grid technologies could further improve these reductions in energy consumption and $\mathrm{CO}_{2}$ emissions through advanced monitoring and control strategies.

\section{G.2 Review}

\section{G.2.1 The CVR Effect}

When the concept of CVR was initially proposed, there was considerable debate about its validity. The reason for the skepticism was that not all electrical loads respond to voltage changes in the same way. A simple electrical load, such as an incandescent light bulb or resistive heating element, will consume less power when the voltage is reduced. A load such as an induction motor could draw the same amount of power but will draw more current, which will result in higher line losses. Therefore, the effectiveness of CVR is a function of the loads connected to the distribution feeder. The result is that feeders in regions that utilize resistive residential heating will behave differently from feeders in regions that utilize heat pumps.

Another factor to take into account is whether the energy consumed was truly conserved or just deferred when voltage is reduced; this is a major concern with thermostatic loads. Resistive heating elements controlled by a thermostat will consume less power when the voltage is lower, thus producing less heat, but will remain on longer to achieve the desired heating set point. The net result is that while the peak load power was reduced, the same amount of energy was consumed. While the same amount of power may be consumed, distributing it over a longer time may mitigate the need to run less efficient 
generating units, thus reducing the overall emissions. So even when there is no net reduction in load, there are potential capacity and emission reductions. The net benefit of CVR is dependent on the system design, load composition, and generation mix. Analytically determining the aggregate effect on a distribution feeder for a given reduction in voltage is a non-trivial task that cannot be determined with existing commercial software packages.

\section{G.2.2 Deployed CVR Examples}

In the absence of effective analytic techniques, numerous distribution utilities have taken on the cause of CVR and implemented CVR systems to empirically evaluate the operational benefits. Two of the first utilities to systematically examine the operational impact of CVR, and publish the results, were the Snohomish Public Utility District (PUD) in Everett, Washington (Kennedy and Fletcher 1991), and Commonwealth Edison in Chicago, Illinois (Kirshner 1990). In both cases, the feeder voltage was lowered at the substation to a level that ensured that the most remote load on the feeder would be within the requirements of ANSI C84.1 (ANSI 1996).

The primary metric for CVR is the CVR savings factor (CVRf), which is a ratio of the percent change of energy consumption for a given percent change in voltage level $(\% \mathrm{E} / \% \mathrm{~V})$ (Fletcher and Saeed 2002). Positive values of CVRf indicate that the system is consuming less energy as the voltage is lowered and a negative value indicates the opposite. In both cases, (Kennedy and Fletcher 1991; Kirshner 1990), it was found that the CVRf was in the range of 0.5 to 1.0 and the net energy consumed by the feeders was reduced. For Snohomish PUD where the average feeder has 12,500,000 MWh of load, a reduction of 2.1 volts, with a CVRf of 0.621 yields energy savings of $162,500 \mathrm{kWh} /$ year per feeder (Kennedy and Fletcher 1991). If the generation unit is fueled by natural gas, approximately 211,250 pounds of carbon dioxide per feeder is displaced per year. To determine the total carbon dioxide reduction for the entire Snohomish PUD service area a detailed breakdown of their generation mix is required.

The most comprehensive evaluation of CVR is discussed in the "Distribution Efficiency Initiative 2007 Report" prepared for the Northwest Energy Efficiency Alliance (Beck 2007). In this report, 11 distribution utilities in the Pacific Northwest participated in a two-part study to determine the effectiveness of CVR.

The first part of the study was a load research project and the second part was a series of pilot demonstration projects. The load research project involved installing 395 load-regulation devices at the point of connection for residential customers. The reason for doing this was to determine the exact CVRf values for individual residences and compare these values to those determined at the substation. The result was that there were discrepancies between the values of CVRf determined at the residences, 0.569 , and at the substation, 0.690 . The discrepancy is due to the differences in electrical characteristics between the electrical load and the distribution system. Accurate determination of the CVR effects must include analysis of the electrical load as well as the design of the distribution system. The design of the distribution feeders includes everything from line and cable types, line and cable configurations, use of voltage correction capacitors, and use of tap-changing voltage regulators for transformers. The second part of the study involved the analysis of multiple CVR pilot projects that utilized methods for voltage control. The method of voltage control was often determined by the hardware capabilities of the utilities. The two most common methods of voltage control were line-drop compensation and active monitoring and control of an end-point voltage. Line-drop compensation is a relatively low-cost method of ensuring 
that the system voltage is maintained within limits. The system involves controlling the voltage at the substation so that the voltage at the far point of the system is within limits, without directly monitoring the remote voltage. Instead, the voltage at the substation is measured and based on an internal model, and feeder loading the voltage at the far end it estimated. While this is a lower cost option, it does not always give the correct answer, thus large margins of error are assumed. In an active monitoring system, voltage transducers are place at remote ends of the feeder. While this is a more expensive method, it is much more accurate and allows for smaller margins of error.

In total, 31 feeders at 10 different substations participated in the second part of the study. When the average 15 -minute peak load demand reductions were examined voltage reductions ranged from $1.02 \%$ to $3.51 \%$ and CVRf ranged from $0.25 \%$ to $1.30 \%$. The highest CVRf did not correlate to the large percentage reduction in voltage. This lack of correlation can be attributed to variations in feeder design and operational methodologies.

The existing body of work has shown that there are tangible energy savings that can be obtained from a properly implemented CVR system (Kennedy and Fletcher 1991; Kirshner 1990; Beck 2007; Wilson 2002). The extent to which CVR can be utilized will depend on the load types as well as the design and construction of the distribution feeders. These energy savings can be translated directly into reduced emissions, particularly $\mathrm{CO}_{2}$ (EPRI 2008).

\section{G.2.3 Limitations of Existing CVR Systems}

Most utilities maintain the voltage supplied to their consumers within the guidelines set by ANSI C84.1, which is $120 \mathrm{~V}+/-5 \%, 114 \mathrm{~V}-126 \mathrm{~V}$ (ANSI 1996). To ensure that the customers near the end of the feeder are within this range it is common to raise the substation voltage to a higher level. Figure G.1 shows the voltage drop on a radial distribution feeder for peak load and minimum load (note: the voltage drop is a function of both the feeder load and length). From Figure G.1 it can be seen why it is necessary to set the substation voltage to a higher level. If the voltage at the substation was set at the middle of the band, $120 \mathrm{~V}$, then the customers at the end of the feeder would be below the acceptable range during the peak load. Figure G.1 shows a system that would not need any adjustments at the substation as the system moves from minimum load to peak load.

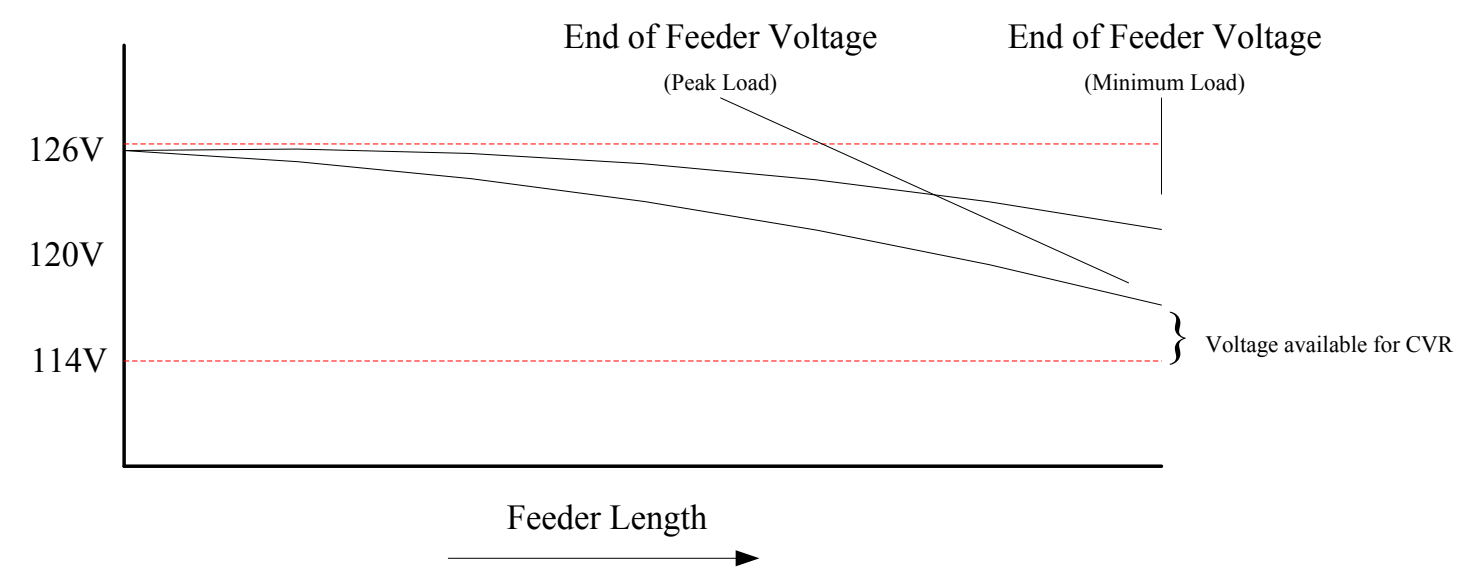

Figure G.1. Passive Voltage Drop 
For a heavily loaded feeder or a long rural feeder, it may not be possible to maintain the proper voltage by adjustments of the substation voltage alone. As a result, many distribution feeders utilize voltage regulators or shunt capacitors to maintain voltage levels at proper levels. This allows for a system to sustain more electrical load before it is necessary to either shift load to adjacent feeders, or to reconductor the feeder to provide higher current levels and defer capital expenses. Figure G.2 shows an example of a radial distribution feeder that has a very large voltage drop. To accommodate the large voltage drop, a shunt capacitor is placed approximately $60 \%$ down the length of the line. This has the effect of reducing line losses and voltage drop along the feeder. To compensate for the variation in voltage throughout the day, shunt capacitors can be automatically switched in and out based on a local voltage measurement.

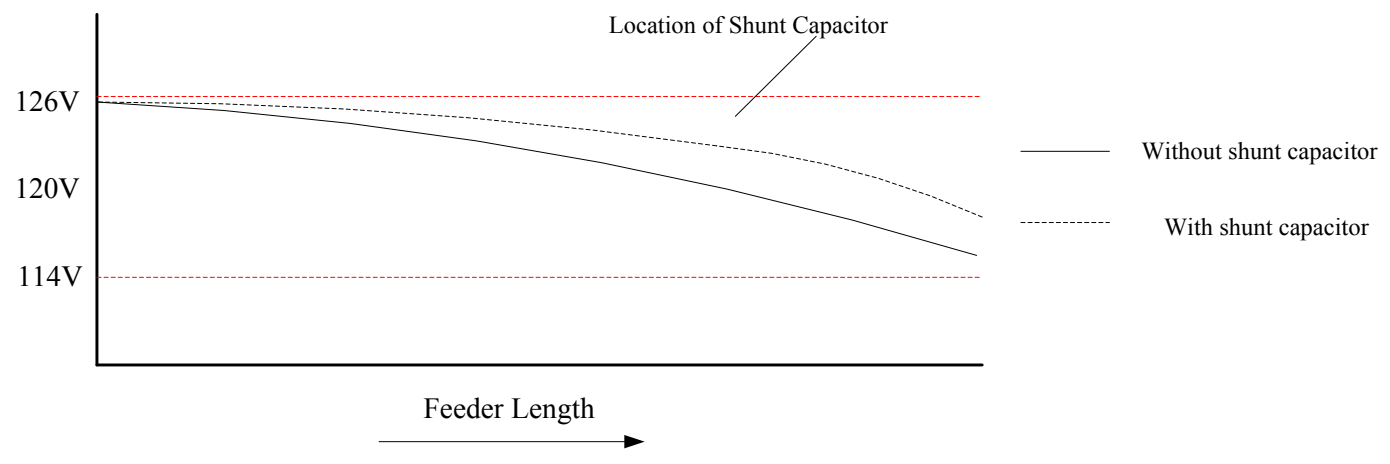

Figure G.2. Capacitor Compensated Voltage Drop

While CVR can, and has been, implemented on feeders with automatically switched shunt capacitors, their limitation is that they cannot always respond to changing load conditions. As a result, they are not adequate for leveling the feeder voltage enough for aggressive CVR, i.e., more than a 2-volt reduction. To achieve voltage reduction of greater than one or two volts, the development of additional techniques to enhance CVR will be necessary.

\section{G.3 Summary}

CVR has empirically proven itself to be a viable method to reduce the peak load on a distribution feeder as well as being an effective form of conservation. Empirical work reviewed showed that a 1\% change in distribution line voltage provided a $0.25 \%$ to $1.3 \%$ change in energy consumption. Accurate determination of the CVR effects must include analysis of the electrical load as well as the design of the distribution system. The design of the distribution feeders includes everything from line and cable types, line and cable configurations, use of voltage correction capacitors, and use of tap-changing voltage regulators for transformers.

Using existing CVR technologies, it is possible to reduce the current consumption of electricity by approximately $1 \%$ with little or no capital investment. Deploying smart grid technologies could potentially increase this $3 \%$ to $4 \%$, which translates directly into reduced energy consumption and $\mathrm{CO}_{2}$ emissions. The reductions in electricity and $\mathrm{CO}_{2}$ emissions calculated in Attachment 2 are based on this and additional information from the literature review, and the author's experience. It is estimated that a direct reduction of $2 \%$ in total electricity supplied to the grid, with a range of $1 \%$ to $4 \%$, can be achieved through implementation of smart grid technologies. No indirect reductions in electricity or capacity are expected 


\section{G.4 Recommendations for Additional Work and/or Consideration}

Significant additional research is needed to determine to what extent CVR can be exploited and to determine more about its fundamental nature. Currently the majority of knowledge is empirical and cannot be validated analytically. The consequences of this is that we do not fully understand how new technologies will interact with CVR and thus we cannot fully exploit them.

If there are multiple capacitors on the feeder and they are properly coordinated, it is possible to maintain a much more "flat" voltage profile (McCarthy and Josken 2003). With a flat voltage profile, it is possible to lower the voltage to a much lower level at the substation. Figure G.3 shows such an example. Figure G.3 shows that the voltage profile will only allow for a reduction of substation voltage of approximately one volt without the presence and operation of shunt capacitors. In this case, multiple capacitors with coordinated control can allow for a reduction of substation voltage of several volts. An additional benefit to the use of coordinated capacitor controls is that the lower voltages are seen at all points on the feeder, not just near the end. This will result in a higher aggregate CVRf. While this type of control has not been implemented on an actual system, papers have been published describing this type of control system (McCarthy and Josken 2003).

To deploy a coordinated capacitor control scheme that operates on multiple feeders, possibly supplied by the same transformer, it is necessary to implement a form of intelligent control. It is in this role that the smart grid can significantly contribute, with its near-real-time sensing, monitoring, and control capability. A fully functional smart grid would enable CVR schemes to interact with demand response and distributed energy resources to provide improved system operation with energy, capacity, and emission benefits.

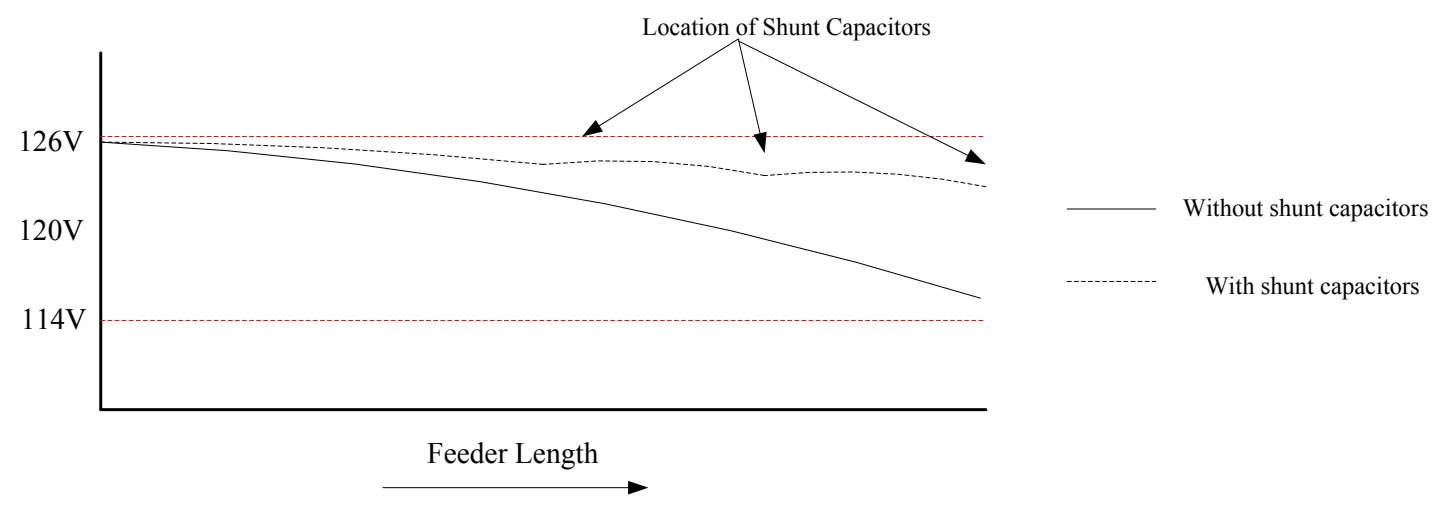

Figure G.3. Coordinated Capacitor-Controlled CVR

\section{G.5 References}

American National Standards Institute (ANSI) Standard C84.1. 1996. "American National Standard for Electric Power Systems and Equipment-Voltage Ratings (60 Hertz).” National Electrical Manufacturers Association, Rosslyn, Virginia.

Beck RW. 2007. Distribution Efficiency Initiative. Technical Report prepared for the Northwest Energy Efficiency Alliance, Portland, Oregon. 
Electric Power Research Institute (EPRI). 2008. The Green Grid: Energy Savings and Carbon Emissions Reductions Enabled by a Smart Grid. ERPI-1016905. EPRI, Palo Alto, California.

Fletcher RH and A Saeed. 2002. "Integrating Engineering and Economic Analysis for Conservation Voltage Reduction.” In: IEEE Power Engineering Society Summer Meeting, Vol 2, pp. 725-730. July 25, Everett, Washington. Institute of Electrical and Electronics Engineers, Piscataway, New Jersey.

Kennedy BW and RH Fletcher. 1991. "Conservation Voltage Reduction (CVR) at Snohomish PUD." IEEE Transactions on Power Systems 6(3):986-998.

Kirshner D. 1990. "Implementation of Conservation Voltage Reduction at Commonwealth Edison." IEEE Transactions on Power Systems 5(4):1178-1182.

Lauria DM. 1987. "Conservation Voltage Reduction (CVR) at Northwest Utilities." IEEE Transactions on Power Delivery 2(4):1186-1191.

McCarthy CA and J Josken. 2003. "Applying Capacitors to Maximize Benefits of Conservation Voltage Reduction." In: Rural Electric Power Conference, pp. C4-1-C4-5, May 4-6, Raleigh-Durham, North Carolina. Institute of Electrical and Electronics Engineers, Piscataway, New Jersey.

Wilson TL. 2002. "Energy Conservation with Voltage Reduction-Fact or Fantasy." In: IEEE Rural Electric Power Conference, pp. C3-C3_6. May 5-7, 2002, Colorado Springs, Colorado. Institute of Electrical and Electronics Engineers, Piscataway, New Jersey. 


\section{Mechanism H: Support Penetration of Solar Generation (RPS $>25 \%$ )}

\section{H.1 Introduction}

Solar photovoltaic (PV) power is one of the most recognizable forms of renewable energy. The ability to convert sunlight directly into electricity with zero emissions, excluding manufacturing and disposal of the solar cells and associated equipment, led many people to believe that it is an ideal solution to the growing demand for electricity (Kroposki et al. 2008). In the 1960s and 1970s, when the cost of solar PV was still very high, it was only used in applications were an interconnection to the electricity infrastructure was very expensive or impractical. These applications included satellites, navigation aids, and remote highway indicators. Starting in the 1980s, the prices for solar PV began to lower to the point where they could be installed at residential locations, although they were still relatively expensive. Since 2000, the price of solar PV arrays has dropped to the point where they are starting to become economically competitive, especially with the aid of tax credits.

If this trend continues as expected, solar PV will become an economically viable method for the distributed production of power in the near future (Kroposki et al. 2008). When this occurs on large scales, it will be conceivable that electric distribution feeders will have numerous solar PV arrays feeding power back into the system, possibly reversing the flow of power on the entire feeder (Ueda et al. 2005a, b). While this has significant social benefits, in the form of reduced emissions, the distribution systems that exist today were not designed to operate in this manner.

\section{H.2 Review}

\section{H.2.1 Power Production with Solar PV}

Individual solar PV cells can be electrically connected in series and/or parallel to deliver the desired voltage and current. This can range from a small single cell on a calculator, to a multiple-array rooftop application, all the way to a large-scale utility application. In areas with significant sunlight, such as the American Southeast, solar PV has the potential to produce significant amounts of energy. Incident solar energy at sea level is approximately $1 \mathrm{~kW} / \mathrm{m}^{2}$ on a clear day. Assuming $20 \%$ cell efficiency and a $90 \%$ conversion efficiency to alternating current (AC), this results in approximately $180 \mathrm{~W} / \mathrm{m}^{2}$ of useable power as an upper limit. This number is reduced in higher latitudes and areas that do not have clear weather. While this may reduce the total usable power to tens of watts per square meter, this technology can be deployed over hundreds or thousands of square meters, resulting in significant amounts of power.

In 2008, Pacific Gas and Electric Company (PG\&E) announced that it would sign an agreement for $800 \mathrm{MW}$ of central solar PV power (PG\&E 2008). This is an example of a large installation similar to a wind farm and, as such, it will be interconnected to the transmission or sub-transmission system. While large central installations will require analysis to integrate them into the existing transmission infrastructure, much more work will be required to integrate solar PV into distribution feeders.

Regardless of the scale of the solar PV array the individual cells produce direct current (DC) power, which must be converted to AC, via an inverter, for use with residential appliances or to feed back into 
the transmission or distribution system (Thomson and Infield 2007)). The conversion from DC to AC adds significant cost to a solar PV installation, especially in residential applications where economies of scale cannot be exploited. Additionally, a utility may specify additional hardware requirements for distributed resource applications. For example, IEEE 1547 states in section 4.1.7 that a utility can require "a readily accessible, lockable, visible-break isolation device shall be located between the Area Electric Power System and the distributed resource unit" (IEEE 2008). Even with these costs solar PV deployed in distributed resource applications are expected to increase because of the benefits of clean power, both perceived and actual.

For utilities, the potential for a single distribution feeder to have several hundred solar PV installations feeding back into the feeder is a significant concern. Operating in this manner would violate many of the assumptions that were made when the system was built. One of the largest concerns is the potential voltage rise that could occur with solar PV inverters feeding back into the feeders (Ueda et al. 2005a, 2005b) and (PG\&E 2008). Because the voltage is assumed to drop continually on a distribution feeder (Kersting 2007), it is common practice to set the voltage high at the substation. If the voltage setting at the substation is not coordinated with the output of the various solar PV inverters it is possible to have an over- or under-voltage condition that violates the limits set forth in ANSI (1996).

Because the output of solar PV can change faster than the voltage control devices at the substation, especially during cloud transients, there is a maximum operational limit to the amount of solar PV that can be integrated using existing technologies. The existing voltage regulators at substations are electromechanical devices that operate on an order of seconds. In comparison, the output of a solar PV array can go from full to zero in well under a second. Voltage-correction capacitors can operate in less time, but they are not generally coordinated to work with installed generation. As a result, it will be necessary to deploy new technologies to allow for significant solar PV penetration levels.

\section{H.2.2 Voltage Profiles Issues}

Utilities normally maintain the voltage supplies to the customer between 114V and 126V in accordance with (ANSI 1996). To ensure that all loads on the feeder receive a voltage within this range, the voltage at the substation is set to the high end of the range and the voltage drops over the length of the

feeder. In the absence of devices that produce real or reactive power, the voltage drops continually until it reaches the end of the feeder. Figure H.1 shows two voltage profiles, minimum load and peak load. In both cases the voltage drops continually, but the drop is more pronounced during the peak load condition. 


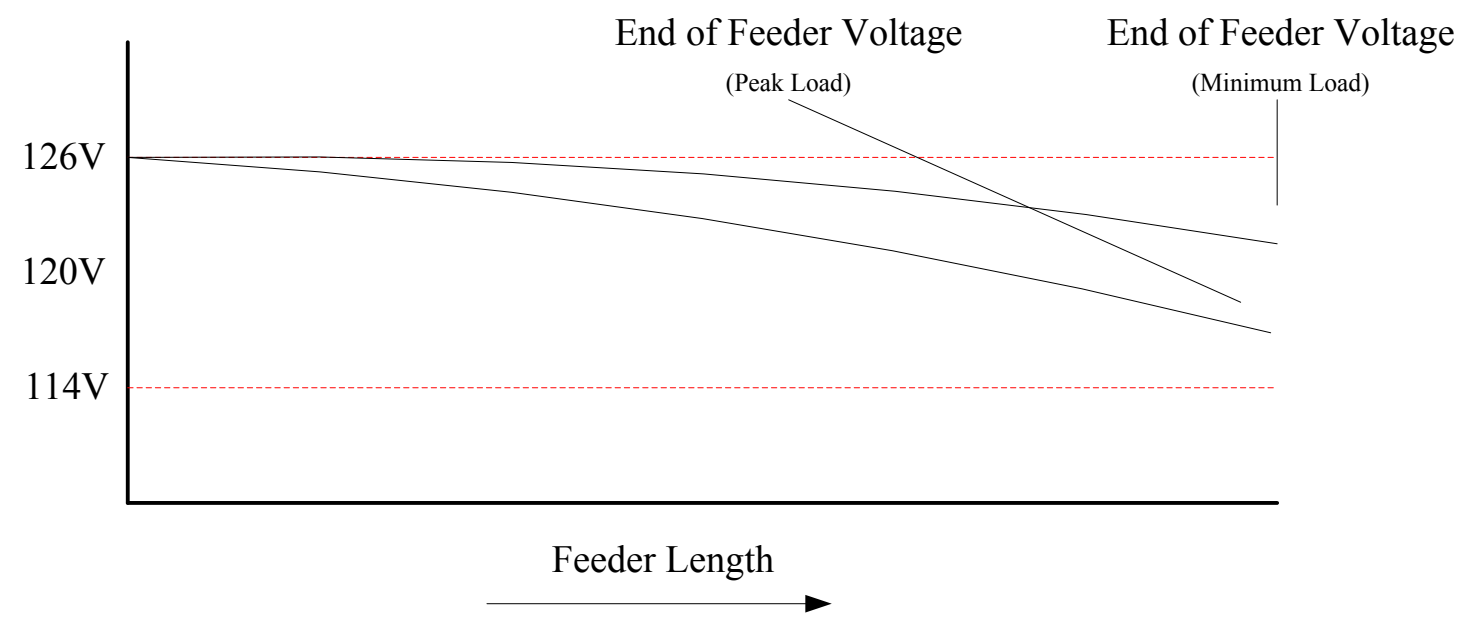

Figure H.1. Typical Distribution Feeder Voltage Profile

If there is a moderate amount of solar PV that causes a small amount of reverse power flow, it generally will not be a problem. If this occurs on a heavily loaded system, the reverse power flow will help to minimize the voltage drop on the feeder (Acquaviva et al. 2000). If the outputs of the inverters are coordinated, the profile can be further improved.

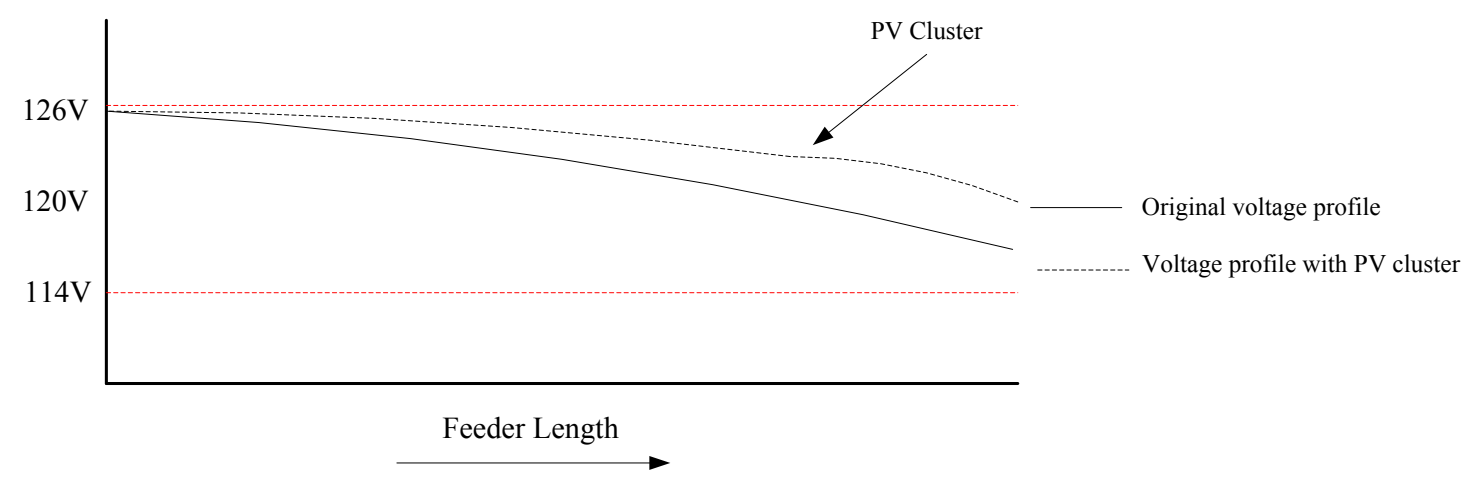

Figure H.2. Distribution Feeder Voltage Profile With a Single Cluster of Solar PV

If there is a large commercial or industrial installation of solar PV (PG\&E 2008) or multiple large commercial or residential installations, then it is possible for the voltage profile to move outside the limits of ANSI (1996). Additionally, if there are extensive clusters of solar PV, then some voltage levels could be high when there is no cloud cover and low when there is cloud cover. Attempting to have the substation regular account for this would result in numerous operations of the regulator and frequent voltage excursions. 


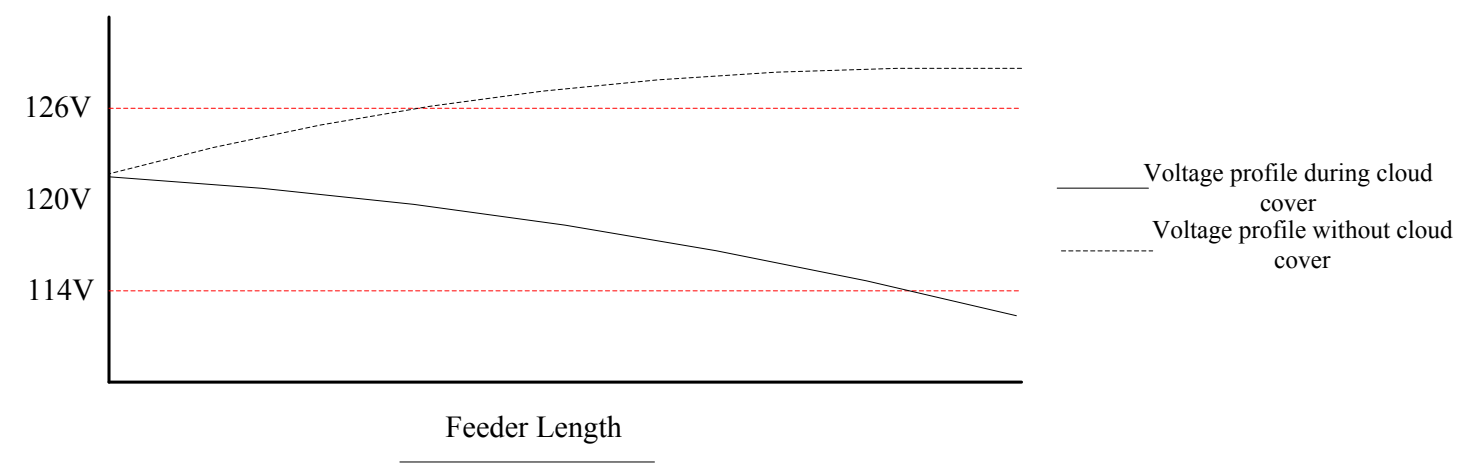

Figure H.3. Distribution Feeder Profile With Large Clusters of Solar PV With and Without Cloud Cover

\section{H.2.3 Estimation of the Limits to Solar PV Penetration}

To estimate how much solar generation can exist in a typical residential neighborhood before reverse power flow can be expected, we examine a worst-case scenario. This consists of maximum solar output on a perfectly clear day at noon, in the spring or fall when heating and air conditioning are not needed in homes, and in a neighborhood that uses natural gas for water heating (like most in the United States) The total electrical load for a home at noon without heating, cooling, or water heating is about $1 \mathrm{~kW}$ (Pratt et al. 1993.

The average electricity consumption for an average U.S. home in 2001 was $10,656 \mathrm{kWh} / \mathrm{year}$, and the average share by end use is shown Table H.1. ${ }^{1}$

Table H.1. Share of Electricity Consumption by End Use in an Average U.S. Home

\begin{tabular}{|c|c|c|c|c|}
\hline $\begin{array}{c}\text { Space } \\
\text { Heat }\end{array}$ & $\begin{array}{c}\text { Water } \\
\text { Heat }\end{array}$ & $\begin{array}{c}\text { Air } \\
\text { Conditioning }\end{array}$ & Other & $\begin{array}{c}\text { Other + Air } \\
\text { Conditioning }\end{array}$ \\
\hline $10.3 \%$ & $11.4 \%$ & $15.8 \%$ & $62.5 \%$ & $78.3 \%$ \\
\hline
\end{tabular}

These include all types of homes: single-family detached, mobile homes, and apartments, owned and rented. The average annual electricity consumption for a single-family detached owner-occupied home was estimated by multiplying $10,656 \mathrm{kWh} /$ year by the ratio of 1) the total electrical energy consumed in such homes to 2) the total energy consumed for all types of homes in 2005 (DOE/EIA 2005). This produces an estimated consumption of $12,341 \mathrm{kWh} /$ year. To determine the total consumption by end use for the average single-family detached owner-occupied home, the end-use shares in Table H.1 were multiplied by this estimate, with the results shown in Table H.2.

Table H.2. End-Use Electricity Consumption for Average U.S. Single-Family Detached Home $(\mathrm{kWh} /$ year)

\begin{tabular}{|c|c|c|c|c|c|}
\hline Total & $\begin{array}{c}\text { Space } \\
\text { Heat }\end{array}$ & $\begin{array}{c}\text { Water } \\
\text { Heat }\end{array}$ & $\begin{array}{c}\text { Air } \\
\text { Conditioning }\end{array}$ & Other & $\begin{array}{c}\text { Other + Air } \\
\text { Conditioning }\end{array}$ \\
\hline 12,355 & 1,248 & 1,124 & 1,977 & 7,993 & 8,599 \\
\hline
\end{tabular}

\footnotetext{
${ }^{1} 2001$ is the latest year for which such breakdowns by share are available.
} 
These estimates include homes whose space and water heat are provided by all sources, including natural gas, fuel oil, kerosene, propane, and wood. The total electricity consumption for the homes that do not use electricity for space and water heating, the basis for the analysis here, is the sum of the consumption for the Air Conditioning and Other end uses: about 8,600 kWh/year.

Reverse power flow will begin if every home has a solar PV array that is just big enough, on average, to meet the load. On a very clear day (solar clearness index, $\mathrm{KT}=0.75$ ), the incident solar radiation on a place tilted toward the sun at noon ${ }^{1}$ is $75 \%$ of the extraterrestrial solar radiation, or a little over $1 \mathrm{~kW} / \mathrm{m}^{2}$ $\left(1015 \mathrm{~W} / \mathrm{m}^{2}\right)$. If the solar conversion efficiency is $17 \%$, then a net of $6 \mathrm{~m}^{2}$ of solar cells are needed to produce $1 \mathrm{~kW}$ of power.

The daily solar energy varies by location due to latitude and cloud cover. The annual average annual average on a south-facing surface with a tilt equal to the latitude for various locations in the United States is indicated by the color coding on the map in Figure 3.9. To this, we have added a second scaling key to the color coding that reflect the annual average solar renewable portfolio standard (RPS) for this neighborhood at which the output of the solar array on such a day exactly matches the $1 \mathrm{~kW}$ load of the home.

The scaling key is produced corresponding to the original color coding key that has increments of incident daily solar energy shown in the first line of Table H.3, below. This is multiplied by 365 days per year to get the annual incident solar energy in the second line of the table. This is then multiplied by the net active area of solar PV cells in the array, $6 \mathrm{~m}^{2}$. Note that the results here are independent of the assumed conversion efficiency; a lower efficiency simply requires a higher net area that intercepts a correspondingly higher total of incident radiation to produce $1 \mathrm{~kW}$, for example. As the final step, the annual output of the solar PV array is divided by the total consumption of the home for the Air Conditioning and Other end uses from Table H.3 to estimate the fraction of the home's annual energy supplied by the solar PV array.

Table H.3. Fraction of Home's Annual Energy Supplied by a 1-kW Solar PV Array

\begin{tabular}{|l|r|r|r|r|r|r|}
\hline Photovoltaic solar resource $\left(\mathrm{kWh} / \mathrm{m}^{2}\right.$-day) & 6.8 & 6 & 5 & 4 & 3 & 2.2 \\
\hline Photovoltaic solar resource $\left(\mathrm{kWh} / \mathrm{m}^{2}\right.$-yr) & 2,482 & 2,190 & 1,825 & 1,460 & 1,095 & 803 \\
\hline PV output, 1-kW array $(\mathrm{kWh} / \mathrm{yr})$ & 2,519 & 2,222 & 1,852 & 1,482 & 1,111 & 815 \\
\hline Fraction of annual energy from PV & $29 \%$ & $26 \%$ & $22 \%$ & $17 \%$ & $13 \%$ & $9 \%$ \\
\hline
\end{tabular}

From Figure 3.9, the annual fraction of energy generated from such a 1-kW solar array is seen to range from a low of about $17 \%$ to a high of about $28 \%$ over most of the United States (excluding Alaska and parts of the Pacific Northwest). The median for the continental United States appears to be around $21 \%$.

Thus, the onset of reverse power flow appears to be a serious barrier to penetrations of solar PV systems in residential neighborhoods to achieve local RPS levels above about 20\%. A smart grid could help circumvent this barrier by deploying and controlling additional voltage regulators, controlling batteries, and providing adaptive short-circuit protection schemes that adapt to reverse power flow on the

\footnotetext{
${ }^{1}$ If the spring or fall day is at the solar equinox (March 20 or September 22), the tilt of the array is exactly equal to the latitude.
} 
fly. Further details are provided in Attachment 1, Mechanism H. Estimates of potential reductions in electricity and $\mathrm{CO}_{2}$ emissions were not made for this mechanism, and there is not an obvious basis for estimating the indirect benefits of removing a barrier such as this. Further refinement of this crude estimate and creating a way to value it is a recommendation for further analysis.

\section{H.3 Summary}

Solar PV is an attractive approach for obtaining zero-emissions energy production because it readily scales to the needed level. This makes it ideal for applications ranging from relatively small residential rooftop applications to larger commercial and industrial rooftop applications. The existing electricity infrastructure can support a limited penetration of solar PV with the current operating schemes, what that limit is will vary from utility to utility. The smart grid holds the promise of allowing much greater penetrations of solar PV and thus much greater reduction in emissions.

Calculation of the estimated reductions in electricity and $\mathrm{CO}_{2}$ emissions were not made. It is estimated that the reductions would be similar to those made for wind integration as either resource, alone or in combination, could be used to satisfy a $20 \%$ RPS.

\section{H.4 Recommendations for Additional Work and/or Consideration}

Exactly when a distribution feeder transitions from solar PV helping the system, Figure H.2, to solar PV becoming a limiting factor, Figure H.3, cannot be simply stated. At some point, there is a penetration level when the addition of more solar PV will have detrimental impacts on the system. The additional variability of the solar PV generation could even require additional fossil-fuel-powered plants to be run, which would negate the emission reductions of the solar PV arrays. Additional research is needed to determine what the feasible limit is with existing technologies, and to determine new operating strategies that allow for greater penetration of solar PV.

Smart grid technologies could allow for the active adjustment of the voltage profile along the length of the feeder. The voltage could be adjusted through a number of mechanisms, including active control of shunt capacitors and regulators, active control of solar PV inverters to operate outside the unity power factor (Ton et al. 2008), and coordinated control of energy storage devices. Figure H.4 shows a potential smart grid scheme where the voltage profile is automatically maintained within the proper limits. When there is no cloud cover, an energy storage device absorbs energy, acting as a load, thus reducing voltage. When there is cloud cover, the regulator slightly increases voltage at the substation and the energy storage discharges power acting as a generator, thus increasing voltage. 


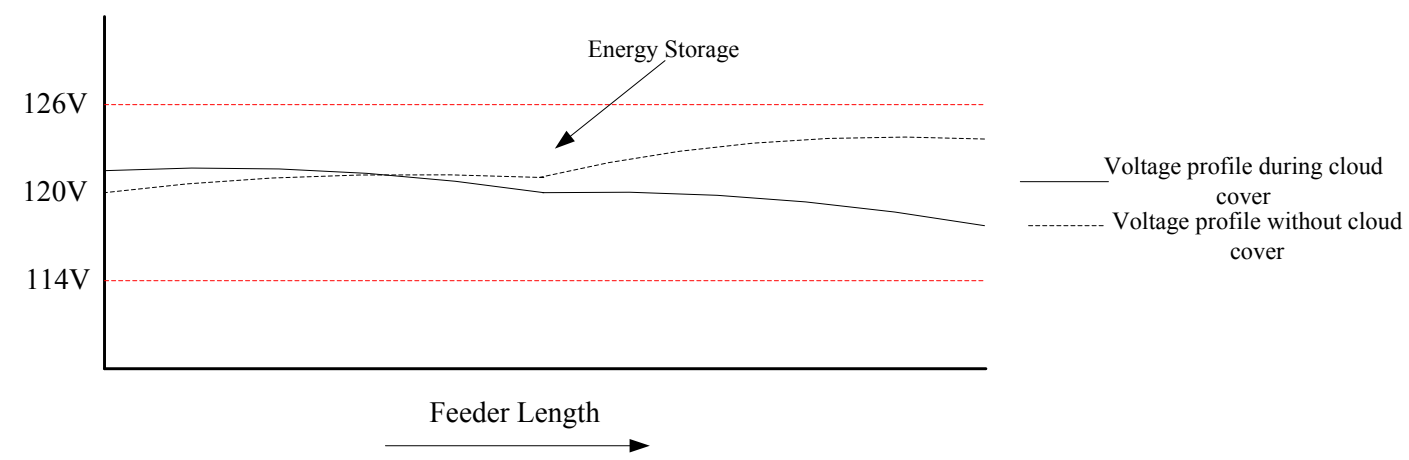

Figure H.4. Distribution Feeder Profile With Large Clusters of Solar PV During and After Cloud Cover With the Interaction of Smart Grid-Controlled Energy Storage

\section{H.5 References}

American National Standards Institute (ANSI) Standard C84.1. 1996. "American National Standard for Electric Power Systems and Equipment-Voltage Ratings (60 Hertz).” National Electrical Manufacturers Association, Rosslyn, Virginia.

Acquaviva V, P Poggi, M Muselli, and A Louche. 2000. “Grid-Connected Rooftop PV Systems for Reducing Voltage Drops at the End of the Feeder-A Case Study in Corsica Island." Energy 25(8):741-756.

DOE/EIA - U.S. Department of Energy/U.S. Energy Information Administration. 2005. Table US-1. Electricity Consumption by End Use in U.S. Households, 2001. Accessed December 29, 2009, at http://www.eia.doe.gov/emeu/reps/enduse/er01_us_tab1.html.

Kroposki B, R Margolis, G Kuswa, J Torres, W Bower, T Key, and D Ton. 2008. "Renewable Systems Interconnection: Executive Summary.” NREL/TP-581-42292, National Renewable Energy Laboratory, Golden, Colorado. Accessed December 29, 2009, at http://www.nrel.gov/eis/publications.html.

Institute of Electrical and Electronics Engineers (IEEE). 2008. "IEEE Standard for Interconnecting Distributed Resources with Electric Power Systems." Standard 1547, IEEE, Piscataway, New York.

Kersting B. 2007. Distribution System Modeling and Analysis. Second Edition, CRC Press, Boca Raton, Florida.

Pacific Gas and Electric Company (PG\&E). 2008. "PG\&E Signs Historic 800-MW Photovoltaic Solar Power Agreements With Optisolar and Sunpower Solar Projects Would Supply Enough Renewable Energy Equivalent to the Energy Needs of 239,000 Californian Homes.” Press Release. PG\&E, New York.

Pratt, RG, CC Conner, BA Cooke, and EE Richman. 1993. "Metered End-Yse Consumption and Load Shapes from the ELCAP Residential Sample of Existing Homes in the Pacific Northwest." Energy and Buildings, Vol. 19, No. 3. Elsevier Science Publishing, New York. 
Thomson M and DG Infield. 2007. "Network Power-Flow Analysis for a High Penetration of Distributed Generation." IEEE Transactions on Power Systems 2(3):1157-1162.

Ton D, CJ Hanley, GH Peek, and JD Boyes. 2008. "Solar Energy Grid Integration System-Energy Storage (SEGIS-ES).” SAND2008-4247, Sandia National Laboratories, Albuquerque, New Mexico and Livermore, California. Accessed December 29, 2009, at http://www.sandia.gov/ess/Publications/SEGIS-ES_SAND2008-4247.pdf.

Ueda Y, T Oozeki, K Kurokawa, T Itou, K Kitamura, Y Miyamoto, M Yokota, H Sugihara, and S Nishikawa. 2005a. "Detailed Performance Analyses Results of Grid-Connected Clustered PV Systems in Japan: First 200 Systems Results of Demonstration Research on Clustered PV Systems." In: Proceedings of the 20th European Photovoltaic Solar Energy Conference, June 6-10, Barcelona, Spain, pp. 2466-2469. WIP-Renewable Energies, Munich, Germany.

Ueda Y, T Oozeki, K Kurokawa, T Itou, K Kitamura, Y Miyamoto, M Yokota, and H Sugihara. 2005b. "Quantitative Analysis Method of Output Loss due to Restriction for Grid-Connected PV Systems." IEEJ Transactions on Power and Energy 125(12):1317-1326. 


\section{Mechanism I: Wind Energy Integration}

\section{I.1 Introduction}

This section discusses the role of a smart grid in helping to mitigate challenges for integrating wind energy into the electric system. The contribution of electricity generated by wind turbines is increasing due to a combination of the improved economic competitiveness of wind power, state and federal tax credits, state renewable energy portfolio requirements, and consumer desire to purchase "green" electricity. However, the integration of wind energy poses challenges due to the unpredictability and steep ramp rates of wind resources, which must be compensated by the use of more traditional power plants (termed load following or regulation) that increase costs because of redundancy and maintenance to correct increased wear and tear. Smart grid technologies, primarily communication and control over demand response and distributed generation and storage resources, can help replace fossil fuel capacity used to overcome the unpredictability and ramping issues, and thereby increase the level of wind generation into the electric system.

\section{I.2 Review}

Wind resources are characterized as being intermittent and having fast ramp rates (up and down), both of which increase the need for ancillary services such as regulation, load following, and reserves. The need for these services will increase in order to integrate increasing amounts of wind power penetrating the system. An illustration of the intermittency and ramping problem is illustrated by an event in February 2008, in Texas, in which the Electric Reliability Council of Texas (ERCOT) had to curtail power to interruptible customers when the grid frequency dropped rapidly because wind production suddenly fell 1700 megawatts.

The varying time scale for each of the three ancillary services is shown in Figure I.1. Regulation services must be performed all the time and require a fast communication link from the system operator to receive instruction to vary the generation set point on a second-by-second basis. Load following usually is required during significant load changes (ramp-up in the morning and ramp-down in the evening). This service requires changes in generation over several minutes to a few hours. Several organized power markets allow load customers to participate in selected ancillary services markets. For example, the PJM Interconnection in the United States offers participation in the competitive ancillary services markets (PJM 2009). Scheduling involves forecasting/anticipating loads in the next hour to days and assigning generation and transmission resources to meet these loads. 


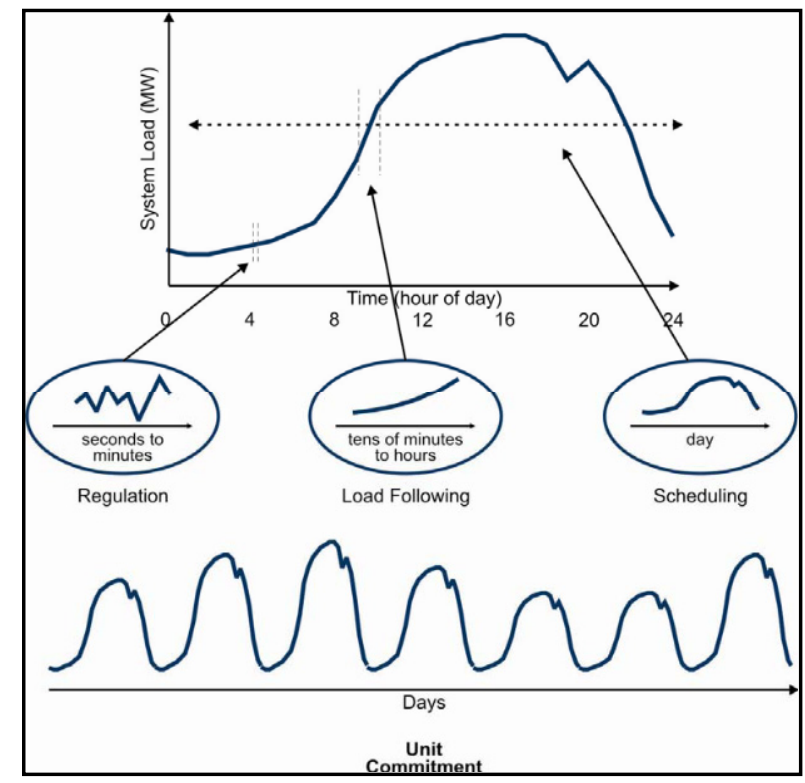

Source: Smith et al. 2007

Figure I.1. An Illustration of the Time Scale for Ancillary Services

Grid operators in the western United States estimated the additional ancillary service requirements to accommodate future large intermittent renewable resource integrations into the existing grid. The Bonneville Power Administration (BPA) performed a wind integration analysis in 2007 that indicated regulation requirements must be increased by about $50 \mathrm{MW}$, load following services by $210 \mathrm{MW}$, and contingency reserves by about $40 \mathrm{MW}$ to accommodate $3 \mathrm{GW}$ of additional wind capacity in the BPA footprint (NWPPC 2007). The California Independent System Operations (ISO), in a recent wind integration study, suggested that for a $20 \%$ renewable portfolio standard (RPS) to be realized, anywhere from 900 to $1500 \mathrm{MW}$ of 3-hour ramping capability needed to be contracted; regulation capacity requirements would increase by 170 to $250 \mathrm{MW}$ for ramp-up and 100 to $500 \mathrm{MW}$ for ramp-down services (CAISO 2007).

In 2007, the Federal Energy Regulatory Commission (FERC) directed the ISO/Regional Transmission Organizations (RTOs) to increase the transparency and openness of the transmission grid, facilitate access to new generating resources, and eliminate discriminatory practices that include the provision of ancillary services (FERC 2007). In 2008, the New York ISO initiated a market integration effort specifically designed for energy storage resources to provide regulation services. In 2008, the ISO New England changed its market rule (Market Rule 1) to remove the barriers that prevent non-generating resources from participating in regulation and frequency response services (CAISO 2009).

The Midwest ISO has proposed the creation of a new resource type, termed a stored energy resource, and proposed a set of operating parameters that are unique for this new resource. PJM Interconnect was the first U.S. RTO that offered load customers an option to participate in ancillary services, as early as 2006 (PJM 2006, 2009). Primarily large industrial load customers above a 1-MW threshold, such as aluminum smelters, showed interest in this market. Alcoa Inc. coordinated with the Midwest ISO to demonstrate, at its Warrick facility in southern Indiana, the provision of up to $15 \mathrm{MW}$ of regulation services, the role that demand response can play in regulation services (Todd et al. 2009). 
Implementation of regulation and load following services require telemetry and telecommunications to the grid operator. Ancillary service manuals published by the grid operator are very explicit in specifying performance standards, telecommunication requirements, and other eligibility requirements for the resource (load or generator). The eligibility requirement is usually a minimum resource size (in most cases $1 \mathrm{MW}$ ) to participate in the ancillary markets. The minimum resource size and the telemetry requirements are usually the major barriers for smaller distributed load resources to be utilized in these reserve and regulation markets. A smart grid is capable of delivering ancillary services on both counts. With respect to the minimum resource requirement, the smart grid can easily aggregate smaller units' demand response from many, rather than a single customer. The strengths of a smart grid are its real-time ability to respond coupled with the lower risk of meeting the minimum load requirement. Implementation requires recognition of these strengths through inclusion of these types of resources in ancillary service manuals.

In Minnesota, the RPS for 2025 is $25 \%$ from renewable energy. This amount (25\%) was studied for a four-utility combined balancing area that had a peak load of 20,984 MW. The estimated regulation requirement increased from $0.65 \%$ in the base case to $0.75 \%$ at the RPS level of $25 \%$. More telling, the total operating reserve (regulation, spinning, non-spinning, load following, and reserve margin) increased from $5 \%$ of the balancing area peak load capacity to $7 \%$ in the RPS $25 \%$ case (Smith et al. 2007).

A review of eight studies on wind integration on utilities (Parsons et al. 2006) showed that the cost of ancillary services to integrate wind ranged from approximately $\$ 2$ to $\$ 5 / \mathrm{MWh}$, with the cost generally increasing with the penetration from approximately $4 \%$ to $30 \%$. Cost increases associated with the provision of ancillary services needed to accommodate increasing penetration of wind can be moderated with the integration of large and diverse balancing areas, improved forecasting, and cooperative markets for ancillary services (Parsons et al. 2006). For wind penetration of up to 20\%, operating cost increases are less than or equal to $10 \%$ of the wholesale value of the wind energy. However, it is not known if the relationship is linear and can be scaled to higher wind penetration (Smith et al. 2007).

\section{I.3 Summary}

Wind energy has benefitted greatly from RPSs and tax credits, but it is characterized by intermittency and ramping that requires additional capacity to provide ancillary services in the form of regulation, load following, and scheduling. A review of efforts indicates that the electrical system can accommodate penetrations of wind energy on the order of $20 \%$ to $25 \%$ at little additional cost for the additional capacity needed to provide ancillary services. The studies also indicate that wind integration is facilitated in cases where the service area is geographically large and has a diversity of loads. The contribution of the smart grid technology can be to replace the additional capacity used to provide ancillary services with demand response by using advanced communication and control technologies, as evidenced by a limited demonstration.

The calculated energy and $\mathrm{CO}_{2}$ emissions for an RPS of $20 \%$ without smart grid technologies are presented in this paragraph. It is assumed that there are no end-use energy reductions, so the delivered $\mathrm{kWh}$ will be the same without and with RPS, but there is a reduction in input fossil energy associated with generating this electricity. An estimate of this is the average $\mathrm{kWh} / \mathrm{GW}$ capacity of approximately $4,300,000 \mathrm{kWh} / \mathrm{GW}$. Applying the average heat rate of approximately 10,000 Btu/kWh for conventional thermal generation, the reduction in primary energy is approximately 6.9 quads. This amounts to 
approximately $20 \%$ of the 34 quads of primary fossil energy consumed by the utility sector. In conjunction, there will be a decrease in $\mathrm{CO}_{2}$ emissions. At average $\mathrm{CO}_{2}$ emissions of $2.6 \mathrm{MMT} / \mathrm{GW}$, this translates to approximately $416 \mathrm{MMT}$ or approximately $14 \%$ of total utility $\mathrm{CO}_{2}$ emissions. A refinement to this first-order estimate would develop an estimate of the $\mathrm{CO}_{2}$ emission of the fossil-fueled capacity displaced, rather than the average for the electricity sector.

The additional reductions in electricity and $\mathrm{CO}_{2}$ emissions attributable to implementation of smart grid technologies that are calculated in Attachment 2 are based on the literature review and the author's experience. In the case of the direct reduction, it is estimated that an additional $0.1 \%$ of regulation is needed for peak load periods to meet the $20 \%$ RPS and that the energy and carbon reductions are $20 \%$ of this peak load, with a range of $10 \%$ to $30 \%$. The estimate of indirect reductions is based on the reduced need for the $2 \%$ increase in operating margin, with a range of $1 \%$ to $3 \%$. The reader is reminded that these are first-order estimates.

\section{I.4 Recommendation}

Work to date indicates that the grid can accommodate $20 \%$ to $25 \%$ penetration of wind power at minimal cost for the additional capacity needed to provide ancillary services, but the work to date leaves three questions unanswered:

- Is there an asymptotic limit to the penetration of wind? In other words, what is the limit that wind penetration is maximized $-100 \%$ of load with no ancillary services, $80 \%$ of load with $20 \%$ served by ancillary services, or something closer to an RPS of $25 \%$ ?

- Coupled with the above question, how is the asymptotic limit affected by geographic size and load diversity?

- An addition need is to quantify the contribution that storage and demand response enabled by smart grid technologies that can serve as a substitute for the fossil-fueled capacity used to provide ancillary services? The smart-grid-enabled regulation then offsets the need for reserve generating resources or curtailments, thus easing operational stresses and reducing the cost of managing high penetration of intermittent wind resources.

Additional work needs to provide answers to these three questions, and there is a need to examine the penetrations of wind and solar PV in conjunction with exploration of ranges in the size of geographic size, load diversity, storage, and demand response to provide guidelines for implementation efforts by utilities.

\section{I.5 References}

California Independent System Operator Corporation (CAISO). 2007. Integration of Renewable Resources; Transmission and Operating Issues and Recommendations for Integrating Renewable Resources on the California ISO-Controlled Grid. California Independent System Operator Corporation, Folsom, California.

California Independent System Operator Corporation (CAISO). 2009. Participation of Limited Energy Storage Resources in CAISO Electricity Markets. California Independent System Operator Corporation, Folsom, California. 
Federal Energy Regulatory Commission (FERC). 2007. "Preventing Undue Discrimination and Preference in Transmission Service." Order No. 890, Federal Energy Regulatory Commission, Washington, D.C.

Northwest Power and Conservation Council (NWPPC). 2007. The Northwest Wind Integration Action Plan. WIF Document 2007-1, Portland, Oregon.

Parsons B, M Milligan, JC Smith, K Porter, E Demeo, B Oakleaf, K Wolf, R Zavadil, H Shiu, and DY Nakafuji. 2006. "Grid Impacts of Wind Power Variability: Recent Assessments from a Variety of Utilities in the United States." In: European Wind Energy Conference, February 27-March 2, Athens, Greece. NREL/CP-500-39955.

PJM Interconnection. 2006a. "PJM Ancillary Services Markets Opened to Demand Response." Press Release, PJM Interconnection, Norristown, Pennsylvania.

PJM Interconnection. 2006b. A Clear Focus. PJM Annual Report. PJM Interconnection, Norristown, Pennsylvania.

PJM Interconnection. 2009. Manual 11: Scheduling Operations. Revision: 38. PJM Interconnection, Norristown, Pennsylvania.

Smith JC, B Parsons, T Acker, M Milligan, R Zavadil, M Schuerger, and E DeMeo. 2007. "Best Practices in Grid Integration of Variable Wind Power: Summary of Recent U.S. Case Study Results and Mitigation Measures." In: European Wind Energy Conference 2007, May, Milan, Italy. Accessed August 5, 2009, at http://www.uwig.org/opimpactsdocs.html.

Todd D, M Caufield, B Helms, M Starke, B Kirby, and J Kueck. 2009. Providing Reliability Services through Demand Response: A Preliminary Evaluation of the Demand Response Capabilities of Alcoa Inc. ORNL/TM-2008/233, Oak Ridge National Laboratory, Oak Ridge, Tennessee. 

Attachment 2

Electricity and $\mathrm{CO}_{2}$ Reduction Calculations 



\section{Attachment 2 - Electricity and $\mathrm{CO}_{2}$ Reduction Calculations}

This attachment provides the methodology used to calculate the reduction in electricity use and $\mathrm{CO}_{2}$ emissions and the results of those calculations for each of the nine mechanisms. The measures are separated into those with reductions that result directly with the application of the smart grid technologies, and those for which reductions are obtained indirectly through reinvestment of avoided capital investment due to smart grid technologies. The calculations are based in the year 2030 for both direct and indirect reductions.

The improvement in efficiency and/or capacity associated with the mechanisms is drawn from the respective mechanism discussion in Attachment 1. The energy, electricity, capacity, and $\mathrm{CO}_{2}$ emissions data is drawn from EIA Annual Energy Outlook (AEO) for 2008, supplemented in some cases by the latest issue of the Commercial Building Energy Consumption Survey (CBECS), and Residential Energy Consumption Survey (RECS).

\section{Direct Reduction Methodology}

The direct reductions are basically the product of the efficiency improvement for the respective mechanism from Attachment 1 and the electricity consumption for the sector being examined.

The calculation for $\mathrm{CO}_{2}$ emissions is based on the share of electricity reduced by the smart grid technologies associated with the mechanism to total electricity delivered to the grid, hence the $\mathrm{CO}_{2}$ reduction estimate is generally the same as the electricity reduction estimate. In cases where the $\mathrm{CO}_{2}$ reductions differ from the electricity reductions, it is expected that the mechanism affects one type of generation over another.

Direct mechanisms include:

- Conservation Effect of Consumer Information and Feedback Systems

- Deployment of Diagnostics in Residential and Small/

- Small/Medium Commercial Buildings

- Measurement \& Verification (M\&V) for Energy Efficiency Programs

- Shifting Load to More Efficient Generation

- Support Additional Electric Vehicles and Plug-In Hybrid Electric Vehicles

- Conservation Voltage Reduction and Advanced Voltage Control

- Support Penetration of Renewable Solar Generation (25\% renewable portfolio standard [RPS]).

- Support Penetration of Renewable Wind Generation (25\% RPS).

\section{Indirect Reduction Methodology}

The indirect reductions are calculated as the cost of capacity that is not built, or the cost of program activities not incurred as a result of the implementing the smart grid technologies associated with the mechanism. The monetary value of the avoided cost is then assumed to be reinvested in efficiency, with 
the quantity of efficiency expressed as $\mathrm{kWh}$ and valued at the average price of electricity. In the case of capital displacements, the calculations do not include other fixed and variable costs that may be associated.

Note that the additional reductions may not be realized if reinvestment does not occur.

The calculation for $\mathrm{CO}_{2}$ emissions is based on the share of electricity reduced by the smart grid technologies associated with the mechanism to total electricity delivered to the grid.

Indirect mechanisms documented include:

- Joint Marketing of Energy Efficiency and Demand Response Programs

- M\&V for Energy Efficiency Programs

- Support Penetration of Renewable Solar Generation (25\% RPS).

- Support Penetration of Renewable Wind Generation (25\% RPS).

\section{Calculations}

The calculation for the direct and indirect reductions for each of the mechanisms follows in this attachment. The basic assumptions and results are summarized in a table, with the calculation details, assumptions, and data sources provided below the table.

The uncertainty surrounding the estimated electricity and $\mathrm{CO}_{2}$ emission were not calculated because the estimation methodology is not tailored to provide specific estimates for each of the mechanisms. The estimated reduction in the tables is developed from estimated reduction in the first column. The low and high reduction levels provided in columns two and three provide an estimate of the uncertainty with respect to the estimated reduction and can be used to scale the estimate provided. In addition, the estimates provided assume $100 \%$ penetration of the smart grid technologies within the respective sectors. This enables the estimates to be reduced in proportion to the fraction of U.S. electricity consumers served by utilities deploying a smart grid, as a first-order approximation. 


\section{Direct Savings Mechanism A \\ Conservation Effect of Consumer Information and Feedback Systems}

\begin{tabular}{|c|c|c|c|c|c|c|c|c|}
\hline \multirow[b]{3}{*}{$\begin{array}{c}\text { Est. } \\
\%\end{array}$} & \multicolumn{4}{|c|}{ Reduced Energy Consumption (2030) } & \multicolumn{4}{|c|}{ Electric Sector Annual Reductions (2030) } \\
\hline & \multirow[b]{2}{*}{$\begin{array}{c}\text { Low } \\
\%\end{array}$} & \multirow[b]{2}{*}{$\begin{array}{c}\text { High } \\
\%\end{array}$} & \multicolumn{2}{|c|}{ Baseline Electricity Consumption } & \multicolumn{2}{|c|}{ Energy } & \multicolumn{2}{|c|}{ Carbon Emissions } \\
\hline & & & End-Use Sector(s) & $\left(10^{9} \mathrm{kWh} /\right.$ year $)$ & $\begin{array}{l}\% \text { of } \\
\text { United } \\
\text { States }\end{array}$ & $\begin{array}{c}\left(10^{9} \mathrm{kWh} /\right. \\
\text { year })\end{array}$ & $\begin{array}{l}\% \text { of } \\
\text { United } \\
\text { States }\end{array}$ & $\begin{array}{c}\text { (MMT/ } \\
\text { yearr) }\end{array}$ \\
\hline 6 & 1 & 10 & Residential & 1722 & 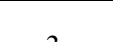 & $1--$ & ( & 0 \\
\hline 6 & 1 & 10 & Small/Medium Commercial Buildings & 854 & 3 & 155 & 3 & 92 \\
\hline
\end{tabular}

- Residential Sector

- The quantity of electricity reduction is calculated by applying the reduction (6\%) in electricity consumption to the quantity of electricity consumed by the sector (1722 B kWh - AEO), which equals approximately $103 \mathrm{~B} \mathrm{kWh}$.

- The percentage reduction in utility sector electricity (approximately $2 \%$ ) is the sector reduction (103 B kWh) divided by total utility sector electricity delivered to the grid (4968 B kWh -AEO).

- The quantity of $\mathrm{CO}_{2}$ emissions reduced (61 MMT) is calculated by applying the percentage reduction in utility sector electricity consumption (approximately $2 \%$ ) to electric utility sector $\mathrm{CO}_{2}$ emissions (2948 MMT - AEO).

- The percentage reduction in $\mathrm{CO}_{2}$ emissions (approximately $2 \%$ ) is calculated by dividing the quantity of reduced $\mathrm{CO}_{2}$ emissions (61 MMT) by utility sector emissions (2948 MMT -AEO).

- Small/Medium Commercial Buildings

- The quantity of electricity reduction is calculated by applying the reduction (6\%) in electricity consumption to the quantity of electricity consumed by the Small/Medium Commercial building sector (854 B kWh), which equals approximately $51 \mathrm{~B} \mathrm{kWh/year} \mathrm{Small/Medium} \mathrm{Commercial}$ building consumption ( $854 \mathrm{~B} \mathrm{kWh}$ ) is calculated by multiplying the $2003 \mathrm{Small} /$ Medium Commercial building share of electricity (44\% - CBECS) by commercial sector consumption (1941 B kWh - AEO).

- The percentage reduction in utility sector electricity (approximately $1 \%$ ) is the sector reduction (51 B kWh) divided by total utility sector electricity delivered to the grid (4968 B kWh -AEO).

- The quantity of $\mathrm{CO}_{2}$ emissions reduced (30 MMT) is calculated by applying the percentage reduction in utility sector electricity consumption (approximately 1\%) to electric utility sector $\mathrm{CO}_{2}$ emissions (2948 MMT - AEO).

- The percentage reduction in $\mathrm{CO}_{2}$ emissions (approximately $1 \%$ ) is calculated by dividing the quantity of reduced $\mathrm{CO}_{2}$ emissions (30 MMT) by utility sector emissions (2948 MMT -AEO).

Assumptions

- Sector savings of $6 \%$ from range of $1 \%$ to $10 \%$

- Penetration $=100 \%$

- Small/Medium Commercial buildings are defined as less than 50,000 $\mathrm{ft}^{2}$ in floor area. 
- The 2030 Small/Medium Commercial building share of total commercial sector electricity consumption is the same as the 2003 share.

\section{Data Sources}

- Residential Sector electricity consumption in 2030 is $1722 \mathrm{~B} \mathrm{kWh}$ Source: AEO 2008 (DOE/EIA-0383), Table 8. Electricity Supply, Disposition, Prices, and Emissions; Electricity Sales by Sector

- Commercial Sector electricity consumption in 2030 is $1941 \mathrm{~B} \mathrm{kWh}$ Source: AEO 2008 (DOE/EIA-0383), Table 8. Electricity Supply, Disposition, Prices, and Emissions; Electricity Sales by Sector

- Small/Medium Commercial building consumption as share of total commercial sector consumption in 2003 is $44 \%$

Source: 2003 CBECS Detailed Tables, Table E5A. Electricity Consumption (kWh) by End Use for All Buildings, 2003

- Total Electricity Supply to the grid in 2030 is $4968 \mathrm{~B} \mathrm{kWh} /$ year Source: AEO 2008 (DOE/EIA-0383), Table 8. Electricity Supply, Disposition, Prices, and Emissions; Total Net Generation to the Grid

- Electric Utility $\mathrm{CO}_{2}$ Emissions in 2030 is $2948 \mathrm{MMT}$

Source: AEO 2008 (DOE/EIA-0383), Table 18. Carbon Dioxide Emissions by Sector and Source; Electric Power Total 


\section{Direct Savings Mechanism C \\ Deployment of Diagnostics in Residential and Small/Medium Commercial Buildings}

\begin{tabular}{|c|c|c|c|c|c|c|c|c|}
\hline \multirow[b]{3}{*}{$\begin{array}{c}\text { Est. } \\
\%\end{array}$} & \multicolumn{4}{|c|}{ Reduced Energy Consumption (2030) } & \multicolumn{4}{|c|}{ Electric Sector Annual Reductions (2030) } \\
\hline & \multirow[b]{2}{*}{$\begin{array}{c}\text { Low } \\
\%\end{array}$} & \multirow[b]{2}{*}{$\begin{array}{l}\text { High } \\
\%\end{array}$} & \multicolumn{2}{|c|}{ Baseline Electricity Consumption } & \multicolumn{2}{|c|}{ Energy } & \multicolumn{2}{|c|}{ Carbon Emissions } \\
\hline & & & End-Use Sector(s) & $\left(10^{9} \mathrm{kWh} /\right.$ year $)$ & $\begin{array}{c}\text { \% of } \\
\text { United } \\
\text { States }\end{array}$ & $\begin{array}{c}\left(10^{9} \mathrm{kWh} /\right. \\
\text { year })\end{array}$ & $\begin{array}{c}\% \text { of } \\
\text { United } \\
\text { States }\end{array}$ & $\begin{array}{c}\text { (MMT/ } \\
\text { yearr) }\end{array}$ \\
\hline 15 & 10 & 20 & $\begin{array}{l}\text { Residential (Heat Pump \& Air } \\
\text { Conditioner) }\end{array}$ & 331 & \multirow{2}{*}{3} & \multirow{2}{*}{152} & \multirow{2}{*}{3} & \multirow{2}{*}{90} \\
\hline 20 & 10 & 30 & $\begin{array}{l}\text { Small/Medium Commercial } \\
\text { Buildings (HVAC + Lighting) }\end{array}$ & 510 & & & & \\
\hline
\end{tabular}

- Residential Sector

- The quantity of electricity reduction is calculated by applying the reduction (15\%) in electricity consumption to the quantity of electricity consumed by heat pumps and air conditioners $(331 \mathrm{~B}$ $\mathrm{kWh}$ ), which equals approximately $50 \mathrm{~B} \mathrm{kWh}$. Heat pump energy is the product of the share of electricity consumed by heat pumps in 2005 (27\% - 2005 RECS) and the delivered heating energy 2030 (0.33 quad - AEO). Delivered energy consumed for air-conditioning is 1.04 quads (AEO). The sum of heat pump and air-conditioning delivered energy is then converted to $\mathrm{kWh}$ by dividing by $3412 \mathrm{Btu} / \mathrm{kWh}$ for $331 \mathrm{~B} \mathrm{kWh}$.

- The percentage reduction in utility sector electricity (approximately $1 \%$ ) is the sector reduction (50 B kWh) divided by total utility sector electricity delivered to the grid (4968 B kWh -AEO).

- The quantity of $\mathrm{CO}_{2}$ emissions reduced (29 MMT) is calculated by applying the percentage reduction in utility sector electricity consumption (approximately $1 \%$ ) to electric utility sector $\mathrm{CO}_{2}$ emissions (2948 MMT - AEO).

- The percentage reduction in $\mathrm{CO}_{2}$ emissions (approximately $1 \%$ ) is calculated by dividing the quantity of reduced $\mathrm{CO}_{2}$ emissions (29 MMT) by utility sector emissions (2948 MMT -AEO).

- Small/Medium Commercial Buildings

- The quantity of electricity reduction is calculated by applying the reduction (20\%) in electricity consumption to the quantity of energy consumed by Small/Medium Commercial buildings for heating, ventilating, and air conditioning (HVAC) and lighting (510 B kWh), which equals approximately $102 \mathrm{~B} \mathrm{kWh}$ /year. Small/Medium Commercial building HVAC and lighting consumption (510 B kWh) is calculated by multiplying the 2003 Small/Medium Commercial building share of HVAC and lighting (20\% - CBECS) by commercial sector consumption in 2030 (1941 B kWh).

- The percentage reduction in utility sector electricity (approximately $2 \%$ ) is the sector reduction (102 B kWh) divided by total utility sector electricity delivered to the grid (4968 B kWh -AEO).

- The quantity of $\mathrm{CO}_{2}$ emissions reduced (61 MMT) is calculated by applying the percentage reduction in utility sector electricity consumption (approximately $2 \%$ ) to electric utility sector $\mathrm{CO}_{2}$ emissions (2948 MMT - AEO). 
- The percentage of emission reductions (approximately $2 \%$ ) were calculated by dividing the quantity of energy reductions (102 B kWh) by the total electricity supplied to the grid (4968 B $\mathrm{kWh}$ - AEO) times total utility sector emissions (2948 MMT -AEO).

\section{$\underline{\text { Assumptions }}$}

- Residential sector savings of $15 \%$ from range of $10 \%$ to $20 \%$ and commercial sector savings of $20 \%$ from range of $10 \%$ to $30 \%$.

- Penetration $=100 \%$

- The share of residential sector electricity heating consumed by heat pumps in 2030 is the same as in 2005 .

- Small/Medium Commercial buildings are defined as less than $50,000 \mathrm{ft}^{2}$ in floor area.

- The 2030 Small/Medium Commercial building share of total commercial sector electricity consumption for HVAC is the same as the 2003 share.

\section{Data Sources}

- 2005 Share of residential electricity consumed by heat pumps is $27 \%$. Source: RECS 2005, Table HC2.4. Space Heating Characteristics by Type of Housing Unit, 2005.

- Residential Sector electricity consumption in 2030 for heating is 0.33 quad and air conditioning is 1.04 quads.

Source: AEO 2008 (DOE/EIA-0383), Table 4. Residential Sector Key Indicators and Consumption

- Commercial Sector electricity consumption is $1941 \mathrm{~B} \mathrm{kWh}$ in 2030

Source: AEO 2008 (DOE/EIA-0383), Table 8. Electricity Supply, Disposition, Prices, and Emissions; Electricity Sales by Sector

- Small/Medium Commercial building HVAC consumption as share of total commercial sector consumption in 2003 is $26 \%$

Source: 2003 CBECS Detailed Tables, Table E5A. Electricity Consumption (kWh) by End Use for All Buildings, 2003

- Electricity Supply in 2030 is $4968 \mathrm{~B} \mathrm{kWh}$

Source: AEO 2008 (DOE/EIA-0383), Table 8. Electricity Supply, Disposition, Prices, and Emissions; Total Net Generation to the Grid

- Electric Utility $\mathrm{CO}_{2}$ Emissions in 2030 is $2948 \mathrm{MMT}$

Source: AEO 2008 (DOE/EIA-0383), Table 18. Carbon Dioxide Emissions by Sector and Source; Electric Power Total 


\section{Direct Savings Mechanism D Measurement \& Verification for Energy Efficiency Programs}

\begin{tabular}{|c|c|c|c|c|c|c|c|c|}
\hline \multirow[b]{3}{*}{$\begin{array}{c}\text { Est. } \\
\%\end{array}$} & \multicolumn{4}{|c|}{ Reduced Energy Consumption (2030) } & \multicolumn{4}{|c|}{ Electric Sector Annual Reductions (2030) } \\
\hline & \multirow[b]{2}{*}{$\begin{array}{c}\text { Low } \\
\%\end{array}$} & \multirow[b]{2}{*}{$\underset{\%}{\mathrm{High}}$} & \multicolumn{2}{|c|}{ Baseline Electricity Consumption } & \multicolumn{2}{|c|}{ Energy } & \multicolumn{2}{|c|}{ Carbon Emissions } \\
\hline & & & End-Use Sector(s) & $\left(10^{9} \mathrm{kWh} /\right.$ year $)$ & $\begin{array}{c}\% \text { of } \\
\text { United } \\
\text { States }\end{array}$ & $\begin{array}{c}\left(10^{9} \mathrm{kWh} /\right. \\
\text { year })\end{array}$ & $\begin{array}{c}\% \text { of } \\
\text { United } \\
\text { States }\end{array}$ & $\begin{array}{c}\text { (MMT/ } \\
\text { yearr) }\end{array}$ \\
\hline 7 & 5 & 20 & $\begin{array}{l}\text { Residential (Heat Pump \& Air } \\
\text { Conditioner) }\end{array}$ & 331 & \multirow{2}{*}{1} & 59 & \multirow{2}{*}{1} & \multirow{2}{*}{35} \\
\hline 7 & 5 & 20 & $\begin{array}{l}\text { Small/Medium Commercial } \\
\text { Buildings (HVAC + Lighting) }\end{array}$ & 510 & & 155 & & \\
\hline
\end{tabular}

- Residential Sector

- The quantity of electricity reduction is calculated by applying the reduction (7\%) in electricity consumption to the quantity of electricity consumed by heat pumps and air conditioners $(331 \mathrm{~B}$ $\mathrm{kWh}$ ), which equals approximately $23 \mathrm{~B} \mathrm{kWh}$. Heat pump energy is the product of the share of electricity consumed by heat pumps in 2005 (27\% - 2005 RECS) and the delivered heating energy 2030 (0.33 quad - AEO). Energy consumed for air conditioning is 1.04 quads (AEO). The sum of heat pump and air-conditioning-delivered energy is then converted to $\mathrm{kWh}$ by dividing by $3412 \mathrm{Btu} / \mathrm{kWh}$ for $331 \mathrm{~B} \mathrm{kWh}$.

- The percentage reduction in utility sector electricity (approximately $0.5 \%$ ) is the sector reduction (23 B kWh) divided by total utility sector electricity delivered to the grid (4968 B kWh -AEO).

- The quantity of $\mathrm{CO}_{2}$ emissions reduced (14 MMT) is calculated by applying the percentage reduction in utility sector electricity consumption (approximately $0.5 \%$ ) to electric utility sector $\mathrm{CO}_{2}$ emissions (2948 MMT - AEO).

- The percentage reduction in $\mathrm{CO}_{2}$ emissions (approximately $0.5 \%$ ) is calculated by dividing the quantity of reduced $\mathrm{CO}_{2}$ emissions (14 MMT) by utility sector emissions (2948 MMT -AEO).

- Small/Medium Commercial Buildings

- The quantity of electricity reduction is calculated by applying the reduction (7\%) in electricity consumption to the quantity of energy consumed by Small/Medium Commercial buildings for HVAC, and lighting (510 B kWh), which equals approximately $36 \mathrm{~B} \mathrm{kWh/year.} \mathrm{Small/Medium}$ Commercial building HVAC and lighting consumption (510 B $\mathrm{kWh}$ ) is calculated by multiplying the 2003 Small/Medium Commercial building share of HVAC and lighting (20\% - CBECS) by commercial sector consumption in 2030 (1941 B kWh).

- The percentage reduction in utility sector electricity (approximately $0.7 \%$ ) is the sector reduction (36 B kWh) divided by total utility sector electricity delivered to the grid (4968 B kWh -AEO).

- The quantity of $\mathrm{CO}_{2}$ emissions reduced (21 MMT) is calculated by applying the percentage reduction in utility sector electricity consumption (approximately $0.7 \%$ ) to electric utility sector $\mathrm{CO}_{2}$ emissions (2948 MMT - AEO).

- The percentage emission reductions (approximately $0.7 \%$ ) were calculated by dividing the quantity of energy reductions (36 B kWh) by the total electricity supplied to the grid (4968 B $\mathrm{kWh}$ - AEO) times total utility sector emissions (2948 MMT -AEO). 


\section{Assumptions}

- Sector savings of $7 \%$ from range of $5 \%$ to $20 \%$

- Penetration $=100 \%$

- The share of residential sector electricity heating consumed by heat pumps in 2030 is the same as in 2005.

- Small/Medium Commercial buildings are defined as less than 50,000 $\mathrm{ft}^{2}$ in floor area.

- The 2030 Small/Medium Commercial building share of total commercial sector electricity consumption for HVAC is the same as the 2003 share.

\section{$\underline{\text { Data Sources }}$}

- 2005 Share of residential electricity consumed by heat pumps is $27 \%$. Source: RECS 2005, Table HC2.4, Space Heating Characteristics by Type of Housing Unit, 2005.

- Residential Sector electricity consumption in 2030 for heating is 0.33 quad and air conditioning is 1.04 quads.

Sources: AEO 2008 (DOE/EIA-0383), Table 4. Residential Sector Key Indicators and Consumption

- Small/Medium Commercial building HVAC electricity consumption as share of total commercial sector consumption in 2003 is $26 \%$

Source: 2003 CBECS Detailed Tables, Table E5A. Electricity Consumption (kWh) by End Use for All Buildings, 2003

- Commercial Sector electricity consumption in 2030 is $1941 \mathrm{~B} \mathrm{kWh}$

Source: AEO 2008 (DOE/EIA-0383), Table 8. Electricity Supply, Disposition, Prices, and Emissions; Electricity Sales by Sector

- Electricity Supply in 2030 is $4968 \mathrm{~B} \mathrm{kWh}$

Source: AEO 2008 (DOE/EIA-0383), Table 8. Electricity Supply, Disposition, Prices, and Emissions; Total Net Generation to the Grid

- Electric Utility $\mathrm{CO}_{2}$ Emissions in 2030 is 2948 MMT

Source: AEO 2008 (DOE/EIA-0383), Table 18. Carbon Dioxide Emissions by Sector and Source; Electric Power Total 


\section{Direct Savings Mechanism E Shifting Load to More Efficient Generation}

\begin{tabular}{|c|c|c|c|c|c|c|c|c|}
\hline \multicolumn{5}{|c|}{ Reduced Energy Consumption (2030) } & \multicolumn{4}{|c|}{ Electric Sector Annual Reductions (2030) } \\
\hline \multirow[b]{2}{*}{$\begin{array}{c}\text { Est. } \\
\%\end{array}$} & \multirow[b]{2}{*}{$\begin{array}{c}\text { Low } \\
\%\end{array}$} & \multirow[b]{2}{*}{$\begin{array}{c}\text { High } \\
\%\end{array}$} & \multicolumn{2}{|c|}{ Baseline Electricity Consumption } & \multicolumn{2}{|c|}{ Energy } & \multicolumn{2}{|c|}{ Carbon Emissions } \\
\hline & & & End-Use Sector(s) & $\left(10^{9} \mathrm{kWh} /\right.$ year $)$ & $\begin{array}{c}\% \text { of } \\
\text { United } \\
\text { States }\end{array}$ & $\begin{array}{c}\left(10^{9} \mathrm{kWh} /\right. \\
\text { year })\end{array}$ & $\begin{array}{c}\% \text { of } \\
\text { United } \\
\text { States }\end{array}$ & $\begin{array}{c}\text { (MMT/ } \\
\text { yearr) }\end{array}$ \\
\hline 0.04 & 0.02 & 0.06 & Total Electric Supply & 4968 & 0.04 & 2 & 0.03 & 1 \\
\hline
\end{tabular}

\section{- Total Electricity Supply}

- The quantity of electricity reduction $(2 \mathrm{~B} \mathrm{kWh})$ is calculated by applying the reduction $(0.04 \%)$ in electricity consumption to the quantity of electricity supplied to the grid (4968 B kWh - AEO).

- The percentage reduction $(0.04 \%)$ is calculated by dividing the quantity reduced $(2 \mathrm{~B} \mathrm{kWh})$ by the total quantity of electricity supplied to the grid (4968 B kWh - AEO).

- The $\mathrm{CO}_{2}$ emission reduction (1 MMT) is calculated as the product of emission reduction $(0.03 \%)$ and total utility sector emissions (2948 MMT -AEO).

- The percentage of emission reduction (approximately $0.03 \%$ ) is calculated as $75 \%$ of the percentage reduction in utility sector electricity consumption (approximately $0.04 \%$ ).

- The quantity of $\mathrm{CO}_{2}$ emissions reduced (1 MMT) is calculated as the product of the percentage reduction in emissions $(0.03 \%)$ and total utility emissions (2948 MMT).

\section{Assumptions}

- Load shifting reduction of $0.04 \%$ from range of $0.02 \%$ to $0.06 \%$.

- Penetration $=100 \%$

- The percentage reduction in $\mathrm{CO}_{2}$ emissions is approximately $75 \%$ of the electricity reduction.

\section{$\underline{\text { Data Sources }}$}

- Electricity Supply in 2030 is $4968 \mathrm{~B} \mathrm{kWh}$

Source: AEO 2008 (DOE/EIA-0383), Table 8. Electricity Supply, Disposition, Prices, and Emissions; Total Net Generation to the Grid

- Electric Utility $\mathrm{CO}_{2}$ Emissions in 2030 is 2948 MMT

Source: AEO 2008 (DOE/EIA-0383), Table 18. Carbon Dioxide Emissions by Sector and Source; Electric Power Total 


\section{Direct Savings Mechanism F Support Additional Electric Vehicles and Plug-In Hybrid Electric Vehicles}

\begin{tabular}{|c|c|c|c|c|c|c|c|c|}
\hline \multirow[b]{3}{*}{$\begin{array}{c}\text { Est. } \\
\%\end{array}$} & \multicolumn{4}{|c|}{ Reduced Energy Consumption (2030) } & \multicolumn{4}{|c|}{ Electric Sector Annual Reductions (2030) } \\
\hline & \multirow[b]{2}{*}{$\begin{array}{c}\text { Low } \\
\%\end{array}$} & \multirow[b]{2}{*}{$\begin{array}{c}\text { High } \\
\%\end{array}$} & \multicolumn{2}{|c|}{ Baseline Electricity Consumption } & \multicolumn{2}{|c|}{ Energy } & \multicolumn{2}{|c|}{ Carbon Emissions } \\
\hline & & & End-Use Sector(s) & $\left(10^{9} \mathrm{kWh} /\right.$ year $)$ & $\begin{array}{l}\% \text { of } \\
\text { United } \\
\text { States }\end{array}$ & $\begin{array}{c}\left(10^{9} \mathrm{kWh} /\right. \\
\text { year })\end{array}$ & $\begin{array}{l}\% \text { of } \\
\text { United } \\
\text { States }\end{array}$ & $\begin{array}{c}\text { (MMT/ } \\
\text { yearr) }\end{array}$ \\
\hline 3 & 2 & 5 & $\begin{array}{l}\text { Electricity Equivalent of Light } \\
\text { Vehicle Transportation (cars, vans, } \\
\text { SUVs, light trucks) }\end{array}$ & 5135 & 3 & 139 & 3 & 82 \\
\hline
\end{tabular}

Note: the estimated reductions are "well-to-wheel" and are expressed in $\mathrm{kWh}$. While electric generation increases to accommodate the additional vehicles, the petroleum energy that the vehicles would otherwise consume decreases.

- Transportation Sector, LDVs

- The quantity of electricity reduction (139 B kWh) is calculated by applying the reduction (3\%) in energy consumption to the quantity of total quantity of equivalent electricity consumed by LDVs $(5135 \mathrm{~B} \mathrm{kWh})$. This quantity is derived from the Btu quantity of energy consumed by LDVs (17.52 quads) to electricity consumption using a conversion factor of $3412 \mathrm{Btu} / \mathrm{kWh}$.

- The percentage reduction in electricity (3\%) is calculated by dividing the quantity reduced (139 B $\mathrm{kWh}$ ) by the total quantity of electricity supplied to the grid (4968 B kWh - AEO).

- The $\mathrm{CO}_{2}$ emission reduction (78 MMT) is calculated by dividing the quantity electricity reduction (131 B kWh) by the total electricity supplied to the grid (4968 B kWh - AEO) times total utility sector emissions (2948 MMT - AEO).

- The percentage of $\mathrm{CO}_{2}$ emission reduction (3\%) is calculated by dividing the reduction (78 MMT) by total utility emissions (2948 MMT -AEO).

\section{Assumptions}

- $\mathrm{PHEV} / \mathrm{EV}$ savings of $3 \%$, calculated as the product of a $9 \%$ increase in the vehicle fleet times a $30 \%$ increase in energy consumption per VMT, from a range of $2 \%$ to $5 \%$.

- Penetration $=100 \%$

\section{$\underline{\text { Data Sources }}$}

- LDV consumption in 2030 is 17.52 quads

Source: AEO 2008 (DOE/EIA-0383), Table 7. Transportation Sector Key Indicators and Delivered Energy Consumption

- Electricity Supply in 2030 is $4968 \mathrm{~B} \mathrm{kWh}$

Source: AEO 2008 (DOE/EIA-0383), Table 8. Electricity Supply, Disposition, Prices, and Emissions; Total Net Generation to the Grid

- Electric Utility $\mathrm{CO}_{2}$ Emissions in 2030 is $2948 \mathrm{MMT}$

Source: AEO 2008 (DOE/EIA-0383), Table 18. Carbon Dioxide Emissions by Sector and Source; Electric Power Total 


\section{Direct Savings Mechanism G Conservation Voltage Reduction and Advanced Volt/VAR Control}

\begin{tabular}{|c|c|c|c|c|c|c|c|c|}
\hline \multicolumn{5}{|c|}{ Reduced Energy Consumption (2030) } & \multicolumn{4}{|c|}{ Electric Sector Annual Reductions (2030) } \\
\hline \multirow[b]{2}{*}{$\begin{array}{c}\text { Est. } \\
\%\end{array}$} & \multirow[b]{2}{*}{$\begin{array}{c}\text { Low } \\
\%\end{array}$} & \multirow[b]{2}{*}{$\begin{array}{c}\text { High } \\
\%\end{array}$} & \multicolumn{2}{|c|}{ Baseline Electricity Consumption } & \multicolumn{2}{|c|}{ Energy } & \multicolumn{2}{|c|}{ Carbon Emissions } \\
\hline & & & End-Use Sector(s) & $\left(10^{9} \mathrm{kWh} /\right.$ year $)$ & $\begin{array}{c}\% \text { of } \\
\text { United } \\
\text { States }\end{array}$ & $\begin{array}{c}\left(10^{9} \mathrm{kWh} /\right. \\
\text { year })\end{array}$ & $\begin{array}{c}\% \text { of } \\
\text { United } \\
\text { States }\end{array}$ & $\begin{array}{c}\text { (MMT/ } \\
\text { yearr) }\end{array}$ \\
\hline 2 & 1 & 4 & Total Electric Supply & 4968 & 2 & 99 & 2 & 59 \\
\hline
\end{tabular}

- Distribution Sector

- The quantity of electricity reduction $(99 \mathrm{~B} \mathrm{kWh})$ is calculated by applying the reduction $(2 \%)$ in electricity consumption to the quantity of electricity supplied to the grid (4968 B kWh - AEO).

- The percentage reduction ( $2 \%$ ) is calculated by dividing the quantity reduced ( $99 \mathrm{~B} \mathrm{kWh}$ ) by the total quantity of electricity supplied to the grid (4968 B kWh - AEO).

- The $\mathrm{CO}_{2}$ emission reduction (59 MMT) is calculated by dividing the quantity electricity reduction (99 B kWh) by the total electricity supplied to the grid (4968 B kWh - AEO) times total utility sector emissions (2948 MMT -AEO).

- The percentage of $\mathrm{CO}_{2}$ emission reduction (2\%) is calculated by dividing the reduction (59 MMT) by total utility emissions (2948 MMT -AEO).

\section{Assumptions}

- Distribution savings of $2 \%$, corresponding to an average voltage reduction of 2.5 volts, from range of $1 \%$ to $4 \%$.

- Penetration $=100 \%$

\section{$\underline{\text { Data Sources }}$}

- Electricity Supply to the grid in 2030 is $4968 \mathrm{~B} \mathrm{kWh}$

Source: AEO 2008 (DOE/EIA-0383), Table 8. Electricity Supply, Disposition, Prices, and Emissions; Total Net Generation to the Grid

- Electric Utility $\mathrm{CO}_{2}$ Emissions in 2030 is 2948 MMT

Source: AEO 2008 (DOE/EIA-0383), Table 18. Carbon Dioxide Emissions by Sector and Source; Electric Power Total 


\section{Direct Savings Mechanism I \\ Support Penetration of Renewable Wind Generation (25\% RPS)}

\begin{tabular}{|c|c|c|c|c|c|c|c|c|}
\hline \multicolumn{5}{|c|}{ Reduced Energy Consumption (2030) } & \multicolumn{4}{|c|}{ Electric Sector Annual Reductions (2030) } \\
\hline \multirow[b]{2}{*}{$\begin{array}{c}\text { Est. } \\
\%\end{array}$} & \multirow[b]{2}{*}{$\begin{array}{l}\text { Low } \\
\%\end{array}$} & \multirow[b]{2}{*}{$\begin{array}{c}\text { High } \\
\% \\
\end{array}$} & \multicolumn{2}{|c|}{ Baseline Electricity Consumption } & \multicolumn{2}{|c|}{ Energy } & \multicolumn{2}{|c|}{ Carbon Emissions } \\
\hline & & & End-Use Sector(s) & $\left(10^{9} \mathrm{kWh} /\right.$ year $)$ & $\begin{array}{c}\% \text { of } \\
\text { United } \\
\text { States }\end{array}$ & $\begin{array}{c}\left(10^{9} \mathrm{kWh} /\right. \\
\text { year })\end{array}$ & $\begin{array}{c}\% \text { of } \\
\text { United } \\
\text { States }\end{array}$ & $\begin{array}{c}(\mathrm{MMT} / \\
\text { yearr) }\end{array}$ \\
\hline 20 & 10 & 30 & $\begin{array}{l}\text { Fuel Savings for } 0.1 \% \text { Additional } \\
\text { Regulation Requirement }\end{array}$ & 5 & 0.02 & 1 & 0.02 & 1 \\
\hline
\end{tabular}

- Regulation

- The quantity of energy reduction $(1 \mathrm{~B} \mathrm{kWh}))$ is calculated by applying the reduction $(20 \%)$ in energy consumption to the quantity of total quantity of peak load electricity supplied to the grid $(5 \mathrm{~B} \mathrm{kWh})$. This amount is calculated as the $0.1 \%$ need for additional regulation times the electricity supplied to the grid (4968 B kWh - AEO).

- The percentage reduction in electricity $(0.02 \%)$ is calculated by dividing the quantity reduced (1 $\mathrm{B} \mathrm{kWh}$ ) by the total quantity of electricity supplied to the grid (4968 B kWh - AEO).

- The $\mathrm{CO}_{2}$ emission reduction (0.6 MMT) is calculated by dividing the quantity electricity reduction $(1 \mathrm{~B} \mathrm{kWh})$ by the total electricity supplied to the grid (4968 B kWh - AEO) times total utility sector emissions (2948 MMT - AEO).

- The percentage of $\mathrm{CO}_{2}$ emission reduction $(0.02 \%)$ is calculated by dividing the reduction $(0.6$ MMT) by total utility emissions (2948 MMT -AEO).

\section{Assumptions}

- The need for additional regulation for an RPS of $20 \%$ is $0.1 \%$.

- Regulation energy savings are $20 \%$ of peak load from a range of $10 \%$ to $30 \%$.

- Penetration $=100 \%$

- The calculations apply to the integration of solar PVs, so wind and/or solar PVs can be used to meet the $20 \%$ RPS.

\section{$\underline{\text { Data Sources }}$}

- Requirement of $0.1 \%$ additional regulation Source: Smith JC, B Parsons, T Acker, M Milligan, R Zavadil, M Schuerger, and E DeMeo. 2007. "Best Practices in Grid Integration of Variable Wind Power: Summary of Recent U.S. Case Study Results and Mitigation Measures." In: European Wind Energy Conference 2007, May, Milan, Italy,.

- Electricity Supply in 2030 is $4968 \mathrm{~B} \mathrm{kWh}$

Source: AEO 2008 (DOE/EIA-0383), Table 8. Electricity Supply, Disposition, Prices, and Emissions; Total Net Generation to the Grid

- Electric Utility $\mathrm{CO}_{2}$ Emissions in 2030 is $2948 \mathrm{MMT}$

Source: AEO 2008 (DOE/EIA-0383), Table 18. Carbon Dioxide Emissions by Sector and Source; Electric Power Total 


\section{Indirect Savings Mechanism B Joint Marketing of Energy Efficiency and Demand Response Programs}

\begin{tabular}{|c|c|c|c|c|c|c|c|c|c|}
\hline \multirow[b]{3}{*}{$\begin{array}{c}\text { Est. } \\
\%\end{array}$} & \multicolumn{5}{|c|}{ Avoided Expenditure Reinvested to Save Carbon (2030) } & \multicolumn{4}{|c|}{ Electric Sector Annual Reductions (2030) } \\
\hline & \multirow[b]{2}{*}{$\begin{array}{c}\text { Low } \\
\%\end{array}$} & \multirow[b]{2}{*}{$\begin{array}{c}\text { High } \\
\%\end{array}$} & \multicolumn{2}{|l|}{ Baseline Captial Expenditure } & \multirow[b]{2}{*}{$\begin{array}{l}\text { Savings } \\
\left(10^{9} \$\right)\end{array}$} & \multicolumn{2}{|c|}{ Energy } & \multicolumn{2}{|c|}{ Carbon Emissions } \\
\hline & & & Investment & $\left(10^{9} \$\right)$ & & $\begin{array}{l}\% \text { of } \\
\text { United } \\
\text { States }\end{array}$ & $\begin{array}{c}\left(10^{9} \mathrm{kWh} /\right. \\
\text { year })\end{array}$ & $\begin{array}{l}\% \text { of } \\
\text { United } \\
\text { States }\end{array}$ & $\begin{array}{c}\text { (MMT/ } \\
\text { year) }\end{array}$ \\
\hline 0 & 0 & 1 & $\begin{array}{l}10 \% \text { Demand Response, Residential } \\
\text { @ } \$ 400 / \mathrm{kW} \& 8.8 \phi / \mathrm{kWh}\end{array}$ & 15 & 0.0 & & & & \\
\hline 0 & 0 & 1 & $\begin{array}{l}\text { 10\% Demand Response, } \\
\text { Small/Medium Commercial } \\
\text { Buildings @ } \$ 300 / \mathrm{kW} \& 8.8 \phi / \mathrm{kWh}\end{array}$ & 6 & 0.0 & 0 & 0 & 0 & 0 \\
\hline
\end{tabular}

- Residential Sector

- The baseline quantity of capital cost attributable to $10 \%$ demand response is calculated by prorating total generating capacity $(1111 \mathrm{GW}-\mathrm{AEO})$ by the share of residential electricity consumption ( $35 \%-\mathrm{AEO})$. This capacity $(385 \mathrm{GW})$ is then multiplied by the $10 \%$ that is affected by demand response, then multiplied by $\$ 400 / \mathrm{kW}$ to obtain the baseline value of $\$ 15 \mathrm{~B}$.

- Since the estimated electricity reductions from the synergy between energy efficiency and demand response programs is expected to be zero, there are no savings in capital investments that can be re-invested-therefore, there are no reductions in electricity and emissions attributable to this measure.

If, for example, capital expenditures decreased by $1 \%$, then the reduction in energy and $\mathrm{CO}_{2}$ emissions would be $2 \mathrm{~B} \mathrm{kWh}(0.04 \%)$ and $1 \mathrm{MMT}$ ton $(0.04 \%)$, respectively.

- Small/Medium Commercial Buildings

- The baseline quantity of capital cost attributable to the $10 \%$ demand response is calculated by prorating total generating capacity $(1111 \mathrm{GW}$ - AEO) that serves Small/Medium Commercial buildings by the share of 2030 commercial sector electricity consumption consumed by Small/Medium Commercial buildings (17\% - CBECS). This capacity (189 GW) is then multiplied by the $10 \%$ that is affected by demand response, and then multiplied by $\$ 300 / \mathrm{kW}$ to obtain the baseline value of $\$ 6 \mathrm{~B}$.

- Since the estimated electricity reductions from the synergy between energy efficiency and demand response programs is expected to be zero, there are no savings in capital investments that can be re-invested-therefore there are no reductions in energy and emissions attributable to this measure

If, for example, capital expenditures decreased by $1 \%$, then the reduction in energy and $\mathrm{CO}_{2}$ emissions would be $1 \mathrm{~B} \mathrm{kWh}(0.01 \%)$ and $0.4 \mathrm{MMT}$ ton $(0.01 \%)$, respectively.

Assumptions

- Sector savings of $0 \%$

- Penetration $=100 \%$ 
- Small/Medium Commercial buildings are defined as less than 50,000 $\mathrm{ft}^{2}$ in floor area.

- The 2030 Small/Medium Commercial building share of total commercial sector electricity consumption is the same as the 2003 share.

- The values of $\$ 400 / \mathrm{kW}$ and $\$ 300 / \mathrm{kW}$ are for the estimated installed cost of demand response control and communication equipment in the residential and commercial sectors, respectively.

\section{Data Sources}

- Residential Sector electricity consumption in 2030 is $1722 \mathrm{~B} \mathrm{kWh}$ Source: AEO 2008 (DOE/EIA-0383), Table 8. Electricity Supply, Disposition, Prices, and Emissions; Electricity Sales by Sector

- Commercial Sector electricity consumption in 2030 is $1941 \mathrm{~B} \mathrm{kWh}$ Source: AEO 2008 (DOE/EIA-0383), Table 8. Electricity Supply, Disposition, Prices, and Emissions; Electricity Sales by Sector

- Small/Medium Commercial building consumption as share of total commercial sector consumption in 2003 is $44 \%$

Source: 2003 CBECS Detailed Tables, Table E5A. Electricity Consumption (kWh) by End Use for All Buildings, 2003

- Electricity Supply in 2030 is $4968 \mathrm{~B}$ kWh

Source: AEO 2008 (DOE/EIA-0383), Table 8. Electricity Supply, Disposition, Prices, and Emissions; Total Net Generation to the Grid

- Electric Generating Capacity in 2030 is $1111 \mathrm{GW}$

Source: AEO 2008 (DOE/EIA-0383), Table 9. Electricity Generating Capacity gigawatts)

- Electric Utility $\mathrm{CO}_{2}$ Emissions in 2030 is 2948 MMT

Source: AEO 2008 (DOE/EIA-0383), Table 18. Carbon Dioxide Emissions by Sector and Source; Electric Power Total 


\section{Indirect Savings Mechanism D Measurement \& Verification for Energy Efficiency Programs}

\begin{tabular}{|c|c|c|c|c|c|c|c|c|c|}
\hline \multirow[b]{3}{*}{$\begin{array}{c}\text { Est. } \\
\%\end{array}$} & \multicolumn{4}{|c|}{ Avoided Expenditure Reinvested to Save Carbon (2030) } & & \multicolumn{4}{|c|}{ Electric Sector Annual Reductions (2030) } \\
\hline & \multirow[b]{2}{*}{$\begin{array}{l}\text { Low } \\
\%\end{array}$} & \multirow[b]{2}{*}{$\begin{array}{c}\text { High } \\
\%\end{array}$} & \multicolumn{2}{|l|}{ Baseline Captial Expenditure } & \multirow[b]{2}{*}{$\begin{array}{c}\text { Savings } \\
\left(10^{9} \$\right)\end{array}$} & \multicolumn{2}{|c|}{ Energy } & \multicolumn{2}{|c|}{ Carbon Emissions } \\
\hline & & & Investment & $\left(10^{9} \$\right)$ & & $\begin{array}{l}\text { \% of } \\
\text { United } \\
\text { States }\end{array}$ & $\begin{array}{l}\left(10^{9} \mathrm{kWh} /\right. \\
\text { year })\end{array}$ & $\begin{array}{l}\% \text { of } \\
\text { United } \\
\text { States }\end{array}$ & $\begin{array}{c}\text { (MMT/ } \\
\text { year) }\end{array}$ \\
\hline 1 & 0 & 2 & $\begin{array}{l}10 \% \text { Energy Efficiency, Residential } \\
\text { @ } 8.8 \notin / \mathrm{kWh}, 10 \text {-Year Life }\end{array}$ & 152 & 1.5 & & & & \\
\hline 1 & 0 & 2 & $\begin{array}{l}\text { 10\% Energy Efficiency, } \\
\text { Small/Medium Commercial } \\
\text { Buildings @ } 8.8 \notin / \mathrm{kWh}, 10 \text {-Year Life }\end{array}$ & 75 & 0.8 & 0.5 & 26 & 0.5 & 15 \\
\hline
\end{tabular}

- Residential Sector

- The baseline value ( $\$ 152 \mathrm{~B}$ ) is calculated as the quantity of energy (1702 B kWh - AEO) attributable to the $10 \%$ improvement in energy efficiency that would provide savings over a 10 year period (1702 B kWh) multiplied by the average price of electricity in 2030 (\$0.088).

- The reduction attributable to this value $(\$ 1.5 \mathrm{~B})$ is the expected $1 \%$ reduction.

- The corresponding amount of efficiency that can be purchased through reinvesting the $\$ 1.5 \mathrm{~B}$ is calculated by dividing this amount by the cost of electricity $(\$ 0.088)$ to provide $17 \mathrm{~B} \mathrm{kWh}$.

- The percentage reduction in electricity consumption $(0.35 \%)$ is the reduction $(17 \mathrm{~B} \mathrm{kWh})$ divided by total electricity supply (4968 B kWh - AEO).

- The $\mathrm{CO}_{2}$ emission reduction (10 MMT) is calculated by dividing the electricity reduction (26 B $\mathrm{kWh}$ ) by the total electricity supplied to the grid (4968 B kWh - AEO) times total utility sector emissions (2948 MMT - AEO).

- The percentage of $\mathrm{CO}_{2}$ emission reduction $(0.35 \%)$ is calculated by dividing the reduction (10 MMT) by total utility emissions (2948 MMT -AEO).

- Small/Medium Commercial Buildings

- The baseline value ( $\$ 75 \mathrm{~B}$ ) is calculated as the quantity of energy (854 B kWh) attributable to the $10 \%$ improvement in energy efficiency that would provide savings over a 10-year period (854 B $\mathrm{kWh}$ ) multiplied by the average price of electricity in 2030 (\$0.088). Small/Medium Commercial building electricity consumption ( $854 \mathrm{~B} \mathrm{kWh}$ ) is calculated by multiplying the 2003 Small/Medium Commercial building share of electricity (44\% - CBECS) by commercial sector consumption (1941 B kWh - AEO).

- $\quad$ The reduction attributable to this value $(\$ 0.8 \mathrm{~B})$ is the expected $1 \%$ reduction.

- The corresponding amount of efficiency that can be purchased through reinvesting the $\$ 0.8 \mathrm{~B}$ is calculated by dividing this amount by the cost of electricity $(\$ 0.088)$ to provide $9 \mathrm{~B} \mathrm{kWh}$.

- The percentage reduction in electricity consumption $(0.17 \%)$ is the reduction $(9 \mathrm{~B} \mathrm{kWh})$ divided by total electricity supply (4968 B kWh - AEO).

- The $\mathrm{CO}_{2}$ emission reduction (5 MMT) is calculated by dividing the electricity reduction (8 $\mathrm{B}$ $\mathrm{kWh}$ ) by the total electricity supplied to the grid (4968 B kWh - AEO) times total utility sector emissions (2948 MMT - AEO). 
- The percentage of $\mathrm{CO}_{2}$ emission reduction $(0.17 \%)$ is calculated by dividing the reduction (5 MMT) by total utility emissions (2948 MMT -AEO).

\section{$\underline{\text { Assumptions }}$}

- Efficiency achieved by 2030 is $10 \%$

- 10-year average payback

- Sector savings of $1 \%$

- Penetration $=100 \%$

- Small/Medium Commercial buildings are defined as less than 50,000 $\mathrm{ft}^{2}$ in floor area.

- The 2030 Small/Medium Commercial building share of total commercial sector electricity consumption is the same as the 2003 share (44\%).

- The levelized installed average cost of efficiency measures equals the average cost of electricity of $\$ .088 / \mathrm{kWh}$.

\section{Data Sources}

- Residential Sector electricity consumption in 2030 is $1722 \mathrm{~B} \mathrm{kWh}$ Source: AEO 2008 (DOE/EIA-0383), Table 8. Electricity Supply, Disposition, Prices, and Emissions; Electricity Sales by Sector

- Commercial Sector electricity consumption in 2030 is $1941 \mathrm{~B} \mathrm{kWh}$ Source: AEO 2008 (DOE/EIA-0383), Table 8. Electricity Supply, Disposition, Prices, and Emissions; Electricity Sales by Sector

- Small/Medium Commercial building consumption as share of total commercial sector consumption in 2003 is $44 \%$

Source: 2003 CBECS Detailed Tables, Table E5A. Electricity Consumption (kWh) by End Use for All Buildings, 2003

- Electricity Supply in 2030 is $4968 \mathrm{~B} \mathrm{kWh}$

Source: AEO 2008 (DOE/EIA-0383), Table 8. Electricity Supply, Disposition, Prices, and Emissions; Total Net Generation to the Grid

- Electric Utility $\mathrm{CO}_{2}$ Emissions in 2030 is $2948 \mathrm{MMT}$

Source: AEO 2008 (DOE/EIA-0383), Table 18. Carbon Dioxide Emissions by Sector and Source; Electric Power Total 


\section{Indirect Savings Mechanism I \\ Support Penetration of Renewable Wind Generation (20\% RPS)}

\begin{tabular}{|c|c|c|c|c|c|c|c|c|c|}
\hline \multirow[b]{3}{*}{$\begin{array}{c}\text { Est. } \\
\%\end{array}$} & \multicolumn{4}{|c|}{ Avoided Expenditure Reinvested to Save Carbon (2030) } & & \multicolumn{4}{|c|}{ Electric Sector Annual Reductions (2030) } \\
\hline & \multirow[b]{2}{*}{$\begin{array}{c}\text { Low } \\
\%\end{array}$} & \multirow[b]{2}{*}{$\begin{array}{l}\text { High } \\
\%\end{array}$} & \multicolumn{2}{|l|}{ Baseline Captial Expenditure } & \multirow[b]{2}{*}{$\begin{array}{l}\text { Savings } \\
\left(10^{9} \$\right)\end{array}$} & \multicolumn{2}{|c|}{ Energy } & \multicolumn{2}{|c|}{ Carbon Emissions } \\
\hline & & & Investment & $\left(10^{9} \$\right)$ & & $\begin{array}{l}\% \text { of } \\
\text { United } \\
\text { States }\end{array}$ & $\begin{array}{c}\left(10^{9} \mathrm{kWh} /\right. \\
\text { year })\end{array}$ & $\begin{array}{l}\% \text { of } \\
\text { United } \\
\text { States }\end{array}$ & $\begin{array}{l}\text { (MMT/ } \\
\text { year) }\end{array}$ \\
\hline 2 & 1 & 3 & $\begin{array}{l}1111 \mathrm{GW} \text { Total Generation Capacity } \\
\text { @ } \$ 1000 / \mathrm{kW}\end{array}$ & 1111 & 22 & 5 & 253 & 5 & 150 \\
\hline
\end{tabular}

- Ancillary Services

- The baseline avoided cost of the added generating capacity $(\$ 1111 \mathrm{~B})$ needed to supply adequate reserves for the $20 \%$ RPS is calculated as the product of the capacity $(1111 \mathrm{GW}-\mathrm{AEO}$ at $\$ 1000 / \mathrm{kW}$.

- The reduction attributable to this value (\$22 B) is the expected $2 \%$ reduction (range of $1-3 \%$ ) times the value of the generating capacity.

- The corresponding amount of efficiency $(253 \mathrm{kWh})$ that can be obtained by reinvesting the \$22 B reduction is the reduction divided by the average cost of electricity $(\$ 0.088)$.

- This amount is then divided by total electricity supply (4968 B kWh) to obtain the percentage reduction $(5 \%)$.

- The $\mathrm{CO}_{2}$ emission reduction (150 MMT) is calculated by dividing the electricity reduction (253 B $\mathrm{kWh}$ ) by the total electricity supplied to the grid (4968 B kWh - AEO) times total utility sector emissions (2948 MMT - AEO).

- The percentage of $\mathrm{CO}_{2}$ emission reduction (5\%) is calculated by dividing the reduction (150 MMT) by total utility emissions (2948 MMT -AEO).

\section{Assumptions}

- Sector savings of $2 \%$ from eliminated need for increased operating reserve from a range of $5 \%$ to $7 \%$ peak load capacity.

- Penetration $=100 \%$

- The average price of generation capacity to provide ancillary services is $\$ 1000 / \mathrm{kW}$.

- The levelized cost of efficiency measures equals the average cost of electricity.

- The calculations apply to the integration of solar PVs, so wind and/or solar PVs can be used to meet the $20 \%$ RPS.

\section{$\underline{\text { Data Sources }}$}

- Electric Generating Capacity in 2030 is $1111 \mathrm{GW}$

Source: AEO 2008 (DOE/EIA-0383), Table 9. Electricity Generating Capacity gigawatts)

- Electricity Supply in 2030 is 4968 B kWh

Source: AEO 2008 (DOE/EIA-0383), Table 8. Electricity Supply, Disposition, Prices, and Emissions; Total Net Generation to the Grid 
- Electric Utility $\mathrm{CO}_{2}$ Emissions in 2030 is $2948 \mathrm{MMT}$

Source: AEO 2008 (DOE/EIA-0383), Table 18. Carbon Dioxide Emissions by Sector and Source; Electric Power Total

- Additional operating reserve requirement of $2 \%$

Source: Smith JC, B Parsons, T Acker, M Milligan, R Zavadil, M Schuerger, and E DeMeo. 2007.

"Best Practices in Grid Integration of Variable Wind Power: Summary of Recent U.S. Case Study

Results and Mitigation Measures." In: European Wind Energy Conference 2007, May, Milan, Italy. 


\section{Attachment 2 References for Data Sources}

DOE/EIA - U.S. Department of Energy/U.S. Energy Information Administration. 2003. Table A6. Building Size, Floorspace for All Buildings (Including Malls). Accessed December 29, 2009, at http://www.eia.doe.gov/emeu/cbecs/cbecs2003/detailed_tables_2003/detailed_tables_2003.html\#end use03.

DOE/EIA - U.S. Department of Energy/U.S. Energy Information Administration. 2003. Table E5A. Electricity Consumption (kWh) by End Use for All Buildings. Accessed December 29, 2009, at http://www.eia.doe.gov/emeu/cbecs/cbecs2003/detailed tables_2003/detailed_tables_2003.html\#end use03.

DOE/EIA - U.S. Department of Energy/U.S. Energy Information Administration. 2005. Table HC2.4 Space Heating Characteristics by Type of Housing Unit, 2005. Accessed December 29, 2009, at http://www.eia.doe.gov/emeu/cbecs/cbecs2003/detailed tables_2003/detailed tables_2003.html\#end use03.

DOE/EIA - U.S. Department of Energy/U.S. Energy Information Administration. 2008. Annual Energy Outlook 2008. DOE/EIA-0383(2008), U.S. Department of Energy, Washington, D.C. Accessed December 29, 2009, at http://www.eia.doe.gov/oiaf/archive/aeo08/index.html.

Smith JC, B Parsons, T Acker, M Milligan, R Zavadil, M Schuerger, and E DeMeo. 2007. "Best Practices in Grid Integration of Variable Wind Power: Summary of Recent U.S. Case Study Results and Mitigation Measures.” In: European Wind Energy Conference 2007, May, Milan, Italy. 



\title{
Distribution
}

No. of

Copies

16 PDF Copies

S Hauser

L Coogan

K Hamilton

N Sanai

J Paladino

R Rusack

M Oldak

$\mathrm{J}$ Berst

J Mandel

M McGranahan

D Von Dollen

P Centolella

K Corum

J Wellinghoff

L Schwartz

C McDermott
No. of

Copies

\author{
steve.hauser@nrel.gov \\ lcoogan@navista.net \\ khamilton@gridwise.org \\ Nick.Sinai@fcc.gov \\ joseph.paladino@netl.doe.gov \\ Rick.Rusack@litosad.com \\ Mike.Oldak@UTC.org \\ jesse.berst@globalsmartenergy.com \\ jmandel@eenews.net \\ mmmcgranahan@epri.com \\ dvondollen@epri.com \\ Paul.Centolella@puc.state.oh.us \\ kcorum@nwcouncil.org \\ Jon.Wellinghoff@ferc.gov \\ LSchwartz@raponline.org \\ cmcdermott@rockportcap.com
}

Distr.1 




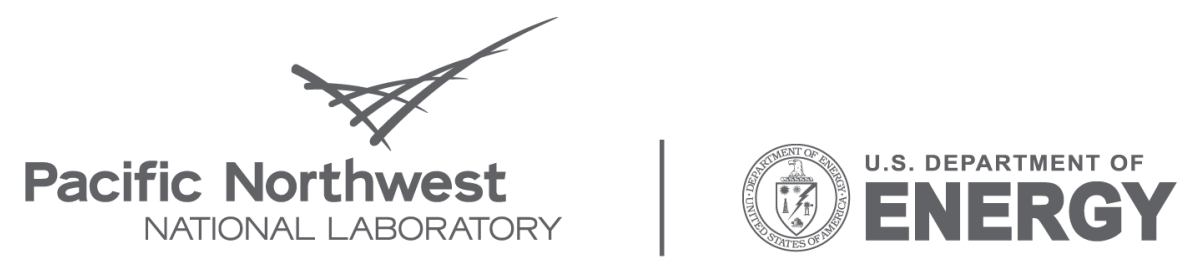

Proudly Operated by Battelle Since 1965

902 Battelle Boulevard

P.O. Box 999

Richland, WA 99352

1-888-375-PNNL (7665)

www.pnl.gov 